\title{
BRIDGE DESIGN FOR EARTHQUAKE FAULT CROSSINGS: SYNTHESIS OF DESIGN ISSUES AND STRATEGIES
}

\author{
A Thesis \\ Presented to the Faculty of \\ California Polytechnic State University \\ San Luis Obispo
}

In Partial Fulfillment

of the Requirements for the Degree of

Master of Science in Civil \& Environmental Engineering

By

Osmar Rodriguez

March, 2012 
(C) 2012

Osmar Rodriguez

\section{ALL RIGHTS RESERVED}




\section{COMMITTEE MEMBERSHIP}

TITLE:

Bridge Design for Earthquake Fault Crossings: Synthesis of Design Issues and Strategies

AUTHOR: $\quad$ Osmar Rodriguez

DATE SUBMITTED:

March, 2012

COMMITEE CHAIR: $\quad$ Dr. Rakesh Goel, Civil \& Environmental Engineering

Department Chair

COMMITTEE MEMBER: $\quad$ Dr. Bing Qu, Civil \& Environmental Engineering

Assistant Professor

COMMITTEE MEMBER: $\quad$ Dr. Eric Kasper, Civil \& Environmental Engineering

Assistant Professor 


\section{ABSTRACT}

Bridge Design for Earthquake Fault Crossings: Synthesis of Design Issues and Strategies

\section{By Osmar Rodriguez}

This research evaluates the seismic demands for a three-span curved bridge crossing fault rupture zones. Two approximate procedures which have been proved adequate for ordinary straight bridges crossing fault-rupture zones, i.e., the fault-rupture response spectrum analysis (FR-RSA) procedure and the fault-rupture linear static analysis (FR-LSA) procedure, were considered in this investigation. These two procedures estimate the seismic demands by superposing the peak values of quasi-static and dynamic bridge responses. The peak quasi-static response in both methods is computed by nonlinear static analysis of the bridge under the ground displacement offset associated with fault-rupture. In FR-RSA and FR-LSA, the peak dynamic responses are respectively estimated from combination of the peak modal responses using the completequadratic-combination rule and the linear static analysis of the bridge under appropriate equivalent seismic forces. The results from the two approximate procedures were compared to those obtained from the nonlinear response history analysis (RHA) which is more rigorous but may be too onerous for seismic demand evaluation. It is shown that the FR-RSA and FR-LSA procedures which require less modeling and analysis efforts provide reasonable seismic demand estimates for practical applications. 


\section{ACKNOWLEDGMENTS}

It is a pleasure to thank Dr. Rakesh Goel for his support, supervision, and wisdom throughout this entire process. Thanks to him and his acceptance onto this project, I have found my strengths in seismic and structural engineering. I am thoroughly honored to have worked under such direction and greatly respect your accomplishments and advancements in these fields.

I offer my sincerest gratitude to my assistant thesis advisor, Dr. Bing Qu, who has supported me throughout this project with his knowledge, patience, and guidance. I attribute the level of my master's degree to his encouragement and effort and without him this thesis, too, would not have been possible, thank you. To Dr. Eric Kasper for taking time out of his schedule to take part in my thesis review committee and providing important advice on how to refine certain aspects of my research. Also to all of my professors at Cal Poly who have inspired and guided me through my college career.

To my colleagues Tòmas Bowdey, Colin Leung, Jacky Ng, and Rachel Goosens for holding the utmost self-discipline while going through this program together. I look forward to seeing what each of your careers and future bring. To my close friends who have been with me from day one, I appreciate your patience and forbearance through all this; I could not have done it without all of you teasing and pushing me daily to finish already.

To my girlfriend Alyssa who has been with me far before I became an engineering master, thank you for everything you do and the person you are. Your ability to pretend to care about or listen to me talk about any of these technical concepts without leaving me amazes me. I am fortunate to have found someone as beautiful and caring as you.

To God, thank you for giving me strength to continue moving forward every day. Lastly, to my mother and father for encouraging my sister and me to become anything we wanted and supporting us whole-heartedly through our path there. For showing us what true hard work is and how anything is obtainable through it, thank you. This thesis is dedicated to you. 
The research reported in this thesis is supported by CALTRANS under Contract No. 65A0379 with Dr. Allaoua Kartoum as the project manager. The support is gratefully acknowledged. We acknowledge Drs. Tom Shantz and Brian Chiou from the Division of Research and Innovation of CALTRANS who simulated the ground motions used in this investigation. We are also grateful to Mark Yashinsky, Foued Zayati, Mark Mahan, and Toorak Zokaie of CALTRANS for their useful feedback on this research investigation. Moreover, the original finite element bridge model was developed by Dr. Farzin Zareian from University of California at Irvine. 


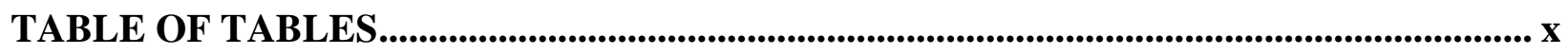

TABLE OF FIGURES.................................................................................................................... xi

CHAPTER 1: STATEMENT OF RESEARCH............................................................................ 1

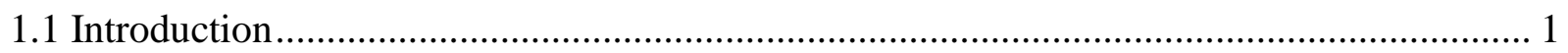

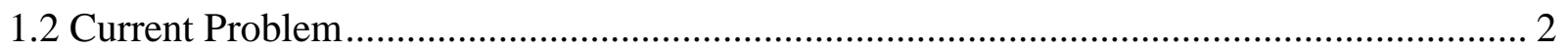

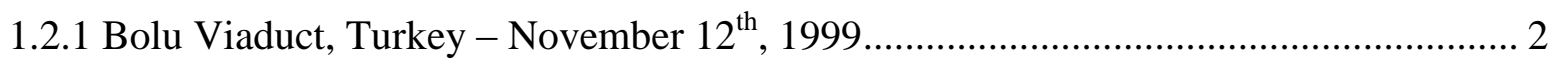

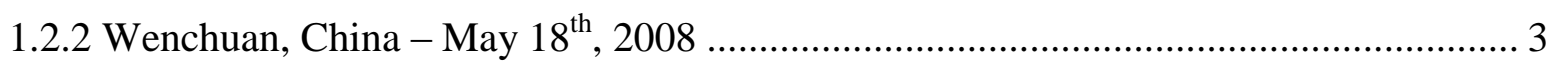

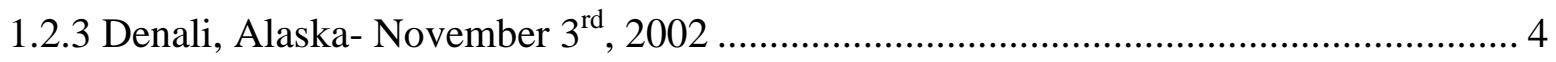

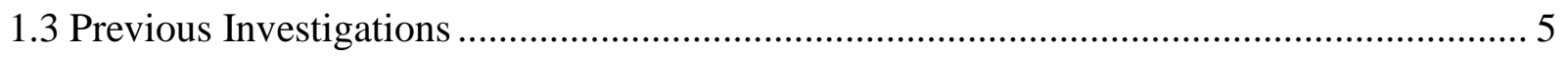

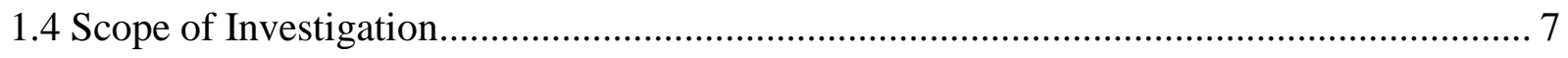

1.4.1 Phase 1: Identification of Bridge Examples.............................................................. 7

1.4.2 Phase 2: Selection of Ground Motion Histories and Design Spectrum .......................... 9

1.4.3 Phase 3: Development of Computer Models ……………....................................... 10

1.4.4 Phase 4: Evaluation of Previous Procedures............................................................... 10

1.4.5 Phase 5: Evaluation of Analysis Procedures for Practical Use..................................... 11

CHAPTER 2: REVIEW OF EXISTING ANALYSIS METHODS ....................................... 12

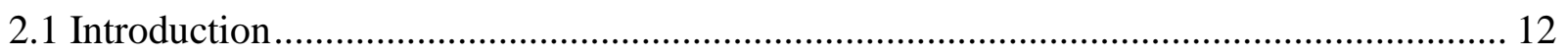

2.2 Existing Analysis Methods for Bridges Crossing Fault-Rupture Zones............................. 12 
2.2.1 Theoretical Background: Linear Analysis 13

2.2.2 Nonlinear Response History Analysis ............................................................ 15

2.2.3 Fault-Rupture Response Spectrum Analysis ........................................................ 16

2.2.4 Fault-Rupture Linear Static Analysis............................................................... 18

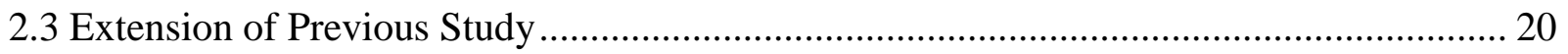

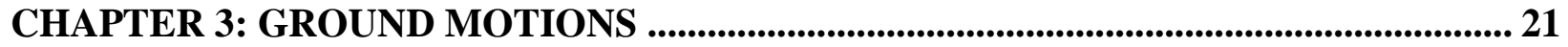

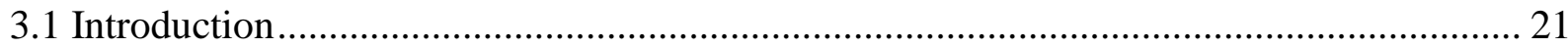

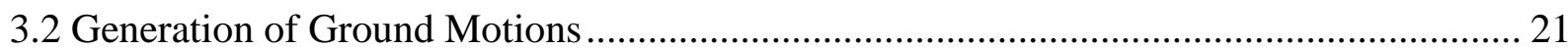

3.2.1 Selected Record Sets ............................................................................... 22

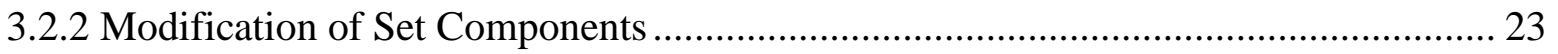

3.3 Ground Motion Analysis............................................................................................ 26

3.3.1 Spatially-Uniform Ground Motions............................................................. 27

3.3.2 Spatially-Varying Ground Motion Across Fault-Rupture Zones............................. 28

3.4 Development of CALTRANS Target Spectrum ................................................... 28

CHAPTER 4: DEVELOPMENT OF COMPUTER MODEL ........................................... 31

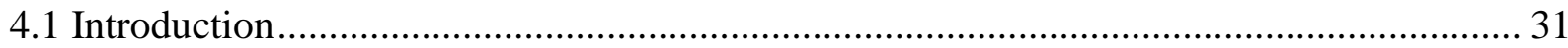

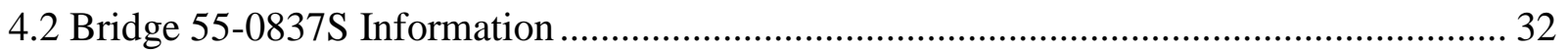

4.3 Linear and Nonlinear Models of Bridge 55-0837S................................................. 35

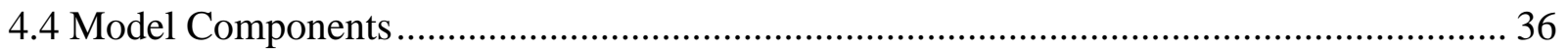




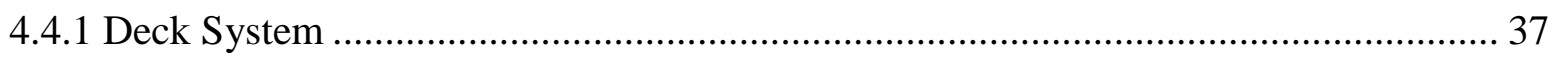

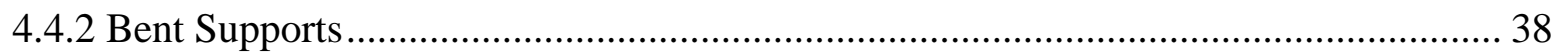

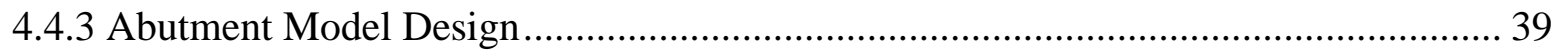

4.4.6 Zero-Length Members ................................................................................ 42

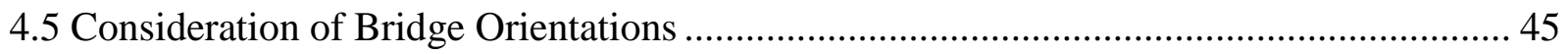

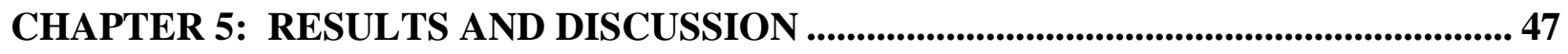

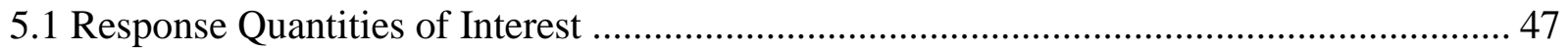

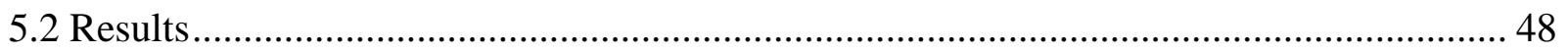

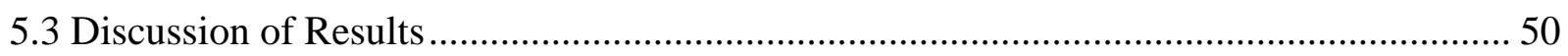

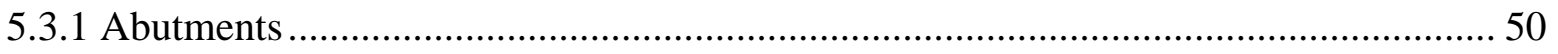

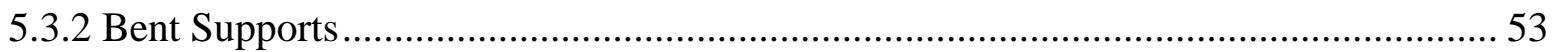

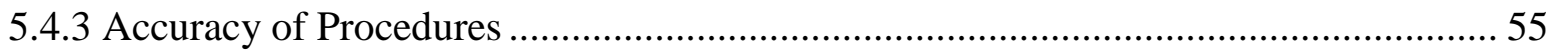

CHAPTER 6: CONCLUSIONS \& RECOMMENDATIONS .......................................... 57

CHAPTER 7: RECOMMENDATIONS FOR SUBSEQUENT STUDY ............................ 62

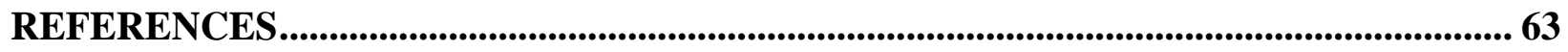

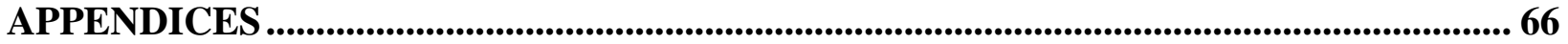

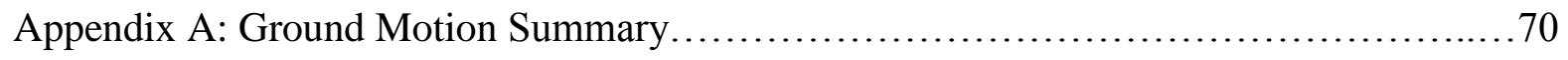

Appendix B: Mode Shapes and Periods.......................................92

Appendix C: Complete Result Comparisons of Bridge 55-0837S ....................111 


\section{TABLE OF TABLES}

Table 1. Summary of considered base ground motion pairs .............................................. 22

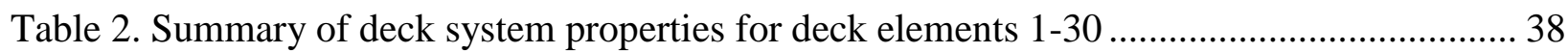

Table 3. Finite Element Model Zero-length member summary …..................................... 44 


\section{TABLE OF FIGURES}

Figure 1. Damage at western abutment of Bolu Viaduct, deck displacement due to support

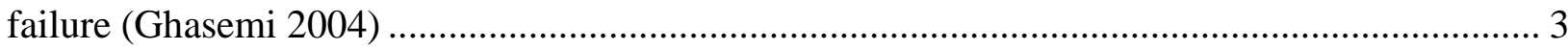

Figure 2. Full bridge collapse due to Wenchuan earthquake (Kawashima et al. 2008) ................ 4

Figure 3. Seismic support system along oil pipeline (Honegger et al. 2004) .............................. 5

Figure 4. Bridge 55-0837S (a) plan view and (b) elevation view ......................................... 9

Figure 5. Sketch of the effective influence vector for a bridge crossing fault rupture zones ....... 19

Figure 6. Example acceleration pulse that was added to Record Set 1 to create a $50 \mathrm{~cm}$ fling

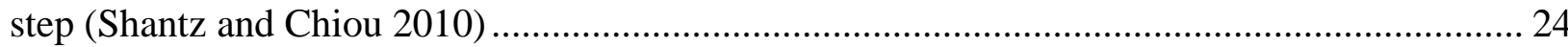

Figure 7. Example acceleration pulse that was added to Record Set 1 to create a $50 \mathrm{~cm}$ fling step

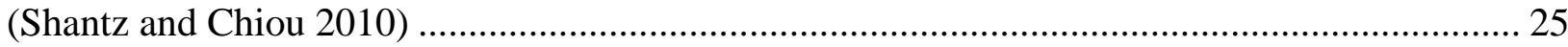

Figure 8. Time histories for (a) Rec1_BN90 and (b) Rec1_BP90 .......................................... 26

Figure 9. CALTRANS design spectrum plotted against 10 record sets (a) and against the

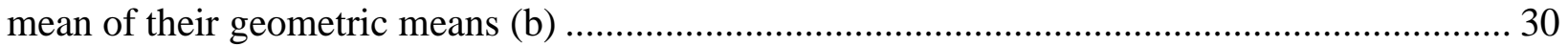

Figure 10. Plan and elevation views of Bridge 55-0837S ................................................. 32

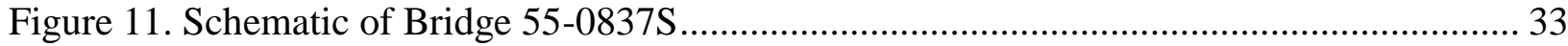

Figure 12. Single box girder typical section for Bridge 55-0837S ........................................ 34

Figure 13. Bent support cross-sections for Bridge 55-0837S ............................................ 34

Figure 14. Bent support foundation typical section for Bridge 55-0837S ............................. 35

Figure 15. Assignments of nodes and elements in plan view for the FE model of Bridge 55$0837 \mathrm{~S}$ 
Figure 16. Assignments of nodes and elements in elevation view for the FE model of Bridge 55-0837S 39

Figure 17. Simplification of longitudinal abutment springs 41

Figure 18. Actual bent support cross-section (a) and expected inelastic regions at bent support (b)

Figure 19. Location of zero-length member to a beam-column element................................ 44

Figure 20. Definition of bridge orientation angle ........................................................ 46

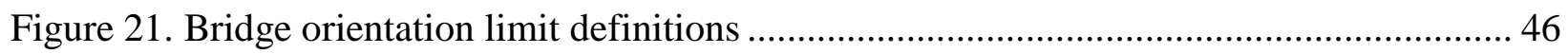

Figure 22. Results for $\theta=0$ degrees and longitudinal abutment stiffness $=0.10 \mathrm{~K}_{\text {eff } \ldots \ldots \ldots \ldots \ldots . .} 49$

Figure 23. Results for $\theta=0$ degrees and longitudinal abutment stiffness $=0.55 \mathrm{~K}_{\mathrm{eff}} \ldots \ldots \ldots \ldots \ldots . . .49$

Figure 24. Results for $\theta=0$ degrees and longitudinal abutment stiffness $=1.00 \mathrm{~K}_{\text {eff } \ldots \ldots \ldots \ldots \ldots \ldots . . . . . .50}$

Figure 25. Accuracy of FR-RSA for abutments in transverse direction at all orientations using

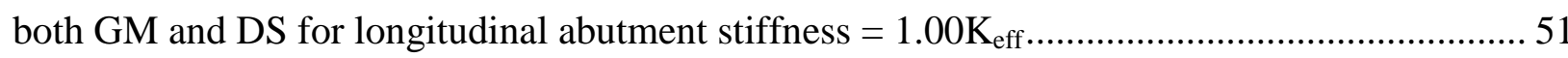

Figure 26. Accuracy of FR-RSA for abutments in longitudinal directions at all orientations using both GM and DS for longitudinal abutment stiffness $=1.00 \mathrm{~K}_{\mathrm{eff}}$ 52

Figure 27. Accuracy of FR-LSA for abutments in transverse directions at all orientations using both GM and DS for longitudinal abutment stiffness $=1.00 \mathrm{~K}_{\mathrm{eff}}$ 52

Figure 28. Accuracy of FR-LSA for abutments in longitudinal directions at all orientations using both GM and DS for longitudinal abutment stiffness $=1.00 \mathrm{~K}_{\mathrm{eff}}$ 53

Figure 29. Accuracy of FR-RSA for bents at all orientations using both GM and DS for longitudinal abutment stiffness $=1.00 \mathrm{~K}_{\mathrm{eff}}$ 54 
Figure 30. Accuracy of FR-LSA for bents at all orientations using both GM and DS for

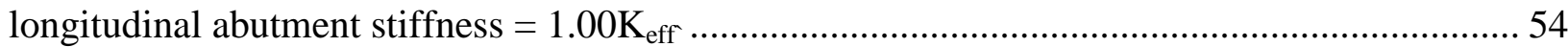




\section{CHAPTER 1: STATEMENT OF RESEARCH}

\subsection{Introduction}

In response to the damage recently sustained by California bridges during recent earthquakes, the California Department of Transportation (Caltrans) has taken extensive measures to update seismic design procedures to more efficiently analyze bridges crossing fault lines. Currently there are over 13,000 bridges in California, including roughly 120 of them crossing fault-rupture zones or lying in very close proximity to them, therefore developing a more efficient and convenient procedure to analyze such cases has become crucial. Currently the most arduous yet most accurate method of analyzing seismic demands of bridges requires nonlinear response history analysis (RHA) in which dynamic support motions from spatially varying ground motions due to surface rupture are taken into account.

The investigation proposes to synthesize Caltrans's current design issues in procedures analyzing bridge structures near fault-rupture zones and provides measurements to overcome such issues. This thesis focuses on evaluation of the adequacy of two simplified analysis procedures developed and validated from by Goel and Chopra (Goel and Chopra 2008a, 2008b, 2009a, 2009b) i.e., (1) Fault-Rupture Linear Static Analysis (FR-LSA), and (2) Fault-Rupture Response Spectrum Analysis (FR-RSA) methods in estimating the seismic demands of a threespan curved bridge crossing a fault-rupture zone. Results from the simplified procedures were compared to the "exact" seismic demands produced from nonlinear RHA. By proving that new, more simplified methods of analysis are able to provide accurate displacement estimates, current 
design and analysis measures are able to be updated and provide a faster approach for bridge engineers to use.

The two presented procedures estimate the seismic demands on a bridge crossing earthquake fault by superposing the peak values of quasi-static and dynamic bridge responses. The peak quasi-static response in both methods is computed by nonlinear static analysis of the bridge under the ground displacement offset associated with fault rupture. In FR-RSA and FR-LSA, the peak dynamic responses are, respectively, estimated from combination of the peak modal responses using the complete-quadratic-combination (CQC) rule and linear static analysis of the bridge under appropriate equivalent seismic forces.

\subsection{Current Problem}

Although avoiding building bridges across fault lines all-together might be the best approach, such situations are not always permissible. Regions of high seismicity such as in California leave engineers without many options in design but instead create a great need for sound design over such cases. Recent earthquakes have shown the prodigious vulnerability of bridges crossing fault-rupture zones leaving them either damaged or in some cases fully collapsed. The following sections briefly review the damages of such bridges observed from previous earthquakes.

\subsubsection{Bolu Viaduct, Turkey - November $12^{\text {th }}, 1999$}

For example, spans of the newly-constructed Bolu viaduct along the trans-European expressway in Turkey were inches away from collapse in 1999 due to the fault movement along the transverse direction of the bridge. According to seismologists, the rupture on November 12, 1999 was caused by a strike-slip rupture along the secondary Duzce fault with a magnitude of 
7.2 with an epicenter located very close to the Bolu viaduct (Ghasemi 2004). The viaduct design included a hybrid isolation system which suffered complete failure and narrowly avoided total collapse due to excessive superstructure movement. The surface fault-rupture produced major propagation between segments of the viaducts piers and decks, as shown in Figure 1. The response of the Bolu Viaduct highlighted the importance of designing for high ground movement, especially for structures near active fault zones.

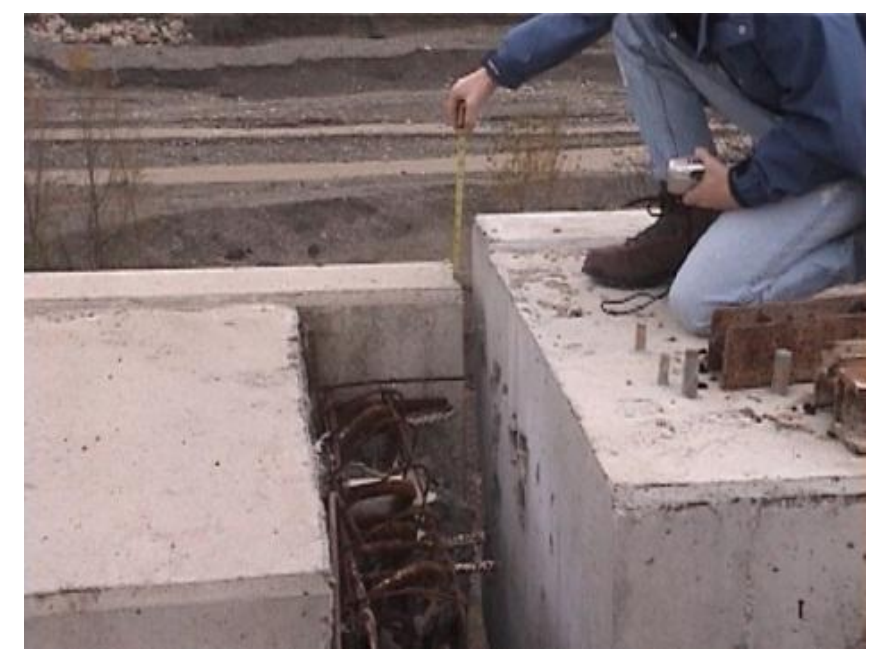

Figure 1. Damage at western abutment of Bolu Viaduct, deck displacement due to support failure (Ghasemi 2004)

\subsubsection{Wenchuan, China - May 18 ${ }^{\text {th }}, 2008$}

Recent earthquakes in Wenchuan, China severely damaged bridges located near active fault zones. While damage to bridges varied, several cases of full collapse were reported for bridges on top of the actual fault, as shown in Figure 2. The significant ground offsets caused extensive movement along the transverse direction of most of the bridges, leading to detachment of lateral beams from columns at the joints. It was noted that major dislocation of the bridges members 
occurred for curved bridges due to insufficient seismic design force and lack of ductility capacity (Kawashima et al. 2008). Other major damages to bridges included; absence of unseating prevention devices, bridge location crossing fault displacements, slope failures, and unreinforced stone masonry bridges. The damage done along main roads resulted in navigation obstacles for rescue teams and local rescue teams while damage done to local ordinary bridges produced heavy complications for evacuation of local citizens.

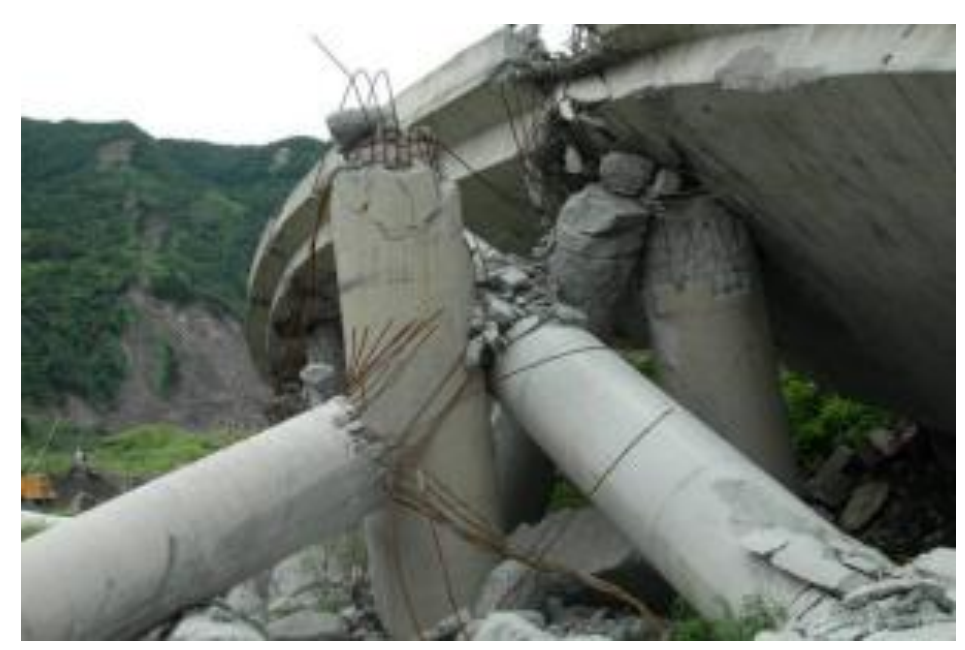

Figure 2. Full bridge collapse due to Wenchuan earthquake (Kawashima et al. 2008)

\subsubsection{Denali, Alaska- November $3^{\text {rd }}, 2002$}

It has been shown that sound design of bridge structures across active fault lines is not only possible but critical as well, as evident in the most recent earthquake occurring near the Denali fault in Alaska in which key segments of the trans-Alaska oil pipeline survived from a rightlateral strike-slip event. The lines held up against strike-lateral fault displacements caused by a 7.9 magnitude earthquake without any failures or leakage due to having proper seismic damage prevention systems as shown in Figure 3 (Honegger et al. 2004). Aside from the main shock, the 
Alaska Earthquake Information Center (AEIC) located over 35,000 aftershocks through the end of 2004 of which the structure withstood without serious damage. The near-source motions coupled with soil liquefaction developed significant bending and axial strain in the pipeline. The structure resisted the ground motions in a manner consistent with the original design premise, which allowed limited damage to the pipeline, support system (Nyman et al. 2006).

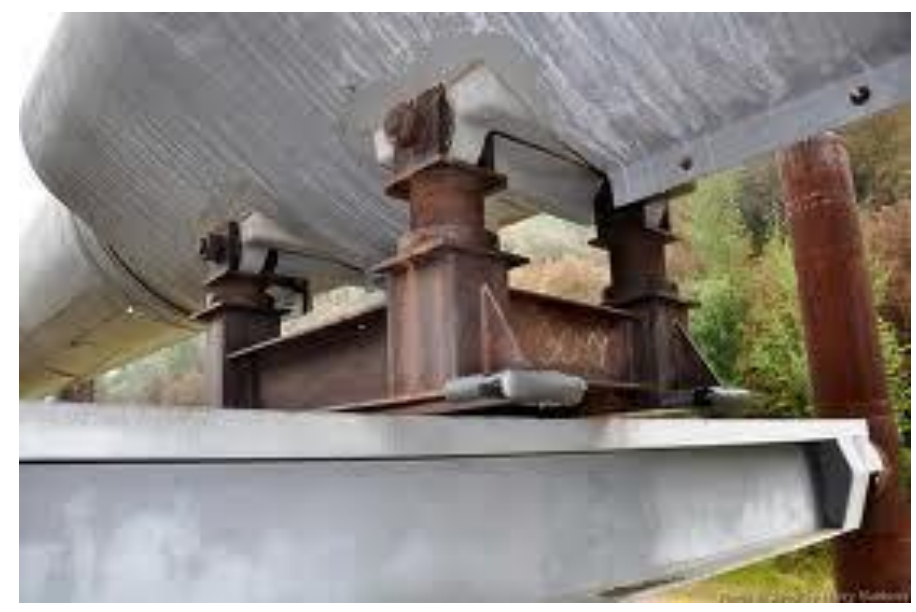

Figure 3. Seismic support system along oil pipeline (Honegger et al. 2004)

\subsection{Previous Investigations}

Classifications of bridges in California include both "lifeline" routes and "ordinary" bridges, whose designs are governed by CALTRANS Seismic Design Criteria (SDC)(CALTRANS 2010). While site-specific seismological studies to define spatially varying ground motions and rigorous nonlinear response history analysis (RHA) are necessary for important bridges on "lifeline" routes, such investigations may be too onerous for "ordinary" bridges. Seeing how a large amount of California's bridges are classified as "ordinary", the investigation proposes the 
validity behind simplified procedures to fill the need for providing accurate estimation of seismic demands and facilitate evaluation and design of such "ordinary" bridge scenarios.

In a previous investigation, Goel and Chopra (2008a) developed simplified procedures to analyze and compare seismic performance of bridges subjected to fault offset displacement and ground shaking. However, due to limited scope of the investigation, applicability of these procedures was demonstrated for idealized straight ordinary bridge models. Based on Goel and Chopra (2008a), this investigation extends the methods of approximation to actual curved bridges.

A comprehensive study by Afshin and Amjadian (2010) illustrated that bridges with complex geometries such as curved bridges are more susceptible to seismic damage than straight highway bridges with regular geometry. The study showed that coupling of translational and rotational movements of the deck effectively produce striking effects on the concrete catalyzing for possible deck or support rupture. Afshin and Amjadian (2010) modeled the deck of the superstructure as being dynamic in-plane, however with material properties remaining intact and linear during earthquake excitation, demonstrating normal behaviors unless under an irregularly large quake. The reinforced-concrete bent supports were modeled with rigid constraints and nodal mass elements that include the rotary mass of the support in three orthogonal directions (Ingham et al. 1997).

Tseng and Penzien (1975) produced a study of the coupled inelastic flexural behavior of bent supports by using a three-dimensional elastoplastic model, and the discontinuous behavior commonly seen in expansion joints by a nonlinear model used in this study (Chen 2001). In their 
model, important nonlinear characteristics such as effects of separation, impact, and slippage of soil-abutment interfaces were all implemented and accounted for. Since then, contributions to the development of nonlinear models for bridges have advanced greatly. Toki (1980) contributed to the implementation of nonlinear responses of continuous bridges subjected to traveling seismic waves, which is fully used in this investigation. Ghusn and Saiidi (1986) used bilinear biaxial bending elements (a five-spring system) for the interaction between pier columns and soil. Imbsen and Penzien (1986) considered the evaluation of energy-absorption characteristics of highway bridges including kinematic hardening features through the design of nonlinear beamcolumn elements for pier columns.

\subsection{Scope of Investigation}

In order to complete the work in a timely and logical manner, the research was broken down into the following phases:

\subsubsection{Phase 1: Identification of Bridge Examples}

A total of six existing bridge models crossing fault-rupture zones have been provided by Caltrans to for research. These bridges have been analyzed by researchers from the University of California, Irvine for other research purposes. The bridges included:

(1) Bridge 29-0315K - Jacktone SB99 On-Ramp Separation

(2) Bridge 29-0318 - Jacktone Road OH

(3) Bridge 53-2883S - Carson St - N605 Ramp

(4) Bridge 55-0837S - West Street - N5 On-Ramp

(5) Bridge 55-0938 - La Veta Ave. OC 
All bridges have been reviewed to be suitable to fit parameters needed for evaluation, including being three or four spans, with single or multi-column bents, and being skewed or curved. After detailed comparisons of all six bridges, Bridge 55-0837S (4) which is a three-span curved bridge built in 2000 located in east Anaheim, California (District 12 - Orange County), and serves as the West Street to Northbound 5 Interstate on-ramp was chosen to serve as the principal structure for investigation. The West Street Bridge serves greatly as a main on-ramp for large groups of vehicles leaving Disneyland, one of the world's most visited attractions. It holds routes both on the deck of the structure and has a crossing route under the structure. The bridge itself crosses over eight lanes of high density traffic routes, four heading south, and four heading north on Interstate-5 with a vertical clearance of $18.5 \mathrm{ft}$. Figure 4 (a) and (b) provide plan view and elevation of the selected bridge. Being a three-span, curved bridge, without skew or base isolation systems, Bridge 55-0837S served naturally as a proper scenario under the scope of this thesis.

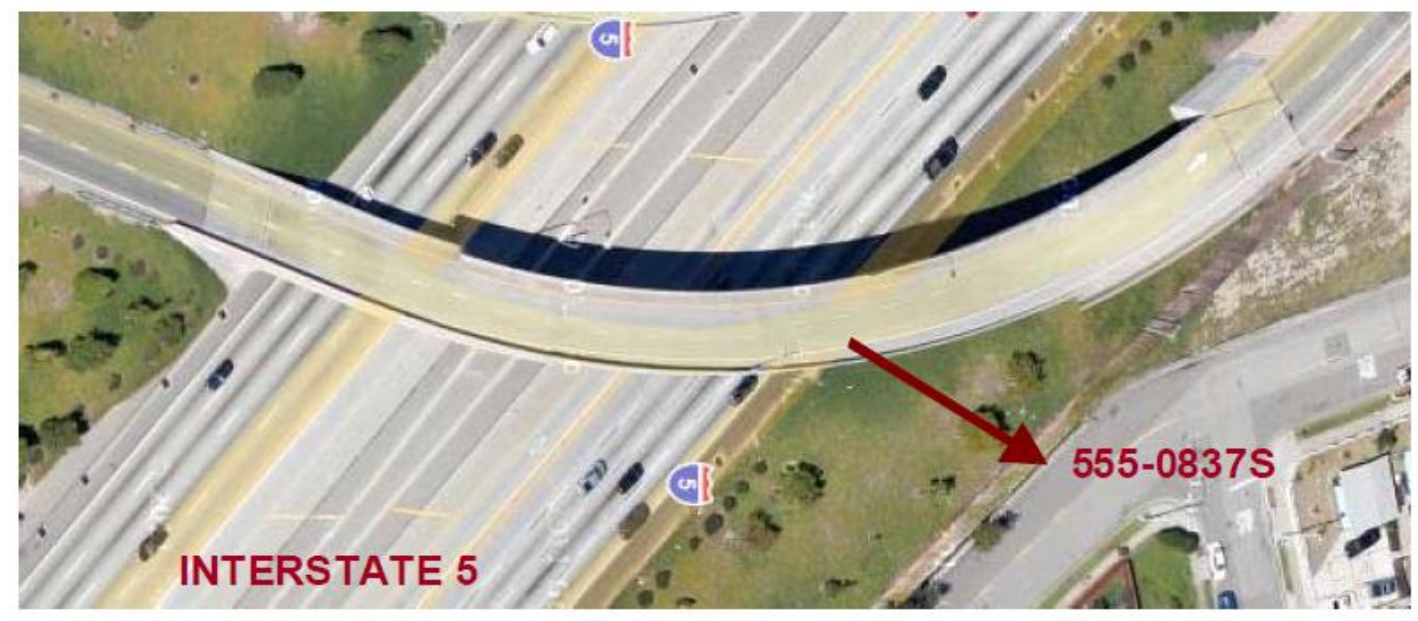

(a) 


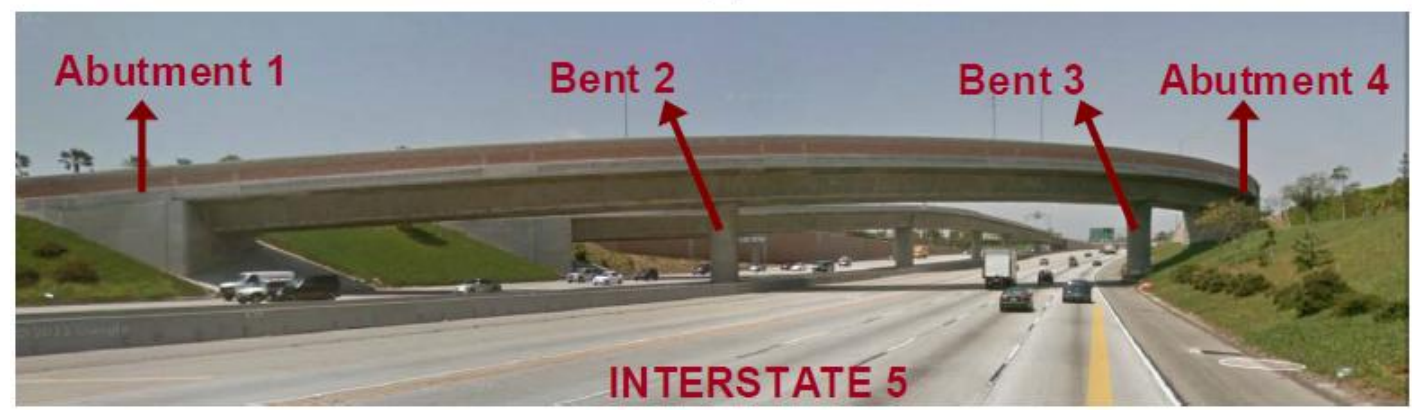

(b)

Figure 4. Bridge 55-0837S (a) plan view and (b) elevation view

\subsubsection{Phase 2: Selection of Ground Motion Histories and Design Spectrum}

As previously mentioned, nonlinear RHA provides an "exact" approach for dynamic analysis of a structural model in a seismic design process. Theory rooted in the nonlinear analysis creates a heightened sensitivity to effects of ground acceleration records being used, making selection of them critical. Previous research done at University California at Berkeley provided ground motion time histories from direct simulation using simplified earth structure and rupture history for each set (Dreger et al. 2007). However for the evaluation at hand, the more desirable approach was use actual recorded motions, scaled for relative source size, and spectral characteristics which will be gone into more detail later. In order to provide a more appropriate scenario of bridges being placed directly atop of fault crossings, ground motions running at a very close proximity to bridge supports were selected, simulated, and analyzed.

Using the Next Generation Attenuation (NGA) database, Caltrans has provided a set of 10 ground motion record sets at which levels of intensity, severity, time step, and location vary. Record sets were filtered to match design criteria set by Caltrans bridge engineers. A design 
spectrum was then produced from the mean of the geometric means of the 10 ground motion record sets to be used to compare its accuracy in representing the total set during analysis.

\subsubsection{Phase 3: Development of Computer Models}

The general computational approach of this investigation was to model the structure from the supporting soils up to the superstructure, apply prescribed earthquake displacements, and solve the equations of motion using three different methods to calculate the bridge's structural response and evaluate the effectiveness of each procedure.

The finite element bridge model developed in Open System for Earthquake Engineering Simulation (OpenSees) (Mazzoni et al. 2006) by the researches from the University of California, Irvine was modified into both linear elastic and nonlinear inelastic models for use in this investigation. Spring systems were assigned to both bent supports and abutments to consider soil structure interactions. Restraining effects due to presence of shear keys, wing walls, and back walls at abutments were also considered in defining spring stiffnesses in each direction.

\subsubsection{Phase 4: Evaluation of Previous Procedures}

The Fault-Rupture Linear Static Analysis (FR-LSA) and Fault-Rupture Response Spectrum Analysis (FR-RSA) procedures had been previously developed by Goel and Chopra (2008a) and validated for simple straight bridge models. As explained by Filippou (1992) the dynamicallyinduced bending moments, shear forces, torsional moments and axial forces are calculated from the modal displacements and stiffness properties of each bridge element. From this theory, computation of vibration member forces is found using vibration displacements, while the dynamic plus quasi-static displacements are utilized for calculating the total member forces. The 
peak quasi-static response in both methods is computed by nonlinear static analysis of the bridge underground displacement offset associated with fault-rupture. In FR-RSA and FR-LSA, the peak dynamic responses are respectively estimated from combination of the peak modal responses using the complete-quadratic-combination rule and linear static analysis of the bridge under appropriate equivalent seismic forces. In the previous investigation by Goel and Chopra (2008a), the procedures being analyzed primarily focused on fault-parallel components, which differ from the current analysis where both fault-parallel and fault-normal components are examined. Both methods were evaluated for accuracy in estimation in comparison to the "exact" nonlinear RHA.

\subsubsection{Phase 5: Evaluation of Analysis Procedures for Practical Use}

The investigation focuses on providing valid and efficient procedures for bridge engineers to implement into design and analysis standards. Ability of methods to adapt to variability in bridge-to-fault orientation and longitudinal stiffness are appraised. Procedures are evaluated and determined if suitable for implementation into design standards and structural analysis software. 


\section{CHAPTER 2: REVIEW OF EXISTING ANALYSIS METHODS}

\subsection{Introduction}

The general computational approach to the current investigation is to model the selected three-span curved bridge from the foundation up to the superstructure, apply prescribed earthquake displacements, and solve the equations of motion to calculate the structures seismic response. The following sections review past, current, and state-of-the-art investigations regarding bridges crossing high seismic fault zones. Goel and Chopra (2008a) have developed and validated simplified analysis procedures for idealized ordinary straight bridges crossing fault-rupture zones. A main purpose of this research is to extend the theories evaluated in these prior investigations to actual curved bridges.

\subsection{Existing Analysis Methods for Bridges Crossing Fault-Rupture Zones}

As presented by the comprehensive research report by Goel and Chopra (2008a), two simplified analysis methods, i.e., FR-LSA and FR-RSA were developed for bridges crossing fault lines. As alternatives to the nonlinear RHA approach, the simplified methods provide predictions of seismic displacement demands. The methods were developed to provide viable peak responses of linearly-elastic "ordinary" bridges for bridge engineers to use in avoidance of the more rigorous nonlinear procedure of response history analysis (RHA). The study was performed with a series of three companion journal papers (Goel and Chopra 2008a, 2008b, 2009a, 2009b) analyzing both linear-elastic and linear-inelastic bridge models. . 


\subsubsection{Theoretical Background: Linear Analysis}

Both FR-RSA and FR-LSA procedures use superposition of peak values from quasi-static $\left(u_{o}^{s}\right)$ and dynamic parts $\left(u_{o}\right)$, to form a total system response $\left(u^{t}\right)$ for simple straight bridge models. Both components $\left(u_{o}^{s}\right)$ and $\left(u_{o}\right)$, are respectively found through: (1) static analysis of the nonlinear bridge model with the peak values of all support displacements Equation (2.1) and (2.2) applied simultaneously; and (2) conducting either the FR-RSA or FR-LSA procedures on the linear bridge model under the fault-normal and fault-parallel ground motion components.

The presented linear analysis procedures use both general multiple-support excitation and proportional multiple-support excitation theories to produce a total peak structural response. The displacement components at support $l$ of a bridge due to fault-rupture motion along the fault-

parallel and fault-normal directions, $u_{g l}^{\mathrm{FP}}(t)$ and $u_{g l}^{\mathrm{FN}}(t)$, may be respectively approximated as:

$$
\begin{aligned}
& u_{g l}^{\mathrm{FP}}(t)=\alpha_{l}^{\mathrm{FP}} u_{g}^{\mathrm{FP}}(t) \\
& u_{g l}^{\mathrm{FN}}(t)=\alpha_{l}^{\mathrm{FN}} u_{g}^{\mathrm{FN}}(t)
\end{aligned}
$$

where $u_{g}^{\mathrm{FP}}(t)$ and $u_{g}^{\mathrm{FN}}(t)$ are the fault-parallel and fault-normal displacement histories of motion at a reference location, and where $\alpha_{l}^{\mathrm{FP}}$ and $\alpha_{l}^{\mathrm{FN}}$ are the proportionality constants for the $l^{\text {th }}$ support. For a bridge crossing strike-slip fault, $\alpha_{l}^{\mathrm{FP}}$ will be equal to +1 for supports on left side of the fault and -1 for supports on right side of the fault, and $\alpha_{l}^{\mathrm{FN}}$ will be equal to +1 for 
supports on both side of the fault. For bridges crossing other types of faults, i.e., faults with other dip and rake angles, values of $\alpha_{l}^{\mathrm{FP}}$ and $\alpha_{l}^{\mathrm{FN}}$ may differ from +1 or -1 .

For ground excitations defined by Equations (2.1) and (2.2), the equations of motion are demonstrated in Equation (2.3):

$$
\mathbf{m} \ddot{\mathbf{u}}+\mathbf{c u} \mathbf{u} \mathbf{k} \mathbf{u}=-\mathbf{m} \mathbf{\imath}_{\mathrm{eff}}^{\mathrm{FP}} \ddot{u_{g}} \mathrm{FP}(t)-\mathbf{m} \mathbf{l}_{\mathrm{eff}}^{\mathrm{FN}} \ddot{u_{g}} \ddot{\mathrm{FN}}^{\mathrm{FN}}(t)
$$

where $\mathbf{m}$, $\mathbf{c}$, and $\mathbf{k}$ respectively represent the mass, stiffness and damping matrices of the system; $\ddot{\mathbf{u}}, \dot{\mathbf{u}}$ and $\mathbf{u}$ respectively represent the acceleration, velocity, and displacement vectors of the bridge; $\mathfrak{l}_{\mathrm{eff}}^{\mathrm{FP}}$ is the "effective" influence vector for fault-parallel motion defined as the vector of displacements at all structural degrees of freedom due to simultaneous static application of all support displacements with value equal to $\alpha_{l}^{\mathrm{FP}}$ at the $l^{\text {th }}$ support of the elastic bridge model, $\mathfrak{l}_{\text {eff }}^{\mathrm{FN}}$ is the "effective" influence vector for fault-normal motion defined as the vector of displacements at all structural degrees of freedom due to simultaneous static application of all support displacements with value equal to $\alpha_{l}^{\mathrm{FN}}$ at the $l^{\text {th }}$ support of the elastic bridge model, and $\ddot{u}_{g}^{\mathrm{FP}}(t)$ and $\ddot{u}_{g}^{\mathrm{FN}}(t)$ are the accelerations at the reference support in the faultparallel and fault-normal directions, respectively.

The total displacements of the bridge are then given by

$$
\mathbf{m} \ddot{\mathbf{u}}+\mathbf{c u}+\mathbf{k} \mathbf{u}=-\mathbf{m} \mathbf{\imath}_{\mathrm{eff}}^{\mathrm{FP}} \ddot{u_{g}^{\mathrm{FP}}}(t)-\mathbf{m} \mathbf{\imath}_{\mathrm{eff}}^{\mathrm{FN}} \ddot{u}_{g}^{\mathrm{FN}}(t)
$$




$$
\mathbf{u}^{t}(t)=\mathbf{u}^{s}(t)+\mathbf{u}(t)=\mathbf{l}_{\mathrm{eff}}^{\mathrm{FP}} u_{g}^{\mathrm{FP}}(t)+\mathbf{l}_{\mathrm{eff}}^{\mathrm{FN}} u_{g}^{\mathrm{FN}}(t)+\sum_{n=1}^{N} \Gamma_{n}^{\mathrm{FP}} \phi_{n} D_{n}^{\mathrm{FP}}(t)+\sum_{n=1}^{N} \Gamma_{n}^{\mathrm{FN}} \phi_{n} D_{n}^{\mathrm{FN}}(t)
$$

in which

$$
\begin{gathered}
\Gamma_{n}^{\mathrm{FP}}=\frac{\phi_{n}^{T} \mathbf{m} \mathbf{\imath}_{\mathrm{eff}}^{\mathrm{FP}}}{\phi_{n}^{T} \mathbf{m} \phi_{n}} \\
\Gamma_{n}^{\mathrm{FN}}=\frac{\phi_{n}^{T} \mathbf{m} \mathbf{v}_{\mathrm{eff}}^{\mathrm{FN}}}{\phi_{n}^{T} \mathbf{m} \phi_{n}}
\end{gathered}
$$

and where $D_{n}^{\mathrm{FP}}(t)$ and $D_{n}^{\mathrm{FN}}(t)$ are the deformation responses of the $n^{\text {th }}$-mode SDF system subjected to the reference ground motions $\ddot{u}_{g}^{\mathrm{FP}}(t)$ and $\ddot{u}_{g}^{\mathrm{FN}}(t)$ in the fault-parallel and faultnormal directions, respectively. The first two terms on right side of Equation (2.5) are the quasistatic response and the last two terms are the dynamic response due to fault-parallel and faultnormal support motions.

The following sections review previous procedures and assumptions for nonlinear RHA, FRRSA, and FR-LSA.

\subsubsection{Nonlinear Response History Analysis}

As previously mentioned, the main target of this investigation is to compare the results produced from FR-RSA and FR-LSA to those calculated by the more rigorous nonlinear RHA. The parametric study included ten sets of ground motion pairs and a design spectrum proposing to accurately represent these sets, as described in detail in Chapter 3, being used as structural excitations while holding the bridge model properties constant per set. RHA is recognized by 
structural engineers to be the most precise in analyzing seismic demands of a structure and is used to generate the "exact" seismic response. It is recognized that while the nonlinear RHA method provides the most accurate results, such an analysis typically requires extensive modeling and computational efforts, which many times is deemed too onerous for common “ordinary” bridges crossing fault zones.

\subsubsection{Fault-Rupture Response Spectrum Analysis}

There are computational advantages in using FR-RSA for prediction of displacement demands in structural systems. The FR-RSA procedure combines the responses of the bridge caused by the fault offset and ground motions, which are respectively determined from the nonlinear static analysis and the response spectrum analysis. The structures displacement caused from this fault offset is created from longitudinal and/or the transverse vector components of the fault offset (called the Design Fault Offset) based on the larger of the probabilistic and deterministic offset.

Proportionality constants $\alpha_{l}^{\mathrm{FP}}$ and $\alpha_{l}^{\mathrm{FN}}$, and peak displacements at reference support location of the bridge $u_{g o}^{\mathrm{FP}}$ and $u_{g o}^{\mathrm{FN}}$ are found and applied to bent supports when forming the quasi-static response of the structure. This quasi-static response is found through static analysis performed on the nonlinear bridge model with fault offsets $\alpha_{l}^{\mathrm{FP}} u_{g o}^{\mathrm{FP}}$ and $\alpha_{l}^{\mathrm{FN}} u_{g o}^{\mathrm{FN}}$ applied at support $l$ in faultparallel and fault-normal directions, respectively. Note that $u_{g o}^{\mathrm{FP}}$ and $u_{g o}^{\mathrm{FN}}$ may have both $\mathrm{x}-$ and y-components depending on the angle between the bridge primary axis and the fault. 
The peak dynamic responses, $r_{o}^{\mathrm{FP}}$ and $r_{o}^{\mathrm{FN}}$, of the linear elastic bridge due to fault-parallel and fault-normal ground hazard are computed as follows:

1. Compute the vibration periods $\left(T_{n}\right)$ and mode shapes $\left(\phi_{n}\right)$ of the bridge. Compute as many modes as necessary to capture dynamic response of the bridge. In general, the first 18 to 24 modes were found to be sufficient in the example structures considered in this study. A complete set of the bridges modes can be found in Appendix B.

2. Compute the fault rupture effective influence vectors, $\boldsymbol{\imath}_{\mathrm{eff}}^{\mathrm{FP}}$ and $\boldsymbol{\imath}_{\mathrm{eff}}^{\mathrm{FN}}$, as vectors of displacements at all structural degrees of freedom due to simultaneous static application of all support displacements with values equal to $\alpha_{l}^{\mathrm{FP}}$ and $\alpha_{l}^{\mathrm{FN}}$ at the $l^{\text {th }}$ support.

3. Compute the modal participation factors for fault-parallel and fault-normal analysis as $\Gamma_{n}^{\mathrm{FP}}=\phi_{n}^{T} \mathbf{m} \mathbf{v}_{\mathrm{eff}}^{\mathrm{FP}} / \phi_{n}^{T} \mathbf{m} \phi_{n}$ and $\Gamma_{n}^{\mathrm{FN}}=\phi_{n}^{T} \mathbf{m} \mathbf{v}_{\mathrm{eff}}^{\mathrm{FN}} / \phi_{n}^{T} \mathbf{m} \phi_{n}$. Note that these modal participation factors differ from those in standard modal analysis to uniform support excitation.

4. Compute the response due to nth mode, $r_{n}^{\mathrm{FP}}$ and $r_{n}^{\mathrm{FN}}$, due to fault-parallel and faultnormal ground hazards using modal analysis and modal participation factors computed in last step.

5. Combine the modal responses, $r_{n}^{\mathrm{FP}}$ and $r_{n}^{\mathrm{FN}}$, due to fault-parallel and fault-normal ground hazards using CQC procedure to obtain peak dynamic responses, $r_{o}^{\mathrm{FP}}$ and $r_{o}^{\mathrm{FN}}$, due to fault-parallel and fault-normal ground hazards, respectively.

This quasi-static responses $r_{\mathrm{QS}}^{\mathrm{FP}}$ and $r_{\mathrm{QS}}^{\mathrm{FN}}$, and the dynamic responses $r_{o}^{\mathrm{FP}}$ and $r_{o}^{\mathrm{FN}}$, are then combined through Equation (2.8) to form a total structural response, $r_{o}^{t}$.

$$
r_{o}^{t}=\left|r_{\mathrm{QS}}^{\mathrm{FP}}\right|+\left|r_{\mathrm{QS}}^{\mathrm{FN}}\right|+r_{\mathrm{O}}^{\mathrm{FP}}+r_{\mathrm{O}}^{\mathrm{FN}}
$$


The Complete Quadratic Combination (CQC) rule is used to combine the peak modal response quantities caused from both fault-normal and fault-parallel ground motion components. As seen in the procedure, modifications from using the standard RSA procedure were taken into account to deal with actual fault-ruptured zones. By using both the modal participation factor, $\Gamma_{n}$ , and an "effective" influence vector, $\boldsymbol{l}_{\text {eff }}$, all of the structures significant modes were able to be evaluated. A complete set of the bridges mode shapes and periods can be found in Appendix B.

\subsubsection{Fault-Rupture Linear Static Analysis}

Goel and Chopra (2008a) evaluated the adequacy of FR-LSA to estimate seismic demands of idealized straight bridges crossing fault-rupture zones. Identical to the FR-RSA procedure, FRLSA estimates the bridge peak response by superposing peak values from quasi-static and dynamic responses. While calculation of the quasi-static response is equal in both procedures they differ in calculating a dynamic response. As presented in the FR-RSA procedure, the quasistatic response is found through static analysis performed on the nonlinear bridge model with fault offsets $\alpha_{l}^{\mathrm{FP}} u_{g o}^{\mathrm{FP}}$ and $\alpha_{l}^{\mathrm{FN}} u_{g o}^{\mathrm{FN}}$ applied at support $l$ in fault-parallel and fault-normal directions, respectively.

FR-LSA was developed through simplifying the FR-RSA procedure by recognizing that the spectral acceleration associated with the periods of the considered modes may be conservatively approximated to be a value equal to 2.5 times the peak ground acceleration (Goel and Chopra 2008a). Such a simplification avoids calculation of the bridge's vibrational period, making it significantly easier and practical for bridge engineers to use. The following steps have been 
provided by Goel and Chopra (2008a) and are applied to the bridge structure to compute the dynamic response, $r_{o}$, in FR-LSA:

(1) Compute effective influence vector $l_{\text {eff }}$, as shown in Figure 5, as the vector of displacements in the structural DOF obtained by static analysis of the bridge due to support displacements determined from Equation (2.1) and (2.2) applied simultaneously.

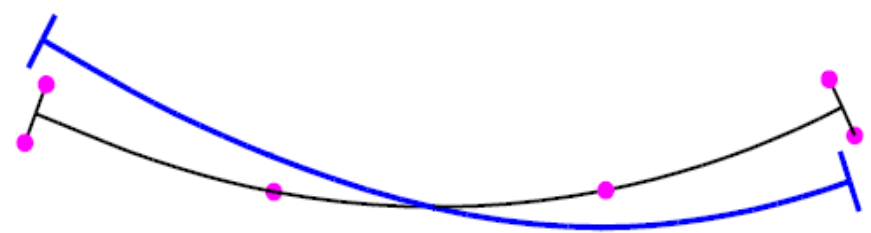

Figure 5. Sketch of the effective influence vector for a bridge crossing fault rupture zones

(2) Compute the dynamic response $r_{o}$ by static analysis of the bridge due to lateral forces $=2.5 m u_{e f f} \ddot{u}_{g o}$.

A final peak response is then calculated through which both the quasi-static response $\left(r_{o}^{Q S}\right)$ from the fault offset in the nonlinear model and the ground motion combination response $\left(r_{o}^{F N . i}+r_{o}^{F P . i}\right)$ from the fault-normal and fault-parallel ground motions in the linear model are combined as shown in Equation (2.9):

$$
r_{o}^{t}=\max \left|r_{o}^{Q S}+r_{o}^{F N . i}+r_{o}^{F P . j}\right|(i=1 \text { or } 2 ; j=1 \text { or } 2)
$$

where:

(1) $r_{o}^{F N .1}$ caused by $2.5 m u_{e f f_{-} F N} \ddot{u}_{g o_{-} F N}$ 


$$
\begin{aligned}
& \text { (2) } r_{o}^{F P .1} \text { caused by } 2.5 m u_{e f f_{-} F P} \ddot{u}_{g o_{-} F P} \\
& \text { (3) } r_{o}^{F N .2} \text { caused by }-2.5 m u_{e f f_{-} F N} \ddot{u}_{g o_{-} F N} \\
& \text { (4) } r_{o}^{F P .2} \text { caused by }-2.5 m u_{e f f_{-} F P} \ddot{u}_{g o_{-} F P}
\end{aligned}
$$

and $m$ represents the mass matrix in the equation of motion; $\iota_{\text {eff }-F P}$ and $l_{\text {eff }} F P$ represent the effective influence vectors for fault-normal and fault-parallel motions; and $\ddot{u}_{g o_{-} F N}$ and $\ddot{u}_{g o_{-} F P}$ respectively represent the peak ground accelerations of the fault-normal and fault-parallel motions.

\subsection{Extension of Previous Study}

It must be noted that the structural systems considered in the investigation provided by Goel and Chopra (2008a) included: (1) a three-span symmetric bridge; (2) a three-span unsymmetric bridge; (3) a four-span symmetric bridge; and (4) a four-span unsymmetric bridge. Each of the bridge models were modeled as being "ordinary" straight bridges with the fault crossing between bent supports and for all bridges, and each of the bent supports modeled as being fixed (restrained in all six degrees-of-freedom). Results of the parametric study successfully provided "accurate" estimates of bridge displacements using all three analysis methods with varying levels

of acceptable precision. This thesis will fill the need of extending these procedures and evaluating them to a real bridge model of an existing curved, three-span bridge with variability in bridge-fault orientations. 


\section{CHAPTER 3: GROUND MOTIONS}

\subsection{Introduction}

Theory rooted in all three RHA, FR-RSA, and FR-LSA procedures makes selection of appropriate ground excitations critical. Several ground motion records were required to prove the adequacy of the analysis methods in extending to varying excitation levels and motion variables. The ground motions used in this thesis were generated and provided by Caltrans in both BridgeNormal (BN) and Bridge-Parallel (BP) directions in order to simulate fault-rupture occurring between bents. The following sections describe the generation and analysis of ground motions as well as the development of the CALTRANS target spectrum.

\subsection{Generation of Ground Motions}

Ground motion pairs were selected to match the design spectrum provided by CALTRANS Seismic Design Criteria (CALTRANS 2010). Using the Next Generation Attenuation (NGA) database, record pairs were filtered to match the following requested CALTRANS criteria:

(1) Records must be from either free-field seismometers or nodes located in the bottom story of a light frame structure.

(2) The lowest usable frequency of either record must be less than or equal to $0.2 \mathrm{~Hz}$ (as specified in the NGA dataset.

(3) The event magnitude must fall within a range of 6.6 to 7.1.

(4) Records must be from sites no closer than $20 \mathrm{~km}$ from the rupturing fault. This requirement was added so that the records would have minimum directivity effect in their signal before the addition of the fling step. 
The record sets chosen were developed from base record pairs that have been amplitude scaled and modified by acceleration pulse. Once integrated into a displacement waveform, the acceleration pulse includes a quasi-static displacement step which represents a specific fault offset also known as a "fling step". A relative fault offset of $100 \mathrm{~cm}$ was found to place bridge bents into the inelastic range while not dominating the contribution of the dynamic response (Shantz and Chiou 2010).

\subsubsection{Selected Record Sets}

Due to the limited number of actual ground motions recorded very close to actual ruptured faults (less than $100 \mathrm{~m}$ ) ground motion simulations are the only method to obtain time histories for structural analysis. These simulated time histories are required to incorporate the near-fault source radiation pattern, and account for far-and near-field seismic radiation during rupture process as well as the sudden elastic rebound (Shantz and Chiou 2010). In total, 200 records were provided by CALTRANS: (10 record sets) x ( 4 orientations +1 no fling record) $\mathrm{x}(2$ components) $\mathrm{x}$ (left or right side of fault $)=200$. The actual final use of the four orientation sets and left and right components are discussed in detail in the following section. Table 1 lists the recorded ground motions that were used for generation of fault rupture ground motions and illustrates the varying levels of intensity, severity, time step, and location.

Table 1. Summary of considered base ground motion pairs*

\begin{tabular}{lllll}
\hline Set No. & Component 1 & Component 2 & Time step (sec) & Number of Points \\
\hline 1 & LOMAP-BVC220 & LOMAP-BVC310 & 0.005 & 5918 \\
2 & LOMAP-HSP000 & LOMAP-HSP090 & 0.005 & 11990 \\
3 & LOMAP-HDA165 & LOMAP-HDA255 & 0.005 & 7928 \\
4 & KOBE-FUK000 & KOBE-FUK090 & 0.02 & 3900 \\
5 & NORTHR-SAR000 & NORTHR-SAR270 & 0.01 & 3600 \\
6 & NORTHR-NEE090 & NORTHR-NEE180 & 0.01 & 4800 \\
7 & KOBE-OSA000 & KOBE-OSA090 & 0.02 & 6000 \\
\hline
\end{tabular}




\begin{tabular}{lllll}
\hline 8 & KOBE-ABN000 & KOBE-ABN090 & 0.01 & 14000 \\
9 & ITALY-A-TDG000 & ITALY-A-TDG270 & 0.0029 & 18216 \\
10 & SFERN-WND143 & SFERN-WND233 & 0.001 & 7997 \\
\hline
\end{tabular}

*selected from the NGA database.

Additional selection considerations included choosing record pairs with minimal scale factors in order to avoid excessive amounts of actual manipulation of amplitudes. The greatest of the 10 record sets scale factors being Record Set No. 4 - Kobe Earthquake with a scaling factor of 13.02 .

\subsubsection{Modification of Set Components}

Problems arose in CALTRANS' attempt to use a functional form for the slip velocity to define the fling step (differentiating the function to acceleration) developed in Dreger (2007). Properties of the functional form resulted in two related and undesirable properties:

(1) A high acceleration spike (approximately $1.5 \mathrm{~g}$ ) at the beginning of the pulse

(2) The acceleration spike resulted in drift when integrated to displacement, presumably due to round-off error. Furthermore, the drift was difficult to remove, presumably due to irregularity related to round-off error.

To avoid these problems, CALTRANS used a functional form of a sine wave to allow for stableness under integration without a drift correction. As represented in Figure 6, the new functional form was used to add a $50 \mathrm{~cm}$ fling step to each of the record sets. 

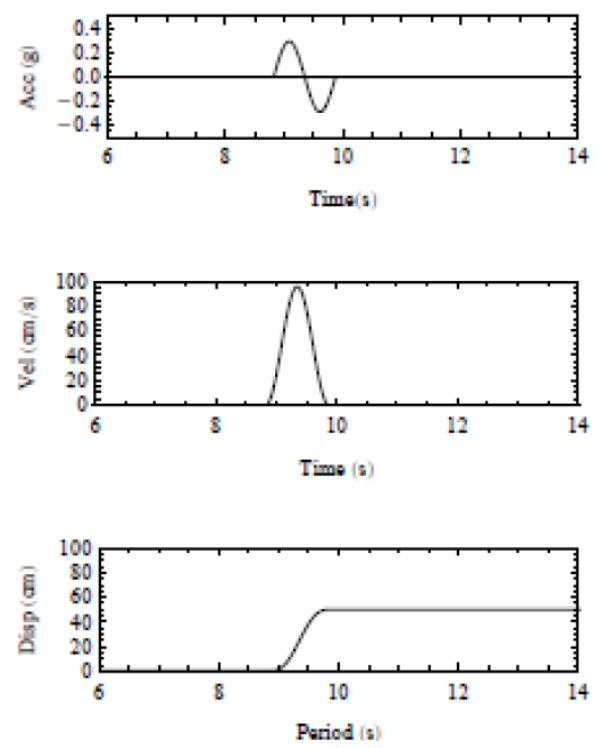

Figure 6. Example acceleration pulse that was added to Record Set 1 to create a $50 \mathrm{~cm}$ fling step (Shantz and Chiou 2010)

Rise time for the rate of displacement offset was modeled by CALTRANS as a log-normally distributed random variable with mean calculated in Dreger (2007) where the rise time was related to the acceleration pulse period as $T_{\text {rise }}=0.60 T_{\text {plse }}$ (Shantz and Chiou 2010). This relationship accounted for initial and final portions of the displacement step relative to the stress drop $\left(\sigma_{L N}=0.4\right)$ varying at a slower pace relative to the middle portion of the step. By doing so a final relation between the periods and fault offset was able to be defined, as illustrated in Figure 7. 


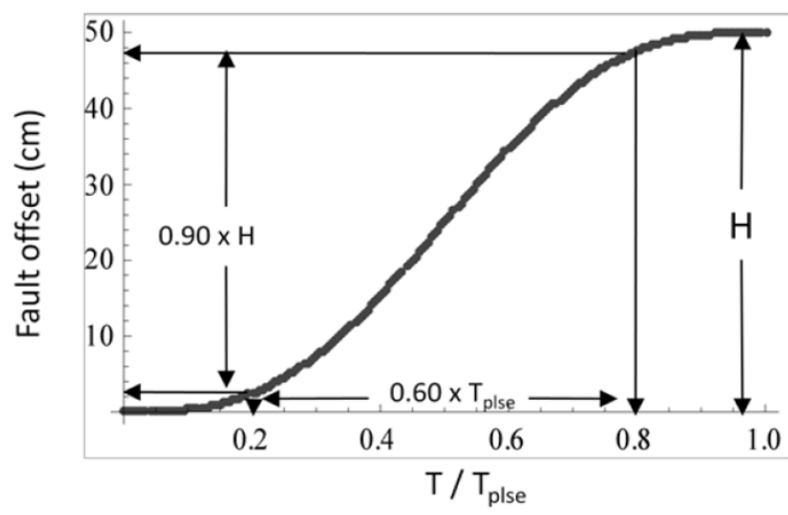

Figure 7. Example acceleration pulse that was added to Record Set 1 to create a $50 \mathrm{~cm}$ fling step (Shantz and Chiou 2010)

Uncertainty in S-wave arrival time in relationship to fault rupture velocity was accounted to by CALTRANS in determining when to add the fling step. This relation was estimated by adding a uniformly distributed random variable ranging from 0.65 to 2.8 seconds to the $\mathrm{S}$-wave arrival time to account for the fact that the faults rupture velocity was seen to be $20 \%$ slower than the S-wave velocity (Shantz and Chiou 2010). The range from 0.65 to 2.8 seconds accounts for the distance between the hypocenter and the bridge along the fault length not matching up, thus having a time difference in arrival.

All record sets have been provided in bridge-normal (transverse) and bridge-parallel (longitudinal) orientations, and further broken down to being on the left side or right side of fault location, e.g. Rec1_BN90_left, Rec1_BN90_right, Rec1_BP90_left, and Rec1_BP90_right. Since the fault-parallel component of shaking is of opposite polarity on each side of the fault, the ground motions, when rotated back to bridge-normal $(\mathrm{BN})$ and bridge-parallel $(\mathrm{BP})$ orientations, are different depending on whether a location is on the left side or right side of the fault (Shantz and Chiou 2010). The time histories for both BP and BN components for Set \#1 are shown in 
Figure 8 and a complete set of time histories and pseudo-acceleration responses can be found in Appendix A.
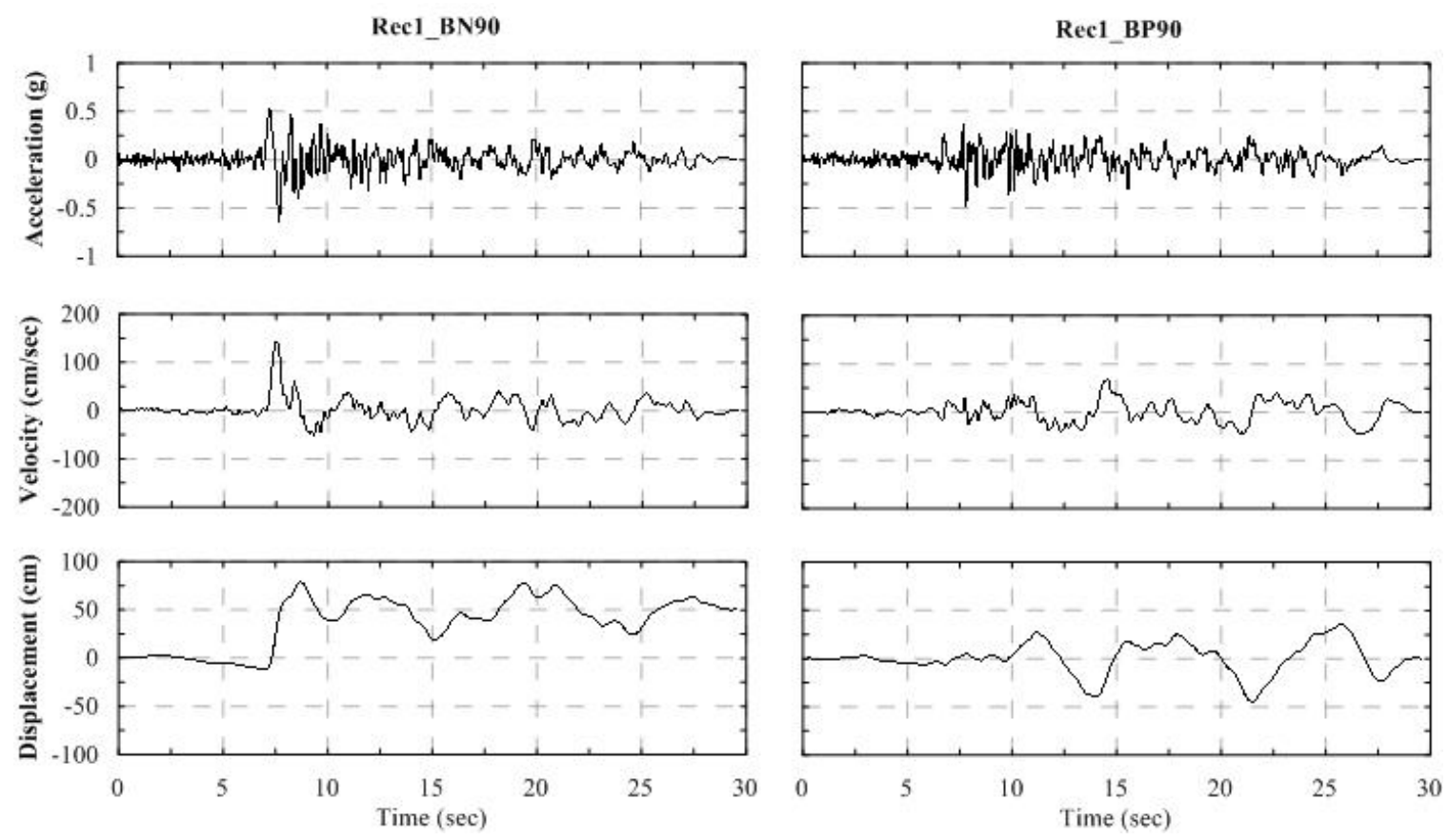

(a)

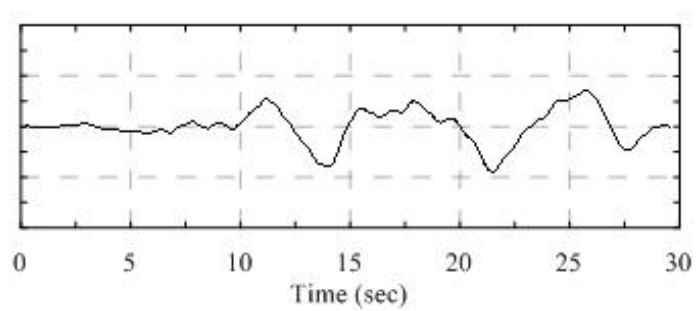

(b)

Figure 8. Time histories for (a) Rec1_BN90 and (b) Rec1_BP90

\subsection{Ground Motion Analysis}

The ground motions in which the bridge model was subjected to include seismic demands from: (1) spatially-uniform ground motions resulting from excitations in bridge-parallel direction (BP); and (2) spatially-varying ground motions resulting from excitations in bridge-normal direction $(\mathrm{BN})$. By including both types of ground motion scenarios, model responses were able to envelope a large spectrum of results, allowing for extension to the remaining bridge scenarios in further investigations. Situations such as the Loma Prieta (1989) and Kobe (1995) earthquakes displayed both types of excitations where pull-off and drop collapses of bridge decks were 
observed due to differential movements between adjacent bridge deck spans (Zanardo et al. 2002). Such scenarios proved that displacements induced by strong ground motions are able to exceed the superstructures design capacity even with pertinent seismic preventions taken. In addition, effects of initial base displacements caused by small differential settlements induce additional further spatial variation acting on the bridge system.

Description of each type of ground motion and application to the bridge model follow.

\subsubsection{Spatially-Uniform Ground Motions}

Uniform ground motions allow for the assumption of unmodified ground accelerations being equally dispersed throughout each of the bridge bent supports. Distribution of the wave propagation is set to be equal for all components of the bridge since the ground motion is taken pre-surface rupture, thus having relatively the same magnitude to all supports. Spatially-uniform ground motions are many times used to simulate bridges as elastic systems when subjected to ground excitations, in contrast to spatially-varying ground motions many times pushing bridge responses into an inelastic response. For this investigation, motions occurring parallel to the bridge are applied as being spatially-uniform since there is no actual fault-rupture being demonstrated between bent supports in this direction. As expected, bridges react in complete different manners when excited by subsurface motions then by surface rupture motions. As presented in the case study done by Wang et al. (2009), using only uniform ground motions can produce 1.07 to 4 times less gap opening in abutments and 0.56 to 20.8 times less at displacement at span joints for long bridges (Wang et al. 2009). 


\subsubsection{Spatially-Varying Ground Motion Across Fault-Rupture Zones}

Since an earthquake excitation consists of superposition of a large number of waves with different characteristics, unless under sub-surface situations as demonstrated in spatially-uniform cases, the different positions along a long-span bridge generally are subject to different motions. For this investigation, motions occurring normal or perpendicular to the bridge are applied as being spatially-varying since they are located between the bridges bent supports and travel with opposite directions. This is seen where motions to the left of the fault (Rec1_BN90_left) are matched with motions occurring in the opposite direction to the right of the fault (Rec1_BN90_right), thus simulating fault rupture. Such non-synchronous ground motions are able to induce responses very different than from using uniform ground motions. In particular, when investigating multi-span systems such as Bridge 550837S, both the varying vibration properties of adjacent spans and the non-uniform spatial ground excitation at the bridge supports can induce differential movements of neighboring decks due to the actual surface rupture of the fault. Such non-synchronization of the super structure can cause a pounding effect between neighboring components if the initial gap is not enough to avoid collision.

\subsection{Development of CALTRANS Target Spectrum}

Previous investigations by Hanks and Bakun (2002) which updated Wells and Coppersmith (1994) equations developed a relation between the mean fault offset and rupture magnitude. The relation by Hanks and Bakun (1994) provided that a magnitude of 6.8 corresponded to a $100 \mathrm{~cm}$ fault offset. Following procedures outlined in CALTRANS SDC 1.6 Appendix B, a target design spectrum based on a vertical strike-slip fault rupturing in a $\mathrm{M}_{\mathrm{w}} 6.8$ event at a zero distance to the 
fault was able to be constructed. As previously described, a near-fault adjustment of $20 \%$ increase in spectral values greater than 1 was also applied to the target spectrum.

As specified by the SDC, the design spectrum (DS) is defined as the greater of the following:

(1) A probabilistic spectrum based on a 5\% in 50 years probability of exceedance (or 975year return period);

(2) A deterministic spectrum based on the largest median response resulting from the maximum rupture (corresponding to max $M$ ) of any fault in the vicinity of the bridge site;

(3) A statewide minimum spectrum defined as the median spectrum generated by a magnitude 6.5 earthquake on a strike-slip fault located 12 kilometers from the bridge site.

By taking the mean of the 10 geometric means for all ground motion sets, one can obtain a smooth target design spectrum compatible to represent the group of 10 record sets. The proposed design spectrum would allow for approximate representation of the entire set of ground motions while shortening the analysis time to a single run per analysis instead of 10 to include each set if needed. Accuracy of using the proposed design spectrum in analysis in comparison to using actual ground motion sets is discussed in detail in Chapters 5 and 6. Figure 9 displays the both the 10 ground motion sets and the proposed design spectrum. 


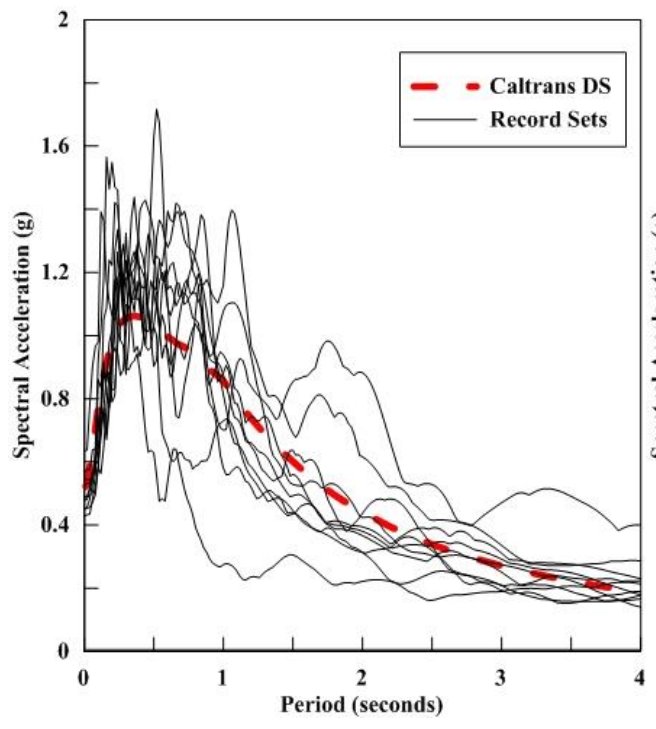

(a)

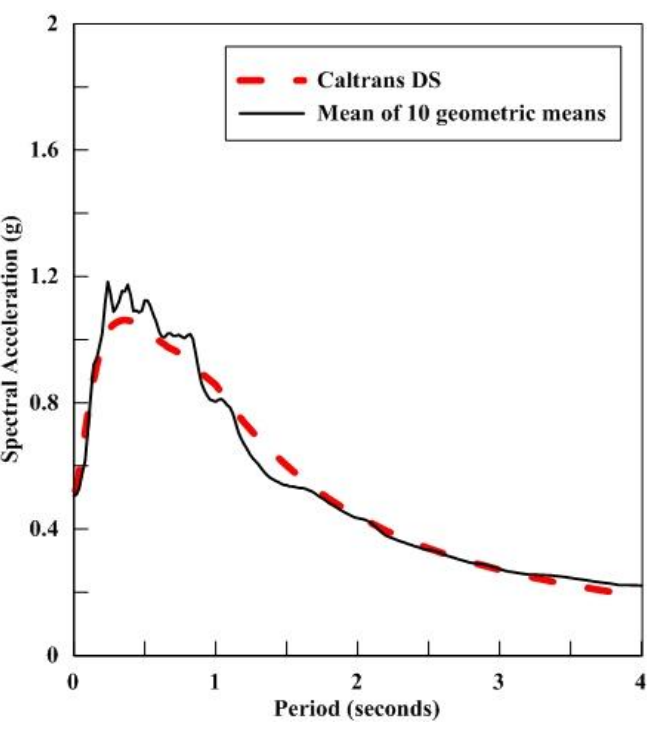

(b)

Figure 9. CALTRANS design spectrum plotted against 10 record sets (a) and against the mean of their geometric means (b) 


\section{CHAPTER 4: DEVELOPMENT OF COMPUTER MODEL}

\subsection{Introduction}

To further verify the adequacy of the two approximate analysis procedures for bridges crossing fault-rupture zones presented in Chapter 2, i.e., FR-RSA and FR-LSA, a bridge model was developed to represent Bridge 55-0837S, and served as the principal bridge being evaluated. The following sections provide basic information, modeling information, and model components for Bridge 55-0837S.

In order to accurately model Bridge 55-0837S, elastic beam-column elements (elasticBeamColumn) and displacement based elements (dispBeamColumn) were used to account for the elements linear and nonlinear responses respectively. Both deck and bent elements were designated six parameters defining their structural integrity including: axial stiffness $(E A)$; bending stiffness about the longitudinal and transverse axes $\left(E I_{l}\right.$ and $\left.E I_{t}\right)$; shear stiffness about the longitudinal and transverse axes $\left(G A_{l}\right.$ and $\left.G A_{t}\right)$; and torsional stiffness $(G J)$ (Ingham et al. 1997). Parallel to the modeling done in Goel and Chopra (2008a) for ordinary straight bridges, the bridge deck elements were modeled as linearly-elastic beam column elements in order to capture the distribution of mass along the length of the deck. Each of the elements were defined by their cross-sectional properties specified based on their fiber section. Element recorders were used to output element forces and deformations through three recording components: stress, strain, and tangent. 


\subsection{Bridge 55-0837S Information}

As previously mentioned, Bridge 55-0837S is a three-span curved bridge built in 2000 in East Anaheim, California (District 12 - Orange County), and serves as the West Street to Northbound Interstate-5 on-ramp. The West Street Bridge serves greatly as a main on-ramp for large groups of vehicles leaving Disneyland, one of the world's most visited attractions. It holds routes both on the deck of the structure and has a crossing route under the structure. The bridge itself crosses over eight lanes of high density traffic routes, four heading south, and four heading north on Interstate-5. According to the data collected in 2000, average daily traffic of Bridge 550837S is about 200,000 vehicles a day (City-Data 2010). Plan and elevation views of Bridge 550837S are demonstrated in Figure 10.

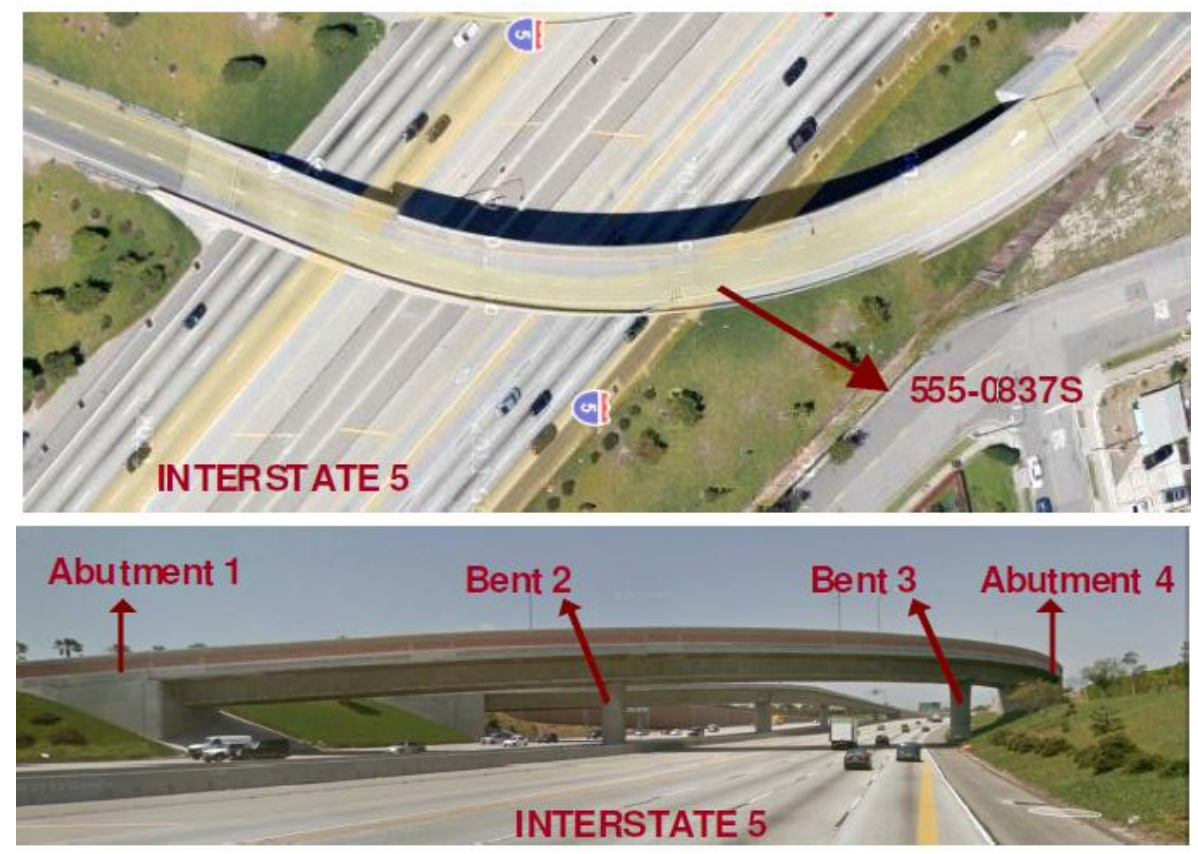

Figure 10. Plan and elevation views of Bridge 55-0837S

(Photos adapted from Google Street View) 
The superstructure consists of a $60.1 \mathrm{~m}$-long center span, a $45.8 \mathrm{~m}$-long left span, and a $45.4 \mathrm{~m}$-long right span. The roadway is $10.8 \mathrm{~m}$ wide from edge to edge, with 5.63 meters of vertical clearance, and accommodates one lane of travel heading north on Interstate-5. Geometries of the bridge are provided in Figure 11.

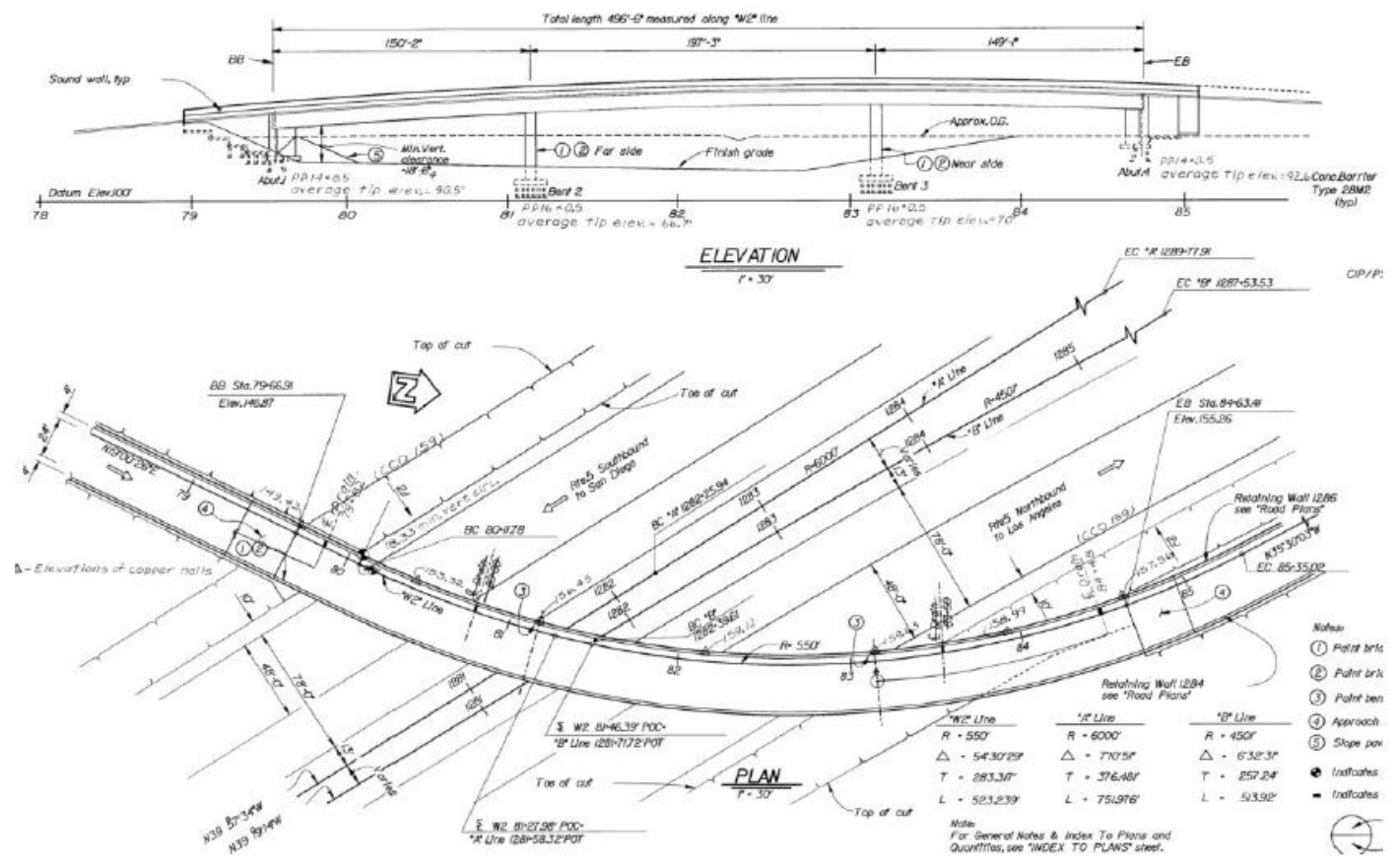

Figure 11. Schematic of Bridge 55-0837S

(Drawings provided by CALTRANS)

The bridges structural components are characterized by CALTRANS as being structural Type 606 in that it is a prestressed concrete continuous bridge (6--) and consists of a single box girder bridge(-06) (Figure 12). Both of the bent supports consist of circular columns consisting mainly of \#4 bar horizontal reinforcement at 12" spacing and 2 layers of vertical \#14 bars for inner support (Figure 13). Bent 2 and 3 are supported by $2 \mathrm{~m}$ deep spread footing reinforced with \#5 rebar at 9" spacing and 69 total piles each (Figure 14). 


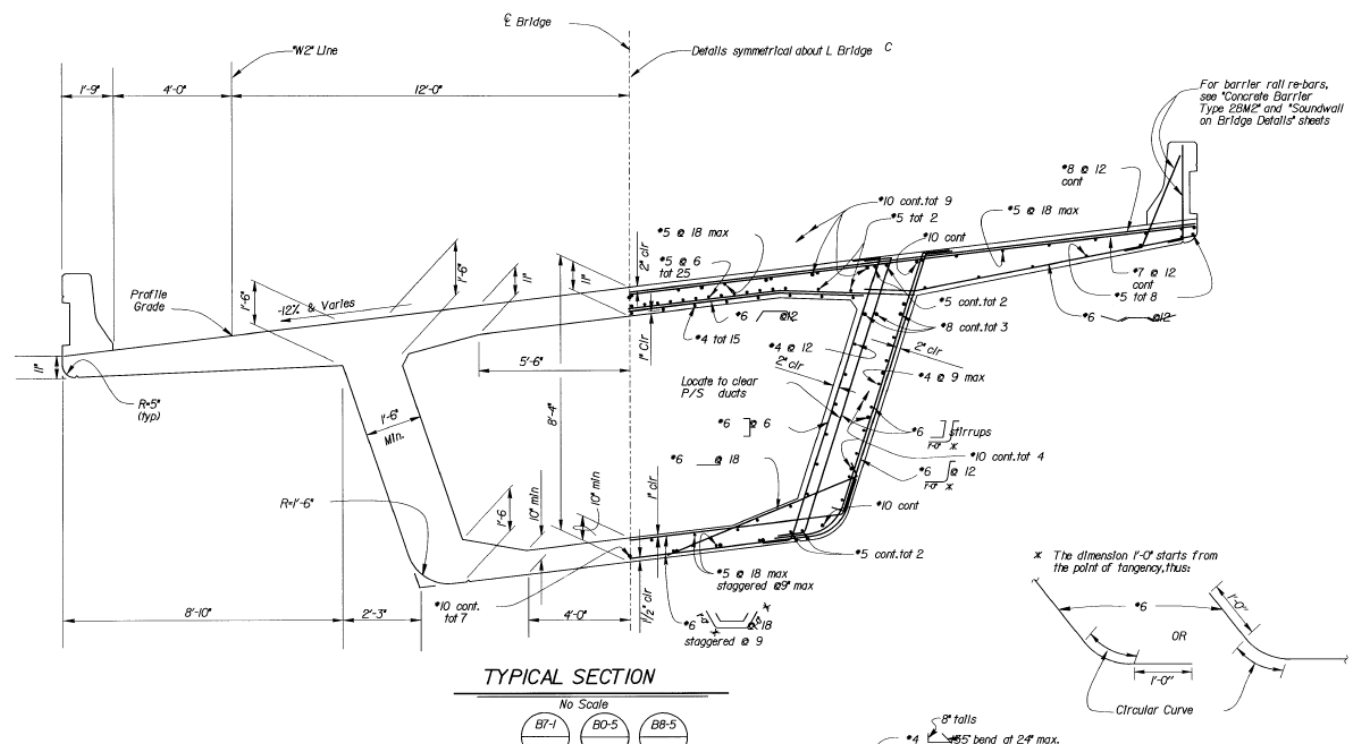

Figure 12. Single box girder typical section for Bridge 55-0837S

(Drawings provided by CALTRANS)

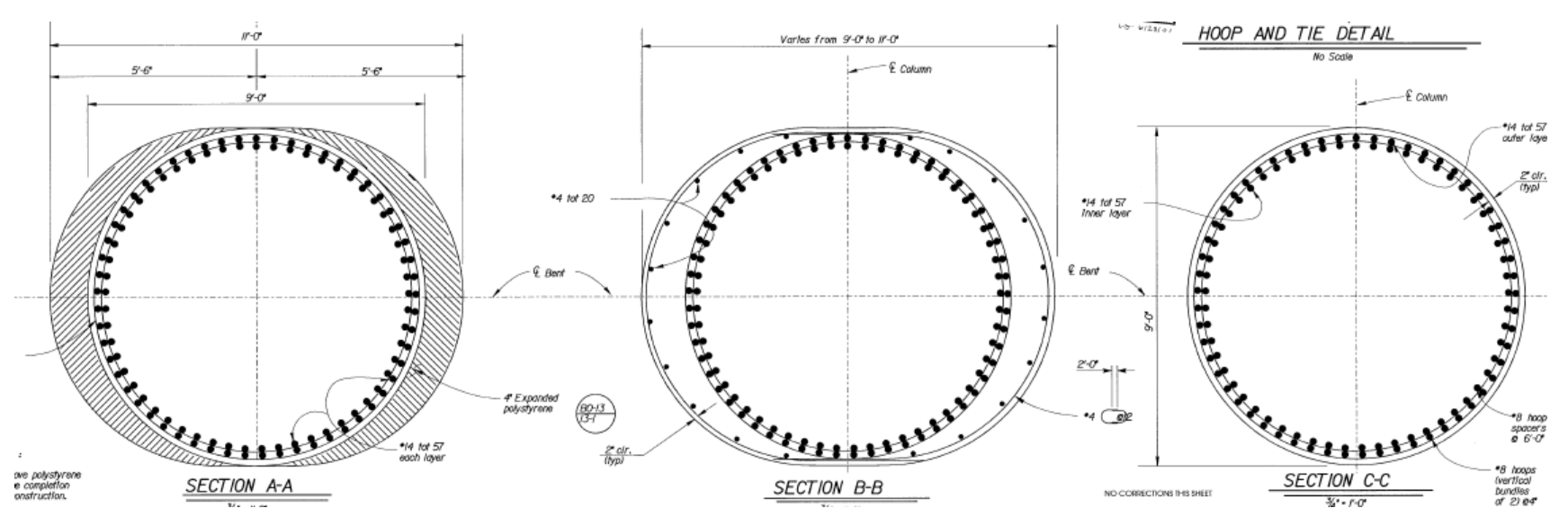

Figure 13. Bent support cross-sections for Bridge 55-0837S

(Drawings provided by CALTRANS) 


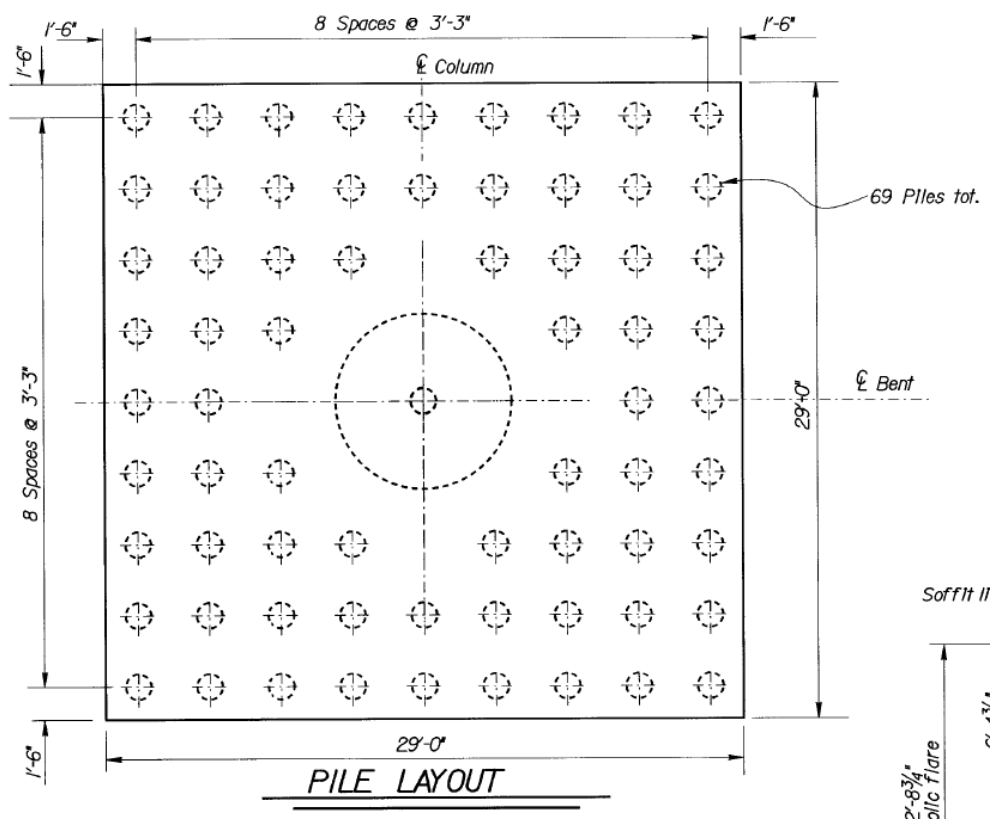

Figure 14. Bent support foundation typical section for Bridge 55-0837S (Drawings provided by CALTRANS)

\subsection{Linear and Nonlinear Models of Bridge 55-0837S}

The finite element model of Bridge 55-0837S was originally developed using OpenSees (Mazzoni et al. 2006) by the researchers from UCI for other research purposes. Both linear and nonlinear models were developed from the original model for use in FR-RSA and FR-LSA procedures in this investigation.

Due to the nature of each of the analyses performed on Bridge 55-0837S, both a linear and nonlinear model were constructed from the original finite element model to satisfy each of the analytical methods. In both the linear and nonlinear models, the bridge deck was modeled using elastic beam-column elements (i.e., elasticBeamColumn in OpenSees). The nonlinear model is identical to the linear model except that the bents were modeled using elastic beam-column 
elements in the linear model, and as beam-column elements with distributed plasticity and linear curvature distribution based on the non-iterative (or iterative) force formulation (i.e., dispBeamColumn in OpenSees) in the nonlinear model.

Consistent with the procedures described in Chapter 2, both the linear and nonlinear models are used for different analysis purposes. The linear bridge model is used by both FR-RSA and FR-LSA in determining the periods, mode shapes, and effective influence vectors of the bridge through eigenvalue and static analysis respectively. The nonlinear model is used in both the FRRSA and FR-LSA procedures to determine the quasi-static response of the bridge. Moreover, the nonlinear model is used in RHA which although time consuming, provides the base results used to evaluate the accuracy of FR-RSA and FR-LSA.

\subsection{Model Components}

The bridge model itself is constructed using a network of nodes and their individual properties defining a series of geometric, coordinate, and material behaviors. Element connections are defined as being continuous across nodal points, i.e., the three translations and three rotations (six degrees of freedom per node), and have stiffness contributions from all elements in contact to that node (Dameron et al. 1997). The structural model is constructed by individual property parameters defined for each of the following model components:

(1) Nodes (2) Masses (3) Materials (4) Sections (5) Elements (6) Load Patterns (7) Time Series (8) Transformations (9) Blocks (10) Constraints 
The finite element model allowed for individual tagging of cross sectional geometries, material behaviors, nodal coordinates, nodal masses, element connections and other parameters for appropriate structural representation. All structural weights were accounted for in the model for determining the seismic response. The following sections go into detail for the bridge's individual components.

\subsubsection{Deck System}

The deck of Bridge 55-0837S was modeled using 30 elastic beam-column elements (i.e., elasticBeamColumn in OpenSees) as shown in Figure 15. The elastic beam-column elements are used to model deck system to ensure it remains elastic under the applied seismic and gravity loads. The cross-section properties determined according to CALTRANS SDC are assigned to each of the deck elements.

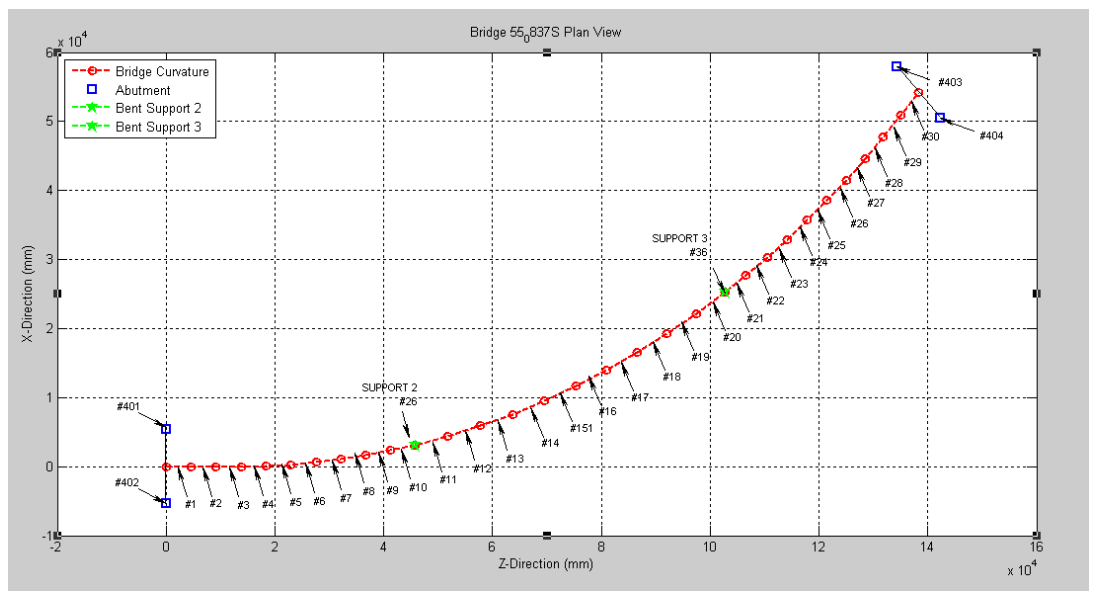

Figure 15. Assignments of nodes and elements in plan view for the FE model of Bridge 55$0837 \mathrm{~S}$

The elastic stiffness of the deck was defined by six principle parameters inside of the model including: axial stiffness $(E A)$; bending stiffness about the longitudinal and transverse axes $\left(E I_{l}\right.$ 
and $\left.E I_{t}\right)$; shear stiffness about the longitudinal and transverse axes $\left(G A_{l}\right.$ and $\left.G A_{t}\right)$; and torsional stiffness (GJ). A summary of the decks property for elements 1-30 is shown in Table 4.1.

Table 2. Summary of deck system properties for deck elements 1-30

\begin{tabular}{|c|c|}
\hline Element Starting Node 1(i) & $\mathrm{i}$ \\
\hline Element End Node 1(j) & $\mathrm{j}$ \\
\hline $\mathbf{A}\left(\mathbf{m}^{\mathbf{2}}\right)$ & 7.6293 \\
\hline $\mathbf{E}(\mathbf{M P a})$ & 276062 \\
\hline $\mathbf{G}(\mathbf{M P a})$ & 23005.2 \\
\hline $\mathbf{J}\left(\mathbf{m}^{\mathbf{4}}\right)$ & 0.2099 \\
\hline $\mathbf{I z}\left(\mathbf{m}^{\mathbf{4}}\right)$ & 0.2099 \\
\hline $\mathbf{I y}\left(\mathbf{m}^{\mathbf{4}}\right)$ & 47.157 \\
\hline
\end{tabular}

\subsubsection{Bent Supports}

Similar to the deck system, each bent was modeled using five elastic beam-column elements (i.e., elasticBeamColumn in OpenSees) in the linear model and five inelastic beam-column elements (i.e., dispBeamColumn in OpenSees) in the nonlinear model as shown in Figure 16. As briefly gone over, the displacement-based beam-column elements in the nonlinear model have a distributed-plasticity and linear curvature distribution using integration along the element based on the Gauss-Legendre quadratic rule (Mazzoni et al. 2006). 


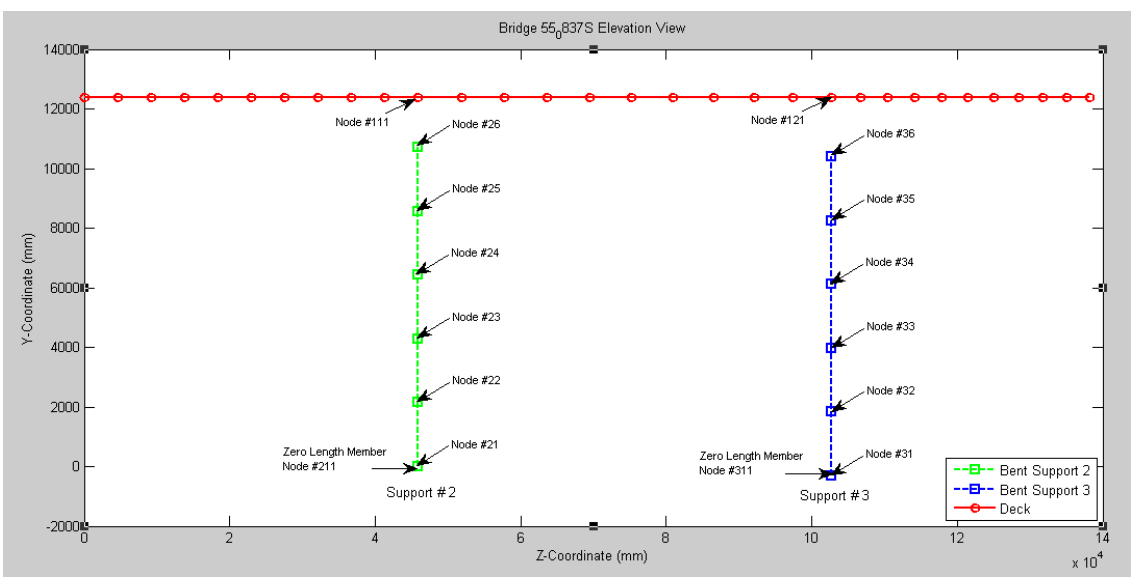

Figure 16. Assignments of nodes and elements in elevation view for the FE model of Bridge 55$0837 \mathrm{~S}$

Soil springs are defined at the bent bases. Consistent with the original UCI bridge model, the rotational and translational springs were assigned stiffness values of $5.65 \times 10^{10} \mathrm{kN}-\mathrm{mm}$ and 145 $\mathrm{kN} / \mathrm{mm}$ respectively; however, the springs along the vertical and torsional directions are assumed to be rigid.

\subsubsection{Abutment Model Design}

Previous investigations have proven the significant importance of abutment behavior, soil structure interaction, and embankment flexibility due to their individual contributions to the total bridge response under moderate to strong intensity ground motions. Aviram et al. (2008) proved that embankment mobilization and the inelastic behavior of the soil material under high shear deformation levels are able to dominate the response of the bridge and the intermediate column bents. In order to ensure correct representation of the abutments, the systems were modeled using linear soil-structure interaction techniques to define: (1) the stiffness of the foundation material; (2) representations of mass and damping of the embedded and enclosed soil; and (3) equivalent masses for the surrounding and enclosed soils (Dameron et al. 1997). The forces in the threedimensional spring-system are obtained from solving three equations of dynamic equilibrium, 
corresponding to two translational motions and one rotational motion about vertical axis of the bridge deck. Mass properties and recorded accelerations provide inertia forces for the bridge deck while stiffness and deformation values of the bents provide their individual forces.

Springs were also assigned at the abutments to consider soil structure interaction and other restraining effects due to presence of shear keys, wing walls, and back walls at abutments. The following defines the vertical, transverse, and longitudinal spring definitions at abutments using CALTRANS SDC recommendations.

\section{Vertical Response:}

Consistent with the original UCI model, the vertical component of the bridges abutment was represented by an elastic spring with stiffness equal to $49,380 \mathrm{kN} / \mathrm{mm}$.

\section{Transverse Response:}

According to Caltrans SDC, the stiffness along the abutment transverse direction should be equal to $50 \%$ of the elastic transverse stiffness of the adjacent bent with consideration of the flexibility of bent foundation. As a result, the linear elastic spring defined along the abutment transverse direction is equal to $20.93 \mathrm{kN} / \mathrm{mm}$.

\section{Longitudinal Response:}

Total bridge displacement in the longitudinal direction is limited by the closure of gaps if the abutment were to be assumed infinitely stiff and strong. However since this is not the case, consideration of additional movements at the abutments due to their flexibility and yielding is important. Both parameters are able to significantly compromise the integrity of the entire bridge system. In addition, movements at the abutments may be due to elastic deformation of the 
backfill soil and slide of the entire system when supported on footings. Other source of movements to be addressed are of that coming from inelastic deformation as a result of yielding in the soil and flexibility of the foundation effecting force transfer due to the yielding soil. Proper estimates of capacity/demand ratios for the different elements are calculated in order to have suitable parameters for soil-structure interactions.

As shown in Figure 17, according to Caltrans SDC (CALTRANS 2010), the stiffness of the equivalent elastic compression-only springs, $K_{\text {eff }}$, is:

$$
K_{\text {eff }}=\frac{P_{b w}}{\Delta_{e f f}}=\frac{P_{b w}}{\Delta_{g a p}+P_{b w} / K_{a b u t}}
$$

where $P_{b w}$ is the passive pressure force resisting movement at the abutment, and where $\Delta_{g a p}$ and $K_{a b u t}$ are the coefficients determined from the elastic-perfectly plastic gap springs defined in the original UCI model. As a result, $K_{\text {eff }}$ was determined to be $28.54 \mathrm{kN} / \mathrm{mm}$.

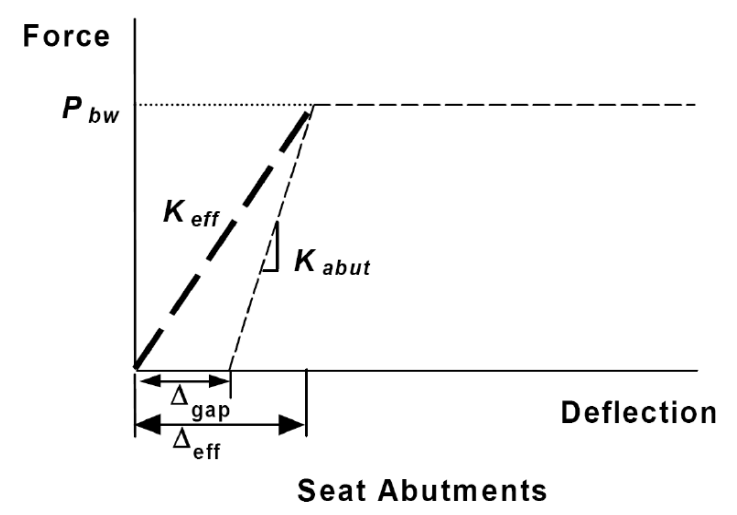

Figure 17. Simplification of longitudinal abutment springs (Adapted from CALTRANS 2010) 
CALTRANS SDC uses this bilinear idealization of the force-deformation relation to account for longitudinal displacements being primarily restrained due to the presence of abutments. This force-deformation relation deduces that larger longitudinal bridge displacements produce more severe damages to abutments. As a result, a smaller stiffness should be assigned to the abutment longitudinal springs to consider the less significant restraining action. The approximation method provides adjustments for assessment of the abutments contribution to the overall bridges seismic performance relative to the bent supports. These adjustments suggest varying the longitudinal abutment spring stiffness from 0.1 to $1.0 K_{\text {eff }}$, which can be further determined from an iterative process based on the longitudinal displacement of the bridge. To validate the FR-RSA and FRLSA procedures for varying abutment contributions to overall systems response, three stiffness values of $0.10 K_{\text {eff }}, 0.55 K_{\text {eff }}$, and $1.00 K_{\text {eff }}$ were considered for the investigation.

\subsubsection{Zero-Length Members}

To ensure the structural integrity and the composite behavior between the bridge deck and bent supports, zero-length members were used to model the connection between joints. In order to capture the structural response and associated damage to bridge connections, modeling of the localized inelastic deformation occurring at the member end regions were identified by shaded areas in Figure 18 (b). Consistent with the OpenSees Command Manual (Mazzoni et al. 2006), these member end deformations consist of two components: (1) the flexural deformation that causes inelastic strains in the longitudinal bars and concrete, and (2) the member end rotation, due to reinforcement slip. This reinforcement slip is the result of strain penetration along a portion of the fully anchored bars into the adjoining concrete members, e.g., deck-to-bent and 
bent-to-footing connections for Bridge 55-0837S (Figure 18 (a)), during the elastic and inelastic response of the structure.

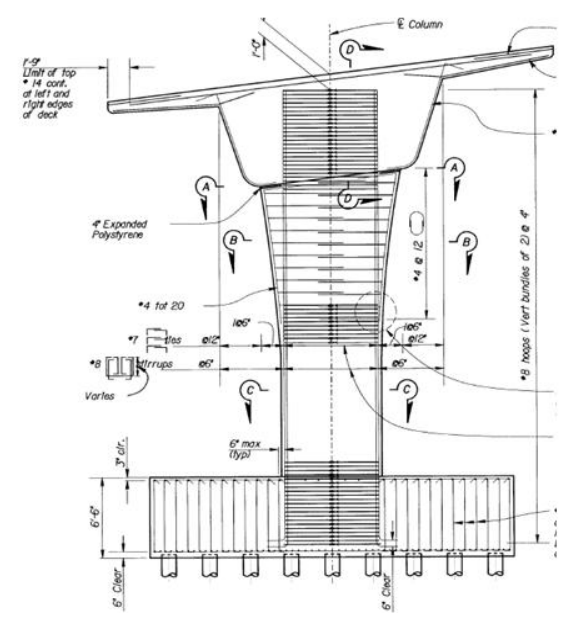

(a)

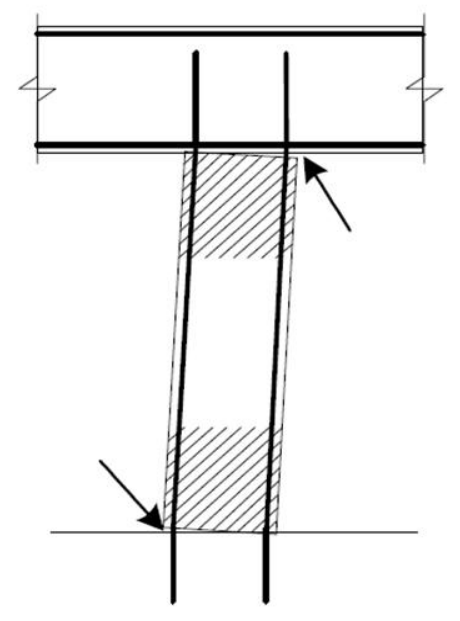

(b)

Figure 18. Actual bent support cross-section (a) and expected inelastic regions at bent support (b) (Adapted from CALTRANS 2010)

To account for these model strain penetration effects (or fixed end rotations), zero-length section members were used. Zero-length elements were placed at intersections between the flexural member and an adjoining member representing a footing or joint and serve as connection two points at the same coordinate, as demonstrated in Figure 19. A list of zero-length elements used in the finite element model is demonstrated in Table 3. 
Table 3. Finite Element Model Zero-length member summary

\begin{tabular}{|c|c|c|c|}
\hline Location & Member \# & Starting Node & End Node \\
\hline \multirow{2}{*}{ Abutment \#1 } & 41 & 411 & 41 \\
\cline { 2 - 4 } Abutment \#2 & 42 & 421 & 42 \\
\cline { 2 - 4 } & 43 & 431 & 43 \\
\hline \multirow{2}{*}{ Bent \#2 } & 201 & 441 & 44 \\
\hline \multirow{2}{*}{ Bent \#3 } & 301 & 311 & 21 \\
\hline
\end{tabular}

The zero-length members allow for a duplicate node (with the same coordinates) to be between a fiber-based beam-column element and the adjoining concrete element. Since the translational degrees-of-freedom from these new nodes are constrained to existing nodes, sliding of the beam-column elements under lateral loads is prevented, due to shear resistance not being included in the zero-length members.

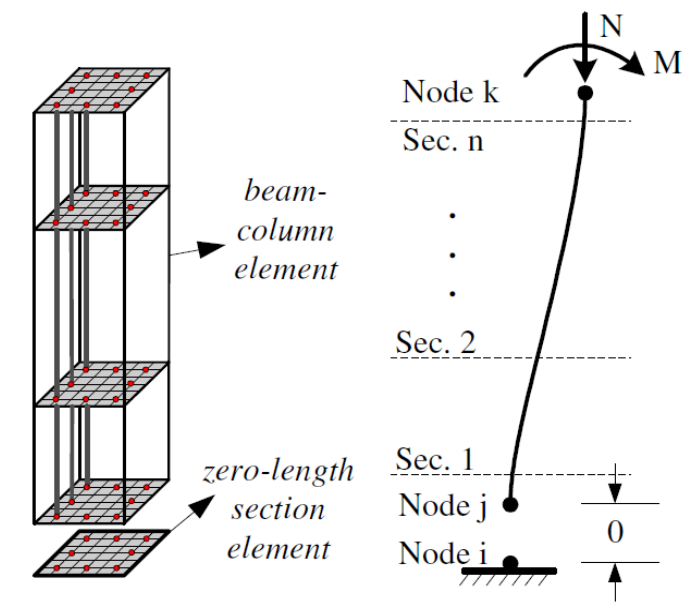

Figure 19. Location of zero-length member to a beam-column element (Adapted from CALTRANS 2010) 
The zero-length members are modeled in OpenSees as having a unit length such that the element deformations (i.e., elongation and rotation) are equal to the section deformations (i.e., axial strain and curvature). The nodes from the zero-length member join the two nodes at a connection by multiple UniaxialMaterial objects to represent the force-deformation relation for the element.

\subsection{Consideration of Bridge Orientations}

To validate the simplified procedures under a wider range of the parameters, it was determined to rotate the bridge orientation to achieve different bridge-to-fault angles and. As shown in Figure 20 (b), the initial orientation of the bridge is assumed to be the one with the chord connecting the two abutments perpendicular to the fault line direction. Moreover, it was assumed at the initial bridge orientation that the two bents are equally spaced on each side of the fault as shown in Figure 20 (b). The angle from the initial orientation to the orientation of interest, $\theta$, is used to define different bridge orientations from the fault rupture line as demonstrated in Figure 20 (a). In this investigation, the clockwise and counterclockwise angles are respectively assigned "+” and "-“ signs. Rotating the bridge about its left abutment, one can determine that the angle, varies between -38 degrees and 53 degrees as shown in Figure 21. It was determined to select the bridge along the following orientation angles: $-53,45,30,21,15$, $0,-15,-30$, and -38 degrees, for further investigation. 

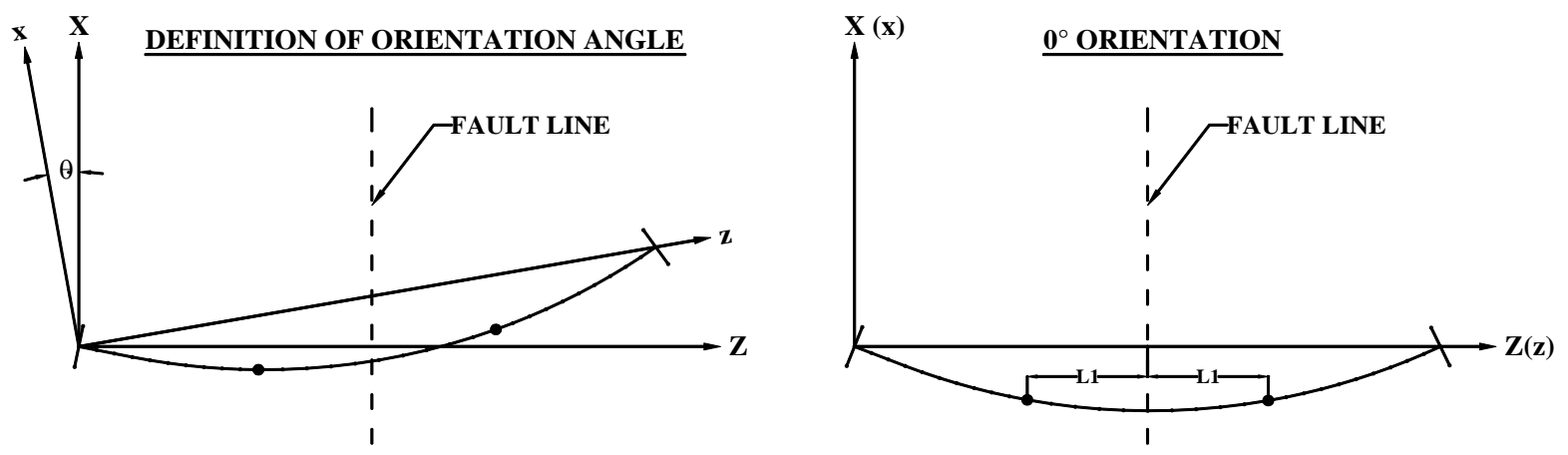

Figure 20. Definition of bridge orientation angle
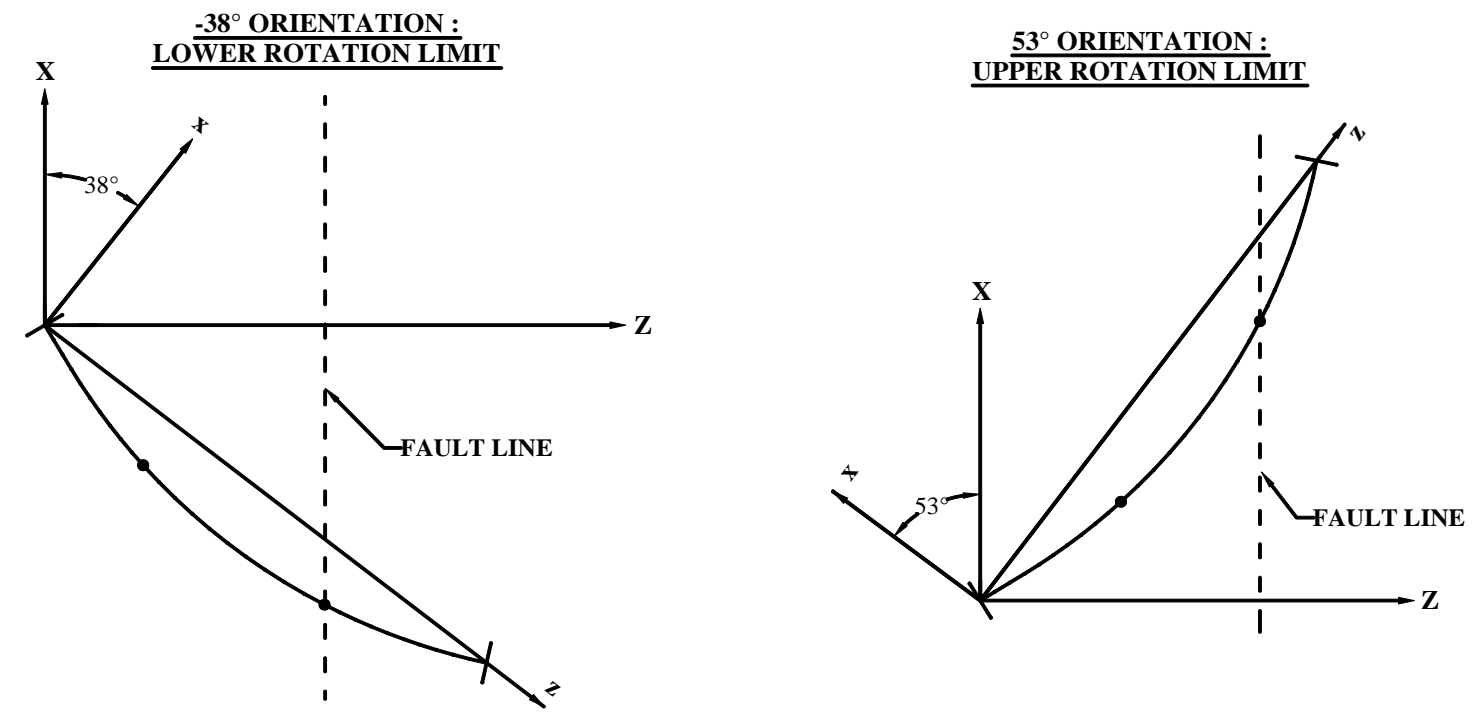

Figure 21. Bridge orientation limit definitions 


\subsection{Response Quantities of Interest}

The response quantities of interest include the displacement demands at the abutments and bents (see Abutments 1 and 4, and Bents 2 and 3 in Figure 10). The maximum absolute values of the abutment longitudinal and transverse displacement components were output for comparison purpose. Abutment displacements in longitudinal and transverse directions were obtained from the transformation of the displacements along global $\mathrm{Z}$ and $\mathrm{X}$ axes as demonstrated in the following equation:

$$
\left\{\begin{array}{l}
z \\
x
\end{array}\right\}=\left[\begin{array}{cc}
\cos \alpha & \sin \alpha \\
-\sin \alpha & \cos \alpha
\end{array}\right]\left\{\begin{array}{l}
z \\
x
\end{array}\right\}
$$

where angle $\alpha$ represents the angle between global and local axes.

In FR-RSA, the abutment longitudinal and transverse displacement components associated with each mode were combined using CQC as described in Section 2.2.2. Moreover, as required in CALTRANS SDC (CALTRANS 2010), the bent rigid body movements caused by foundation deformations were taken into account when calculating the bent displacement demands. The maximum resultant bent displacements were obtained from the square root of the maximum absolute values of the two horizontal bent displacement components and compared in this investigation. 


\subsection{Results}

As discussed in Chapter 4, two parameters, the stiffness of the longitudinal abutment springs (selected to be $0.10 K_{e f f}, 0.55 K_{e f f}$, and $\left.1.00 K_{\text {eff }}\right)$ and the bridge orientation angle $(\theta=53,45$, $30,21,15,0,-15,-30$ and -38 degrees), were varied in the investigation to evaluate the robustness and adequacy of FR-RSA and FR-LSA under the practical ranges of the parameters. Results from the FR-RSA and FR-LSA procedures are differentiated into two categories: ground motions (GM) and design spectrum (DS), which respectively represent the results obtained from:

(1) FR-RSA with pseudo-accelerations for a selected mode computed from elastic RHA of equivalent SDF analysis motion pairs (GM)

(2) FR-RSA with pseudo-acceleration for a selected mode computed from the idealized CALTRANS SDC design spectrum (DS)

(3) FR-LSA with peak ground accelerations selected from each ground motion (GM)

(4) FR-LSA with $2.5 m u_{e f f} \ddot{u}_{g o}$ maximum spectral acceleration from CALTRANS elastic design spectrum (DS)

Moreover, for the results from the GM category, the average values of the bridge responses from the 10 ground motions pairs are presented.

As consistent observations can be obtained from all considered cases, Figures 22 through 24 only present results from $\theta=0$ for all three $0.10 K_{\text {eff }}, 0.55 K_{\text {eff }}, 1.00 K_{\text {eff }}$ stiffness cases. A complete set of results for all orientations stiffness cases can be found in Appendix C. 

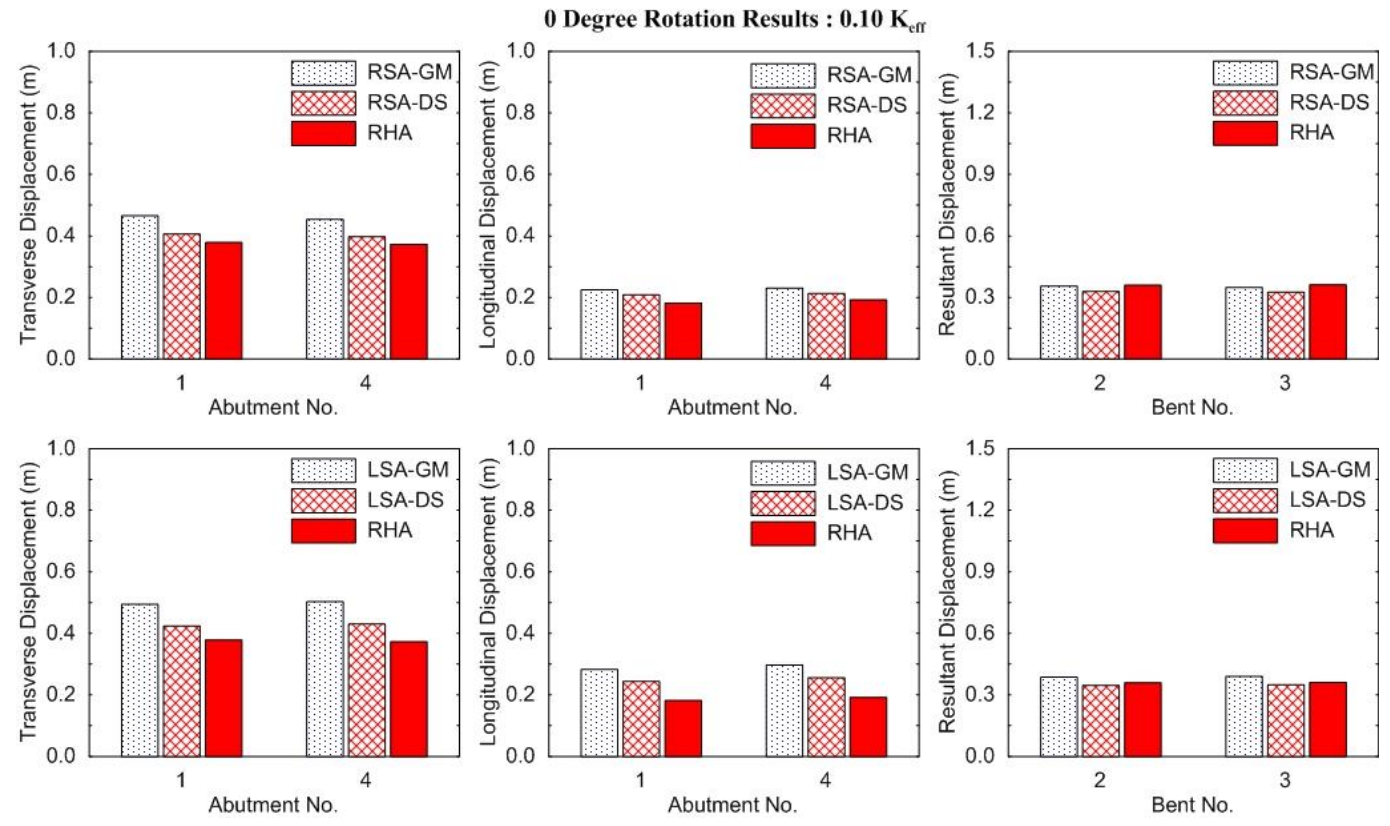

Figure 22. Results for $\theta=0$ degrees and longitudinal abutment stiffness $=0.10 \mathrm{~K}_{\mathrm{eff}}$
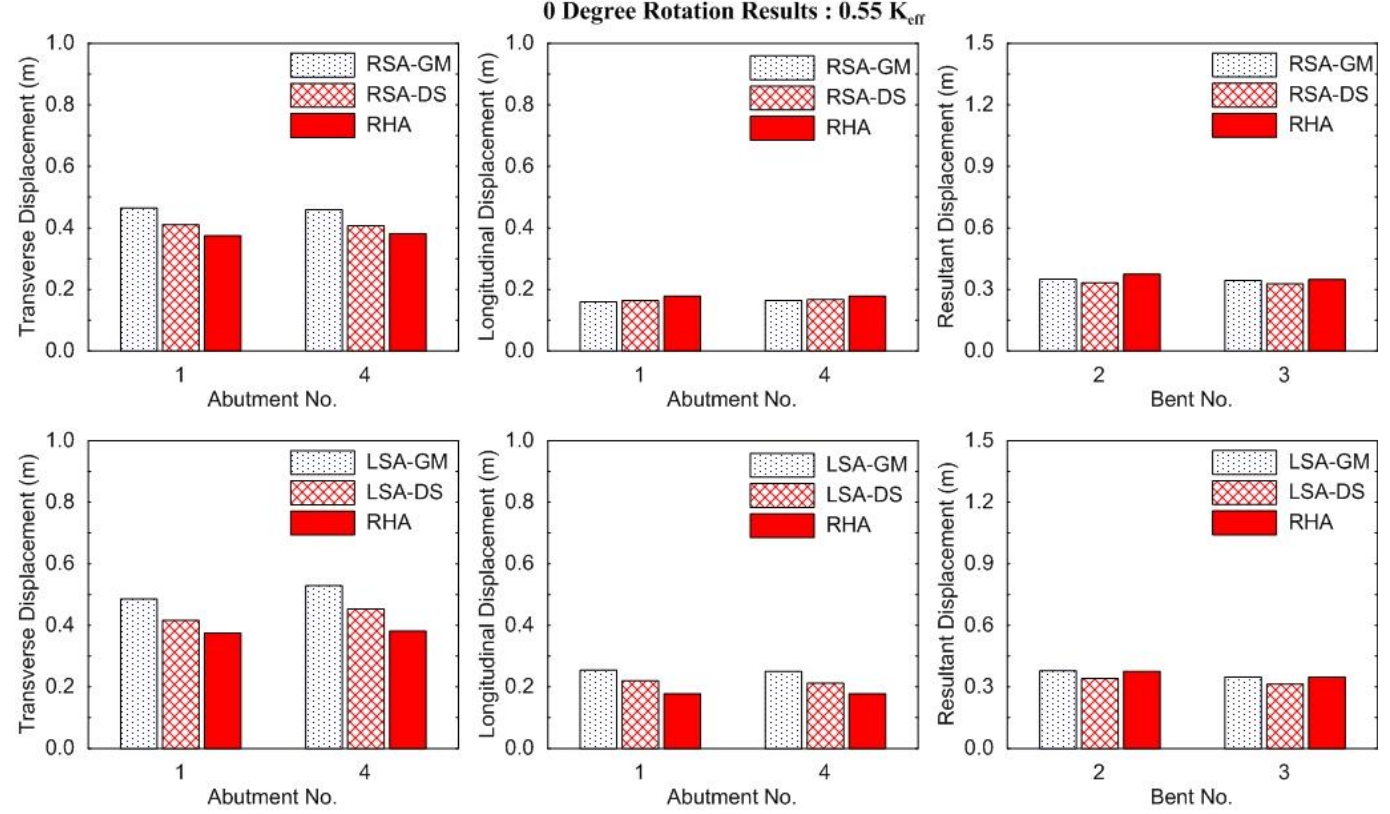

Figure 23. Results for $\theta=0$ degrees and longitudinal abutment stiffness $=0.55 \mathrm{~K}_{\mathrm{eff}}$ 

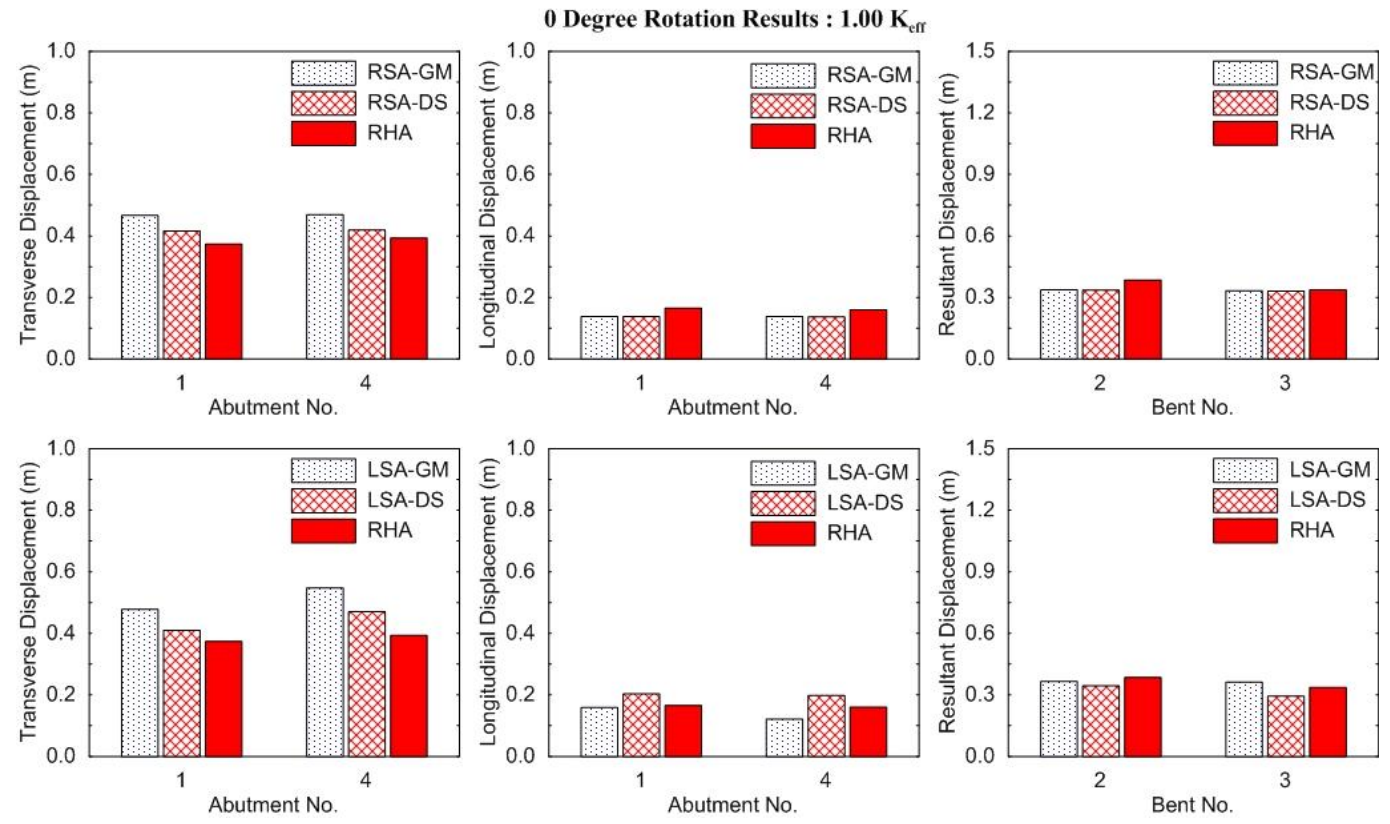

Figure 24. Results for $\theta=0$ degrees and longitudinal abutment stiffness $=1.00 \mathrm{~K}_{\mathrm{eff}}$

\subsection{Discussion of Results}

As presented in Figures 22 through 24 for the $\theta=0$ degree orientation, results consistently show that the FR-RSA and FR-LSA procedures provide reasonable estimates for the seismic demands of the bridge. Discrepancies in results range from $0.29 \mathrm{~m}$ maximum to $0.01 \mathrm{~m}$ minimum for all three $0.10 K_{\text {eff }}, 0.55 K_{\text {eff }}$, and $1.00 K_{\text {eff }}$ stiffness cases. The following sections go into discussion of the results for both abutment and bent support values as well as the accuracy of the procedures in estimating these values:

\subsubsection{Abutments}

For all cases, abutment longitudinal displacements began slightly smaller than transverse displacements for orientations closer to $\theta=0$ and then proportionally grew larger for orientations moving away from the start position. This was expected since the more skewed the 
bridge-to-fault orientations became from its original perpendicular position, the more effect the fault-rupture would have on the bridges longitudinal components, even after being combined through CQC. This is demonstrated in Appendix C (Figures C.2- C.4 and Figure C.34-C.36) where the bridge displays higher longitudinal displacements at its rotation limits of $\theta=53$ and $\theta$ $=-38$. However, regardless of orientation definition or stiffness case, deformations at abutments from RHA were able to be reasonably estimated using FR-RSA and FR-LSA procedures. Accuracy of both procedures for both transverse and longitudinal directions at abutments is presented in Figures 25-28 for all orientation cases.

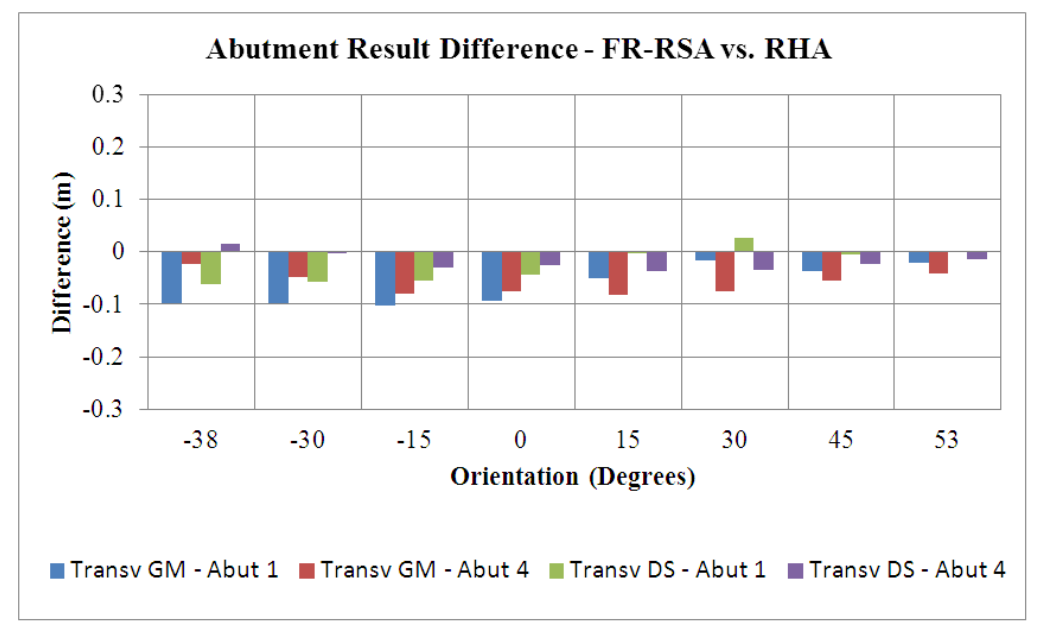

Figure 25. Accuracy of FR-RSA for abutments in transverse direction at all orientations using both GM and DS for longitudinal abutment stiffness $=1.00 \mathrm{~K}_{\mathrm{eff}}$ 


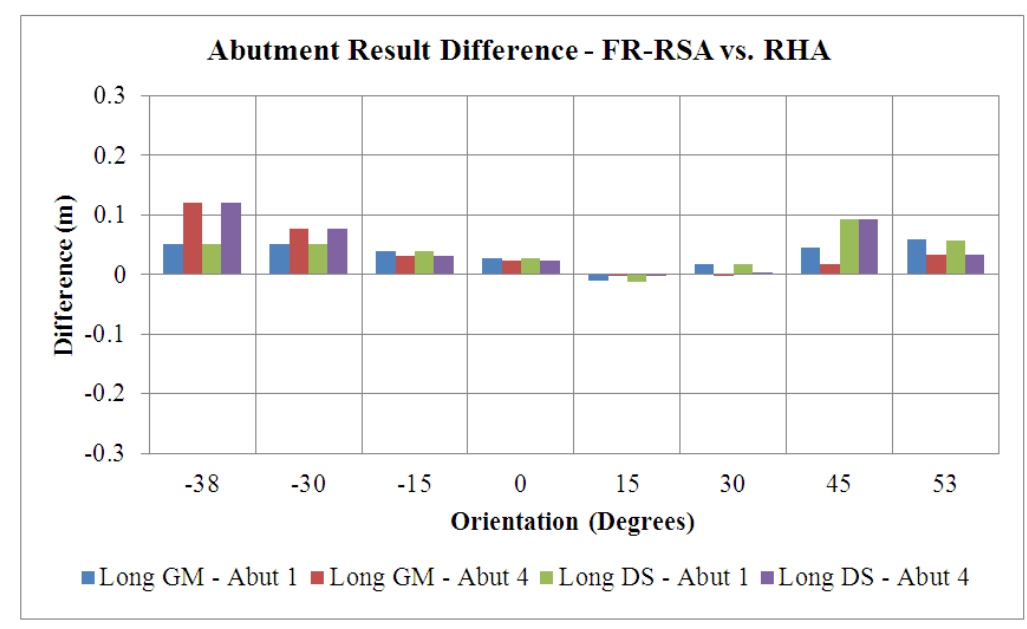

Figure 26. Accuracy of FR-RSA for abutments in longitudinal directions at all orientations using both GM and DS for longitudinal abutment stiffness $=1.00 \mathrm{~K}_{\mathrm{eff}}$

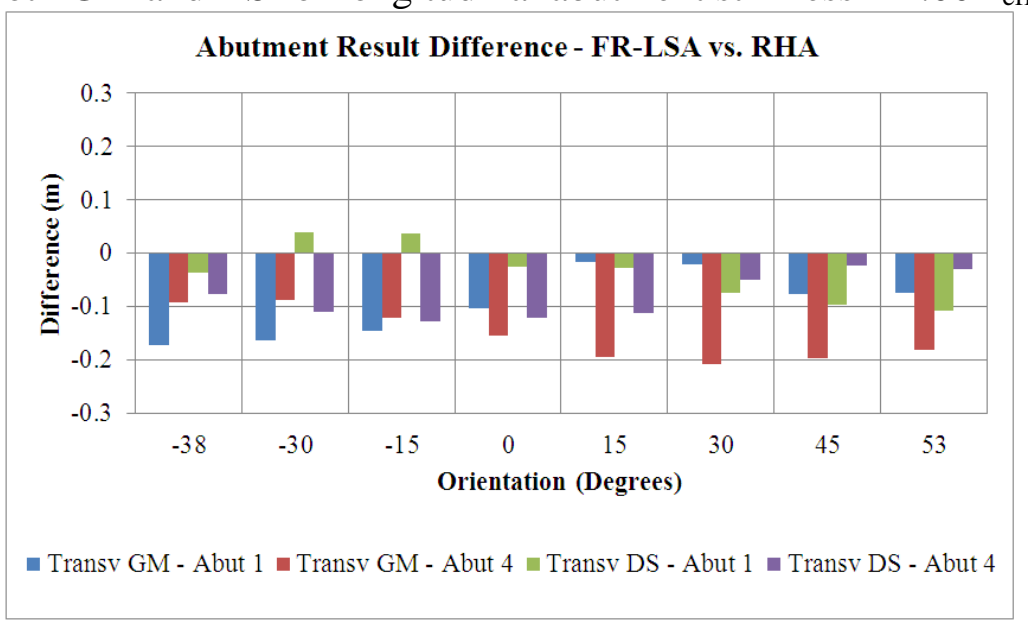

Figure 27. Accuracy of FR-LSA for abutments in transverse directions at all orientations using both GM and DS for longitudinal abutment stiffness $=1.00 \mathrm{~K}_{\mathrm{eff}}$ 


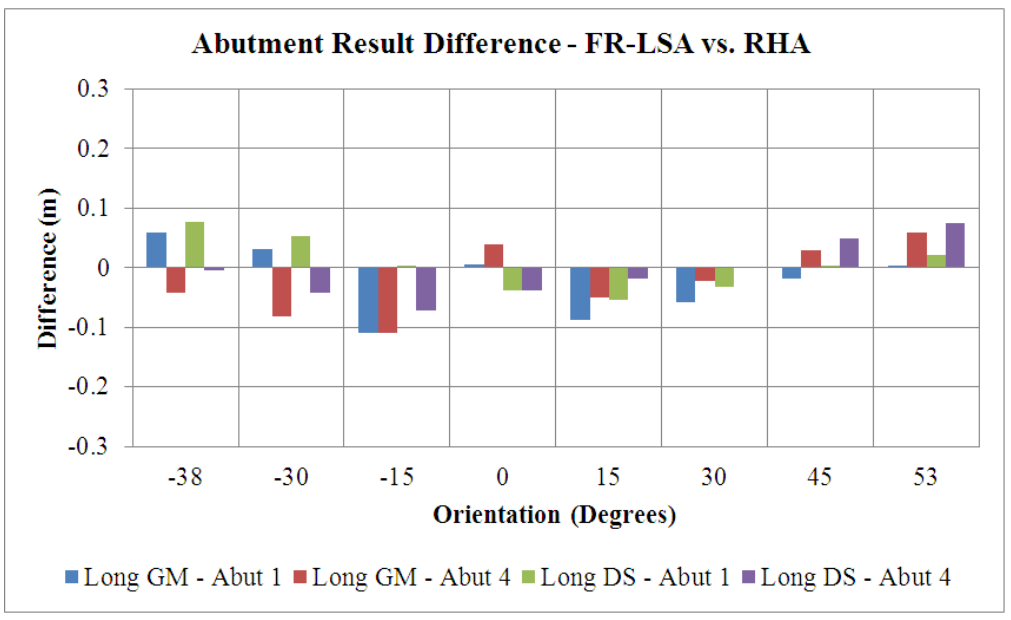

Figure 28. Accuracy of FR-LSA for abutments in longitudinal directions at all orientations using both GM and DS for longitudinal abutment stiffness $=1.00 \mathrm{~K}_{\mathrm{eff}}$

\subsubsection{Bent Supports}

Deformations provided for bent supports were found to be just as accurately approximated using the two procedures as to abutment deformations. In many cases such as for $\theta=-15,0,15$ degrees results at both Bent \#2 and Bent \#3 were found to be considerably accurate, almost identical, to values received from the "exact" RHA procedure. A couple cases displayed slightly conservative deformations for Bent \#2 and slightly underestimated deformations for Bent \#3, as well as vice versa, within the same orientation set. Divergence in values may be attributed to the fault-rupture being located between bent supports and actual influence of the rupture fluctuating between being mostly applied to Bent \#2 or Bent \#3 while moving through rotations. As previously mentioned, the influence of the fault to each bent support is simulated and accounted for through the idealization of spatially-varying excitations as a proportional multiple-support 
excitation in which motions at various supports of the bridge are assumed to be proportional to the motion at a reference location. Accuracy of the two procedures in comparison to RHA for bents is displayed in Figure 29 and Figure 30.

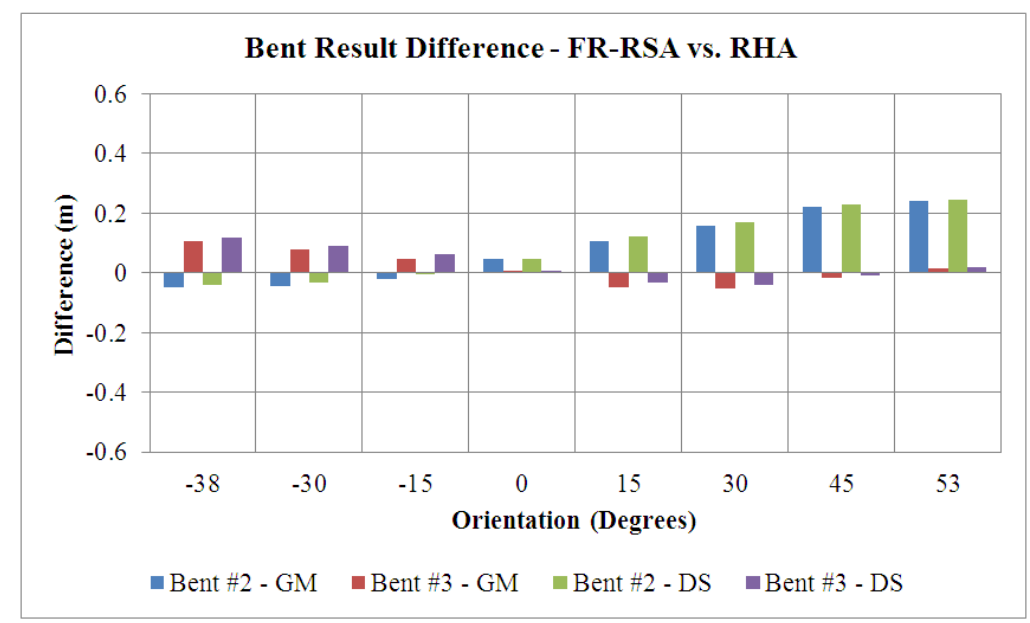

Figure 29. Accuracy of FR-RSA for bents at all orientations using both GM and DS for longitudinal abutment stiffness $=1.00 \mathrm{~K}_{\mathrm{eff}}$

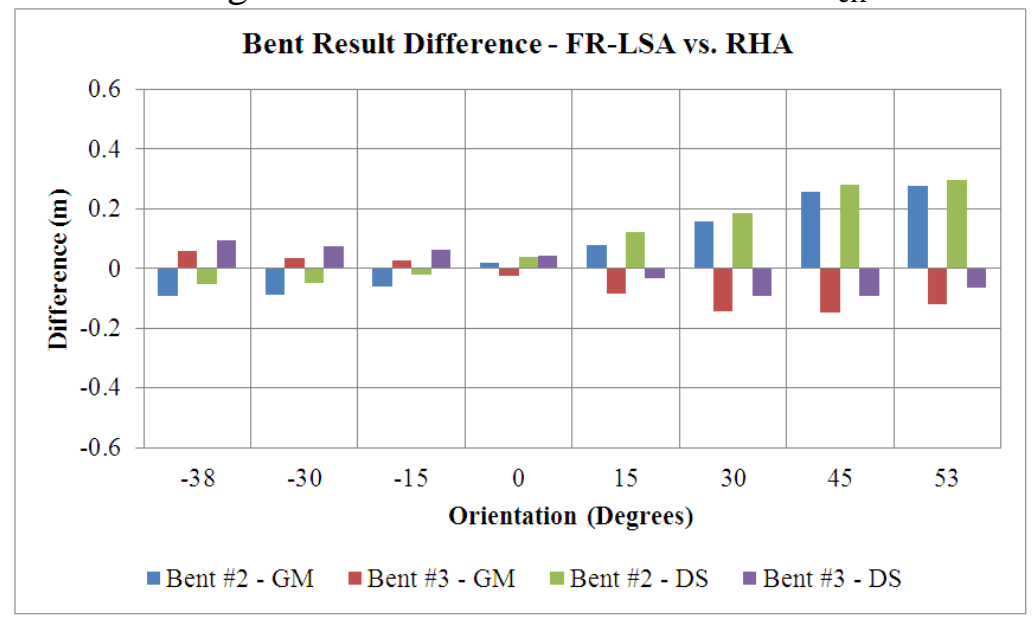

Figure 30. Accuracy of FR-LSA for bents at all orientations using both GM and DS for longitudinal abutment stiffness $=1.00 \mathrm{~K}_{\mathrm{eff}}$ 


\subsubsection{Accuracy of Procedures}

The accuracy of the total (quasi-static plus dynamic) demands for the three-span curved bridge were evaluated for both FR-RSA and FR-LSA proposed approximation procedures. As seen in the results, the proposed FR-RSA procedure is able to consistently show reasonable estimates for the seismic demands of the bridge. As described in Chapter 2, the FR-RSA procedure presented is the most general method of analysis and holds no computational peculiarities related to earthquake engineering analysis (Camata et al. 2007). For all orientations and three stiffness cases, FR-RSA was able to produce deformations within 0.01 to $0.25 \mathrm{~m}$ from the "exact" RHA procedure. These relatively small discrepancies could be attributed to the approximation of using modal participation factors $\Gamma_{n}$, and the computation of the "effective" influence vector $l_{\text {eff }}$ to distinguish all of the bridges significant modes in dynamic analysis. Even with this divergence, there are several cases where FR-RSA generated essentially identical results to the more rigorous RHA procedure.

Similar to FR-RSA, FR-LSA provided reasonably accurate deformation results at both abutment and bent support levels, however doing so while avoiding dynamic analysis. As seen in the Figures 25 through 28 , deformations resulted slightly more conservative than the FR-RSA and RHA procedures primarily due to the use of the conservative estimate of response spectral accelerations. This was the same conclusion as in the study provided by Goel and Chopra (Goel and Chopra 2008a, 2008b, 2009a, 2009b) for ordinary straight bridges. As described in Chapter 2 for FR-LSA procedure, the value of $A$ was conservatively approximated to be 5 times $u_{g o}$, i.e. $A=2.5 u_{g o}$, thus making the peak dynamic response be computed from the application of lateral 
forces equal to $2.5 m l_{e f f} \ddot{u}_{g o}$ applied on the structure. Slightly even simpler than FR-RSA, FR-LSA avoids computation of the vibrational periods of the bridge and the rise-time of the fault-offset. However it must also be noted that there are a couple cases where FR-LSA underestimates the structures seismic response at both abutment and bents such as demonstrated in Figure C. 35 for in Appendix C. 


\section{CHAPTER 6: CONCLUSIONS \& RECOMMENDATIONS}

The presented investigation for analysis of the three-span curved Bridge 55-0837S crossing fault-rupture zones has been implemented through four principal phases: (1) appraisal and analysis of the proposed approximation procedures (FR-RSA and FR-LSA) crossing faultrupture zones in extension to a three-span curved bridge; (2) evaluation of proper ground motion selection and application; (3) modeling of the three-span curved bridge (Bridge 55-0837S); and (4) accuracy of results from procedures in comparison to the "exact" but much more rigorous RHA procedure. Following is the summary of conclusions and recommendations from this investigation:

- One of the principal assumptions in the development of both FR-RSA and FR-LSA procedures was the computation of a total peak value of the bridges seismic response through superposition of peak values from the quasi-static and dynamic parts of the response. As defined in Chapter 2, the peak quasi-static response in both methods is computed by static analysis of the bridge under all ground displacement offsets associated with fault rupture applied simultaneously. In FR-RSA and FR-LSA, the peak dynamic responses are respectively estimated from combination of the peak modal responses using the complete-quadratic-combination rule and the linear static analysis of the bridge under appropriate equivalent seismic forces respectively. Both approximation procedures have great advantages in practicality and application for bridge engineers, such as FR-RSA using the response spectrum directly to account for all significant modes in dynamic analysis, and FR-LSA avoiding computation of the structures vibrational 
periods and estimation of rise time of fault offset through a much simpler static analysis of the bridge under appropriate applied loads. Both procedures were found to provide reasonably accurate results for all $\theta$ definitions and stiffness cases, deeming them valid and practical for use in design and analysis of three-span curved bridges.

- The presented procedures use the idealization of spatially-varying excitations as a proportional multiple-support excitation in which motions at various supports of the bridge are assumed to be proportional to the motion at a reference location. Identical to the conclusion construed for the study provided by Goel and Chopra (2008a), this idealization is judged as being valid for spatially-varying ground motions in close proximity to faults under the varying $\theta$ definitions.

- The finite element nonlinear model of Bridge 55-0837S was able to effectively capture the behavior of the bridge accounting for the Design Fault Offset. While being identical to the linear model, the nonlinear model included plastic hinges and beam-column elements with distributed plasticity and linear curvature distribution based on the noniterative force formulation (i.e., dispBeamColumn in OpenSees). Both FR-RSA and FRLSA procedures computed their quasi-static response using the nonlinear model by applying gravity loads and foundation offsets due to the fault-rupture. The foundation offset was simulated by applying support displacements with values equal to $\alpha_{l}^{F P} u_{g o}^{F P}$ and $\alpha_{l}^{F N} u_{g o}^{F N}$ at support $l$ in fault-parallel and fault-normal directions, respectively. 
- The abutment longitudinal and transverse displacement components associated with each mode were combined using the complete quadratic completion (CQC) rule. Using current CALTRANS SDC (CALTRANS 2010) recommendations for maximum bent displacement demands accounting for bent rigid body movements caused by foundation deformations, a total deformation result was accurately able to be provided through the combination procedure. Moreover, the resultant maximum bent displacements obtained from the square root of the maximum absolute values of the two horizontal bent displacement components were also able to represent the structures final deformation response.

- The fault-rupture "effective" influence vectors at all structural degrees of freedom $l_{\text {eff }}$ used in the procedures was able to properly account for simultaneous static application of all support displacements approximated as $\alpha_{l}^{F P} u_{g o}^{F P}$ and $\alpha_{l}^{F N} u_{g o}^{F N}$ at support $l$ in faultparallel and fault-normal directions, respectively. The dynamic response $r_{Q S}^{F P}$ and $r_{Q S}^{F P}$, of the bridge due to fault-parallel and fault-normal ground motions can be conservatively estimated by the static analysis of the linear structure due to the lateral forced equal to $m l_{e f f}^{F P} A_{\max }^{F P}$ in the fault-parallel direction, and $m i_{e f f}^{F N} A_{\max }^{F N}$ in the fault-normal direction. While in specific cases, the approximation of the peak values of the spectral acceleration for ground hazards $\left(A_{\max }\right)$ resulted in slightly conservative values for the bridges seismic response, overall it was still able to provide reasonably accurate responses for the bridges displacements. 
- By taking the mean of the 10 geometric means, for all ground motion sets, CALTRANS proposed a smooth target design spectrum to represent the group of 10 record sets (Figure 9). The proposed design spectrum would allow for approximate yet valid representation of the entire set of ground motions while shortening the analysis time to a single run per analysis instead of 10 to include each set. The CALTRANS design spectrum (DS) was indeed able to consistently provide an accurate representation of all the ground motions $(\mathrm{GM})$. This stayed true for both abutments and bents for all orientations and stiffness cases, making use of the design spectrum not only valid but efficient. Differences (if any) between results from using GM and DS are seen as negligible for application purposes.

- To ensure the structural integrity and the composite behavior between the bridge deck and bent supports, zero-length members were used to model the connection between joints. Member end deformations consisted of two components: (1) the flexural deformation that causes inelastic strains in the longitudinal bars and concrete, and (2) the member end rotation, due to reinforcement slip. The nodes from the zero-length members joined two nodes at a connection by multiple UniaxialMaterial objects and effectively represented and recorded the force-deformation relation for the bridges elements.

Conclusions from the studies performed by Goel and Chopra (2008a, 2008b, 2009a, 2009b) on idealistic three- and four-span straight bridges perpendicular to faults determined the two principal analysis methods investigated in this paper. The current investigation furthermore proved validity of these procedures to estimate peak seismic responses on a slightly more 
complex three-span curved bridge accurate enough for practical design purposes. Though logistically demanding for bridge engineers, nonlinear response history analysis (RHA) of bridges in this investigation is seen and compared to as being the "exact" solution to the structures dynamic response and is many times needed for bridges on "lifeline" routes, however this procedure may be too onerous for "ordinary" bridges which form a majority of today's infrastructure.

It must be noted that even though the FR-RSA procedure was able to provide valid estimates for the bridge's seismic response, FR-LSA did not require full computation of the bridge's vibration periods or mode effects as compared to the other methods. By simply performing a linear static analysis of the bridge due to appropriate lateral forces, FR-LSA indirectly considers contributions from all vibration modes, ground offset across the fault, and the shape of the response spectrum due to its close proximity to the fault-ruptured zone. Due to the point of the investigation being to find the most simple analysis method while providing accurate results, FRLSA is recommended for further extension onto more complex bridge designs. 


\section{CHAPTER 7: RECOMMENDATIONS FOR SUBSEQUENT STUDY}

While the analysis completed in Year 1 of this project provides better insight on applicability and validity of FR-RSA and FR-LSA in seismic analysis and design of the curved bridges crossing earthquake faults, it is recognized that the bridge example considered in the analysis contains only three spans, for which the bridge displacement caused by the earthquake fault offset is relatively simple due to the absence of interaction of multiple bents on the same side of the fault. To further promote the use of FR-RSA and FR-LSA in practice, it is necessary to evaluate their adequacy in the context of other bridge examples in the future. A possible next focus of work could include analysis of a four-span bridge (Bridge 55-0939G) crossing fault, in which special considerations are given to the fault locations or to a skewed bridge where the effect of the actual fault rupture could alter due to the abutment location.

Subsequent studies could also expand on using nonlinear spring models provided by UC Irvine to represent actual soil-structure interaction systems defining a gap at abutments to serve as a limit of deformation due to unseating or striking effects from excitations. While the transverse spring system used in this investigation is consistent with CALTRANS SDC recommendations, no clear guidelines are provided for modeling of shear keys, effects of separation, impact, and slippage of soil-abutment interfaces. Such effects exhibit highly nonlinear behaviors and could be addressed with more detail in following studies. 


\section{REFERENCES}

1. Afshin, K., \& Amjadian, M. (2010). An Approximate Method for Dynamic Analysis of Highway Bridges with Continuous Rigid Deck. Engineering Structures, 32(9), 28502860.

2. Aviram, A., MAckie, K. R., \& Stojadinovic, B. (2008). Effect of Abutment Modeling on the Seismic Response of Bridge Structures. Earthquake Engineering \& Structural Dynamics, 7(4), 395-402.

3. CALTRANS. (2010). Seismic Design Criteria.

4. Camata, G., Sarnia, A., \& Spacone, E. (2007). Seismic Risk Assesment of a Existing Reinforced Concrete Bridge. Pescara, Italy.

5. Chen, Y. (2001). Nonlinear Seismic Analysis of Bridges: Pracitical Approach and Comperative Study.

6. City-Data. (2010). Retrieved from http://www.city-data.com/bridges/bridges-AnaheimCalifornia4.html

7. Dameron, R. A., Sobash, V. P., \& Lam, I. P. (1997). Nonlinear Seismic Analysis of Bridge Structures: Foundation-Soil Representation and Ground Motion Input. Computers \& Structures, 64(5-6), 1251-1269.

8. Dreger, H. G., Chopra, A. K., \& Larson, S. (2007). Near-Fault Seismic Ground Motions: Earthquake Engineering Research Center.

9. Filippou, F. C. (1992). Nonlinear Static and Dynamic Analysis of Reinforced Concrete Subassemblages.

10. Ghasemi, H. (2004). Bolu Viaduct: Damage Assessment and Retrofit Strategy: FHWA.

11. Ghusn, G. E., \& Saiidi, M. (1986). Simple Histeretic Element for Biaxial Bending of R/C Colulumns (Nonlinear Earthquake Analysis of Bridge Systems). Nevada University, Reno.

12. Goel, R. K., \& Chopra, A. K. (2008a). Analysis of Ordinary Bridges Crossing FaultRupture Zones. University of California, Berkeley, CA: Earthquake Engineering Research Center. 
13. Goel, R. K., \& Chopra, A. K. (2008b). Role of Shear Keys in Seismic Behaior of Bridges Crossing Fault-Rupture Zones. Journal of Bridge Engineering, 13(4), 398-408.

14. Goel, R. K., \& Chopra, A. K. (2009a). Linear Analysis of Ordinary Bridges Crossing Fault-Rupture Zones. Journal of Bridge Engineering, 13(4), 398-408.

15. Goel, R. K., \& Chopra, A. K. (2009b). Nonlinear Analysis of Ordinary Bridges Crossing Fault-Rupture Zones. Journal of Bridge Engineering, 14(3), 216-224.

16. Hanks, T. C., \& Bakun, W. H. (2002). A Bilinear Source-Scaling Model for M-log A Observations of Continental Earthquakes. Seismological American Society(92), 18411846.

17. Honegger, D., Nyman, D., \& Johnson, E. (2004). Trans-Alaska Pipeline System Performance in 2002 Denali Fault. Earthquake Spectra, 20(3), 707-738.

18. Imbsen, R. A., \& Penzien, J. (1986). Evaluation of Energy-Absorption Characteristics of Highway Bridges under Seismic Conditions (Vol. 1): University of California, Berkeley.

19. Ingham, T. J., Rodriguez, S., \& Nader, M. (1997). Nonlinear Analysis of the Vincent Thomas Bridge for Seismic Retrofit. Computers \& Structures, 64(5-6), 1221-1238.

20. Kawashima, K., Takahashi, Y., \& Ge, H. (2008). Damage of Bridges in 2008 Wenchuan, China Earthquake. Tokyo, Japan: Tokyo Institute of Technology.

21. Mazzoni, S., Scott, M. H., \& Fenves, G. L. e. a. (2006). OpenSees Command Language Manual.

22. Nyman, D. J., Johnson, E. R., \& Roach, C. H. (2006). Trans-Alask Pipeline Emergency Response and Recovery. Paper presented at the Sixth U.S. Conference on Lifeline Earthquake Engineering, Long Beach, CA.

23. Shantz, T., \& Chiou, B. (2010). Creation of Record Set for Use in Fault Crossing Study: California Department of Transportation.

24. Toki, K. (1980). Nonlinear Response of Continuous Bridge Subjected to Traveling Seismic Wave. Paper presented at the 7th World Conference of Earthquake Engineering, Istanbul, Turkey.

25. Tseng, W.S., \& Penzien, J. (1975). Seismic Analysis of Long Multiple-Span Highway Bridges. Earthquake Engineering \& Structural Dynamics, 4(1), 3-24. 
26. Wang, J., Carr, A. J., Cooke, N., \& Moss, P. J. (2009). Response of a 344 m long bridge to non-uniform earthquake ground motions. Engineering Structures.

27. Wells, D. L., \& Coppersmith, J. J. (1994). New Empirical Relationships Among Magnitude, Rupture Length, Width, Rupture Area, and Surface Displacement. Seismological American Society(84), 974-1002.

28. Zanardo, G., Hao, H., \& Modena, C. (2002). Seismic Response of Multi-Span Simply Supported Bridges to a Varying Earthquake Ground Motion. Earthquake Engineering \& Structural Dynamics, 31(6), 1325-1345. 


\section{APPENDICES}


Appendix A : Ground Motion Summary 
The following section provides both time histories and pseudo-acceleration responses for Bridge-Normal (BN) and Bridge-Parallel (BP) components, left and right of fault. The following table summarizes the ground motion record sets provided by CALTRANS and used in this investigation.

Table Summary of considered base ground motion pairs*

\begin{tabular}{lllll}
\hline Set No. & Component 1 & Component 2 & Time step (sec) & Number of Points \\
\hline 1 & LOMAP-BVC220 & LOMAP-BVC310 & 0.005 & 5918 \\
2 & LOMAP-HSP000 & LOMAP-HSP090 & 0.005 & 11990 \\
3 & LOMAP-HDA165 & LOMAP-HDA255 & 0.005 & 7928 \\
4 & KOBE-FUK000 & KOBE-FUK090 & 0.02 & 3900 \\
5 & NORTHR-SAR000 & NORTHR-SAR270 & 0.01 & 3600 \\
6 & NORTHR-NEE090 & NORTHR-NEE180 & 0.01 & 4800 \\
7 & KOBE-OSA000 & KOBE-OSA090 & 0.02 & 6000 \\
8 & KOBE-ABN000 & KOBE-ABN090 & 0.01 & 14000 \\
9 & ITALY-A-TDG000 & ITALY-A-TDG270 & 0.0029 & 18216 \\
10 & SFERN-WND143 & SFERN-WND233 & 0.001 & 7997 \\
\hline
\end{tabular}

selected from the NGA database. 

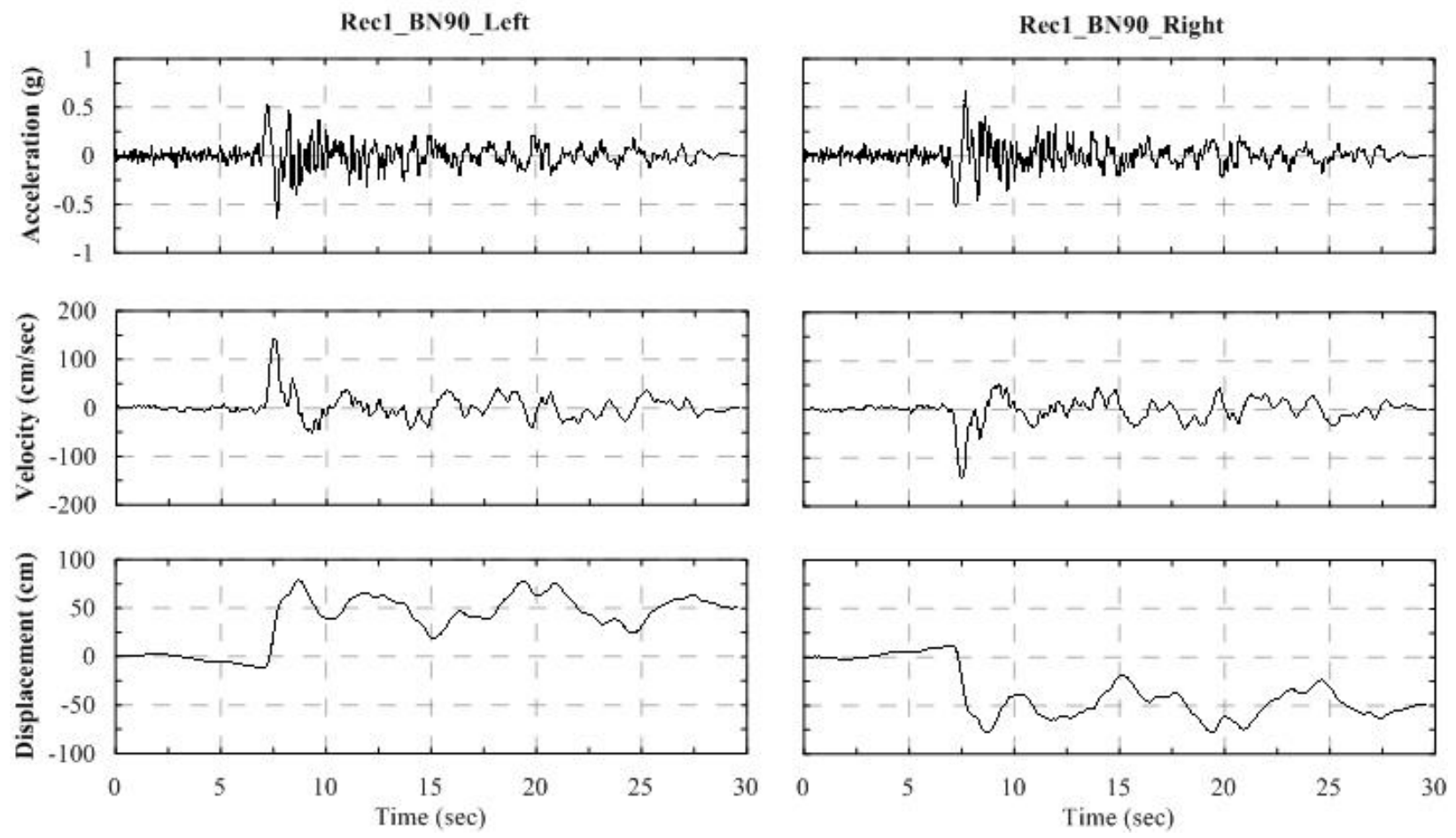

Figure A.1 - Time series summary for Record Set 1 in Bridge-Normal direction
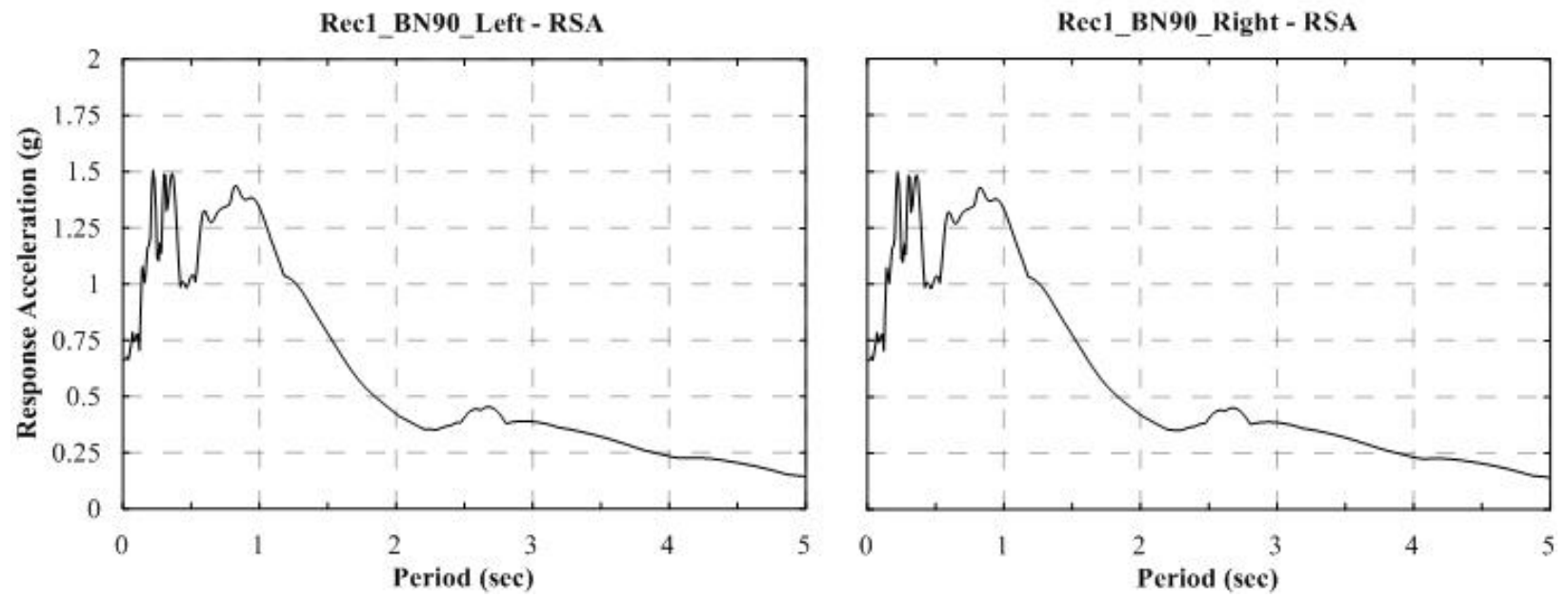

Figure A.2 - Pseudo-Acceleration Response Summary for Record Set 1in Bridge-Normal direction 

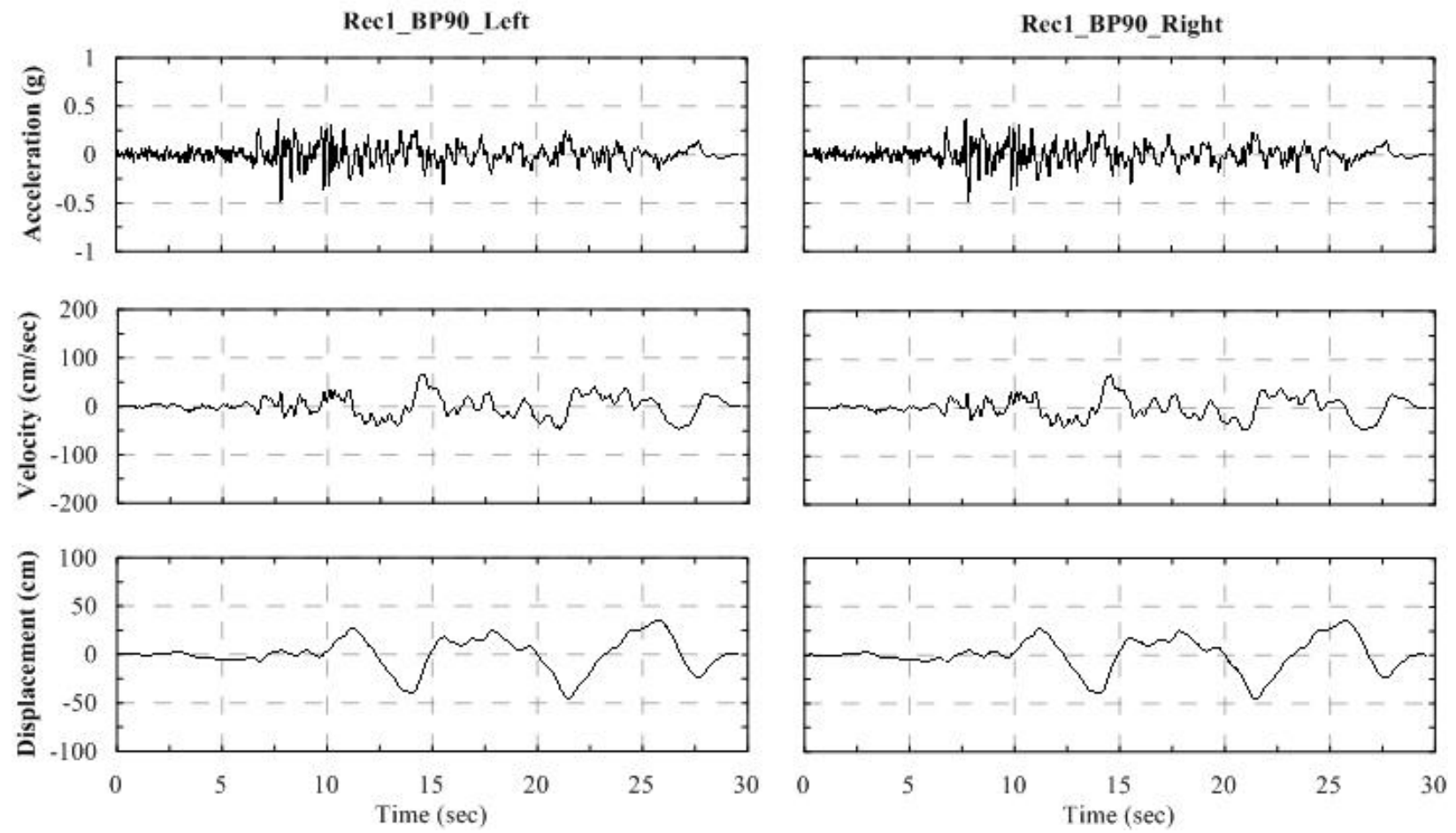

Figure A.3 - Time series summary for Record Set 1 in Bridge-Parallel direction
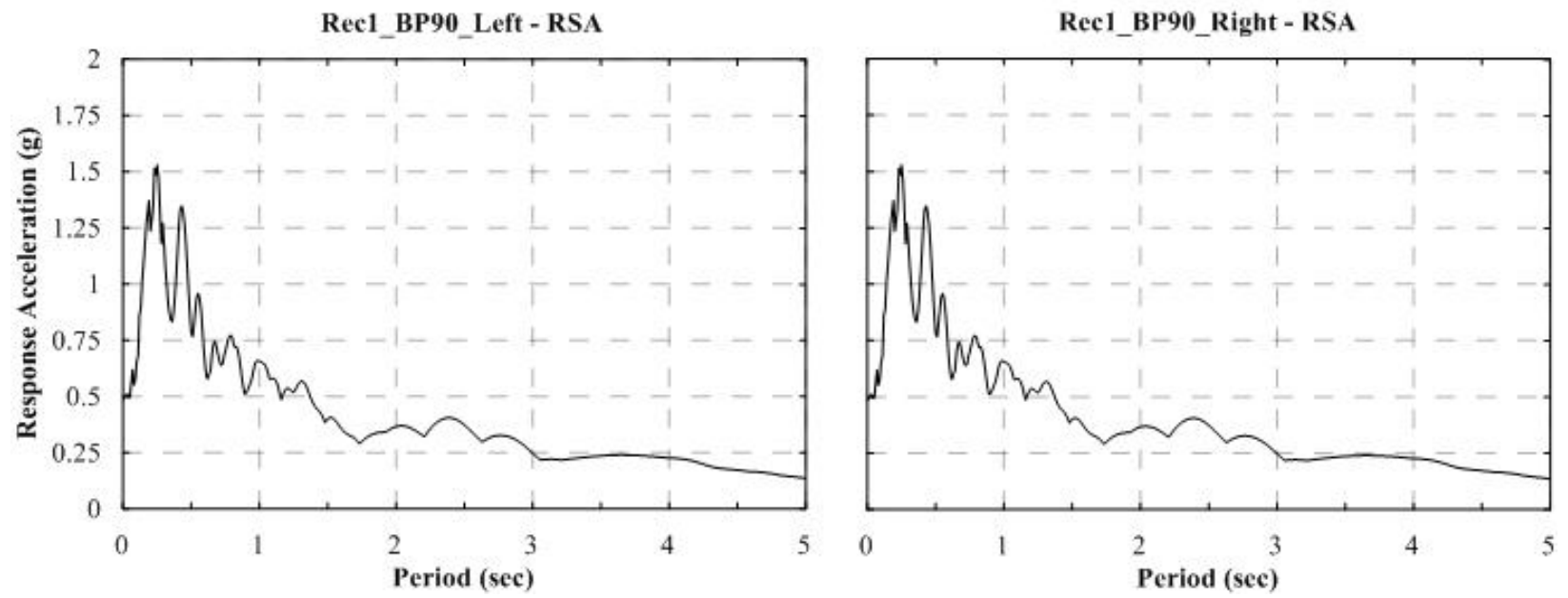

Figure A.4 - Pseudo-Acceleration Response Summary for Record Set 1in Bridge-Normal direction 

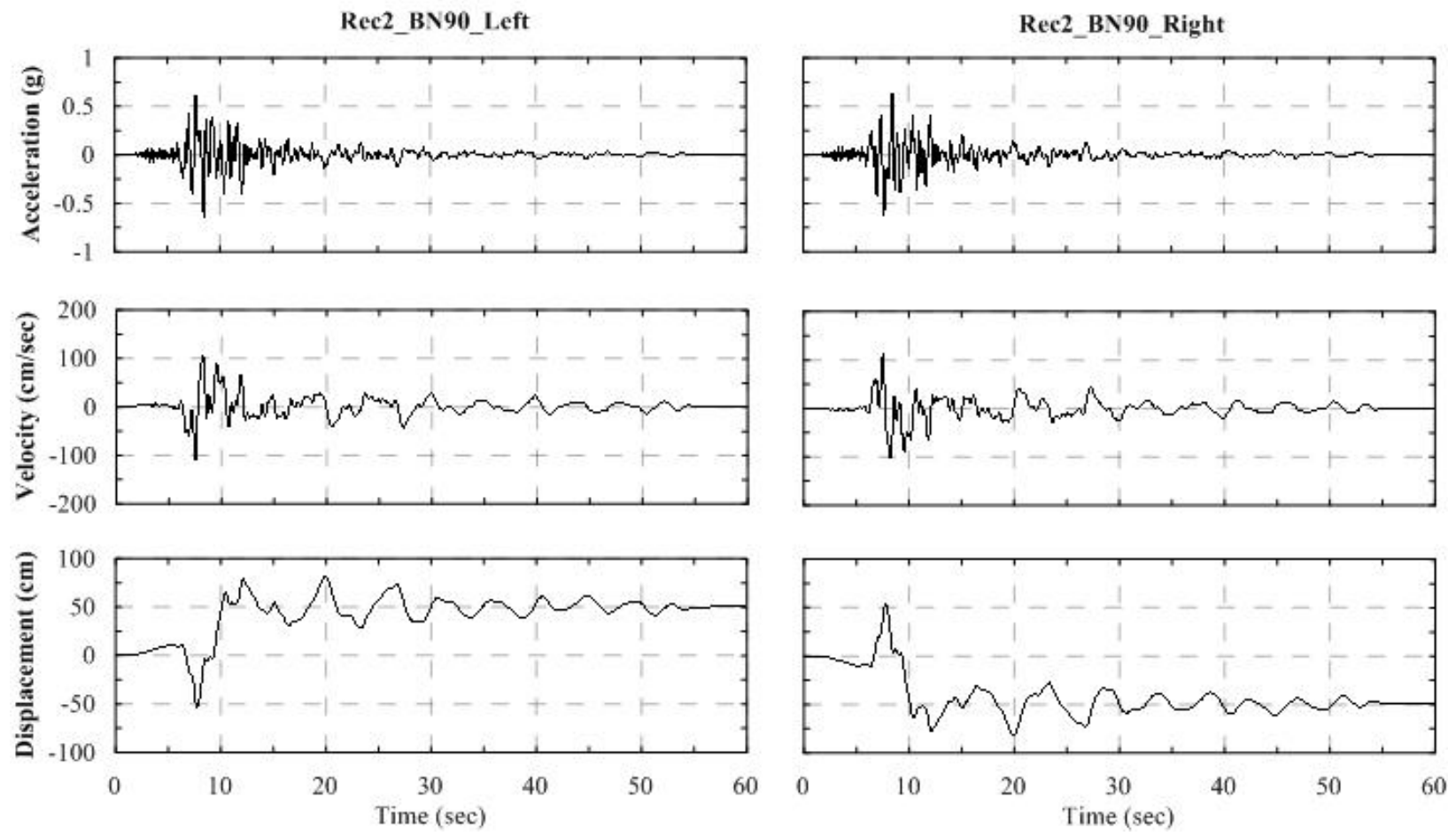

Figure A.5 - Time series summary for Record Set 2 in Bridge-Normal direction
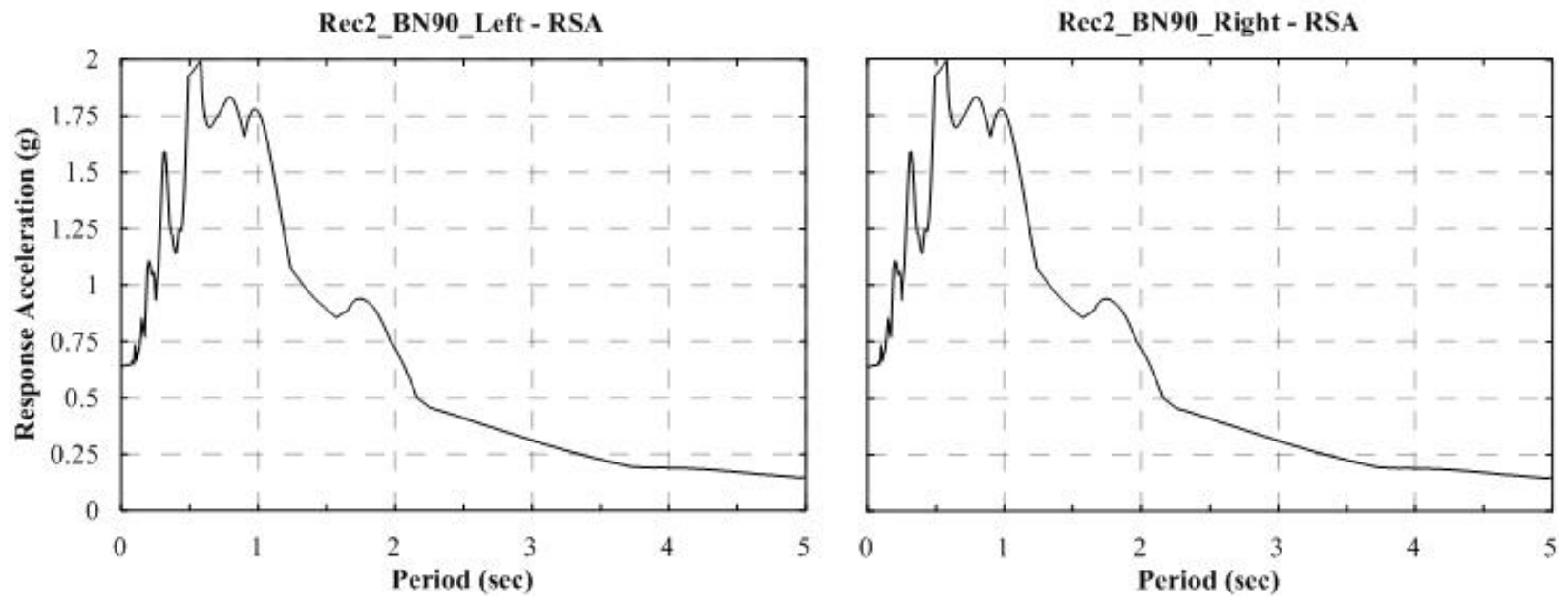

Figure A.6 - Pseudo-Acceleration Response Summary for Record Set 2 in Bridge-Normal direction 

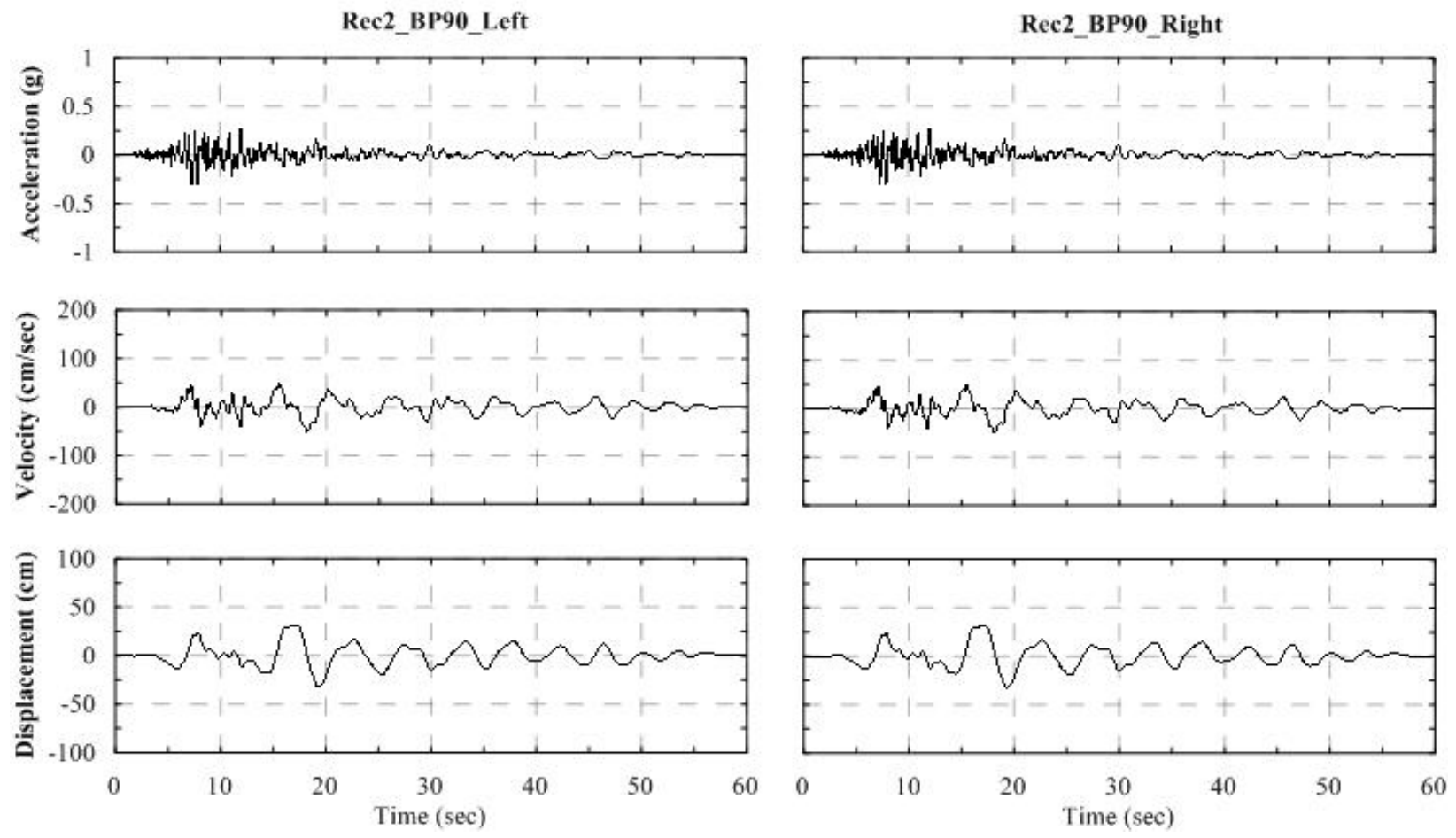

Figure A.7 - Time series summary for Record Set 2 in Bridge-Parallel direction
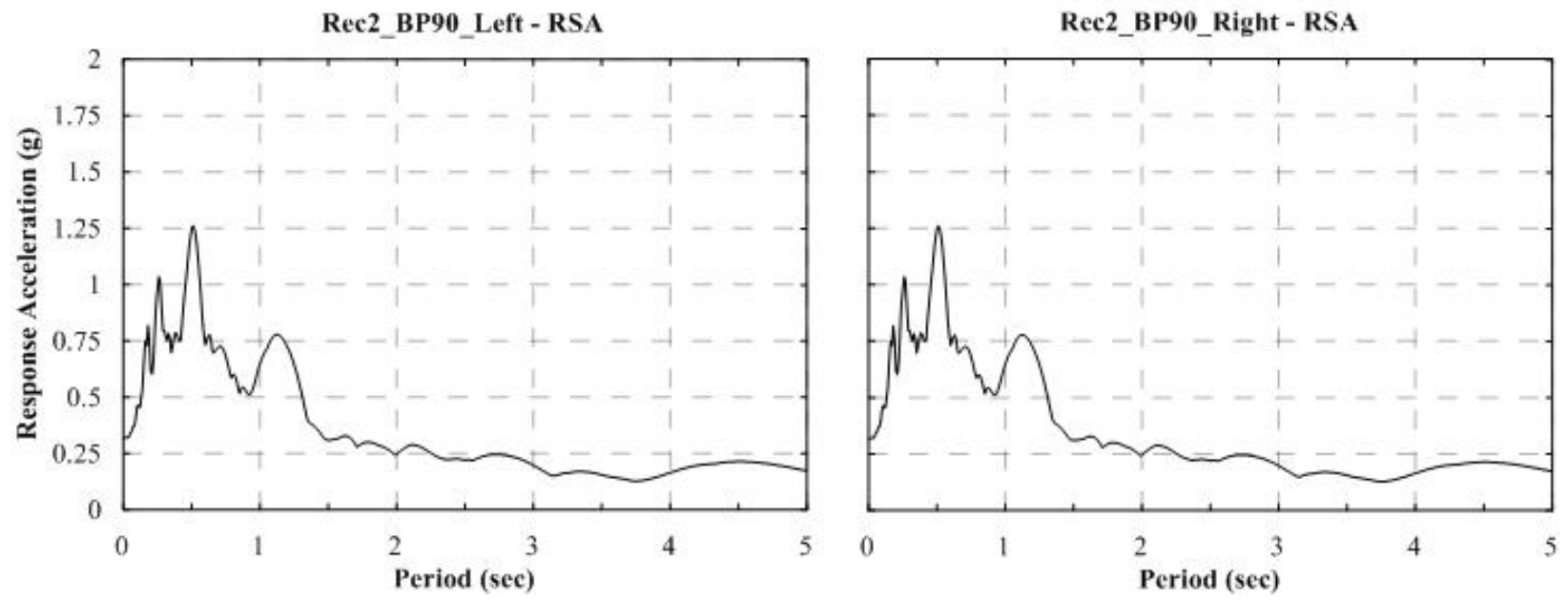

Figure A.8 - Pseudo-Acceleration Response Summary for Record Set 2 in Bridge-Parallel direction 

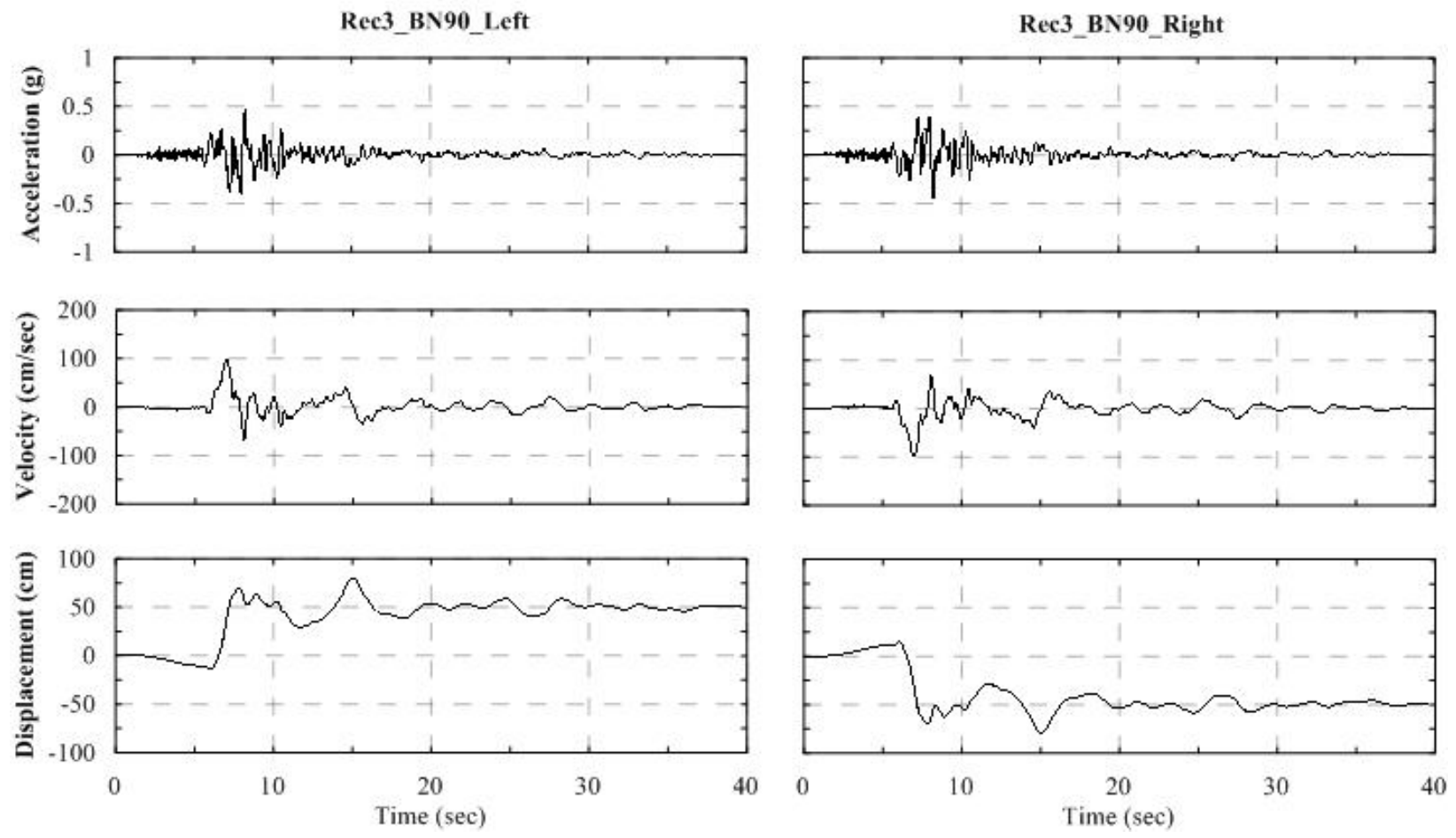

Figure A.9 - Time series summary for Record Set 3 in Bridge-Normal direction
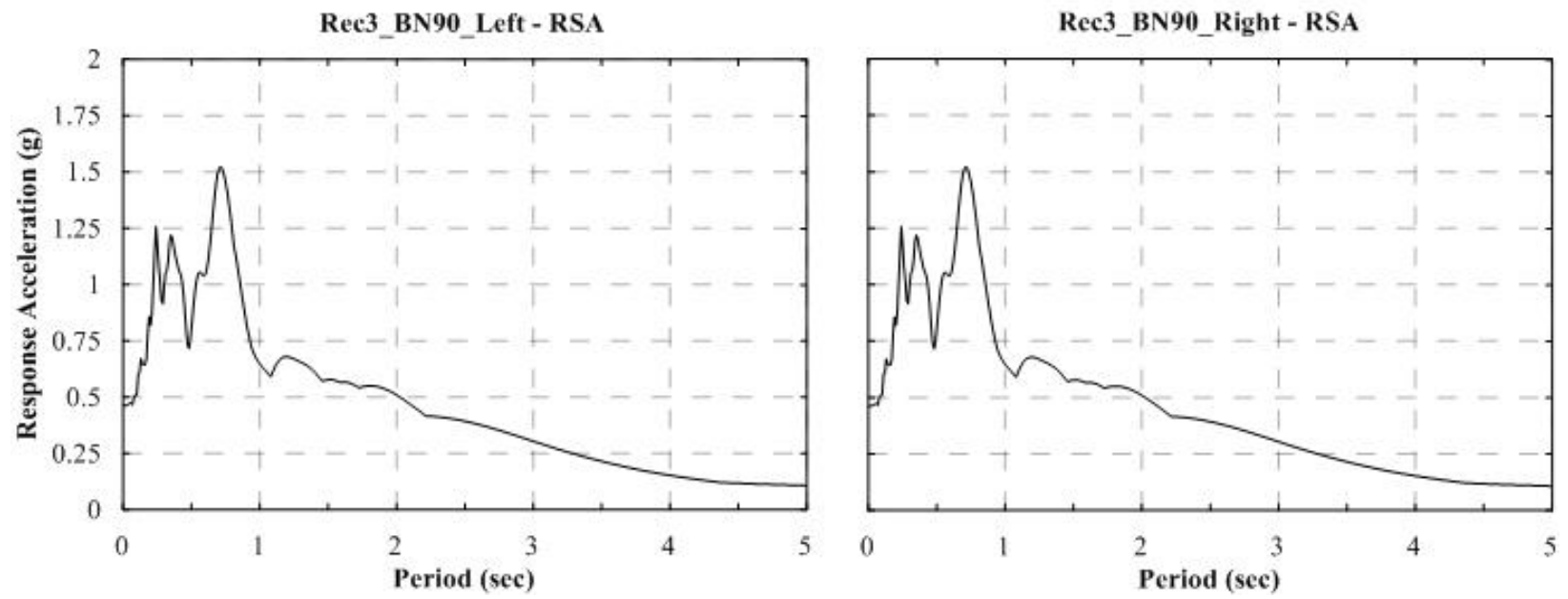

Figure A.10 - Pseudo-Acceleration Response Summary for Record Set 3 in Bridge-Normal direction 

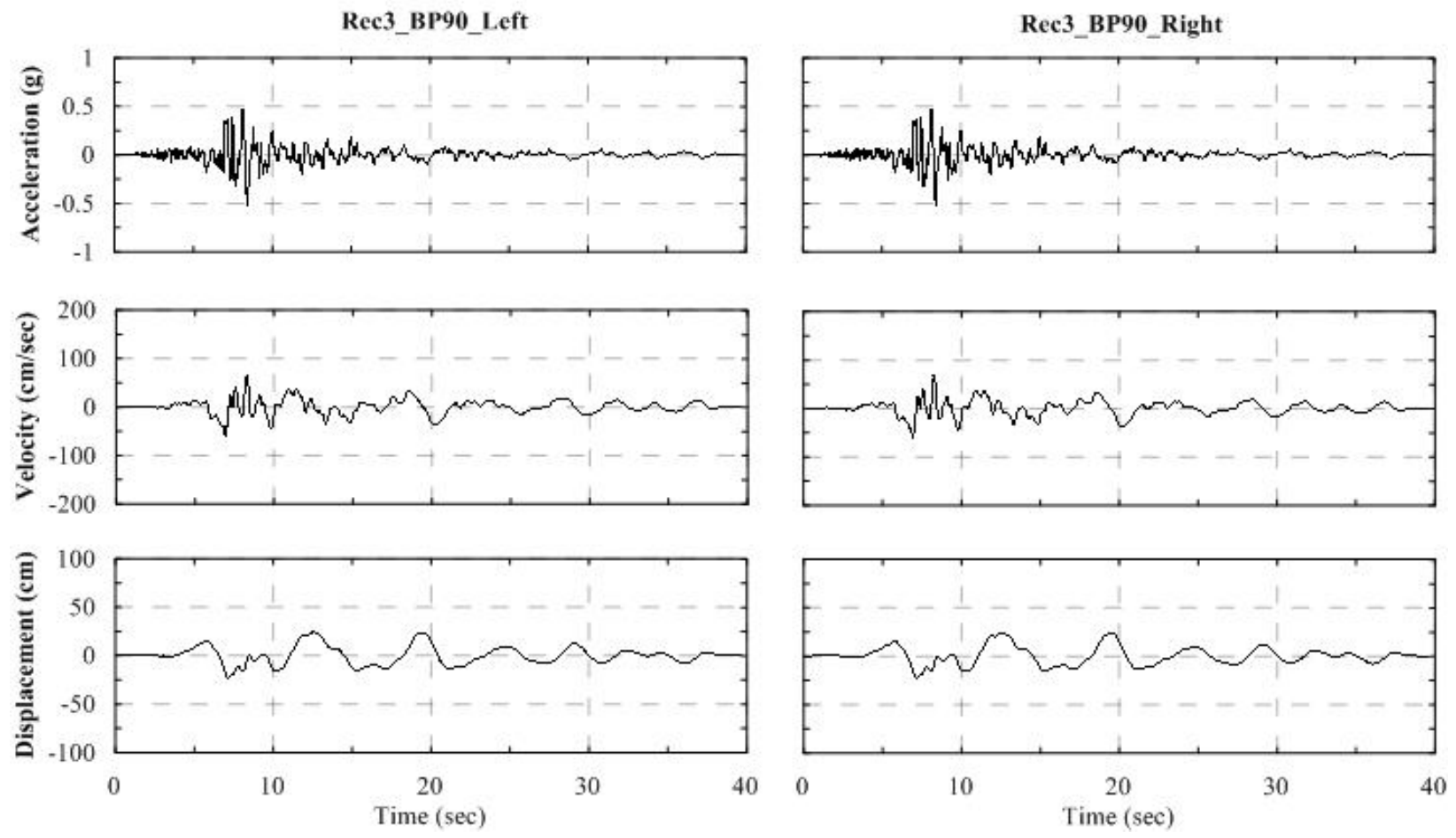

Figure A.11 - Time series summary for Record Set 3 in Bridge-Parallel direction
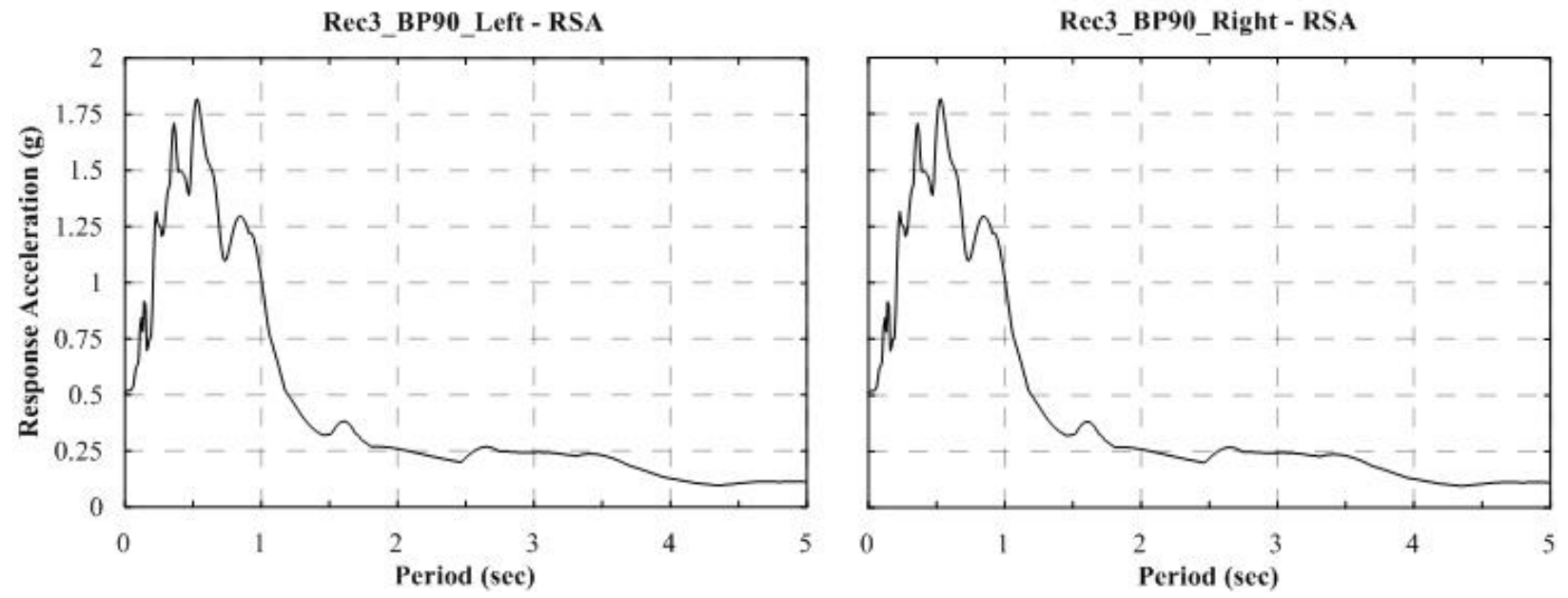

Figure A.12 - Pseudo-Acceleration Response Summary for Record Set 3 in Bridge-Parallel direction 

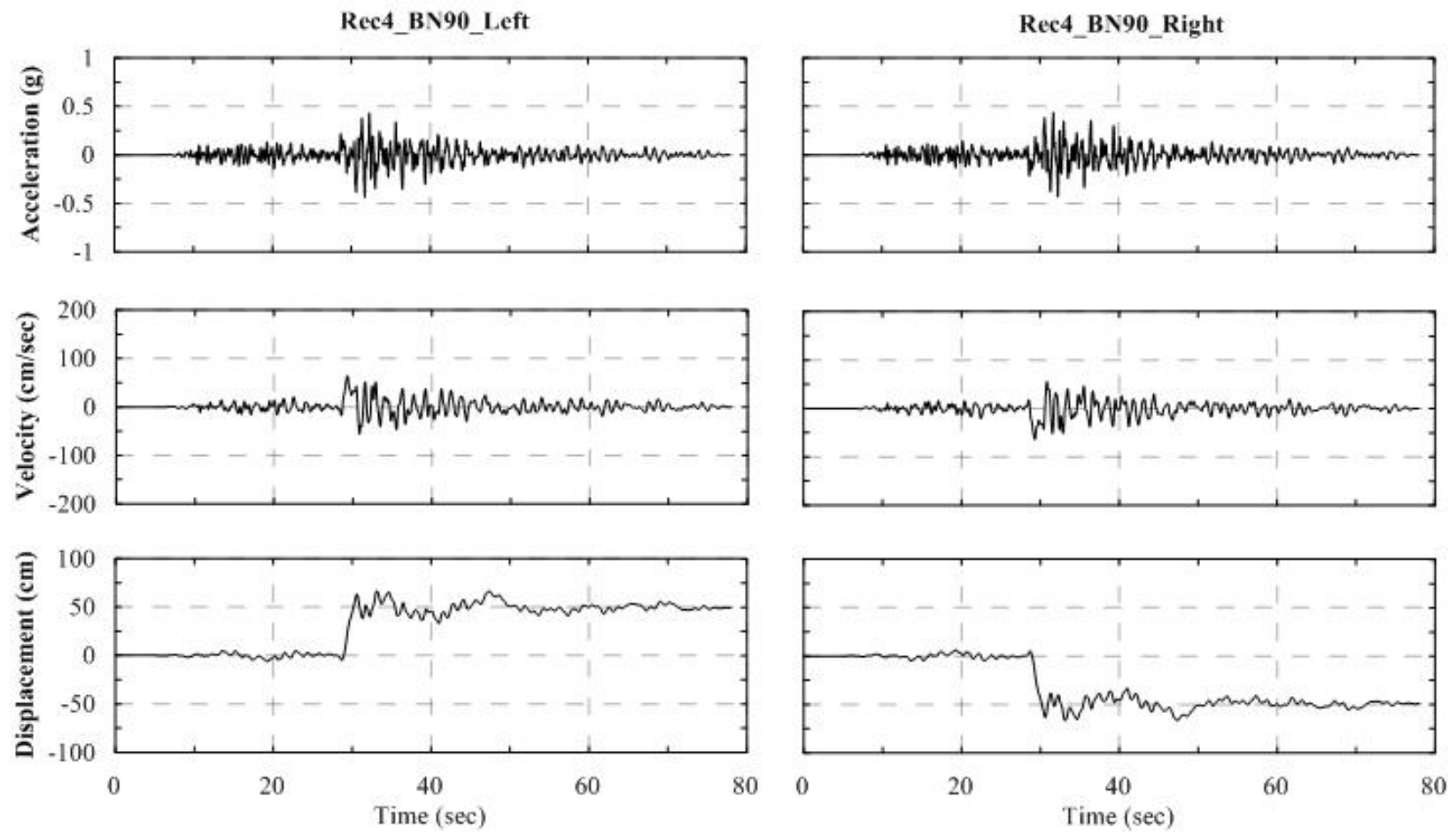

Figure A.13 - Time series summary for Record Set 4 in Bridge-Normal direction
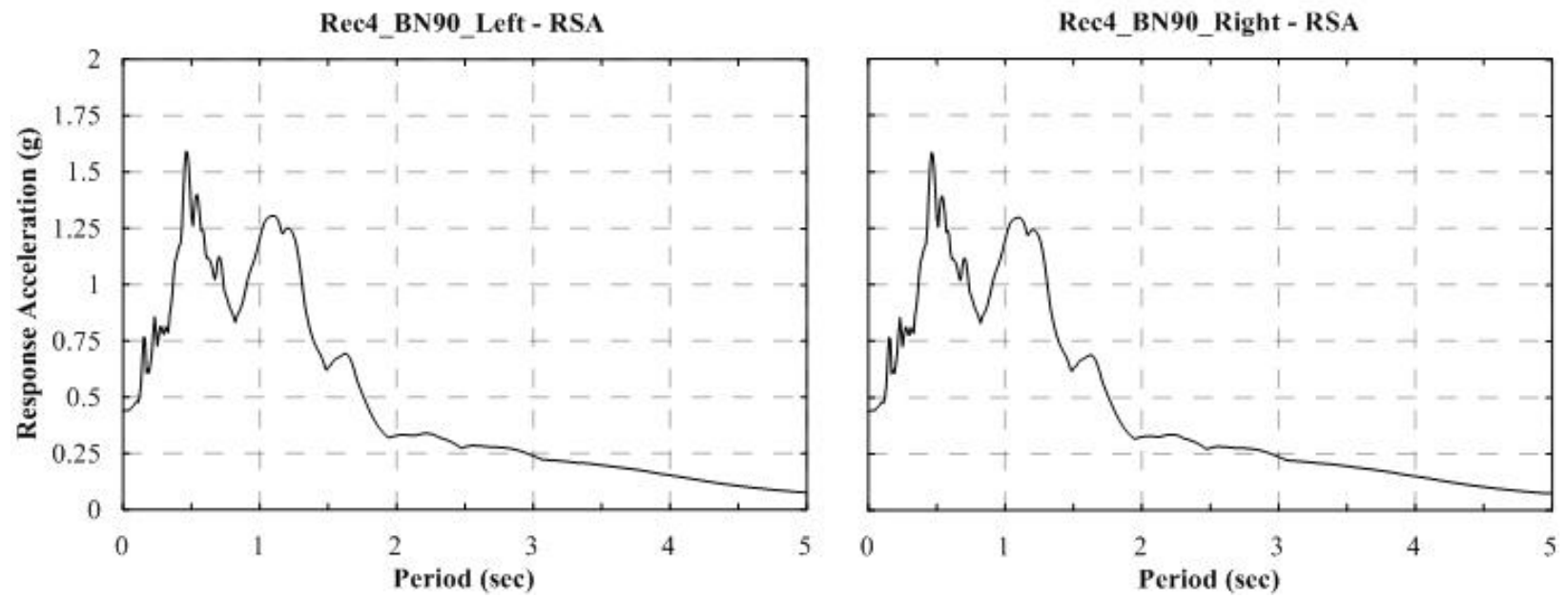

Figure A.14 - Pseudo-Acceleration Response Summary for Record Set 4 in Bridge-Normal direction 

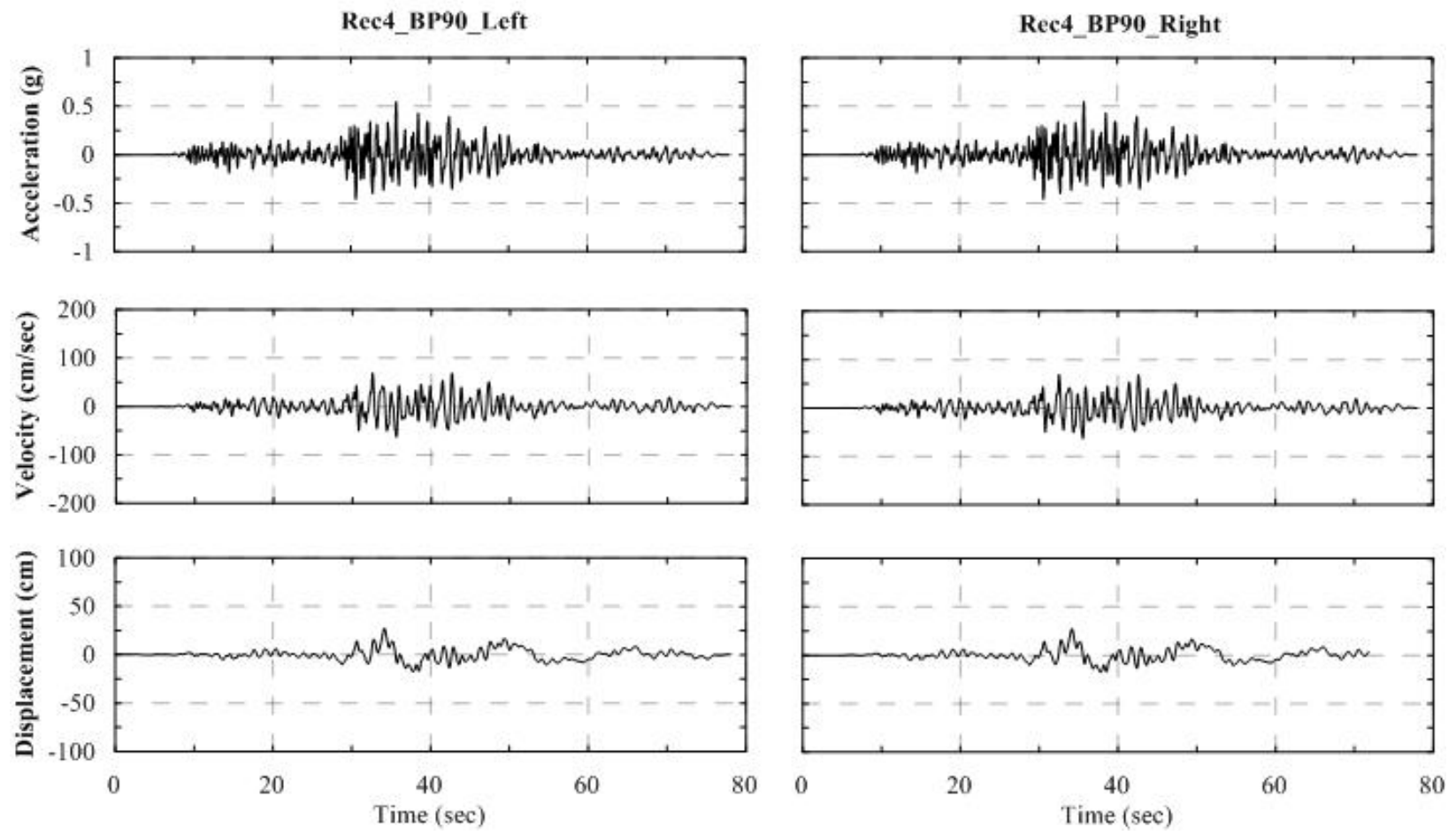

Figure A.15 - Time series summary for Record Set 4 in Bridge-Parallel direction
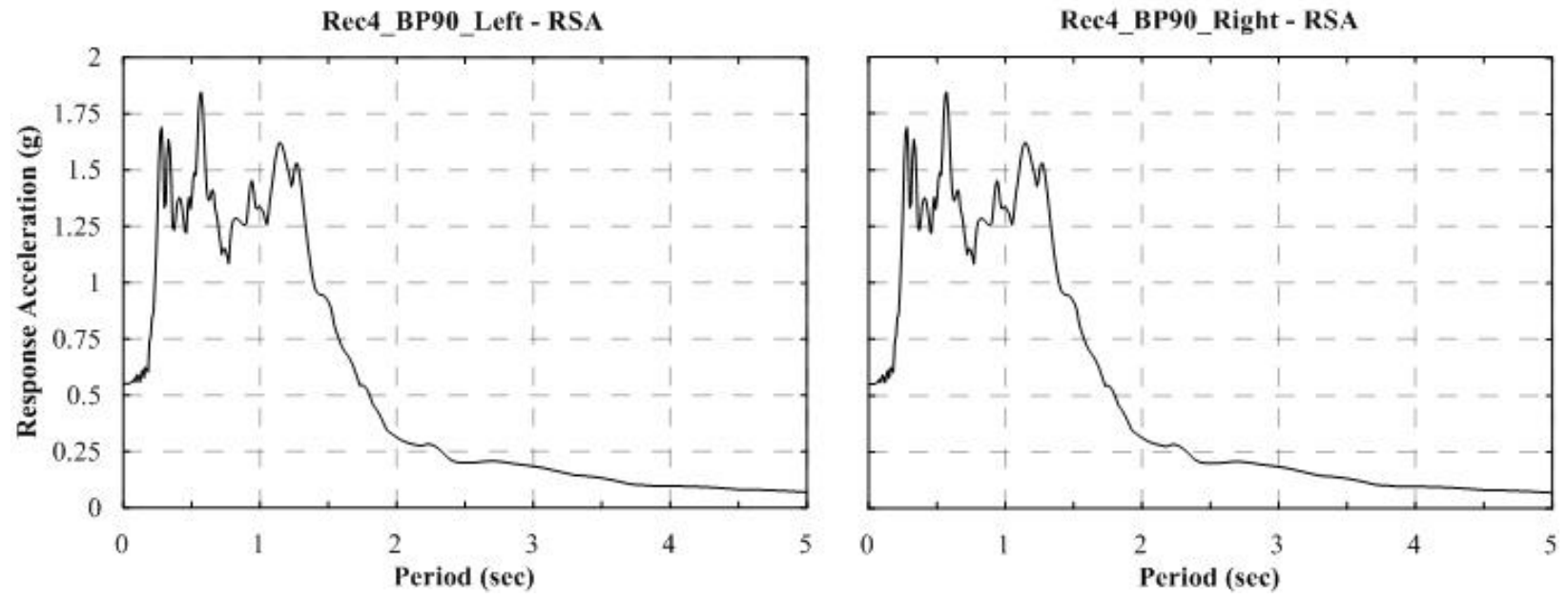

Figure A.16 - Pseudo-Acceleration Response Summary for Record Set 4 in Bridge-Parallel direction 

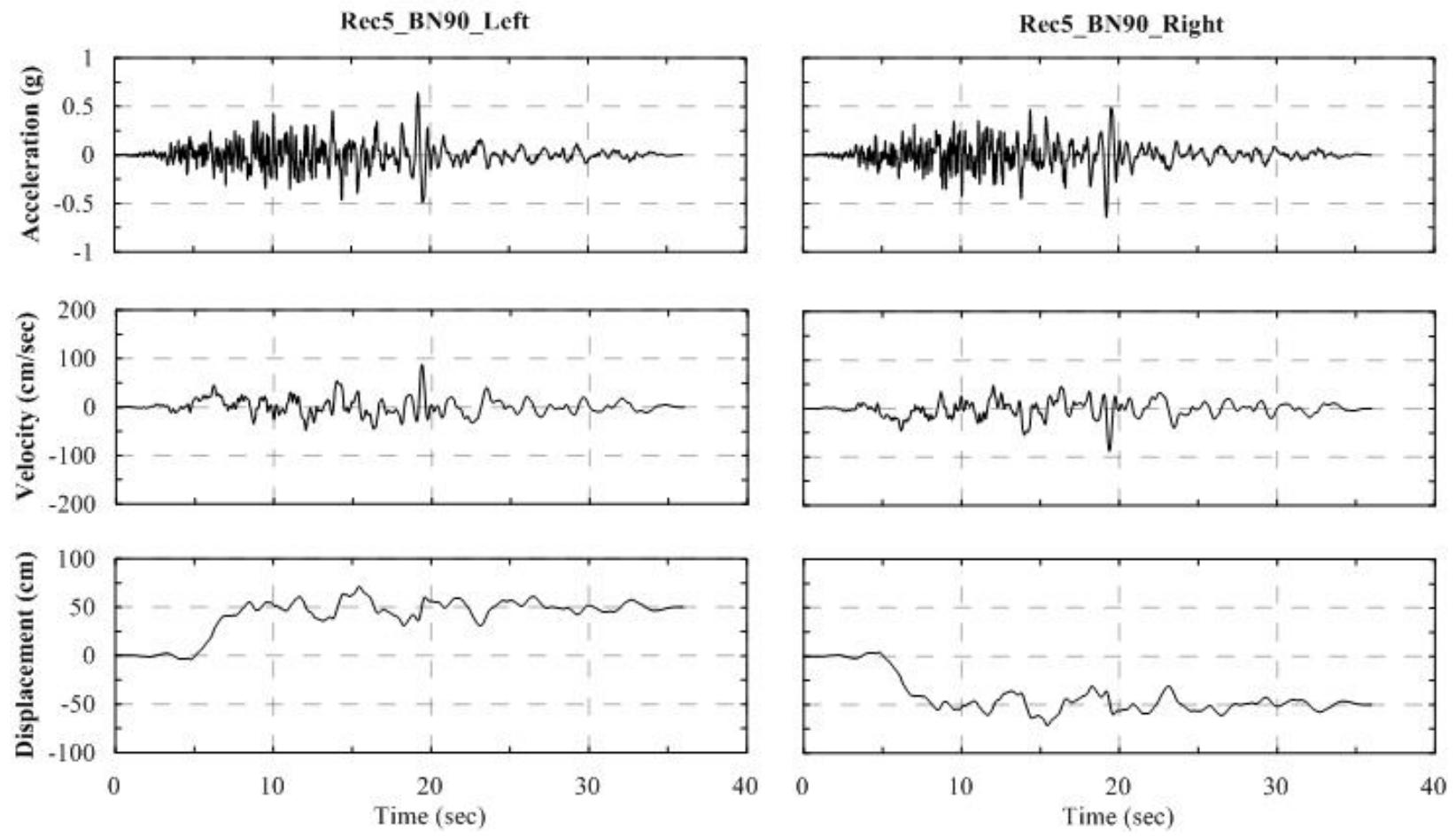

Figure A.17 - Time series summary for Record Set 5 in Bridge-Normal direction
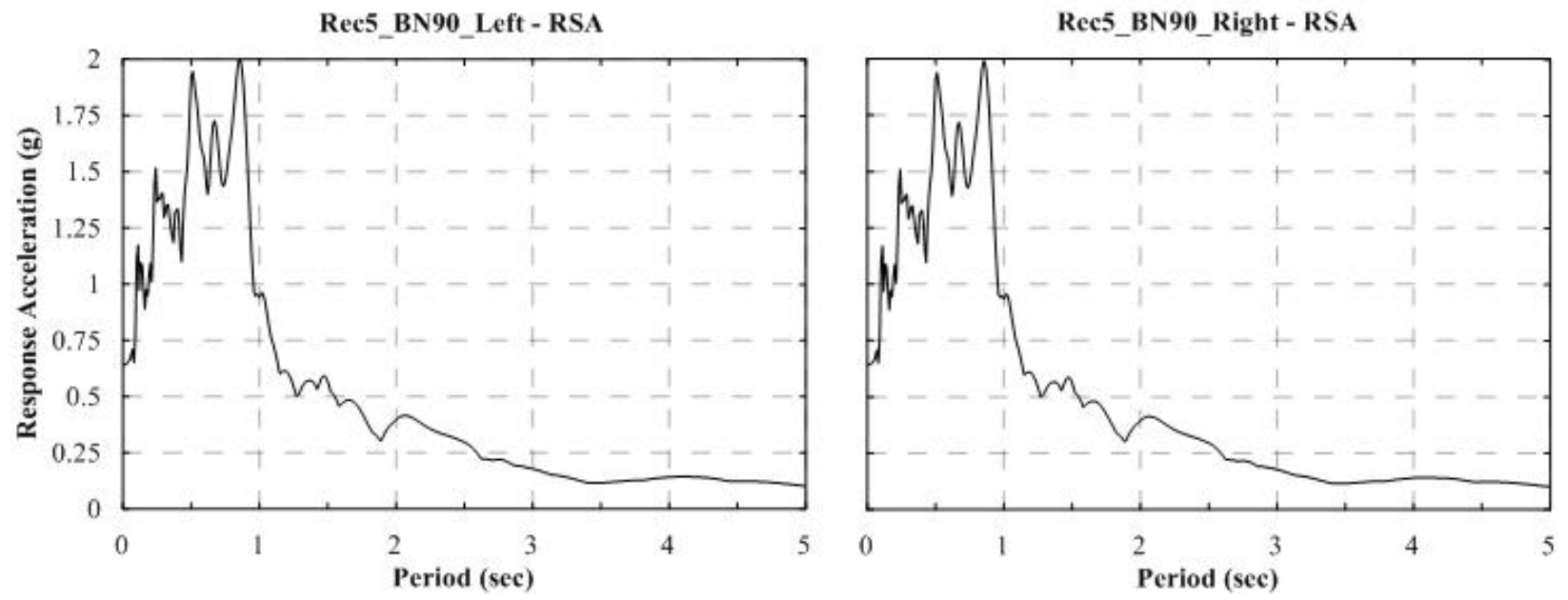

Figure A.18 - Pseudo-Acceleration Response Summary for Record Set 5 in Bridge-Normal direction 

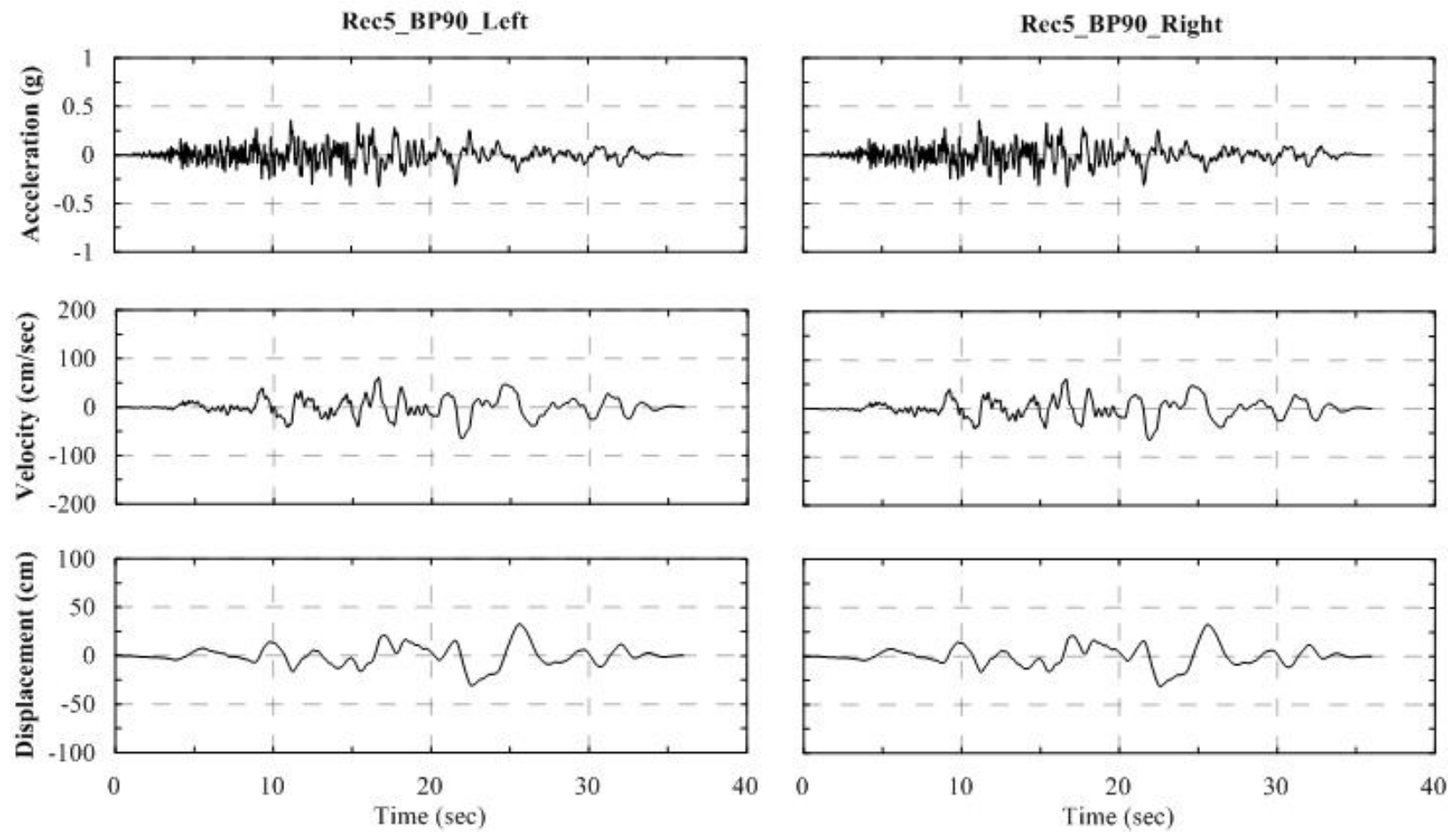

Figure A.19 - Time series summary for Record Set 5 in Bridge-Parallel direction
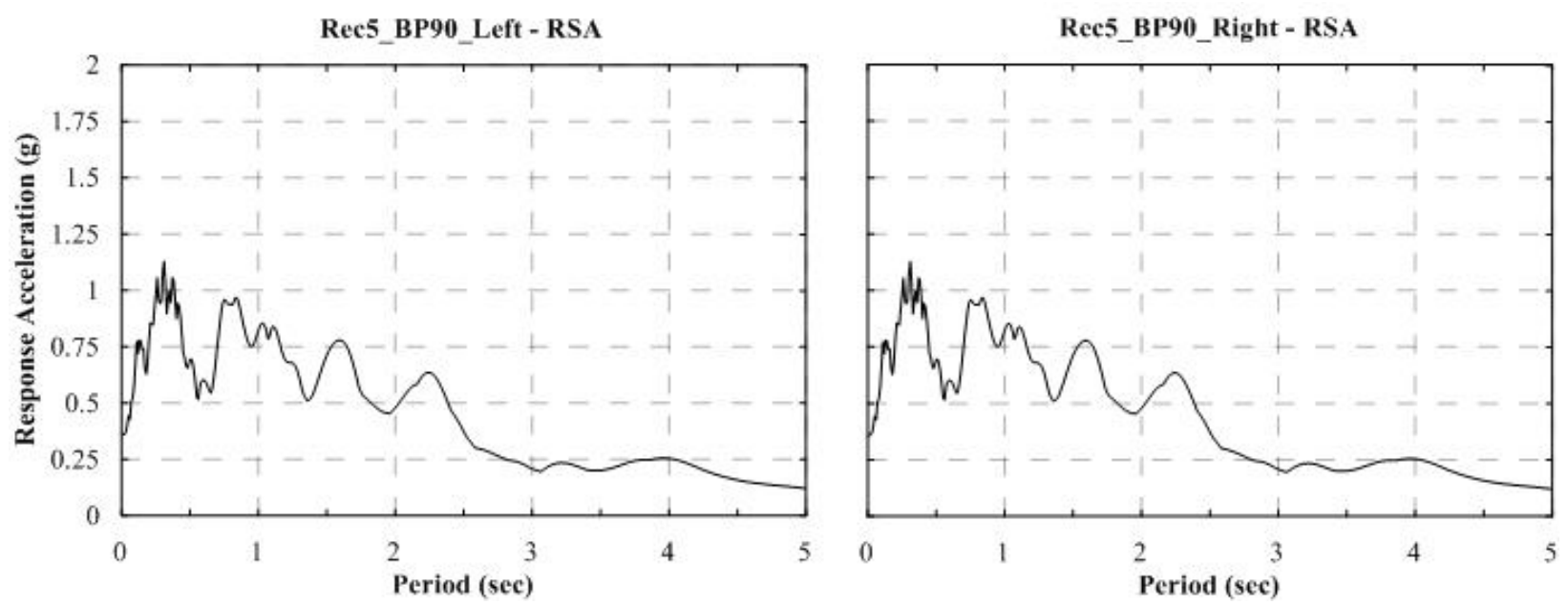

Figure A.20 - Pseudo-Acceleration Response Summary for Record Set 5 in Bridge-Parallel direction 

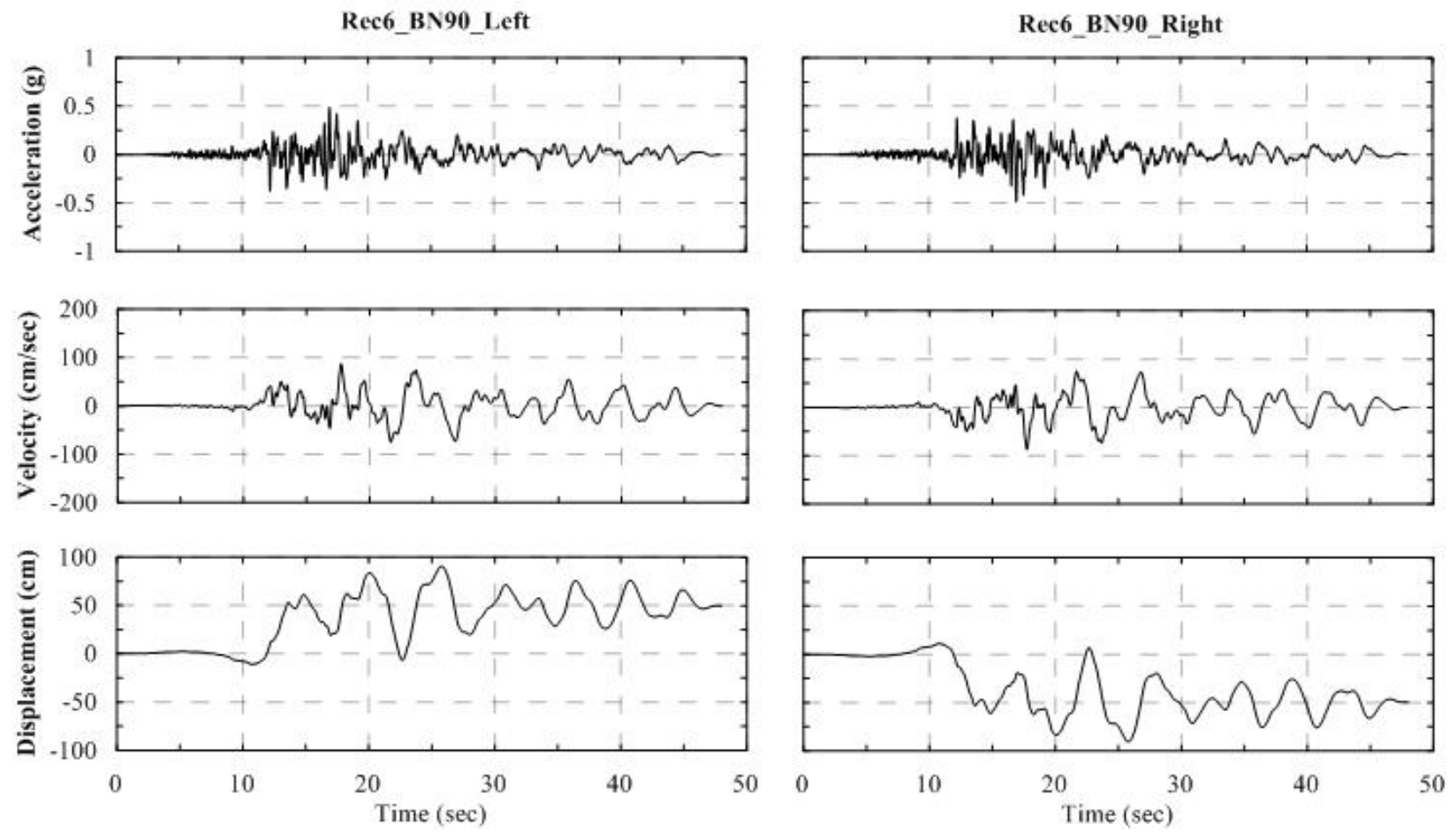

Figure A.21 - Time series summary for Record Set 6 in Bridge-Normal direction
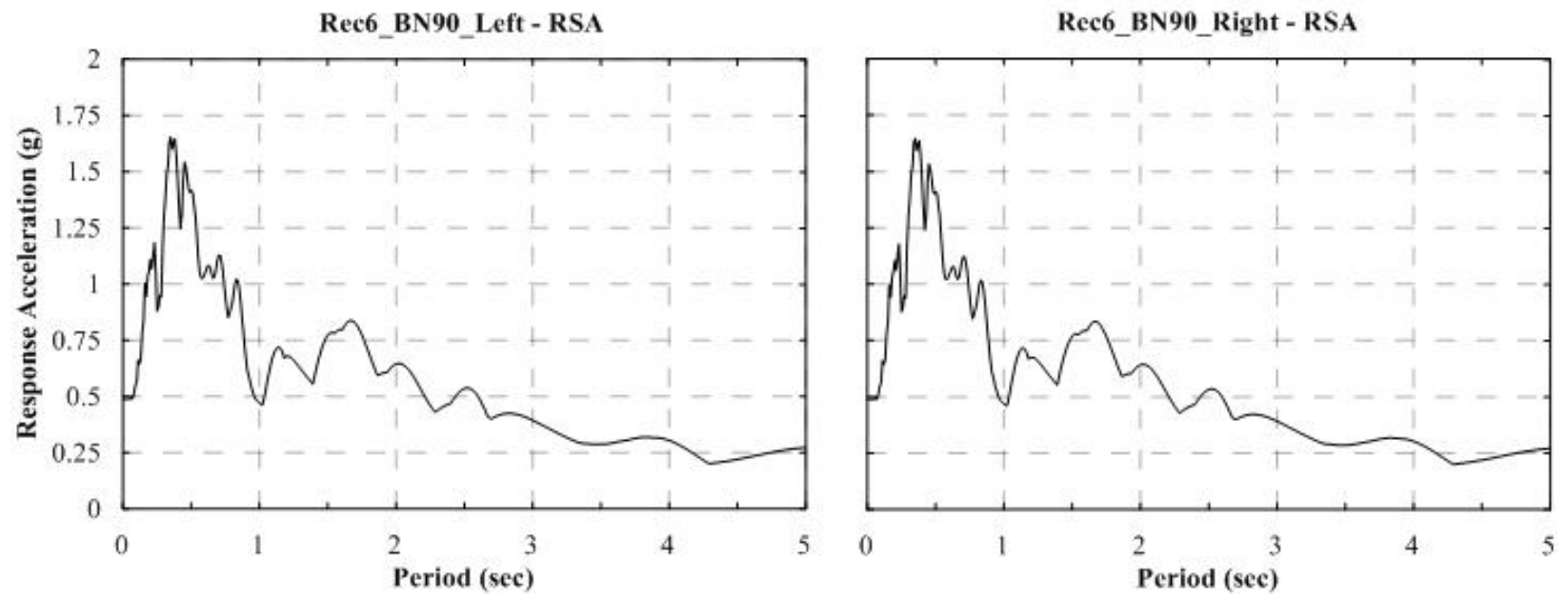

Figure A.22 - Pseudo-Acceleration Response Summary for Record Set 6 in Bridge-Normal direction 

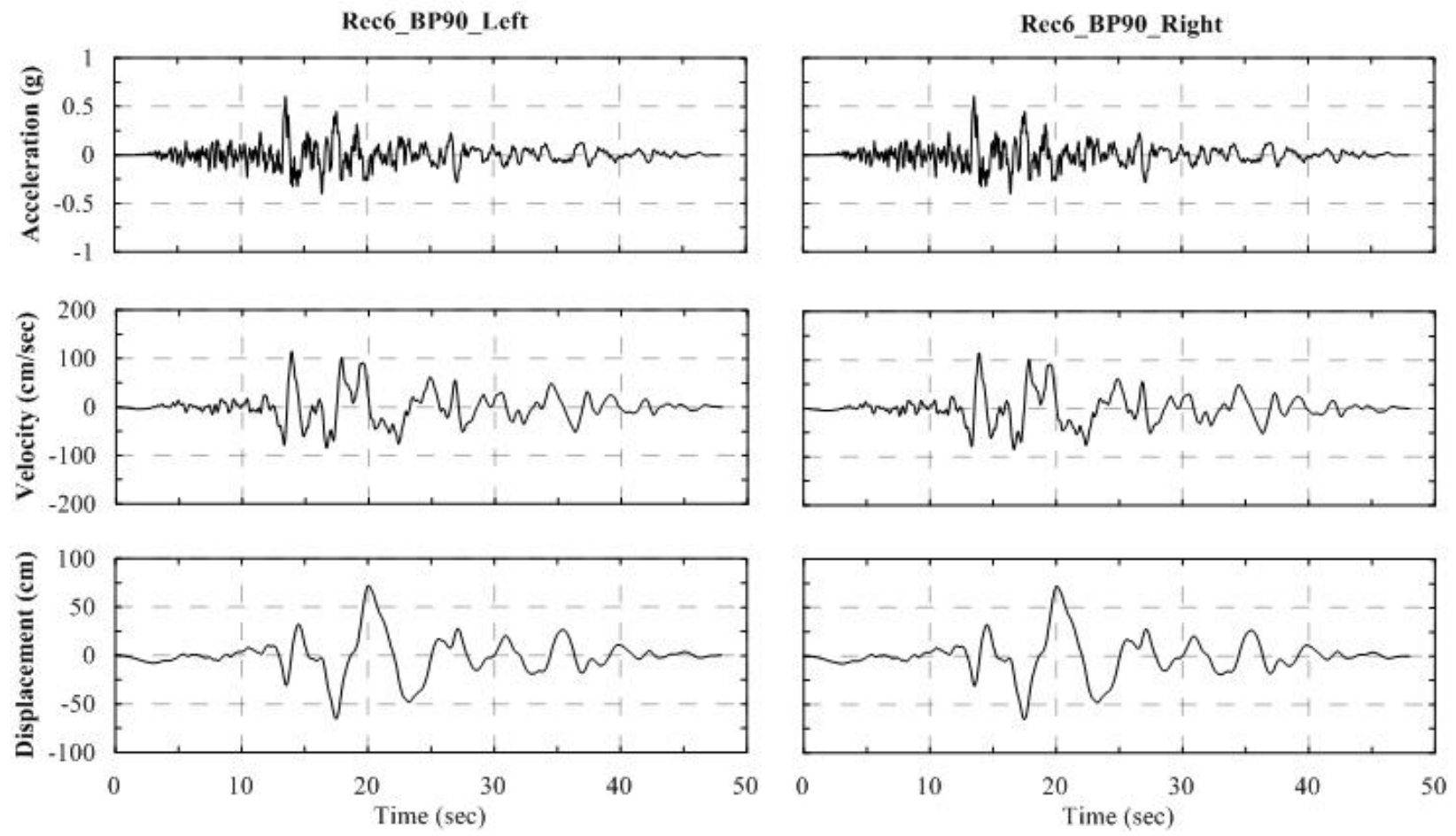

Figure A.23 - Time series summary for Record Set 6 in Bridge-Parallel direction
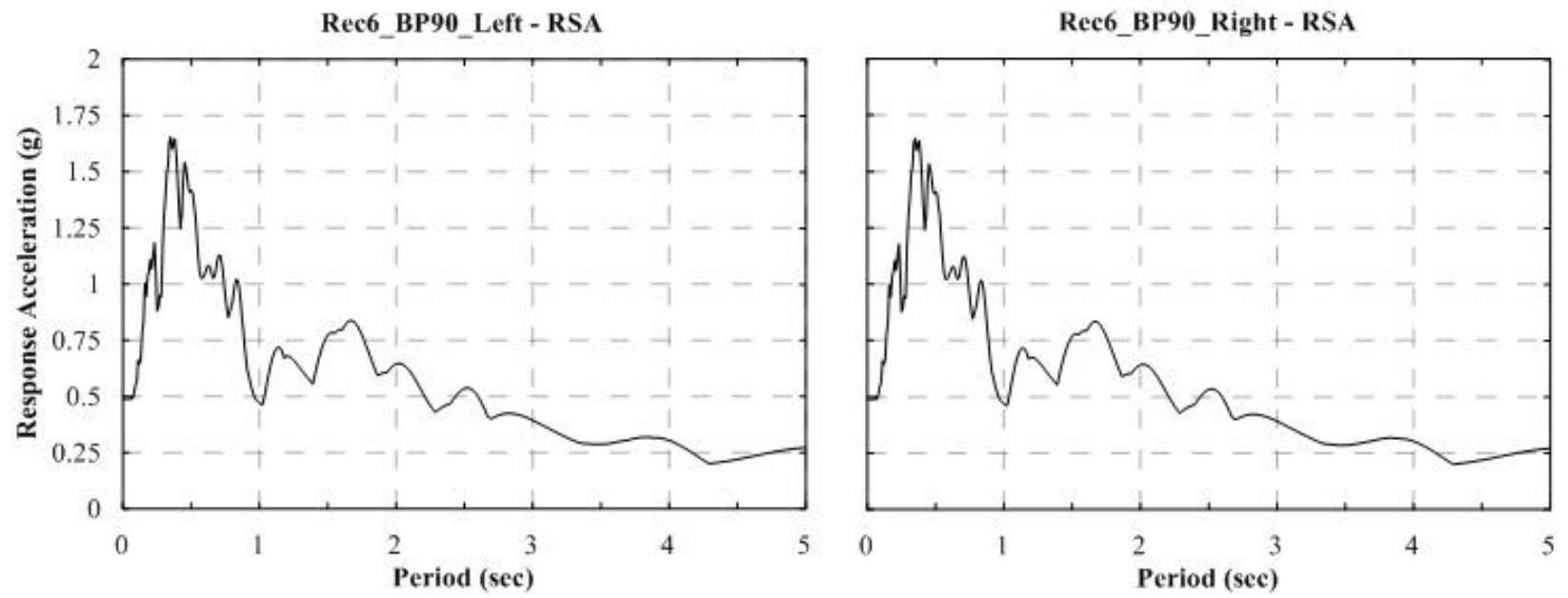

Figure A.24 - Pseudo-Acceleration Response Summary for Record Set 6 in Bridge-Parallel direction 

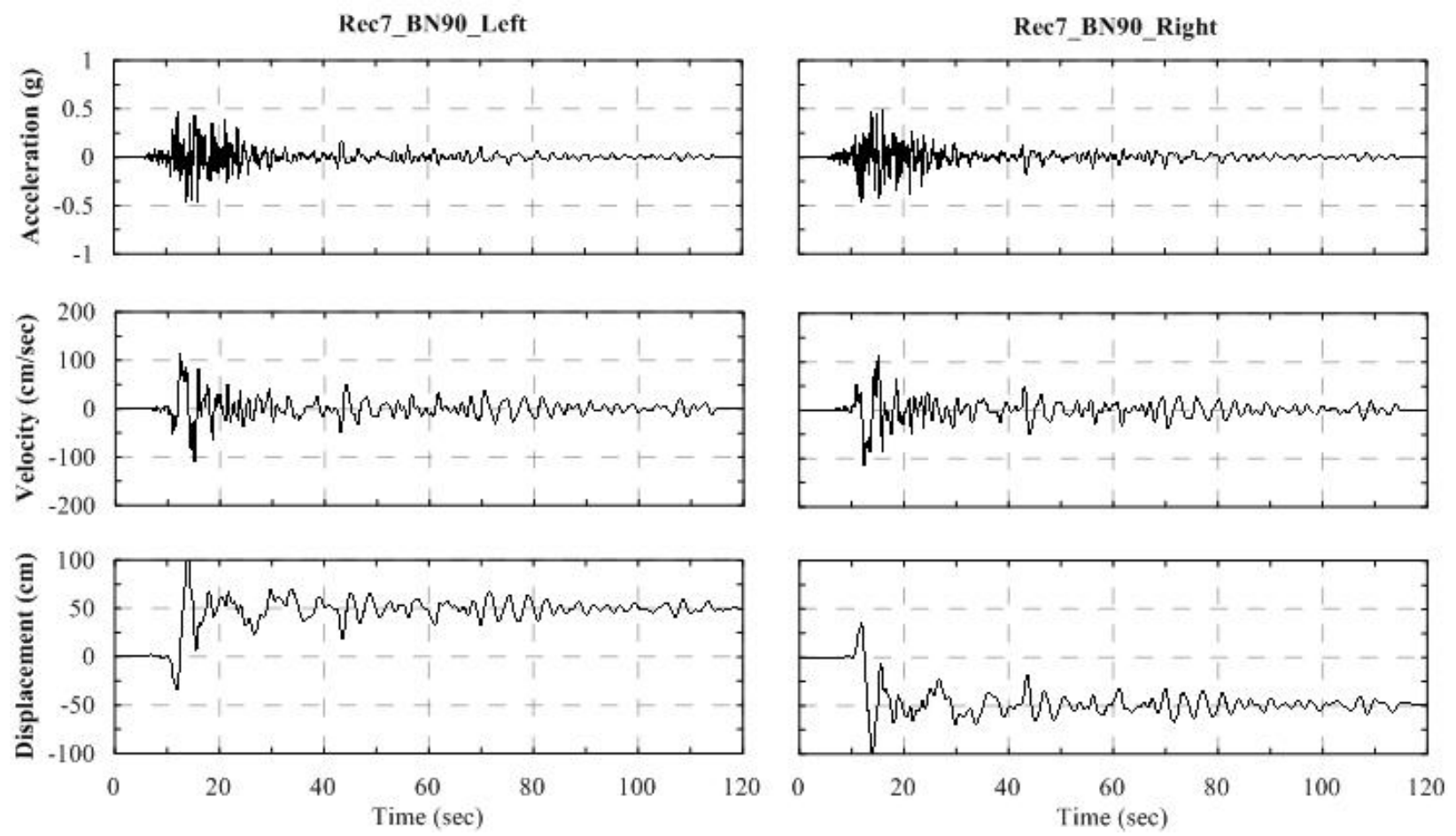

Figure A.25 - Time series summary for Record Set 7 in Bridge-Normal direction
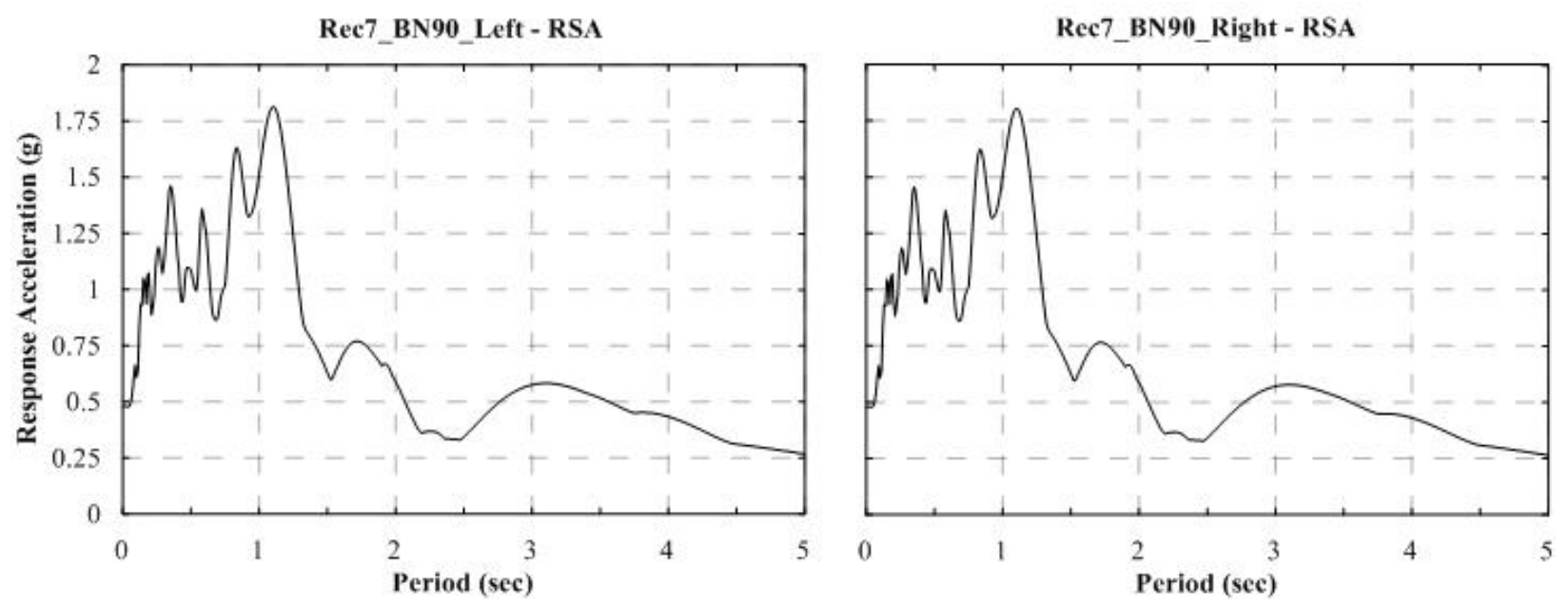

Figure A.26 - Pseudo-Acceleration Response Summary for Record Set 7 in Bridge-Normal direction 

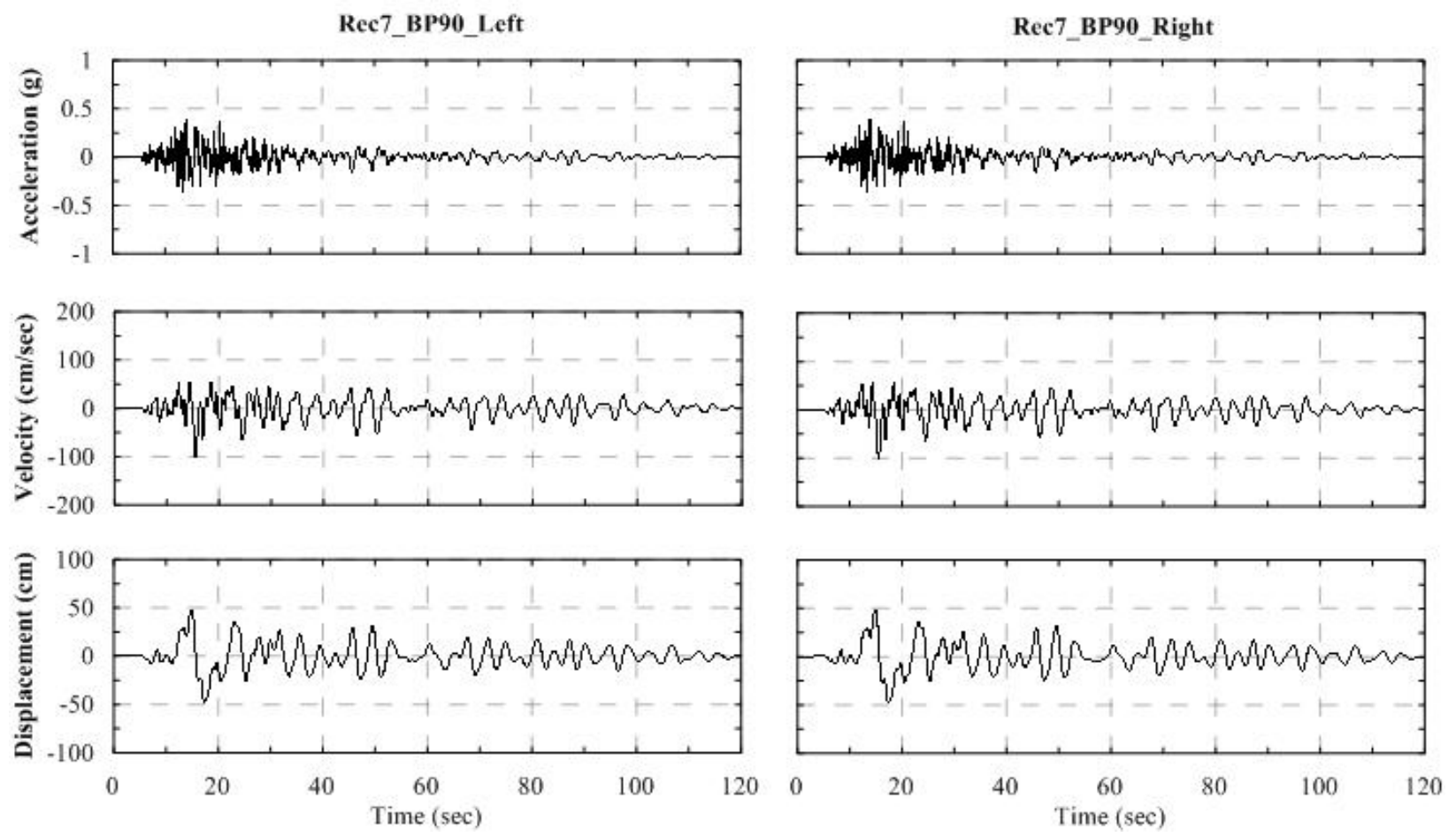

Figure A.27 - Time series summary for Record Set 7 in Bridge-Parallel direction
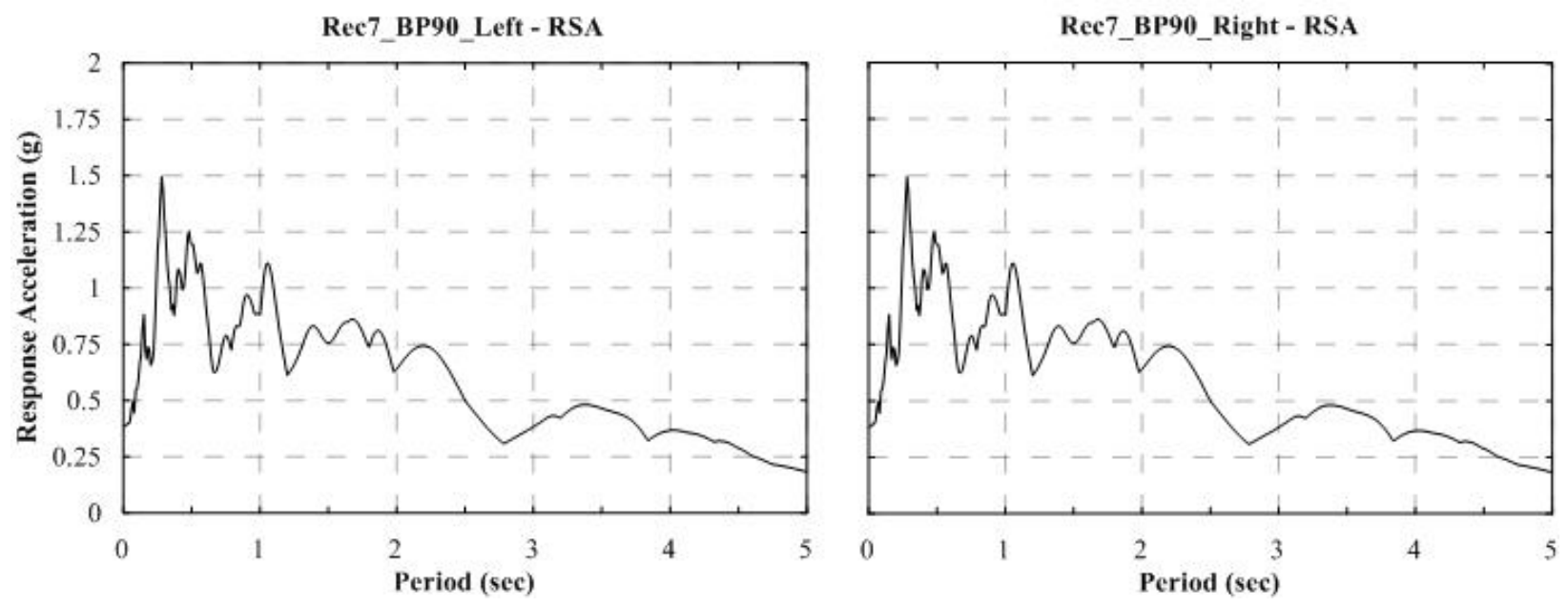

Figure A.28 - Pseudo-Acceleration Response Summary for Record Set 7 in Bridge-Parallel direction 

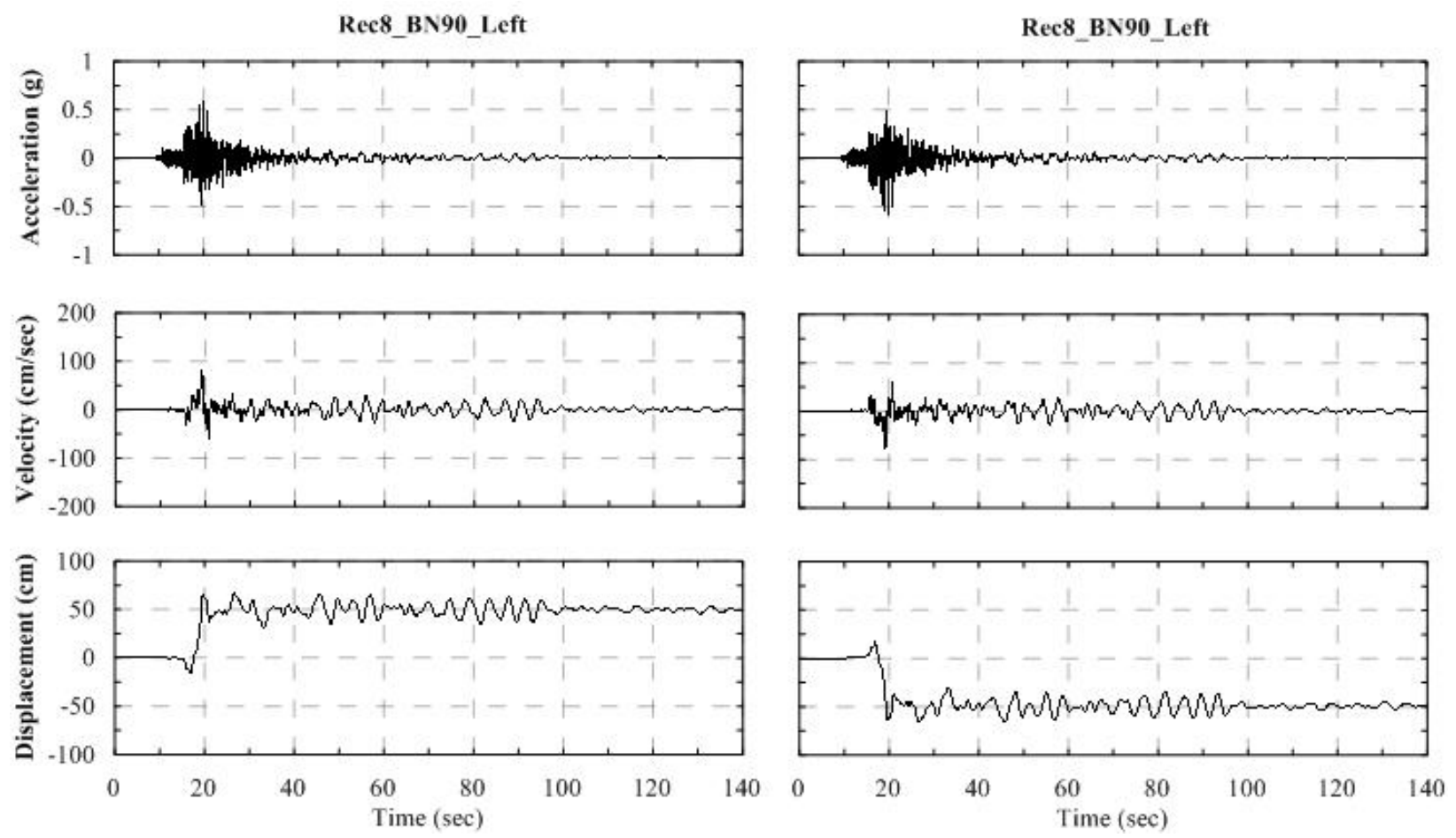

Figure A.29 - Time series summary for Record Set 8 in Bridge-Normal direction
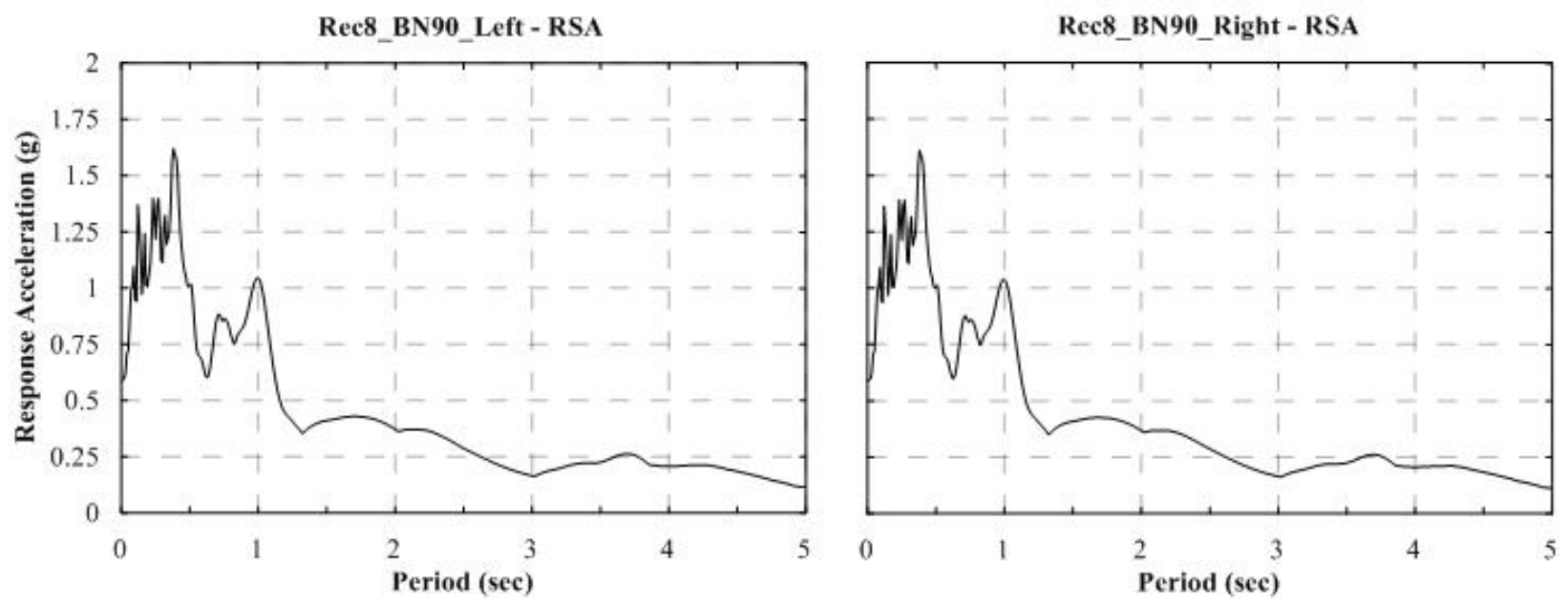

Figure A.30 - Pseudo-Acceleration Response Summary for Record Set 8 in Bridge-Normal direction 

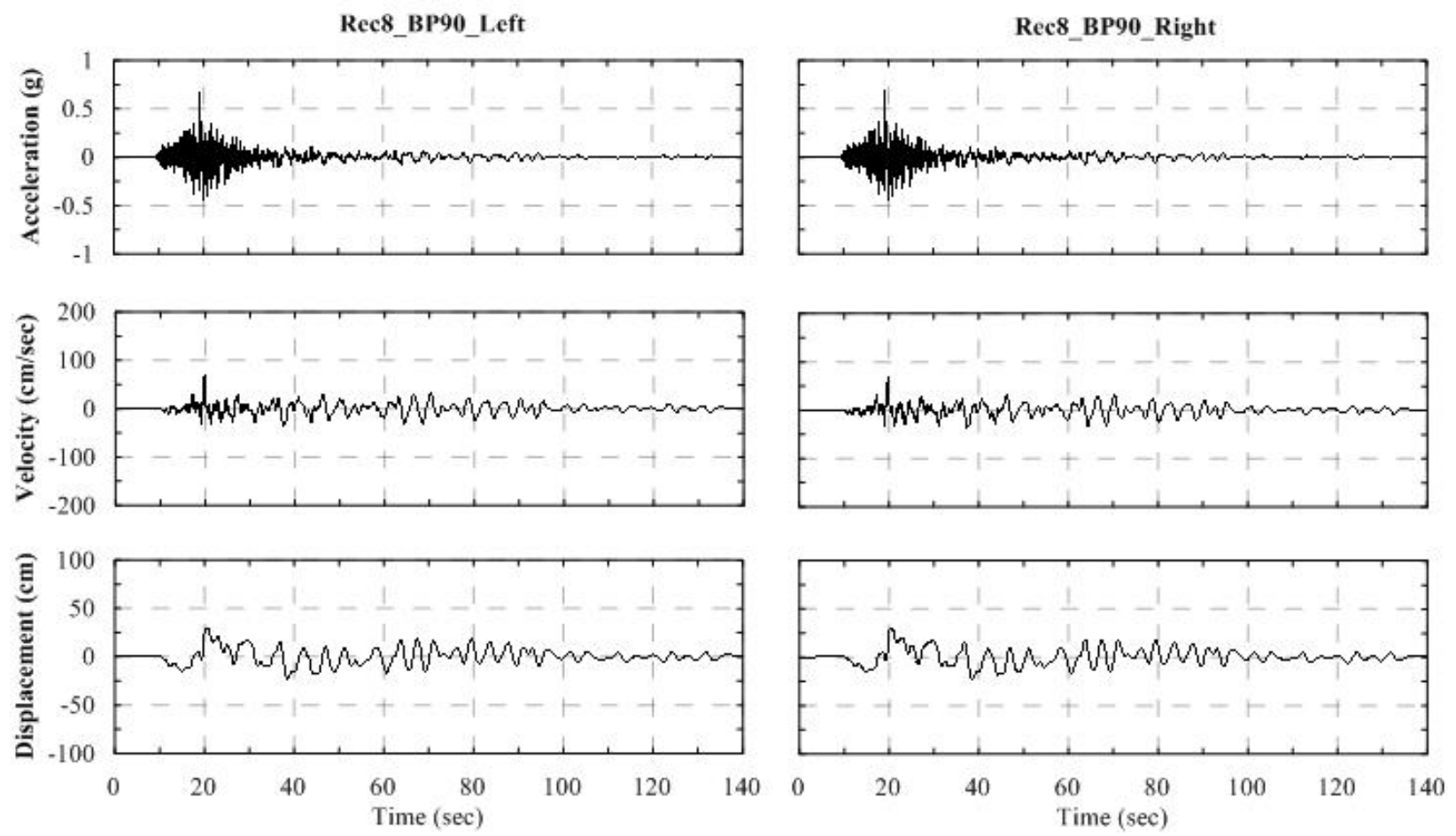

Figure A.31 - Time series summary for Record Set 8 in Bridge-Parallel direction
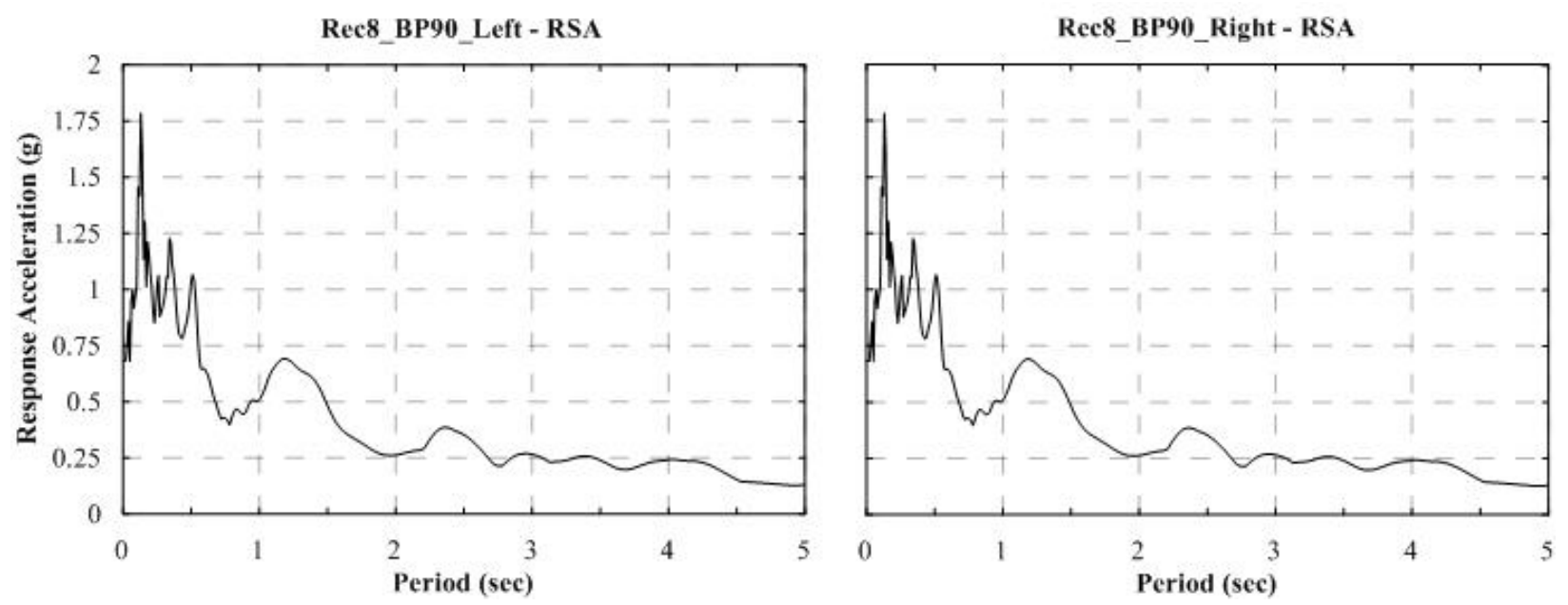

Figure A.32 - Pseudo-Acceleration Response Summary for Record Set 8 in Bridge-Parallel direction 

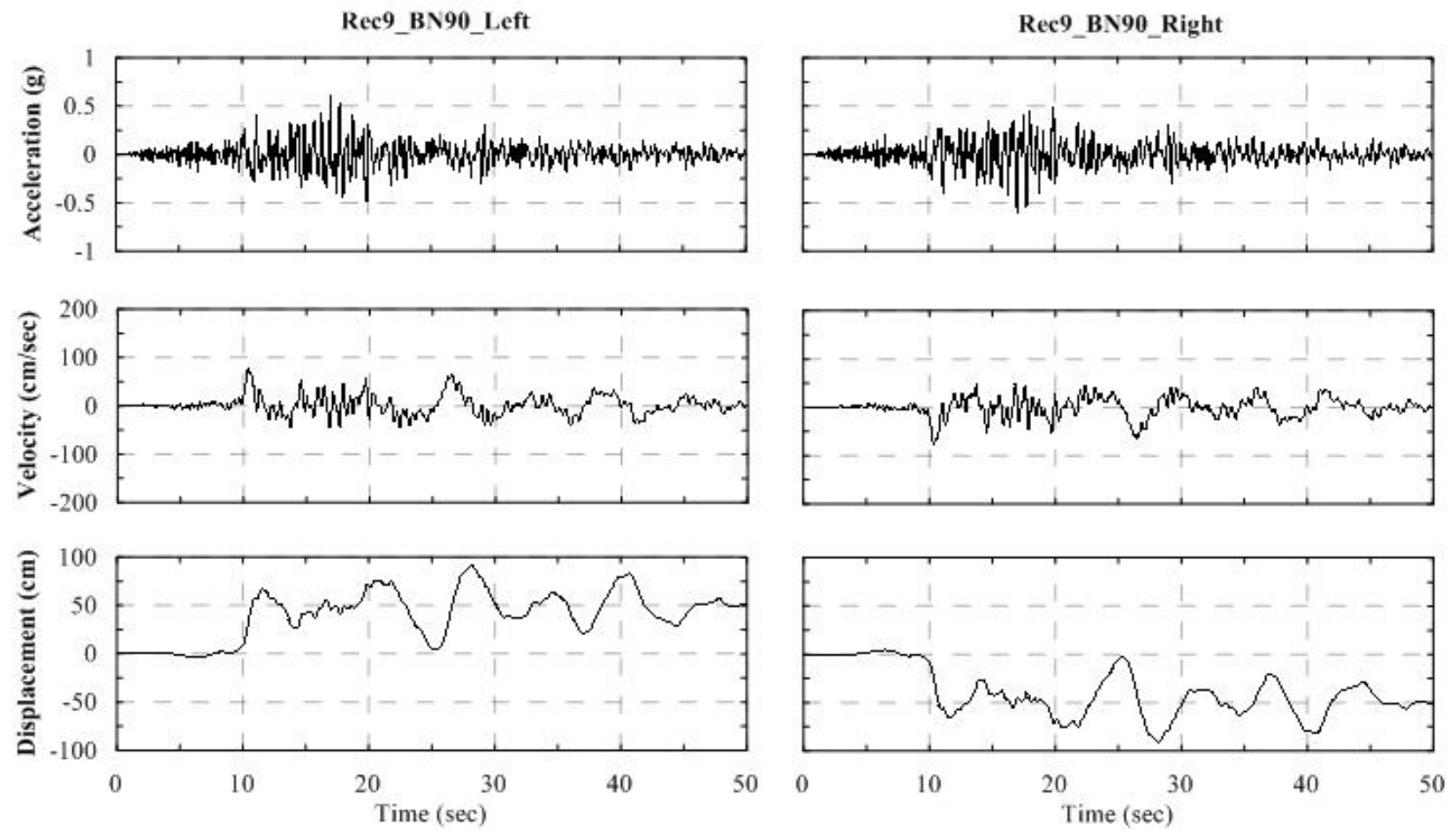

Figure A.33 - Time series summary for Record Set 9 in Bridge-Normal direction
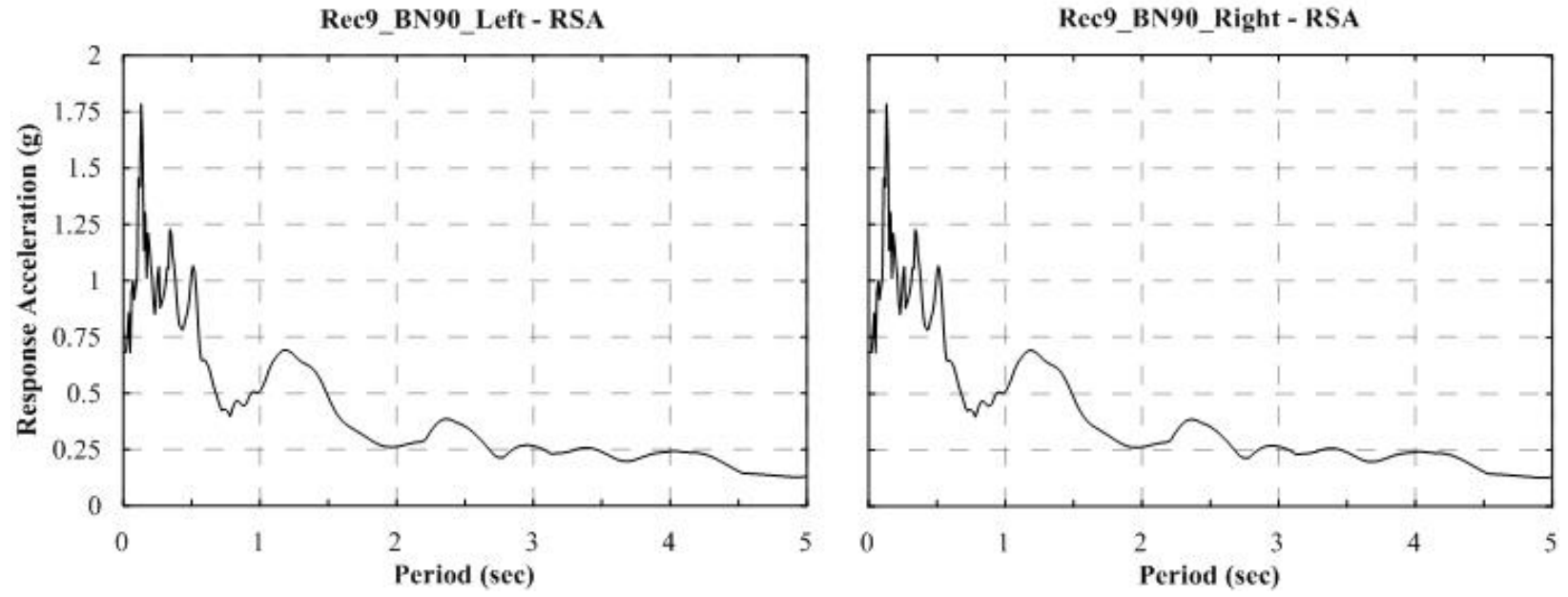

Figure A.34 - Pseudo-Acceleration Response Summary for Record Set 9 in Bridge-Normal direction 

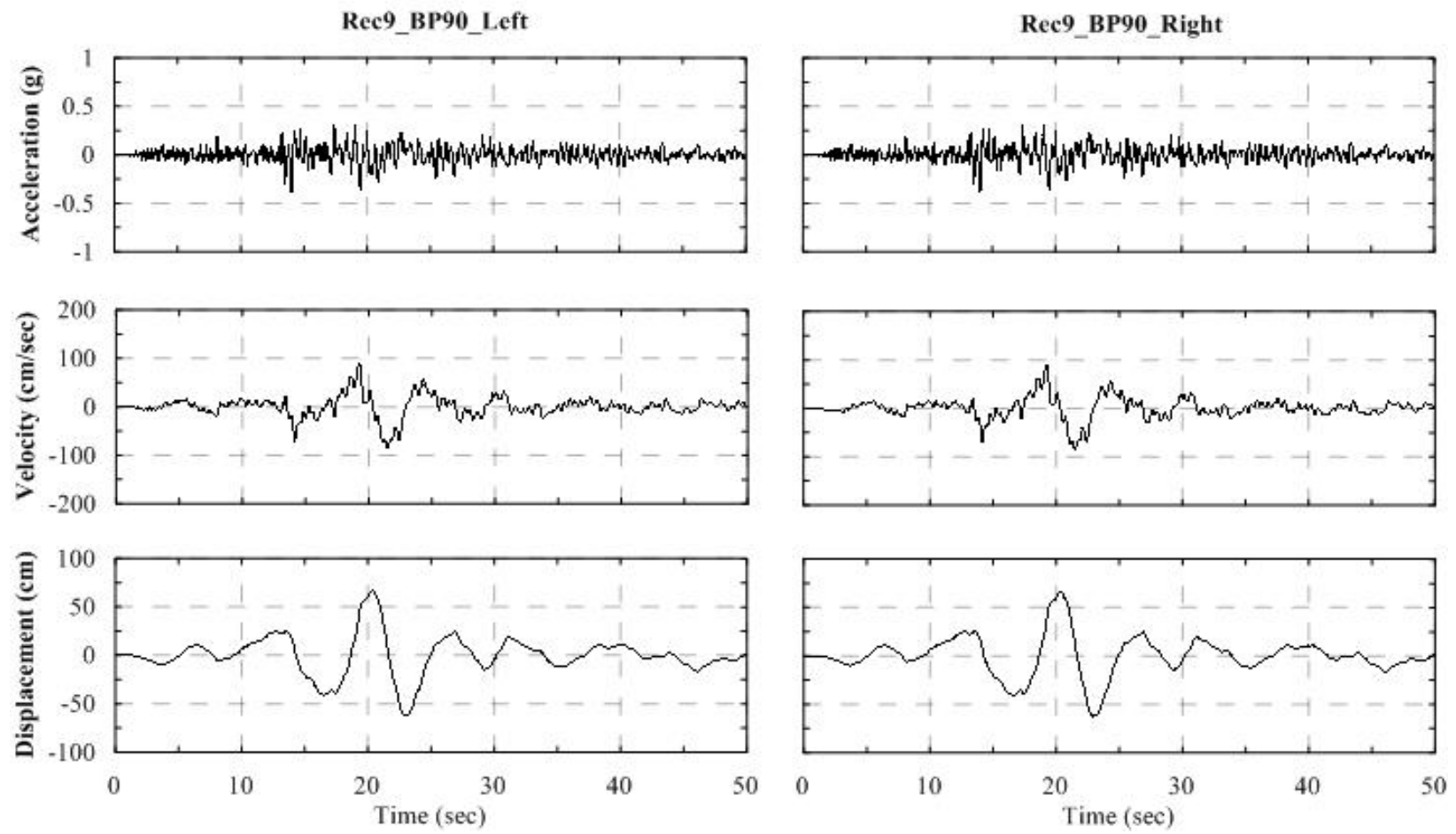

Figure A.35 - Time series summary for Record Set 9 in Bridge-Parallel direction
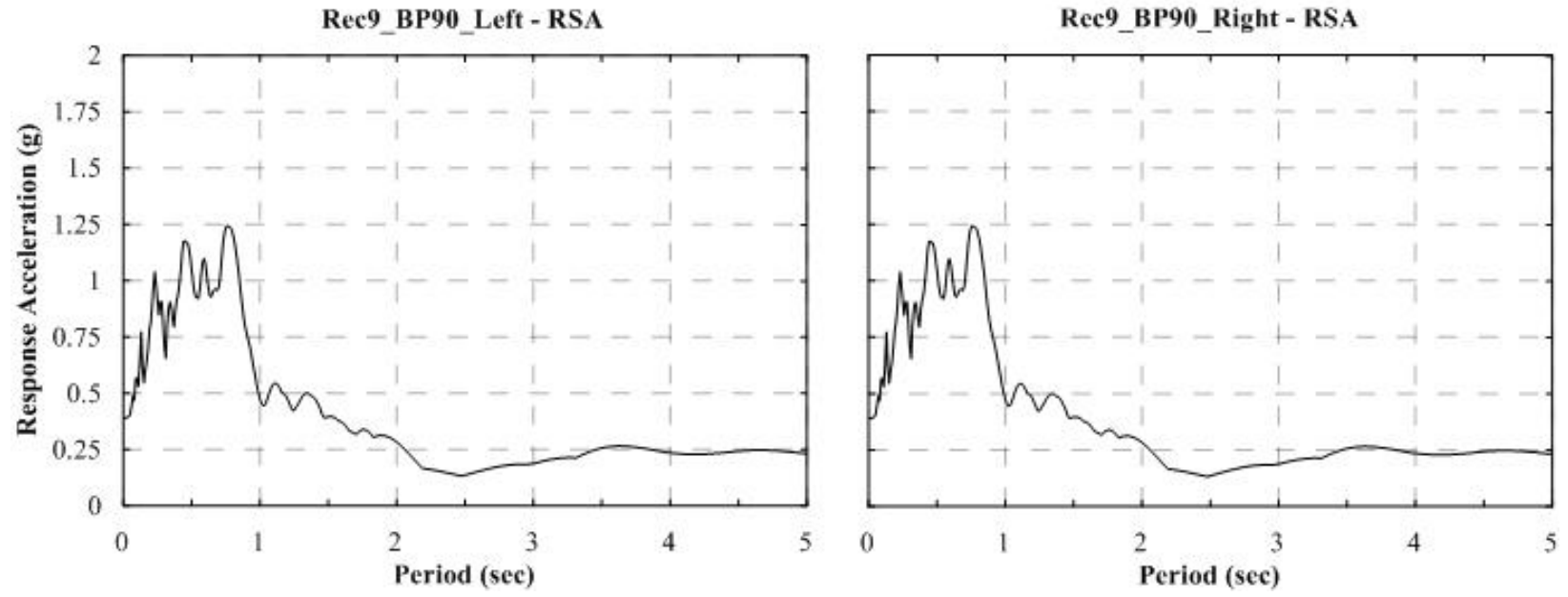

Figure A.36 - Pseudo-Acceleration Response Summary for Record Set 9 in Bridge-Parallel direction 

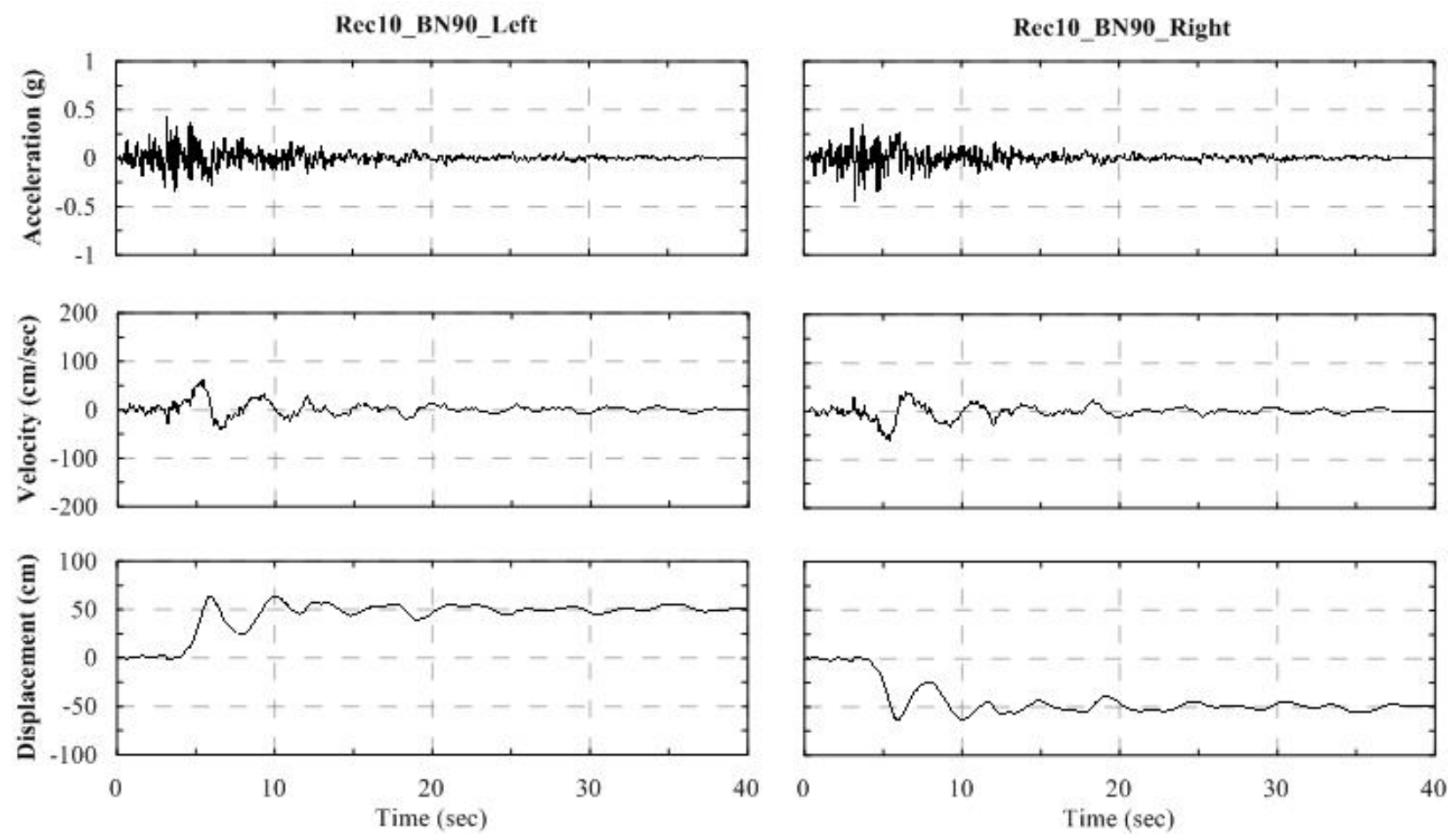

Figure A.37 - Time series summary for Record Set 10 in Bridge-Normal direction
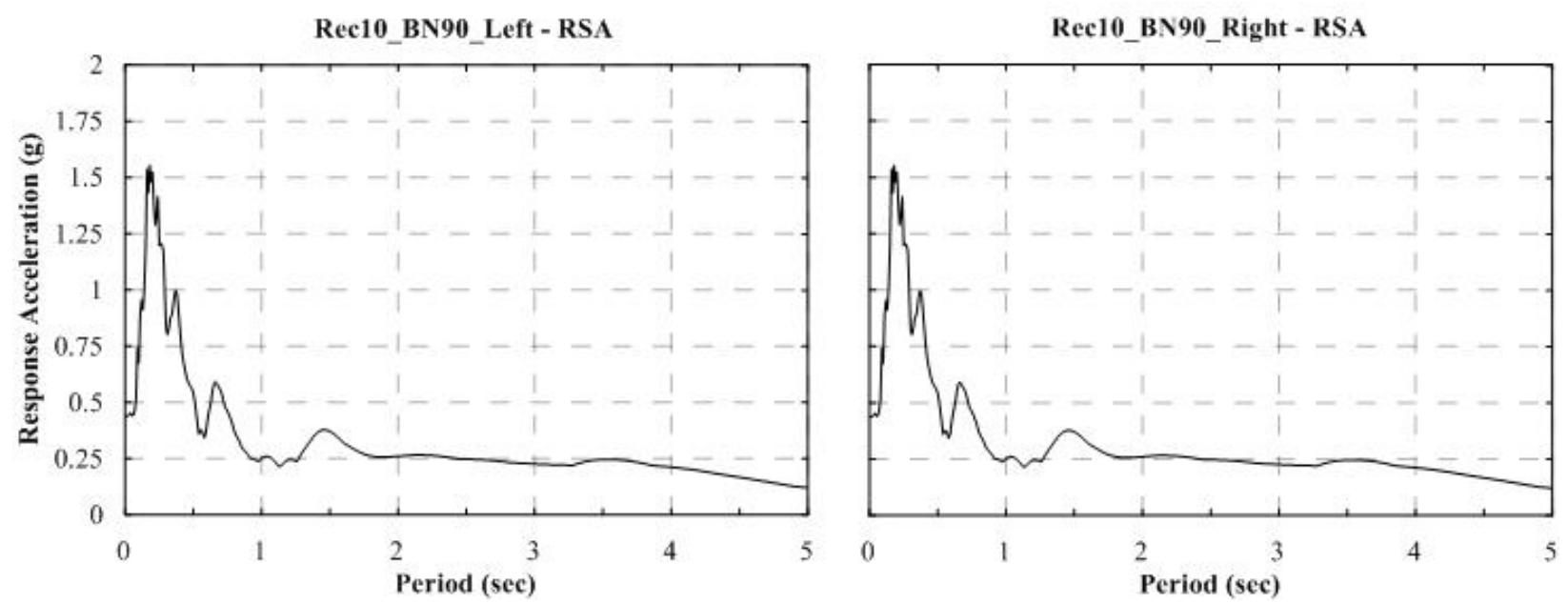

Figure A.38 - Pseudo-Acceleration Response Summary for Record Set 10 in Bridge-Normal direction 

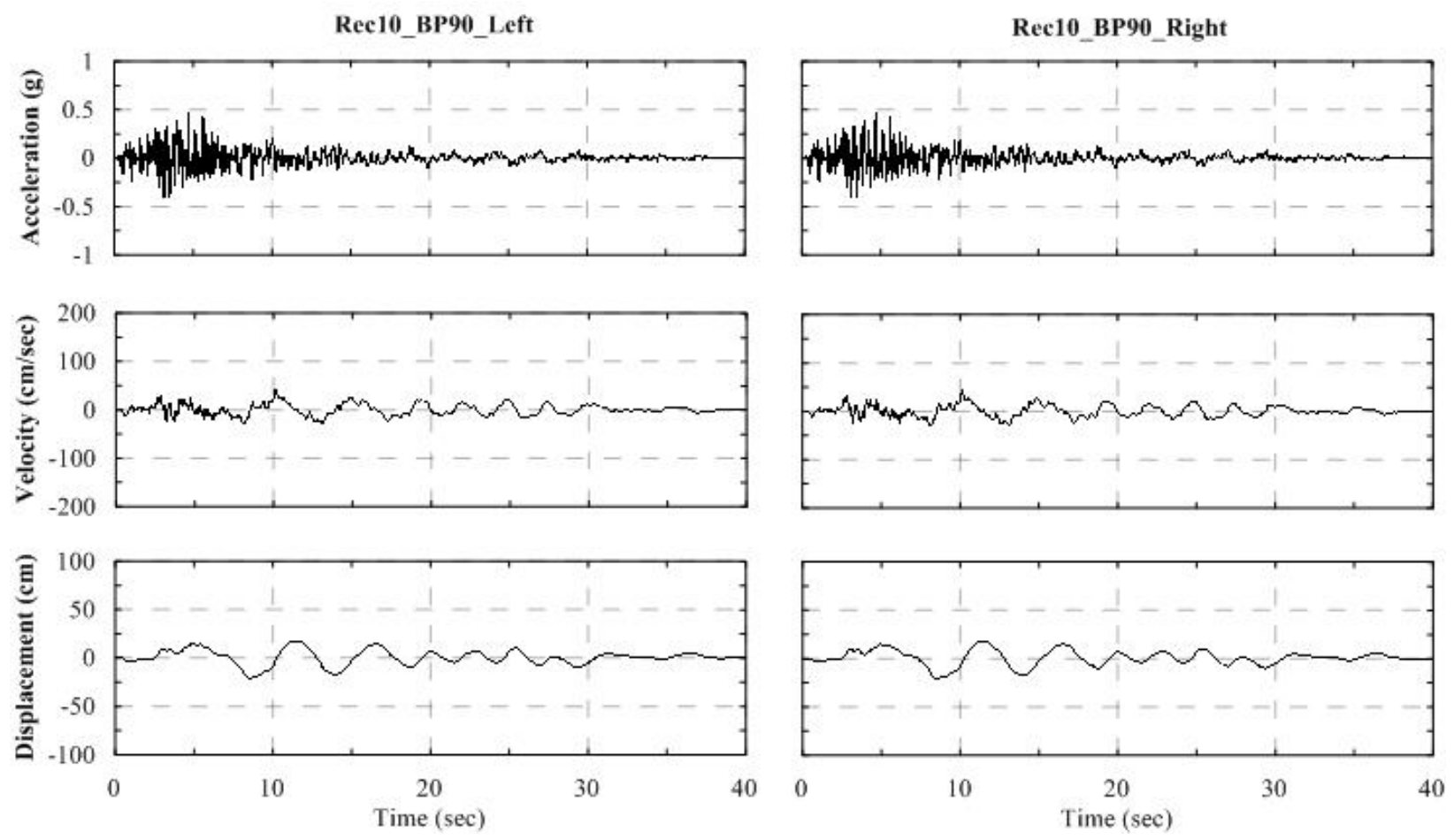

Figure A.39 - Time series summary for Record Set 10 in Bridge-Parallel direction
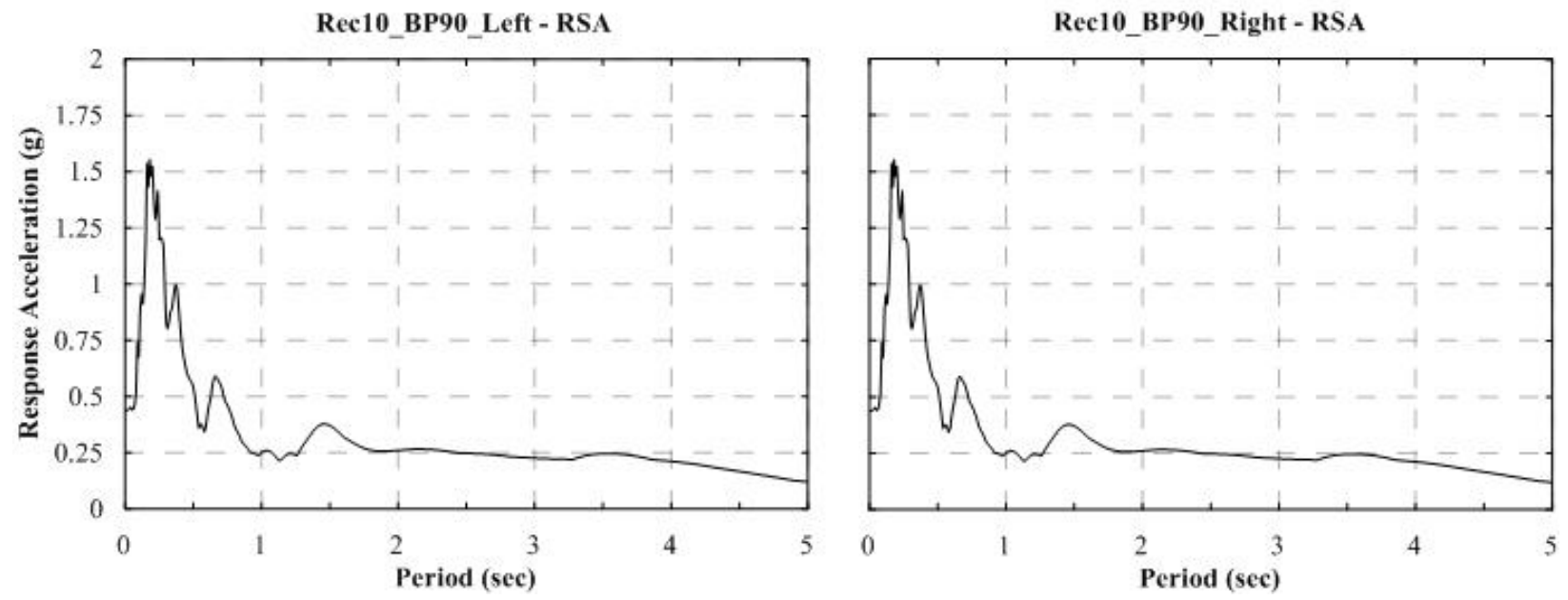

Figure A.40 - Pseudo-Acceleration Response Summary for Record Set 10 in Bridge-Parallel direction 
Appendix B : Mode Shapes and Periods 

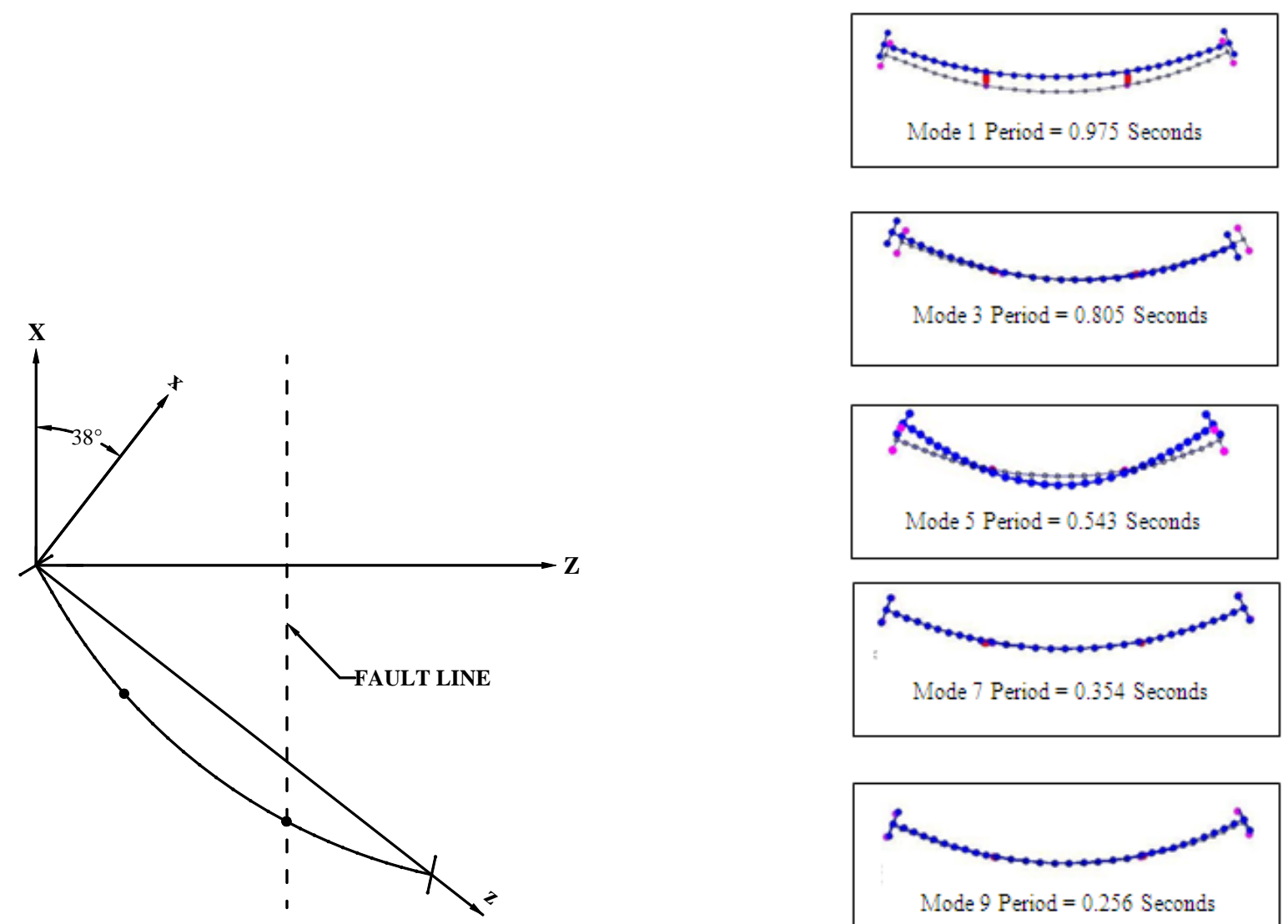

Figure B.1 Sketch of Bridge 55-0837S when $\theta=-38^{\circ}$.
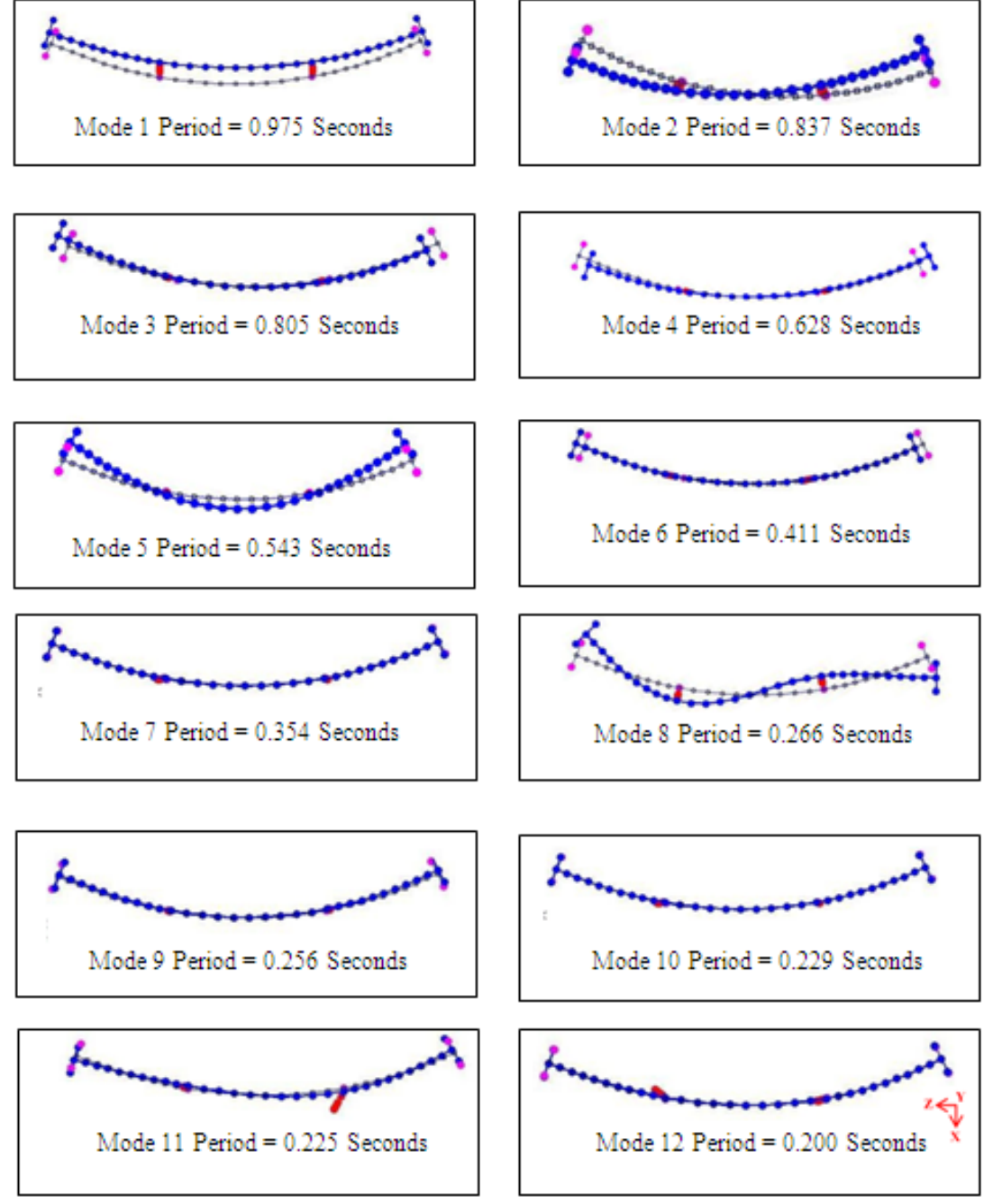

Figure B.2 - Mode Shapes and Period summary for $\theta=-38^{\circ}$ (Longitudinal Abutment Stiffness $=0.10 \mathrm{~K}_{\text {eff }}$ ) 

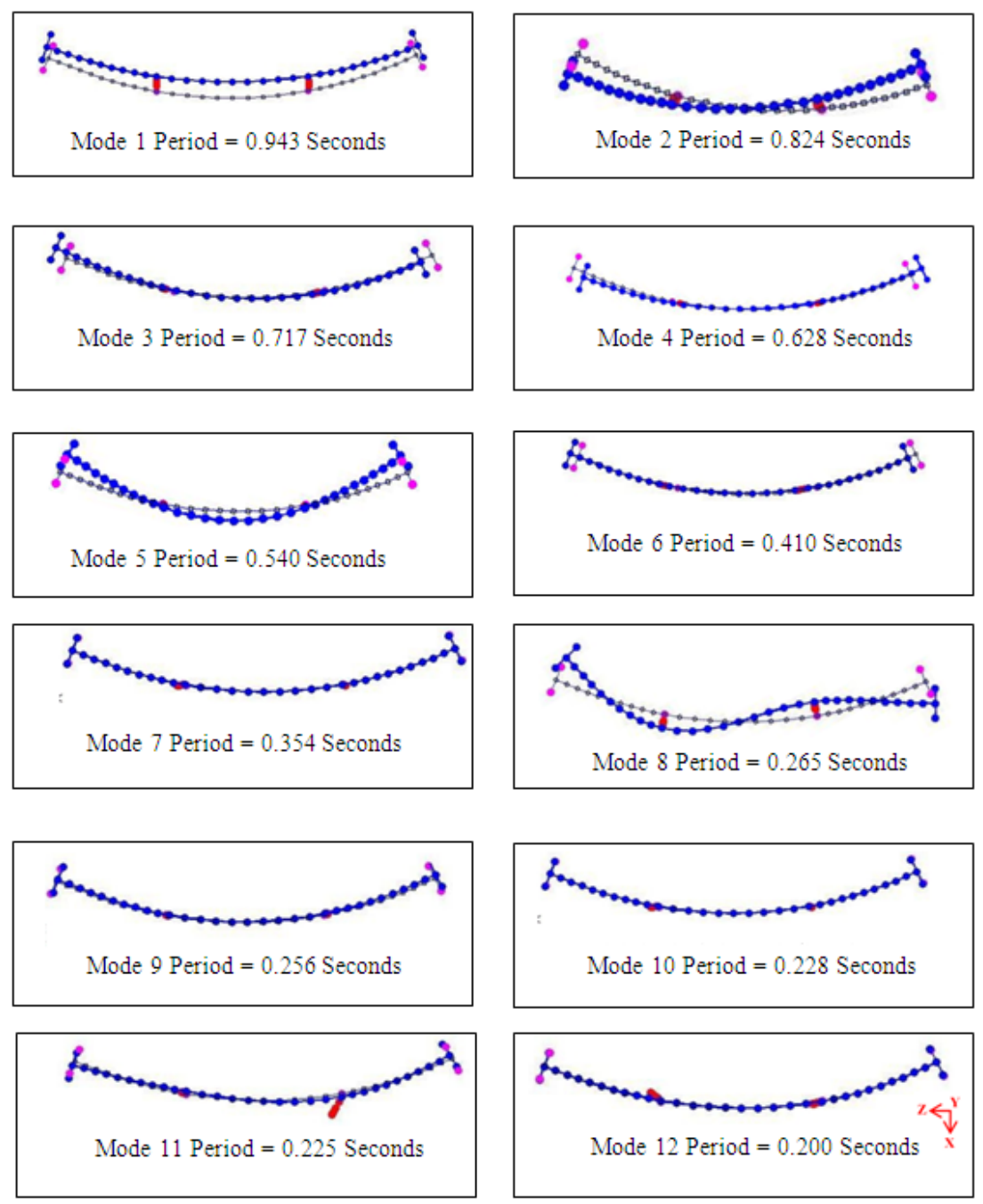

Figure B.3 - Mode Shapes and Period summary for $\theta=-38^{\circ}$ (Longitudinal Abutment Stiffness $=0.55 \mathrm{~K}_{\mathrm{eff}}$ )
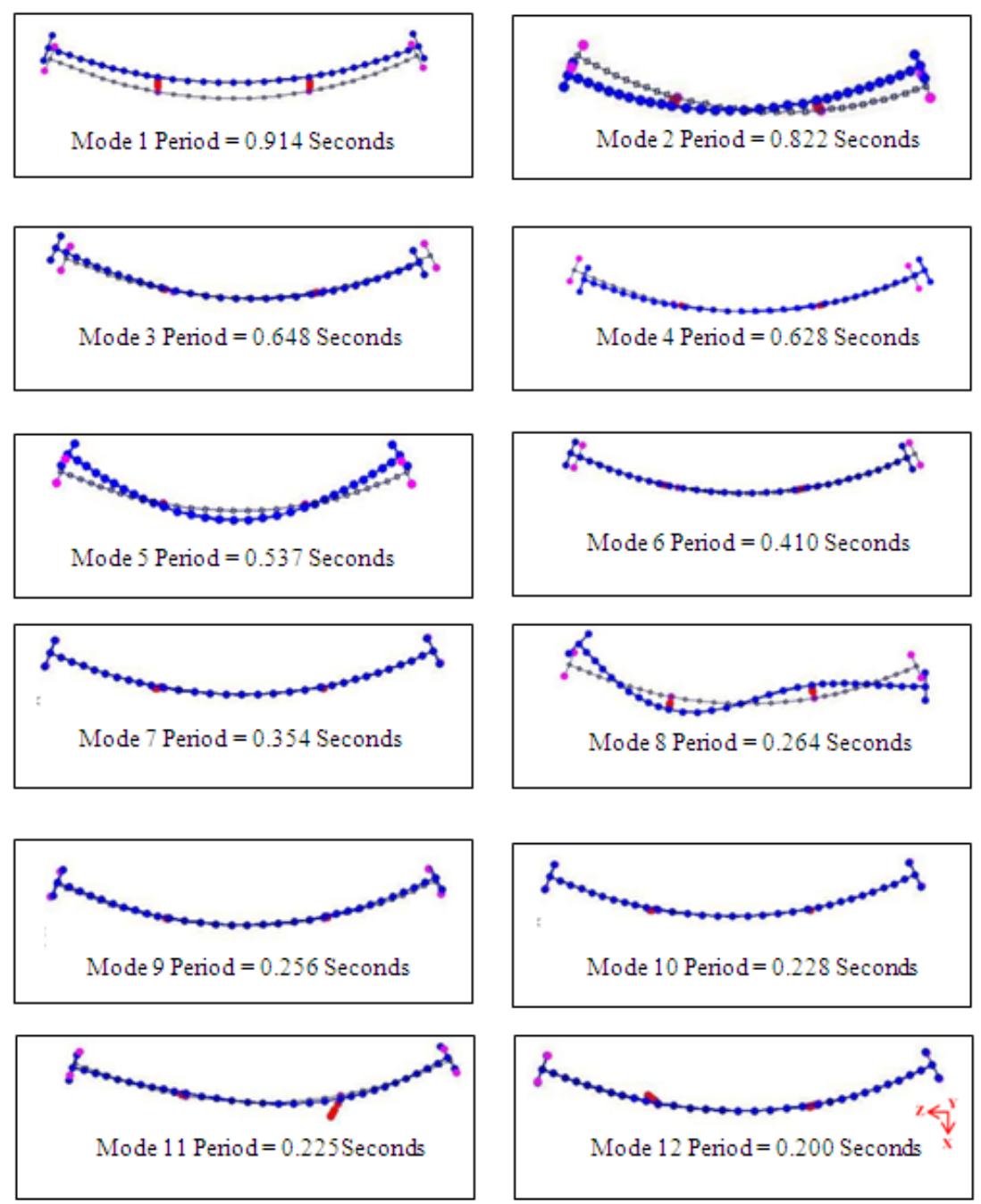

Figure B.4 - Mode Shapes and Period summary for $\theta=-38^{\circ}$ (Longitudinal Abutment Stiffness $=1.00 \mathrm{~K}_{\text {eff }}$ ) 


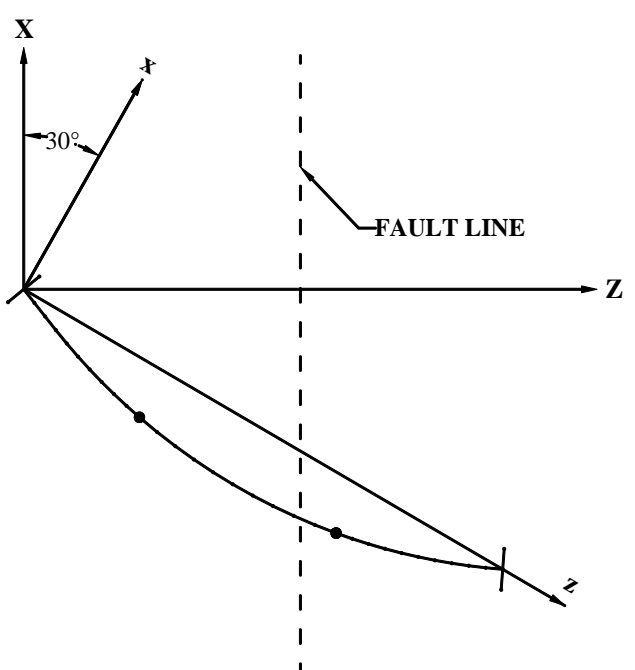

Figure B.5 - Sketch of Bridge 55$0837 \mathrm{~S}$ when $\theta=-30^{\circ}$.
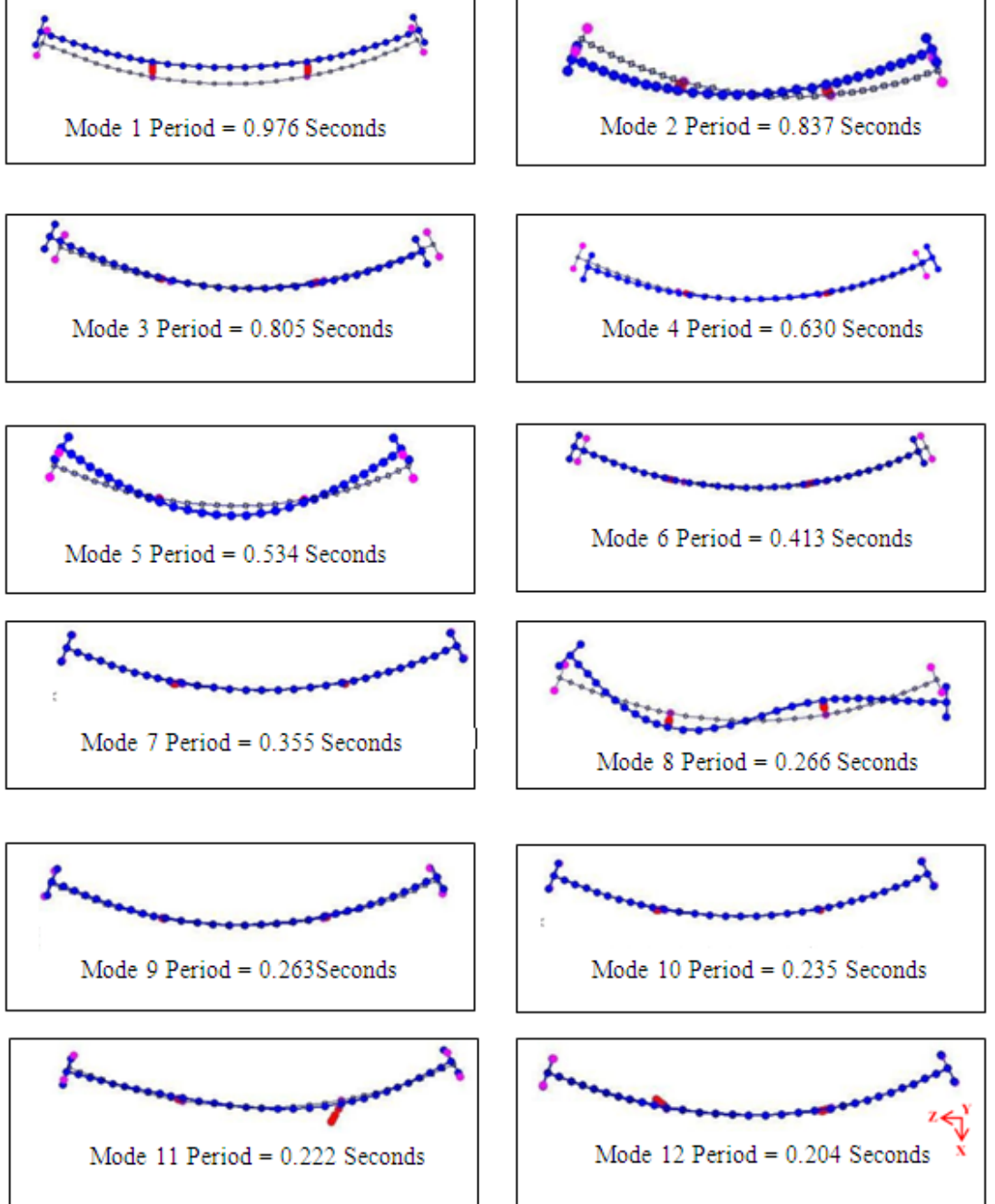

Figure B.6 - Mode Shapes and Period summary for $\theta=-30^{\circ}$ (Longitudinal Abutment Stiffness $=0.10 \mathrm{~K}_{\text {eff }}$ ) 

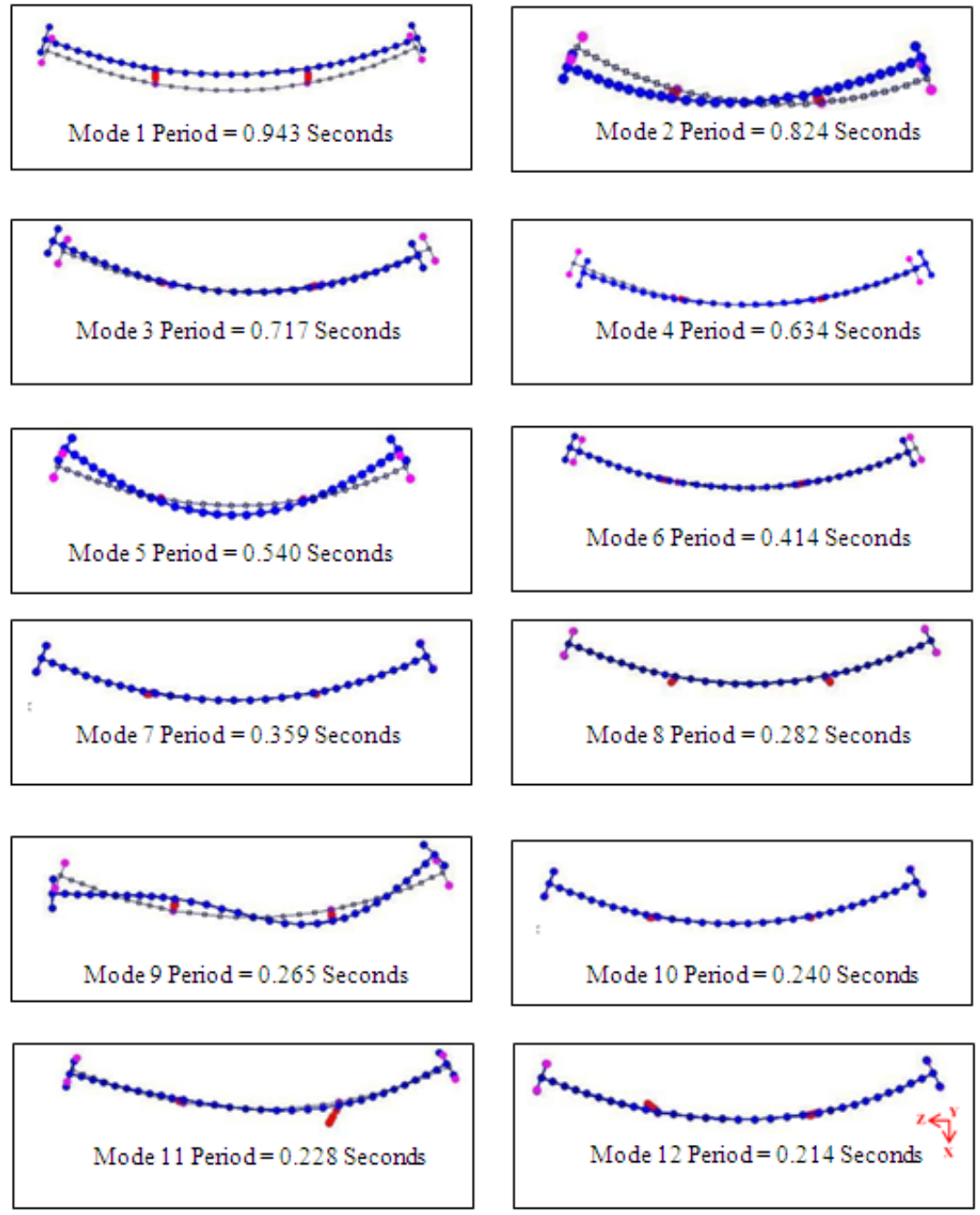

Figure B.7 - Mode Shapes and Period summary for $\theta=-30^{\circ}$ (Longitudinal Abutment Stiffness $=0.55 \mathrm{~K}_{\text {eff }}$ )
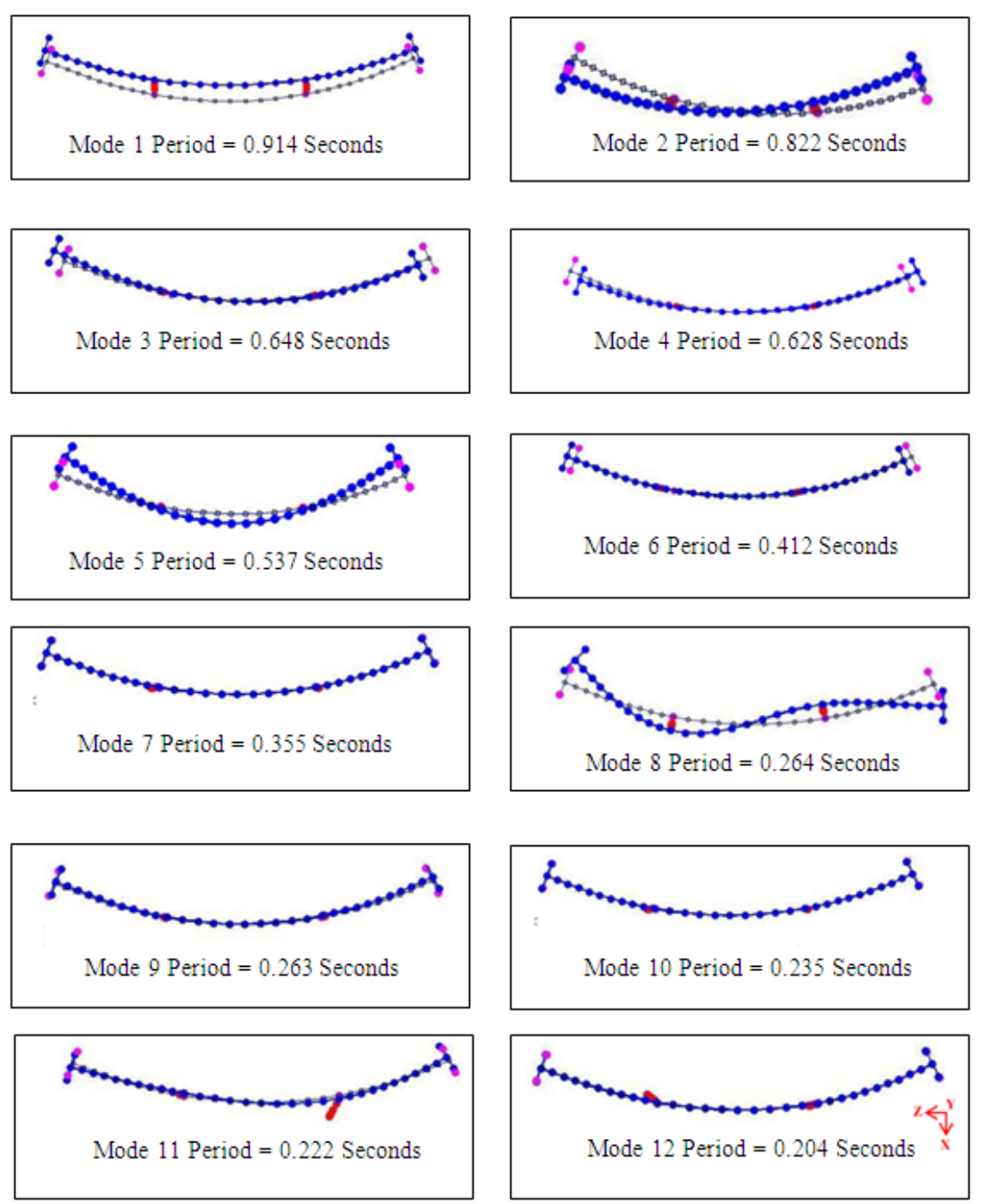

Figure B. 8 - Mode Shapes and Period summary for $\theta=-30^{\circ}$ (Longitudinal Abutment Stiffness= $1.00 \mathrm{~K}_{\text {eff }}$ ) 


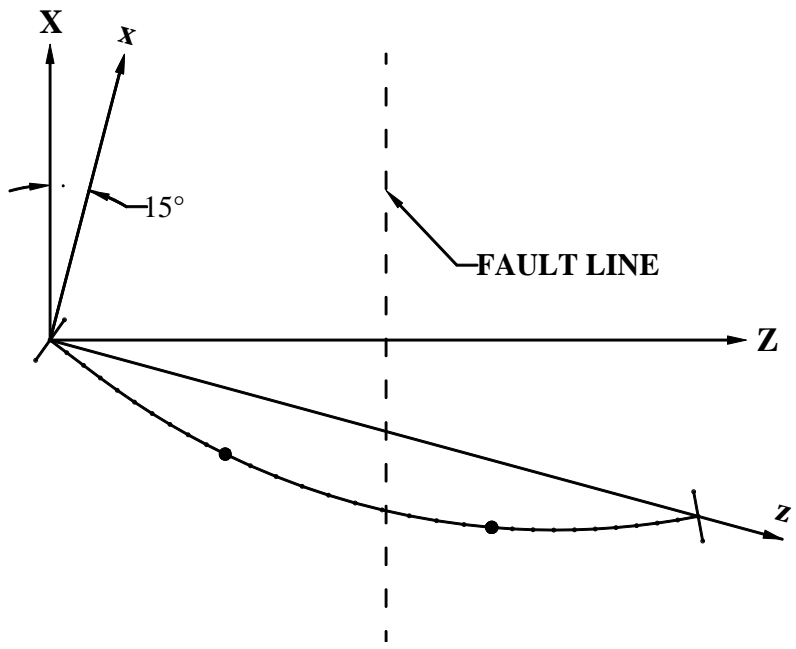

Figure B.9 - Sketch of Bridge 55-0837S when $\theta=-15^{\circ}$.
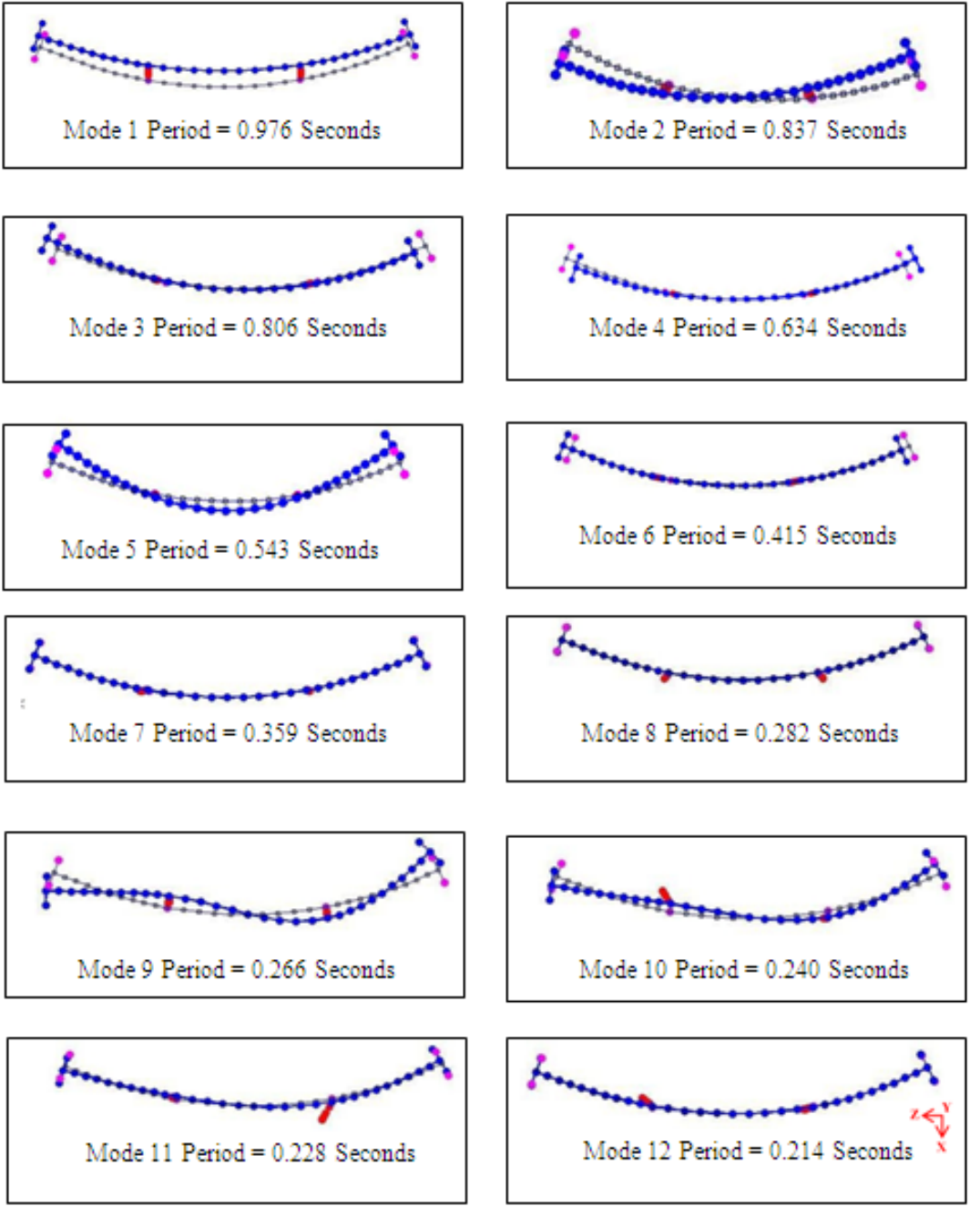

Figure B.10 - Mode Shapes and Period summary for $\theta=-15^{\circ}$ (Longitudinal Abutment Stiffness $=0.10 \mathrm{~K}_{\text {eff }}$ ) 

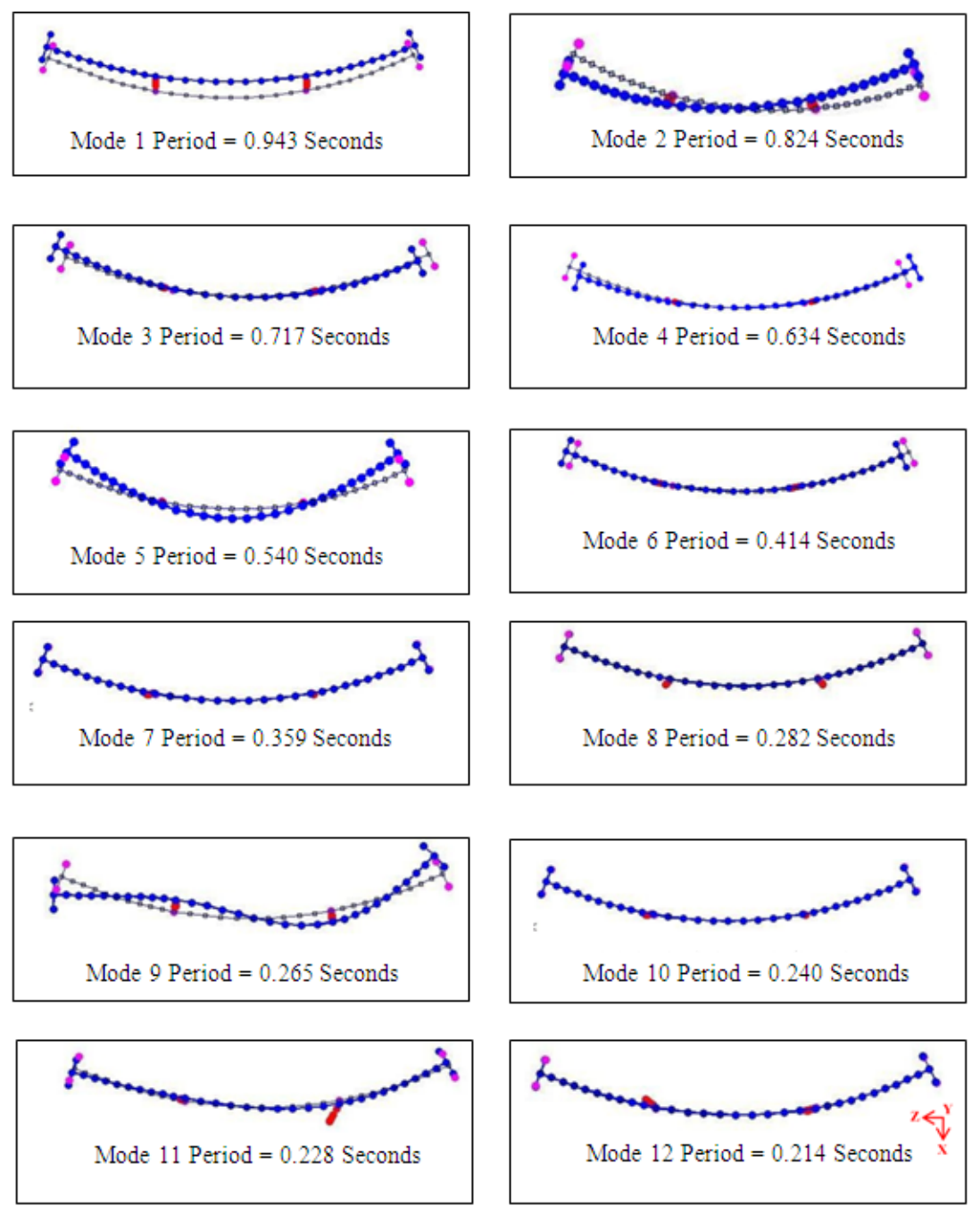

Figure B.11 - Mode Shapes and Period summary for $\theta=-15^{\circ}$ (Longitudinal Abutment Stiffness $=0.55 \mathrm{~K}_{\mathrm{eff}}$ )
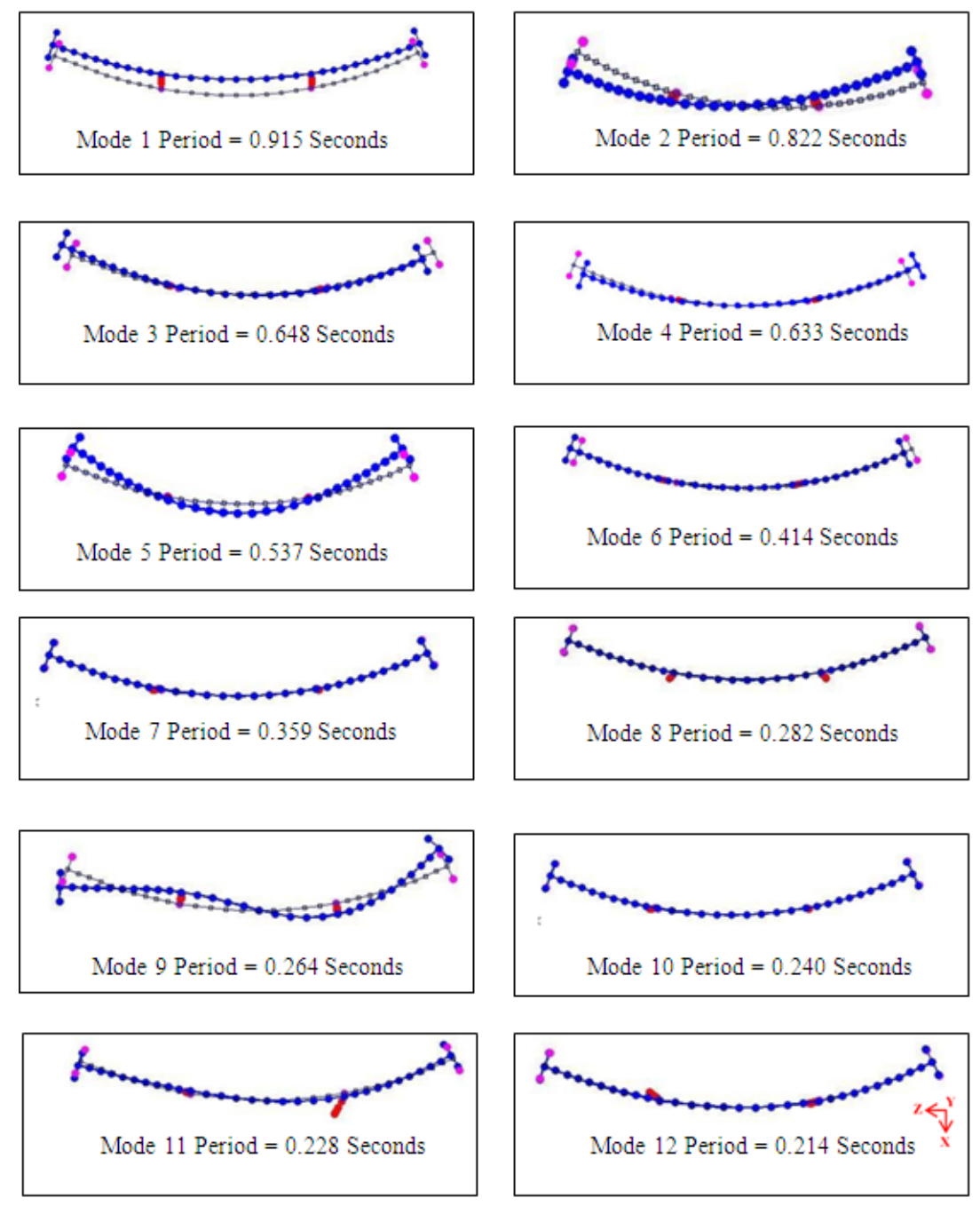

Figure B.12 - Mode Shapes and Period summary for $\theta=-15^{\circ}$ (Longitudinal Abutment Stiffness $=1.00 \mathrm{~K}_{\mathrm{eff}}$ ) 

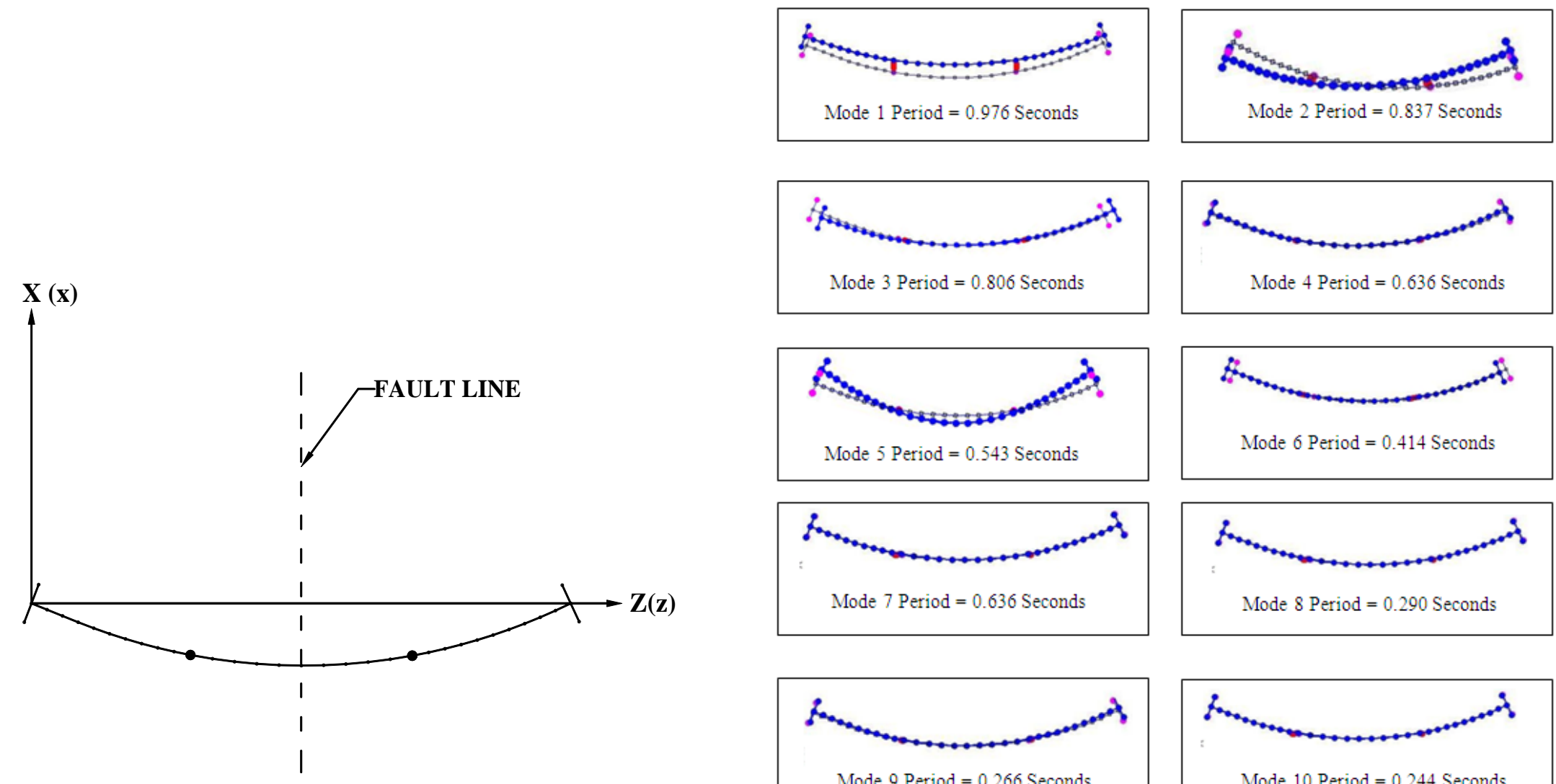

Figure B.13- Sketch of Bridge 55-0837S when $\theta=0^{\circ}$
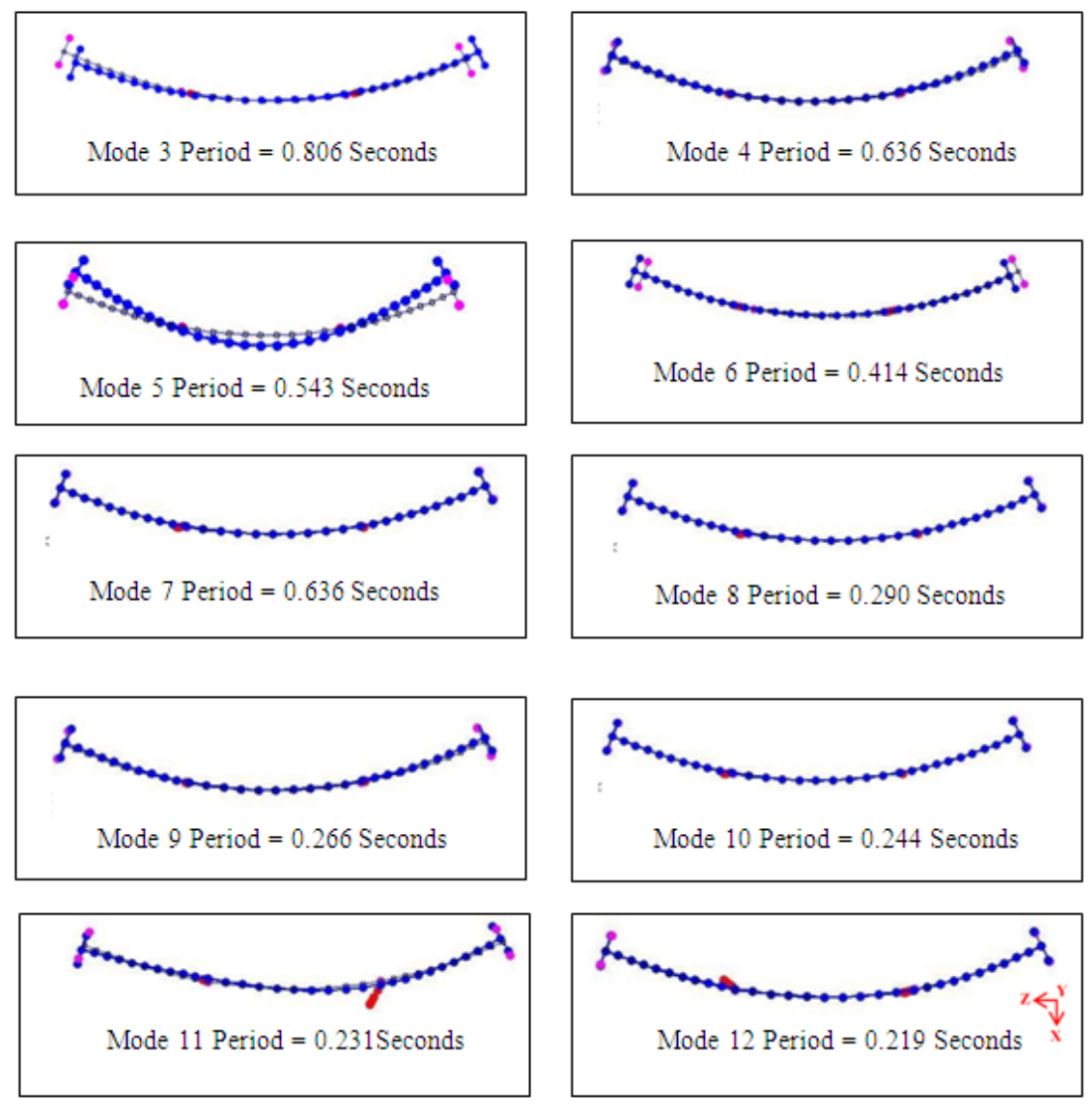

Figure B.14 - Mode Shapes and Period summary for $\theta=0^{\circ}$ (Longitudinal Abutment Stiffness $=0.10 \mathrm{~K}_{\mathrm{eff}}$ ) 

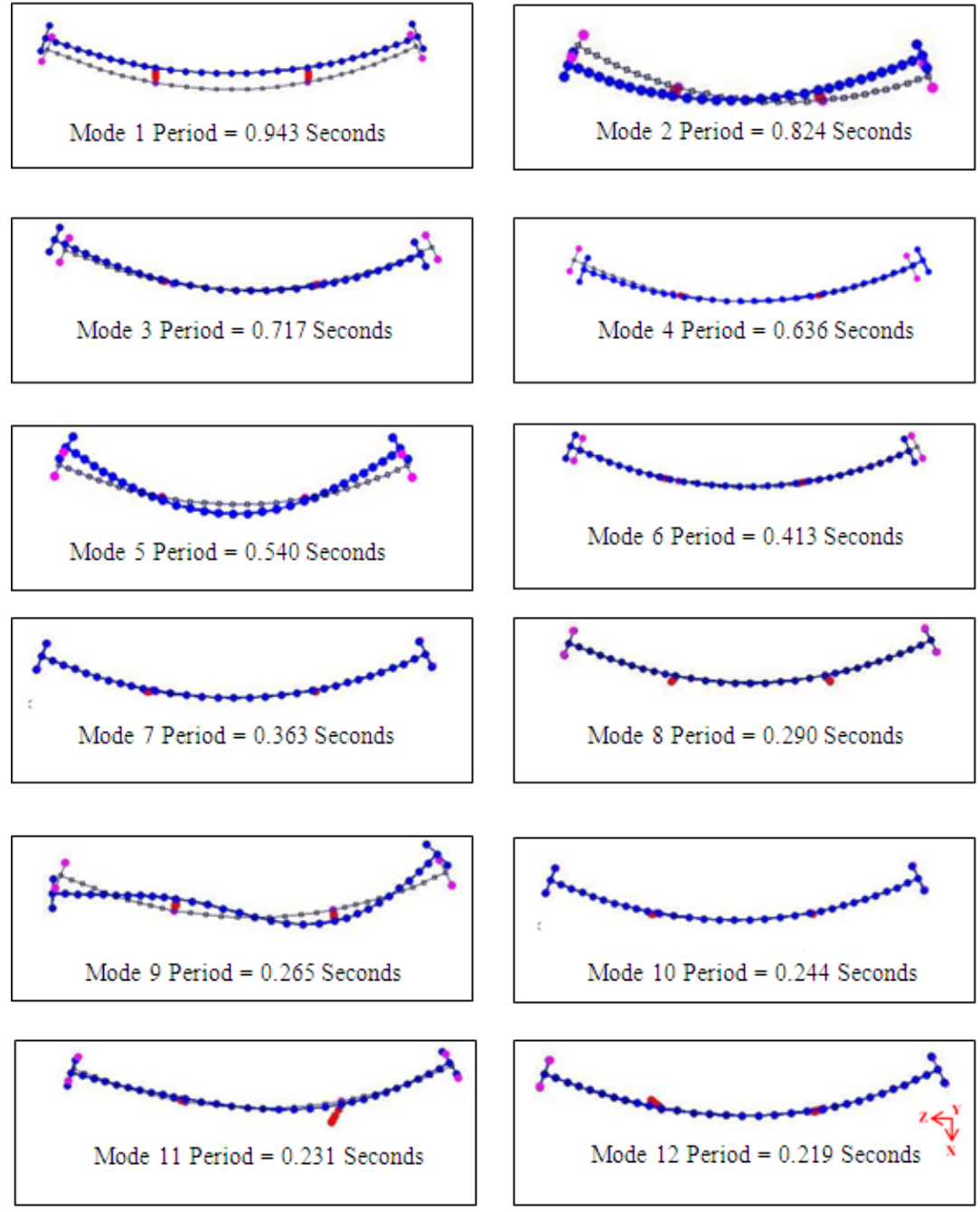

Figure B.15 - Mode Shapes and Period summary for $\theta=0^{\circ}$ (Longitudinal Abutment Stiffness $=0.55 \mathrm{~K}_{\mathrm{eff}}$ )
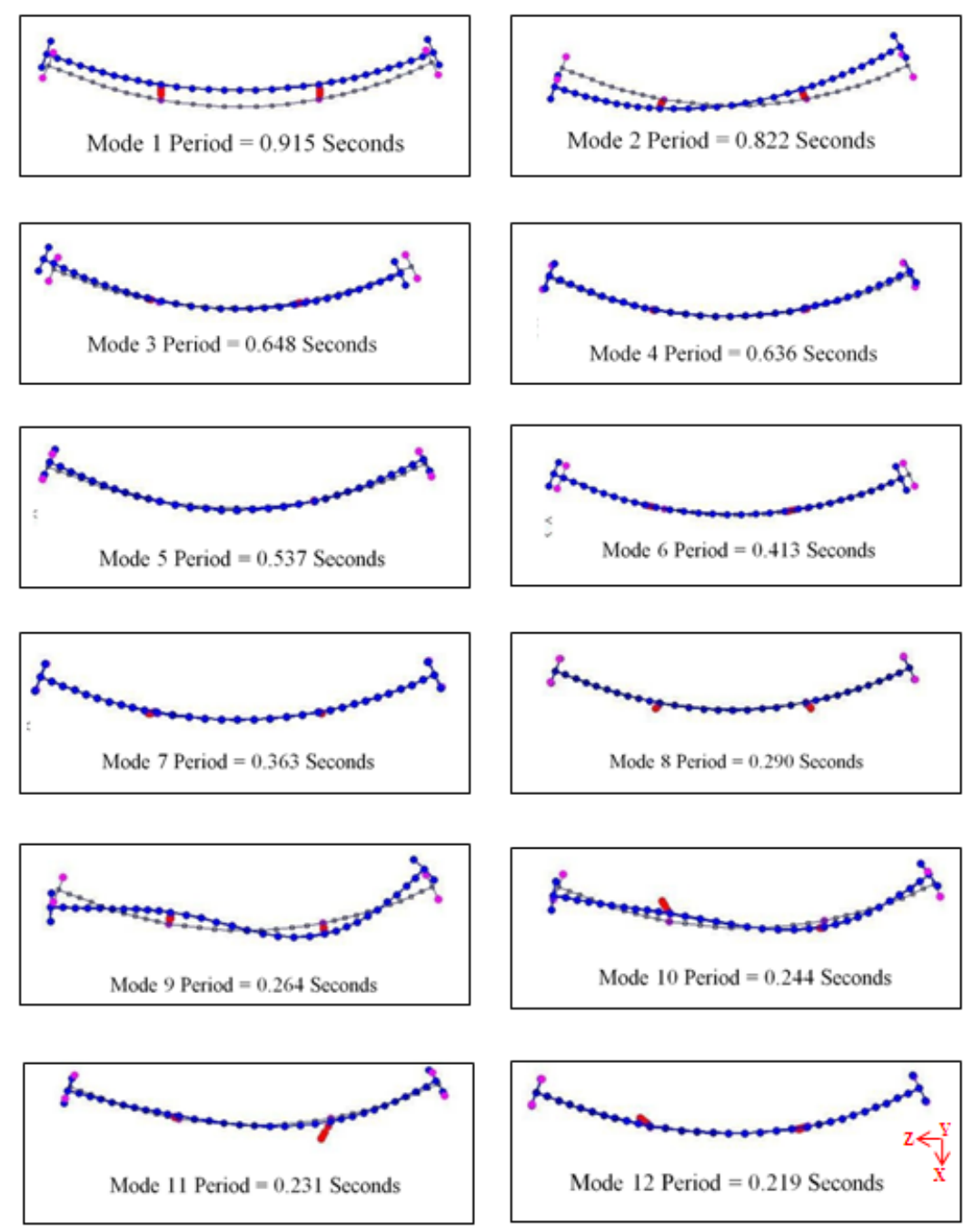

Figure B.16 - Mode Shapes and Period summary for $\theta=0^{\circ}$ (Longitudinal Abutment Stiffness $=1.00 \mathrm{~K}_{\text {eff }}$ ) 


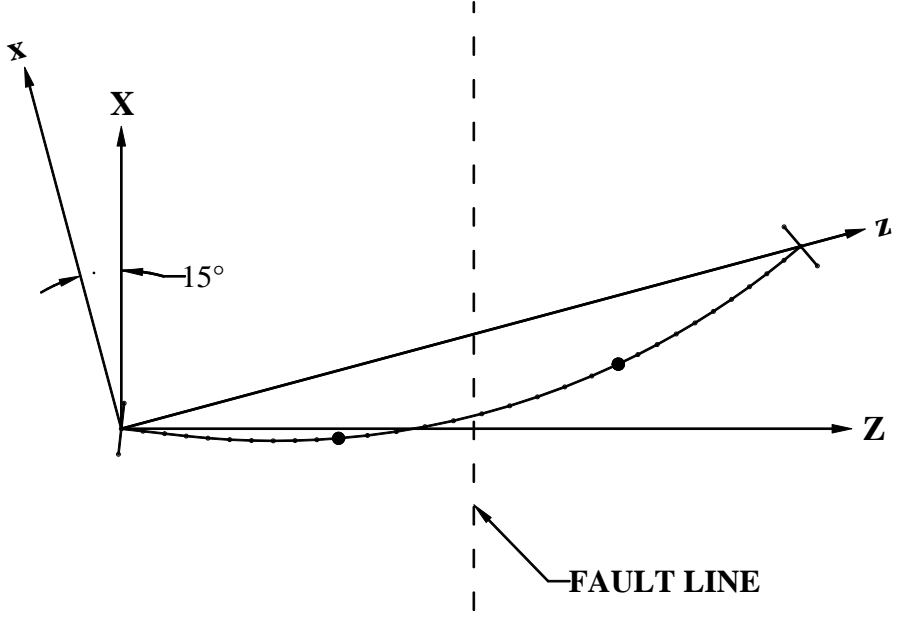

Figure B.17 - Sketch of Bridge 55-0837S when $\theta=15^{\circ}$
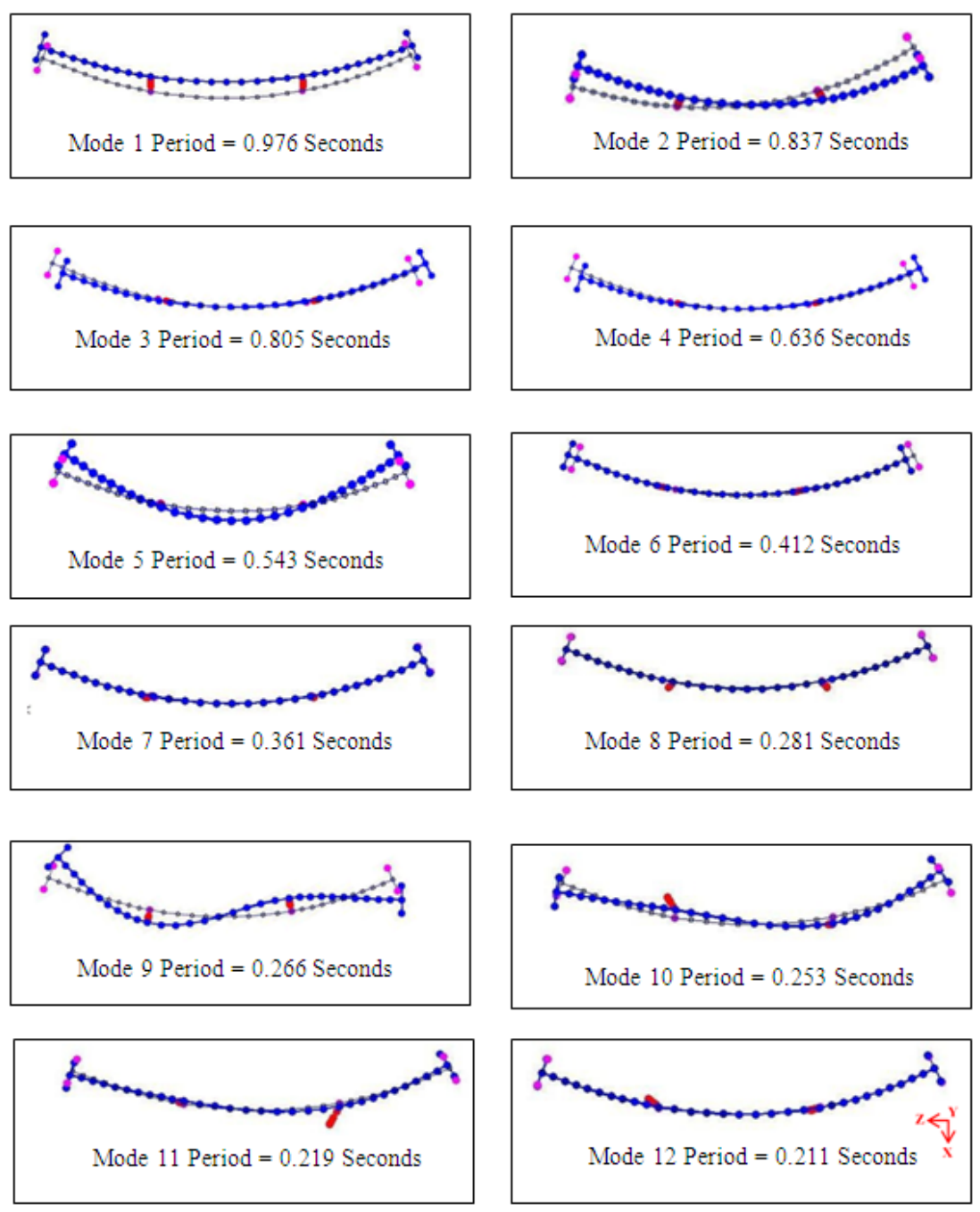

Figure B.18 - Mode Shapes and Period summary for $\theta=15^{\circ}$ (Longitudinal Abutment Stiffness=0.10K $\mathrm{K}_{\mathrm{eff}}$ ) 

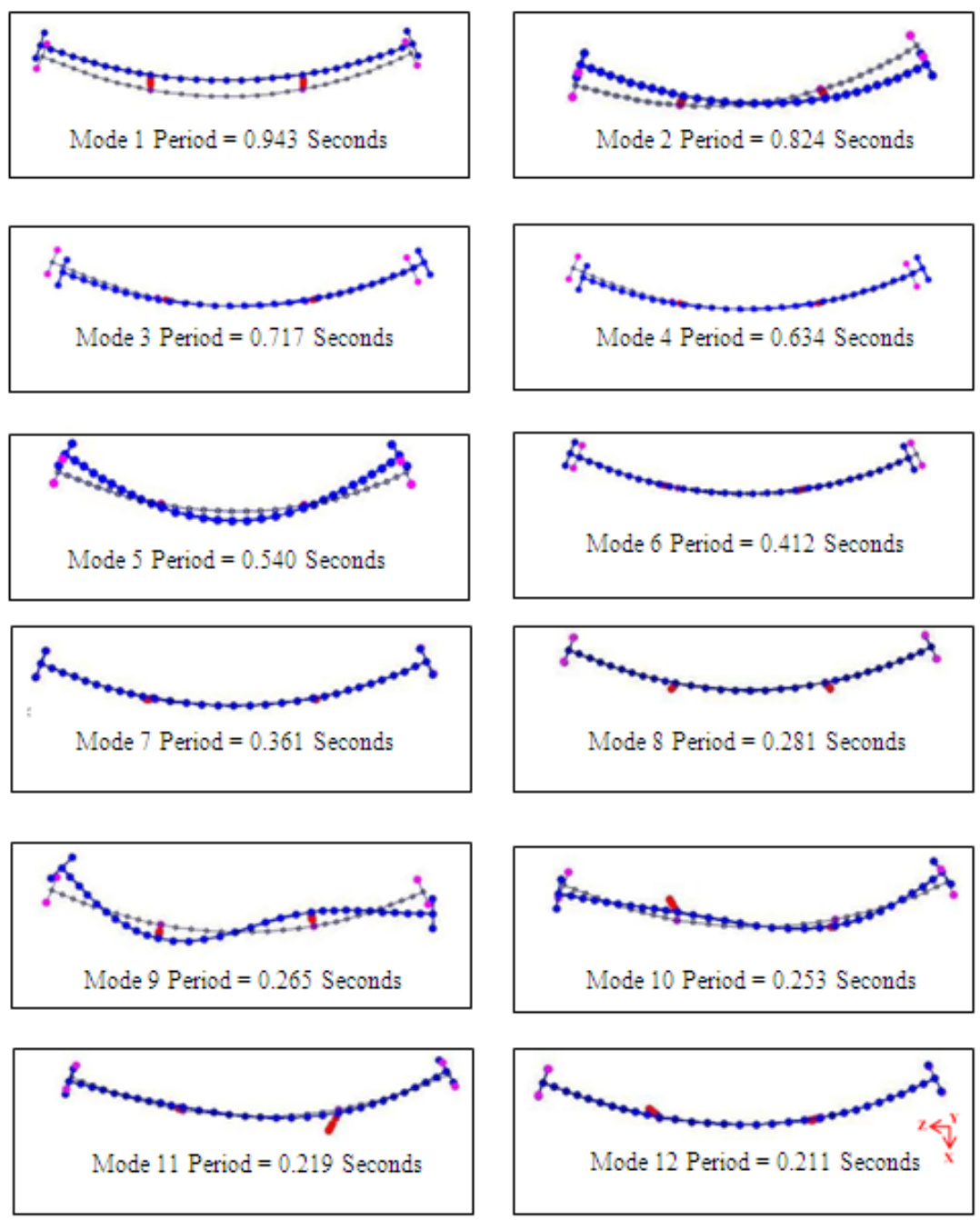

Figure B.19 - Mode Shapes and Period summary for $\theta=15^{\circ}$ (Longitudinal Abutment Stiffness $=0.55 \mathrm{~K}_{\text {eff }}$ )
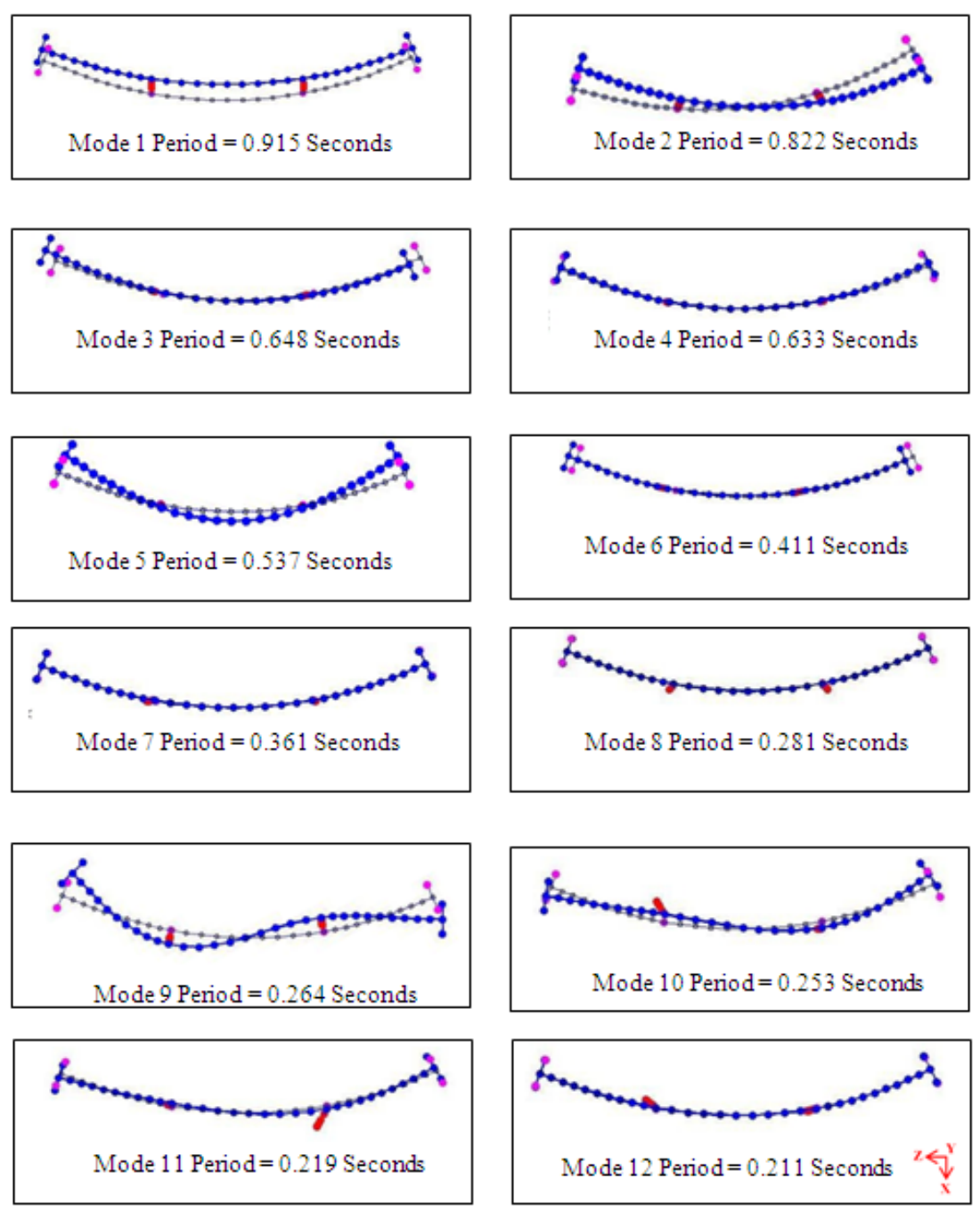

Figure B.20 - Mode Shapes and Period summary for $\theta=15^{\circ}$ (Longitudinal Abutment Stiffness= $1.00 \mathrm{~K}_{\text {eff }}$ ) 

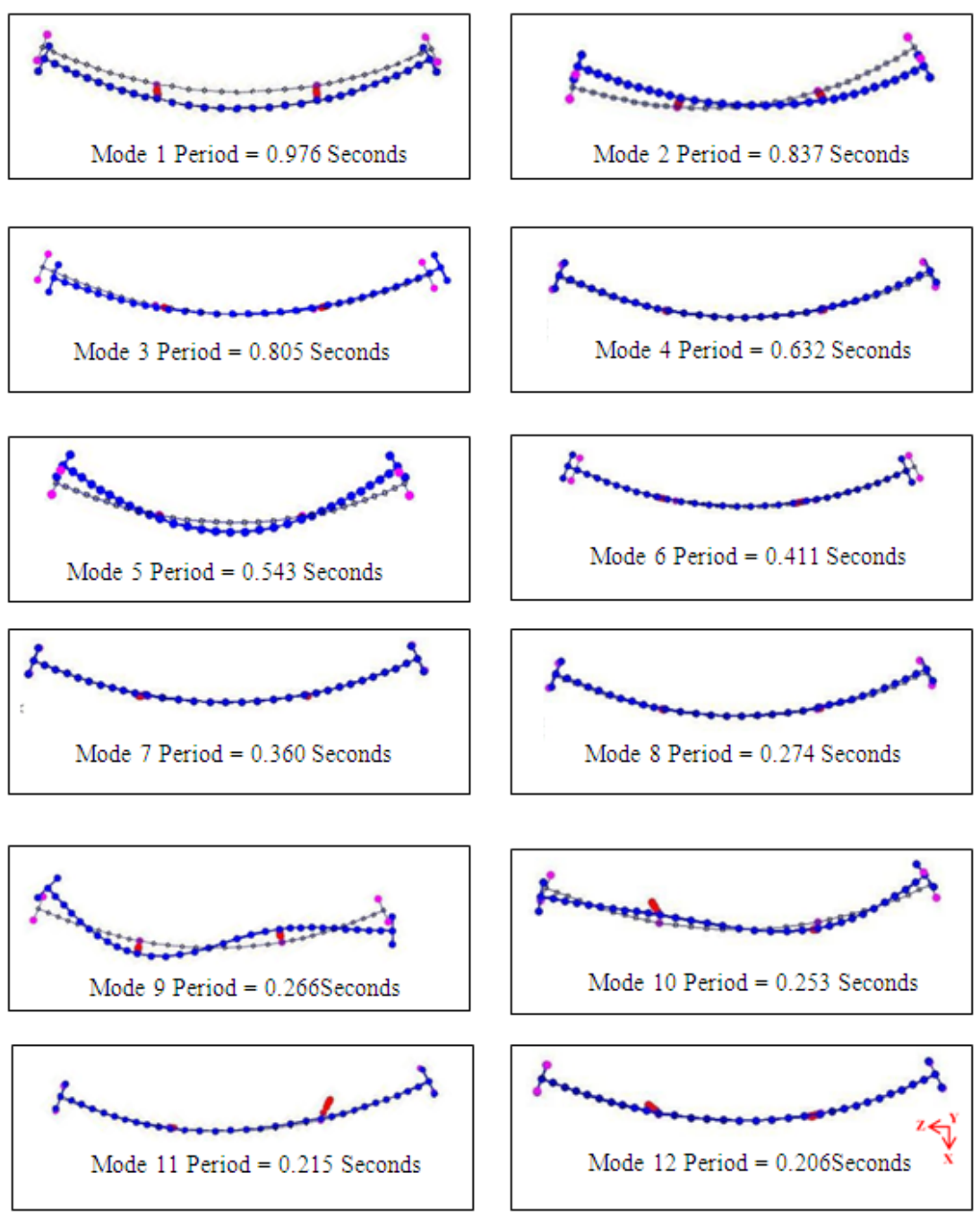

Figure B.22 - Mode Shapes and Period summary for $\theta=21^{\circ}$ (Longitudinal Abutment Stiffness $=0.10 \mathrm{~K}_{\text {eff }}$ ) 

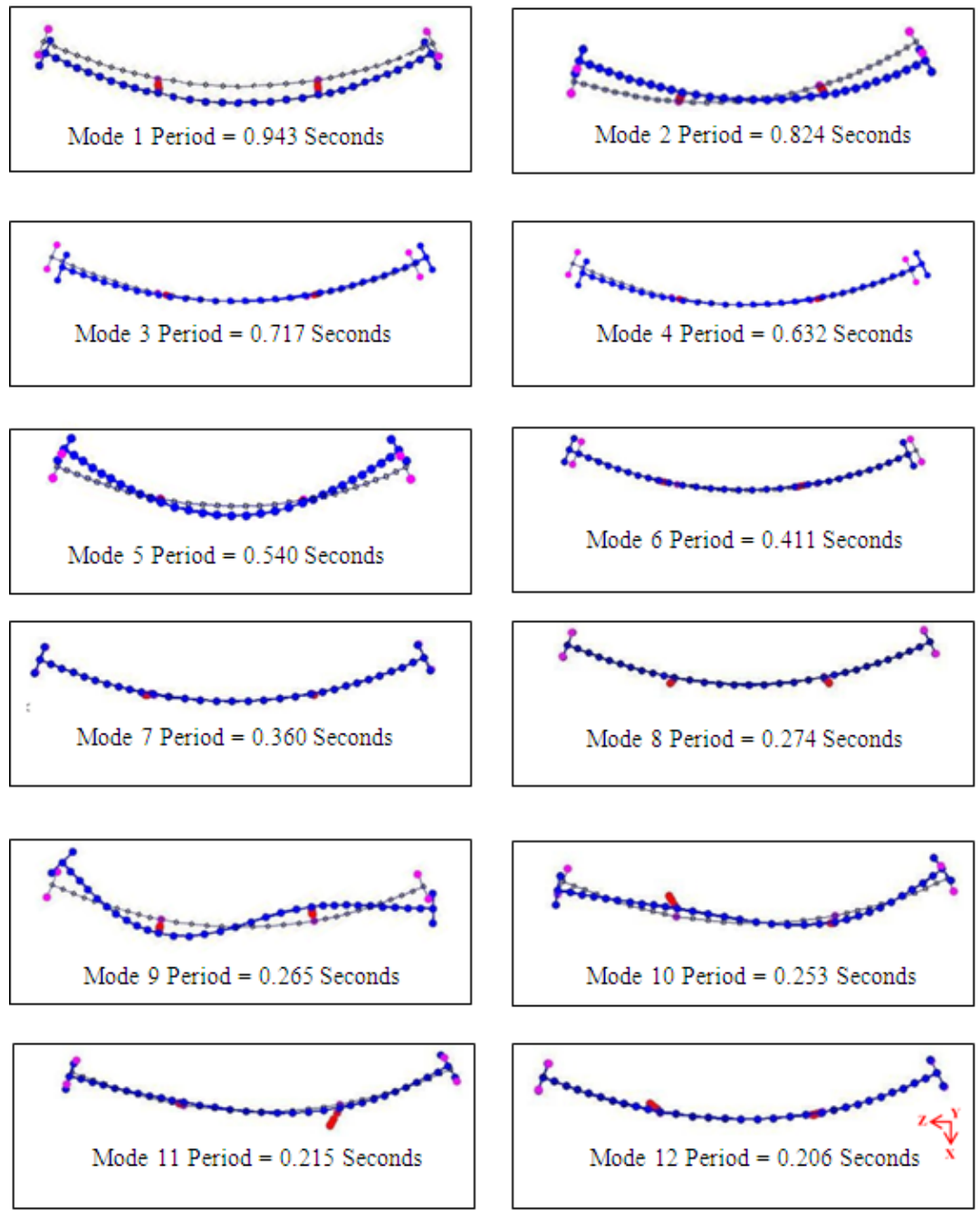

Figure B.23 - Mode Shapes and Period summary for $\theta=21^{\circ}$ (Longitudinal Abutment Stiffness $=0.55 \mathrm{~K}_{\mathrm{eff}}$ )
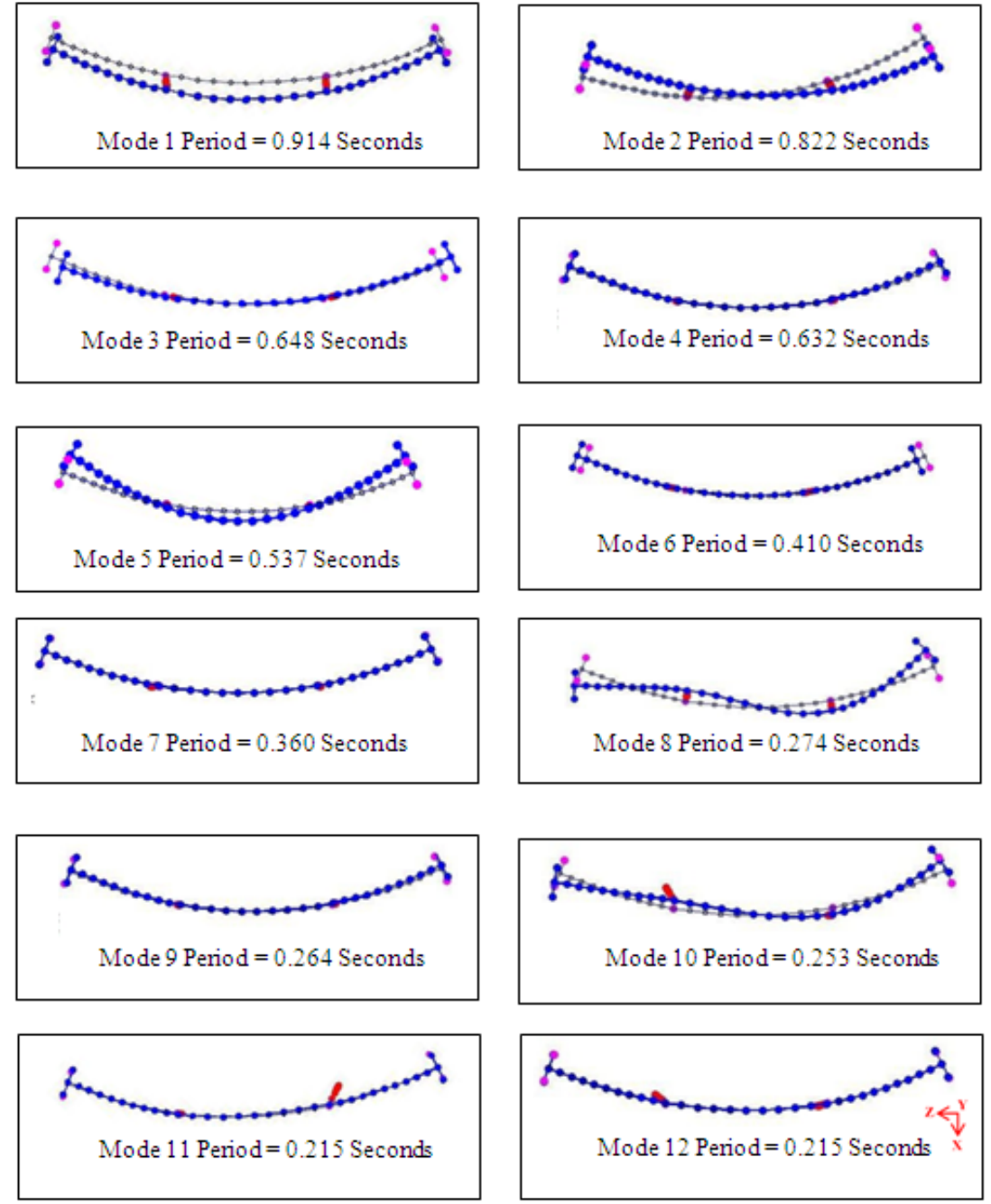

Figure B.24 - Mode Shapes and Period summary for $\theta=21^{\circ}$ (Longitudinal Abutment Stiffness $=1.00 \mathrm{~K}_{\mathrm{eff}}$ ) 

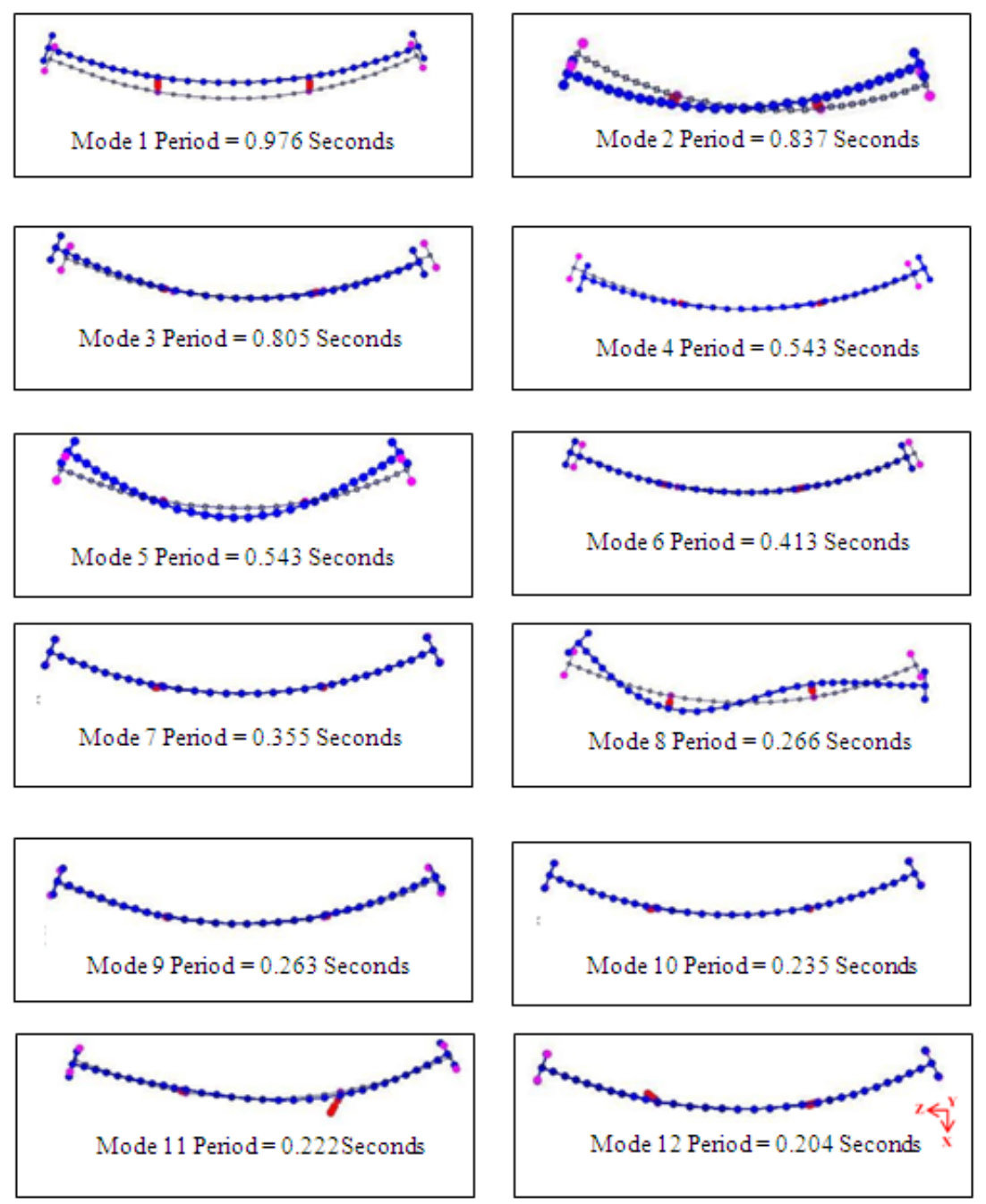

Figure B.26 - Mode Shapes and Period summary for $\theta=30^{\circ}$ (Longitudinal Abutment Stiffness $=0.10 \mathrm{~K}_{\text {eff }}$ ) 

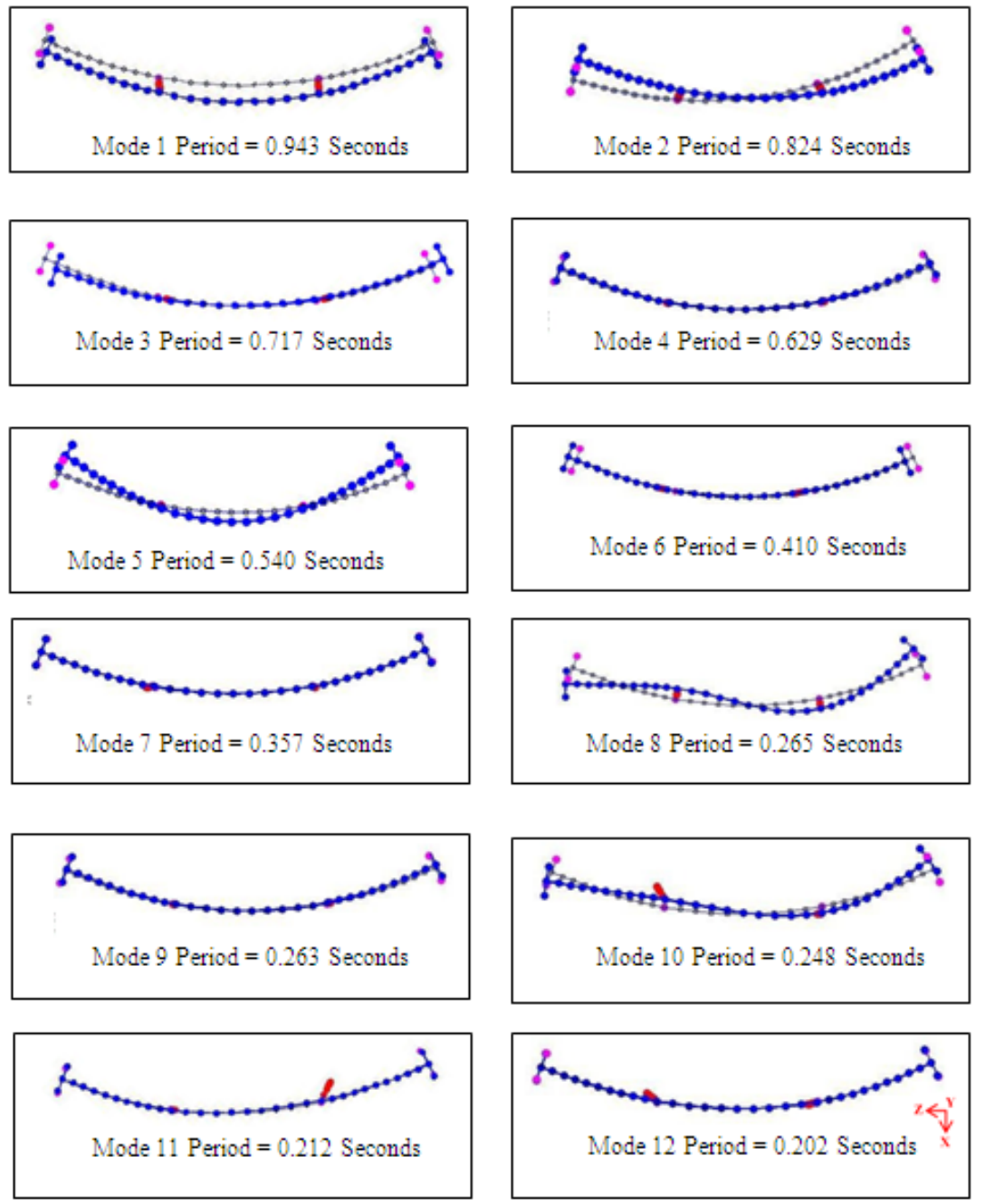

Figure B.27 Mode Shapes and Period summary for $\theta=30^{\circ}$ (Longitudinal Abutment Stiffness $=0.55 \mathrm{~K}_{\mathrm{eff}}$ )
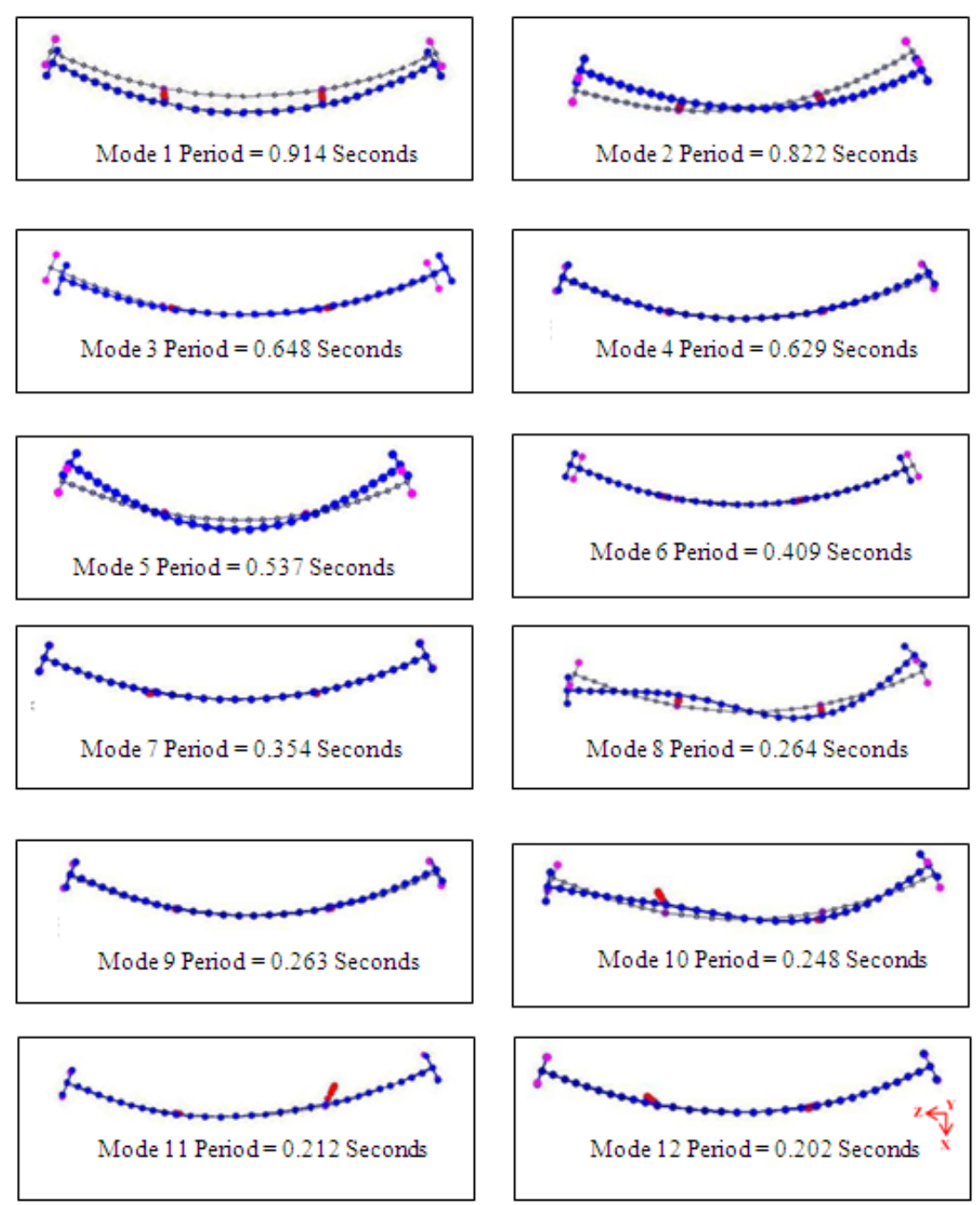

Figure B.28 - Mode Shapes and Period summary for $\theta=30^{\circ}$ (Longitudinal Abutment Stiffness= $1.00 \mathrm{~K}_{\text {eff }}$ ) 

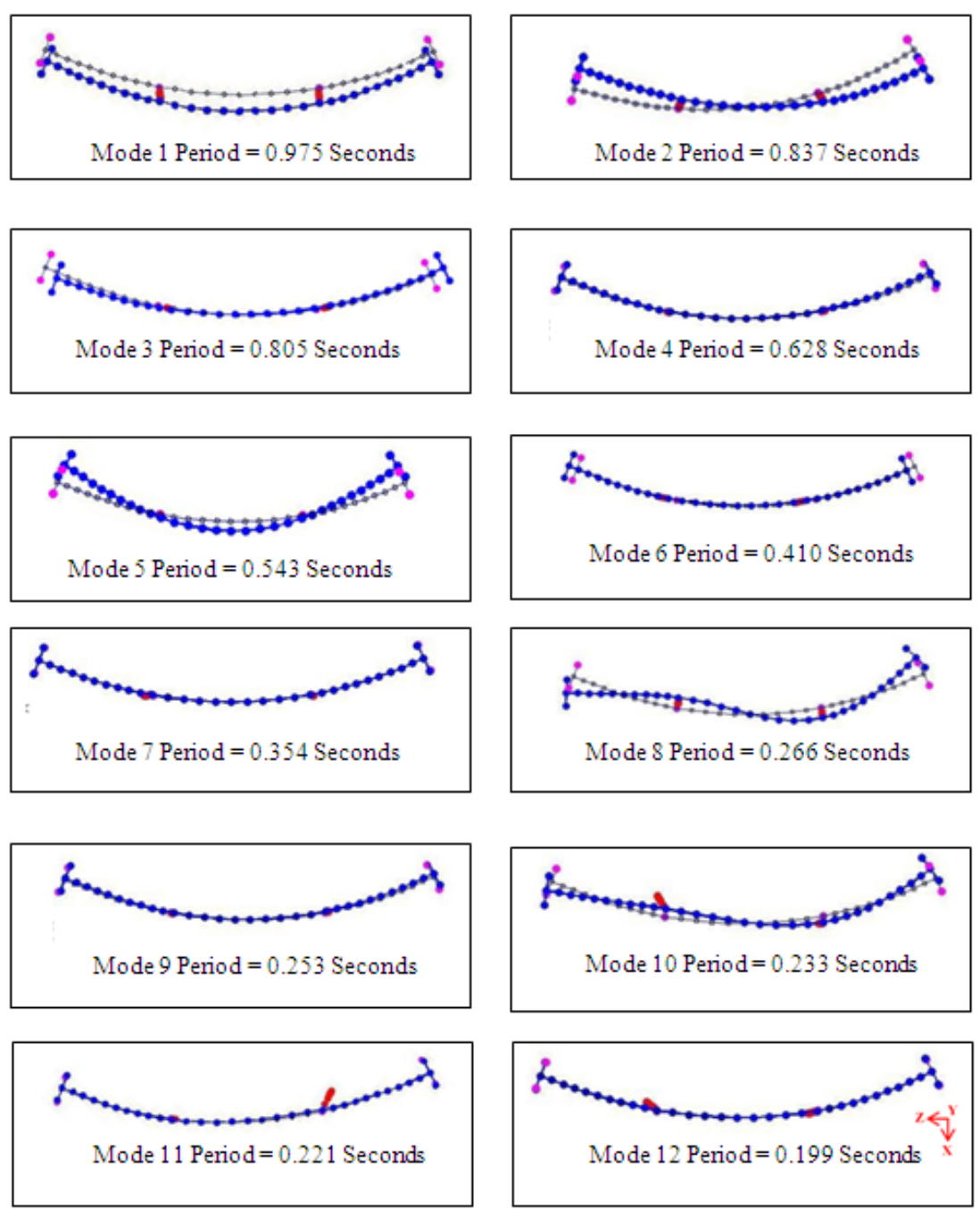

Figure B.30 - Mode Shapes and Period summary for $\theta=45^{\circ}$ (Longitudinal Abutment Stiffness $=0.10 \mathrm{~K}_{\text {eff }}$ ) 

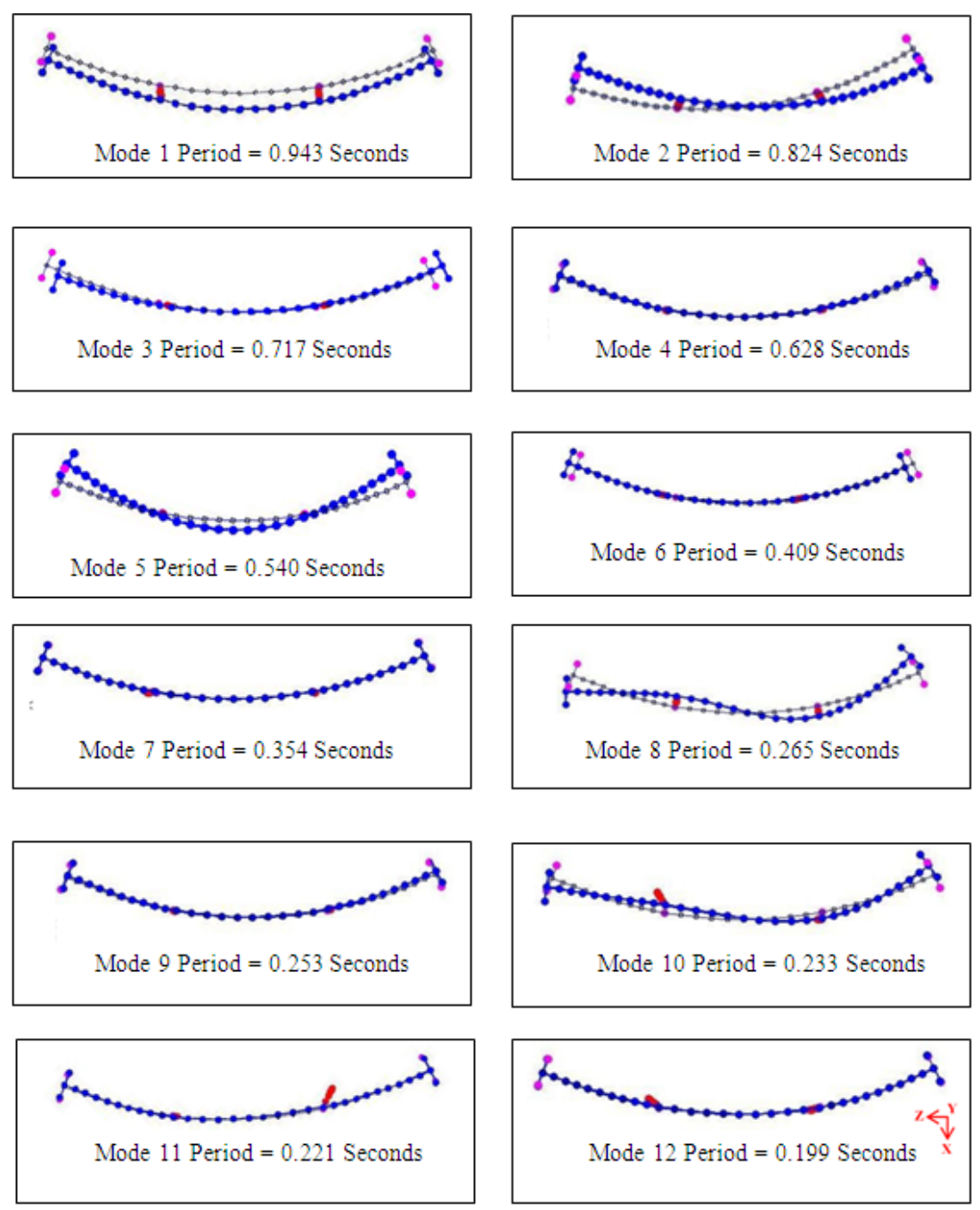

Figure B.31 - Mode Shapes and Period summary for $\theta=45^{\circ}$ (Longitudinal Abutment Stiffness $=0.55 \mathrm{~K}_{\text {eff }}$ )
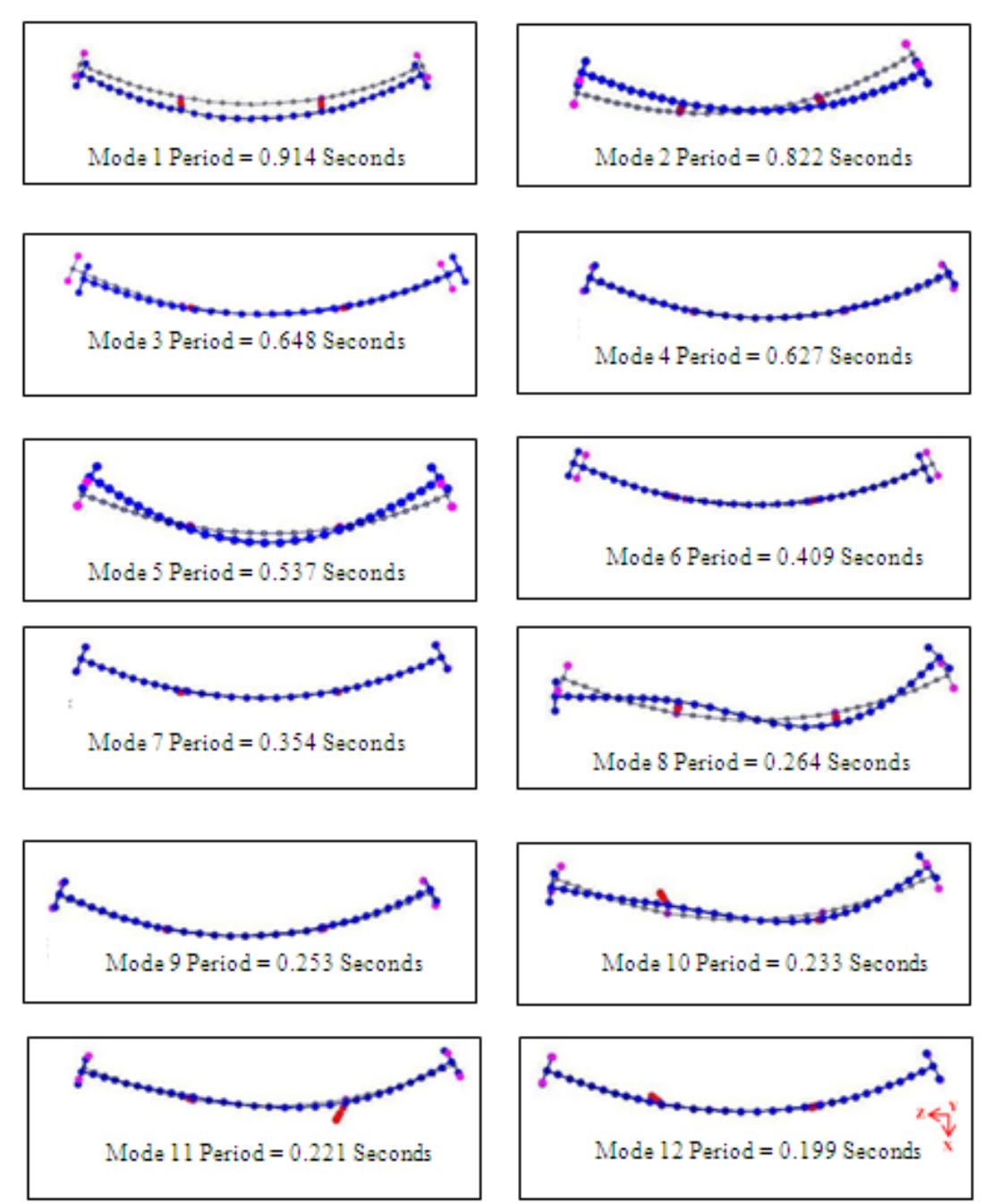

Figure B.32 - Mode Shapes and Period summary for $\theta=45^{\circ}$ (Longitudinal Abutment Stiffness $=1.00 \mathrm{~K}_{\text {eff }}$ ) 


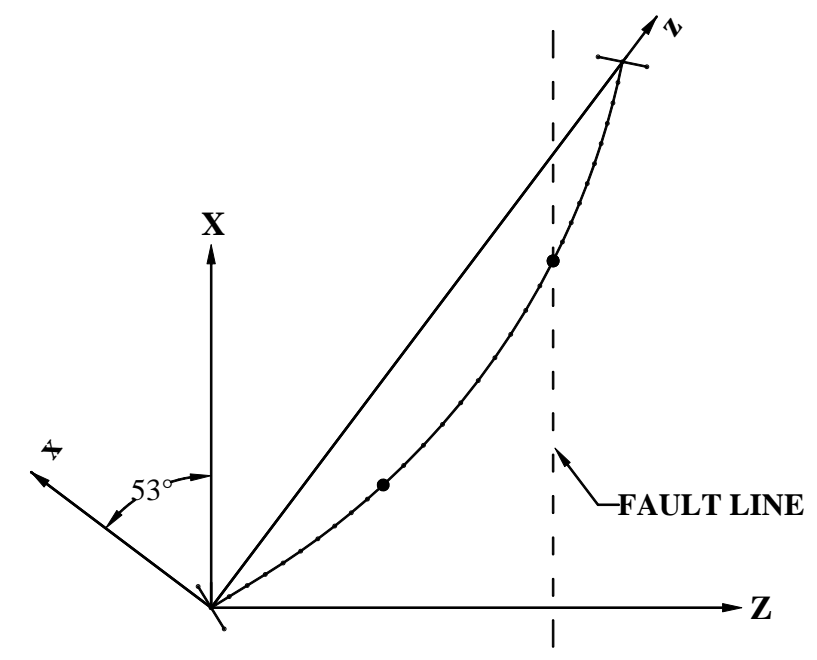

Figure B.33 - Sketch of Bridge 55-0837S when $\theta=53^{\circ}$
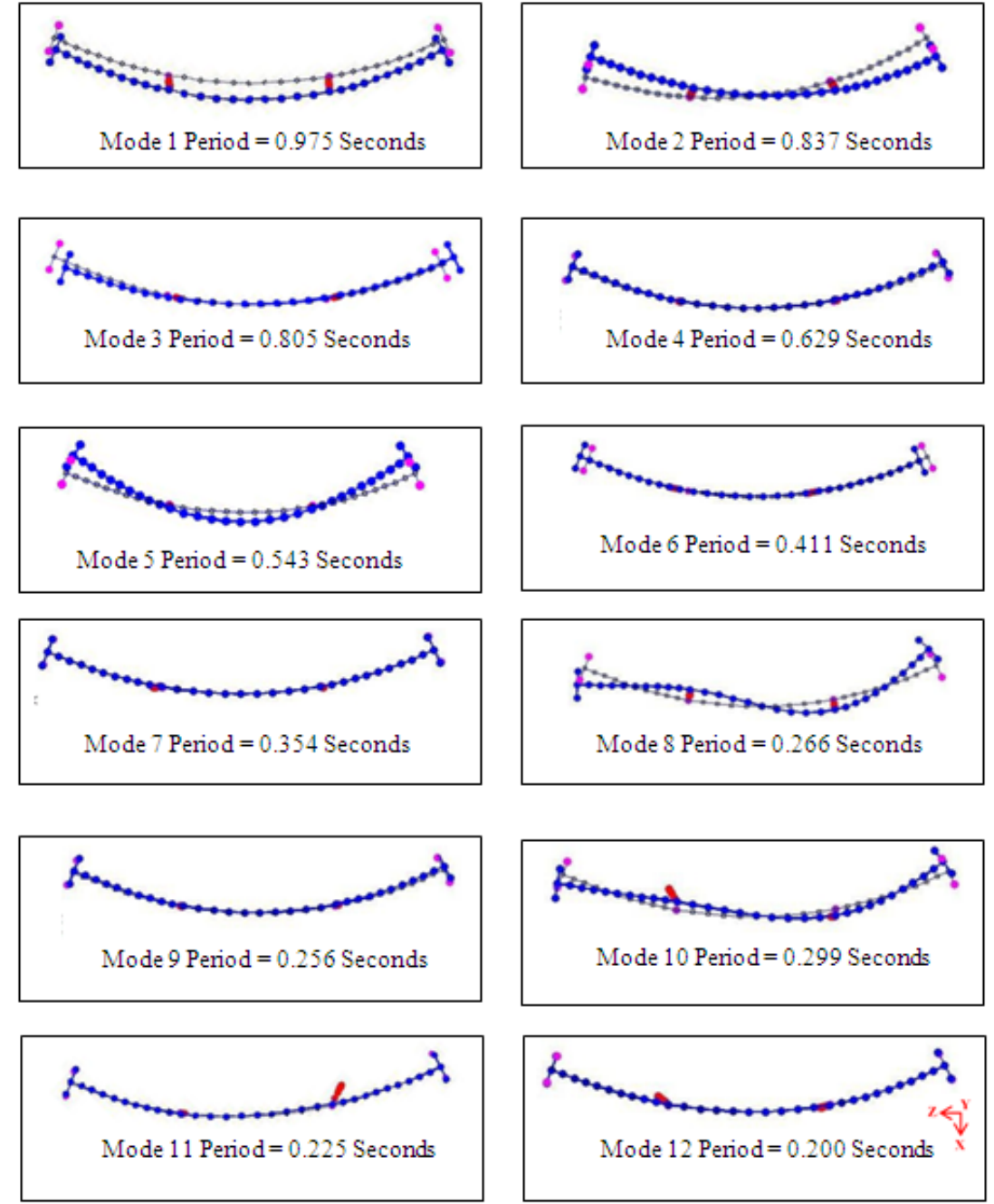

Figure B.34 - Mode Shapes and Period summary for $\theta=53^{\circ}$ (Longitudinal Abutment Stiffness $=0.10 \mathrm{~K}_{\text {eff }}$ ) 

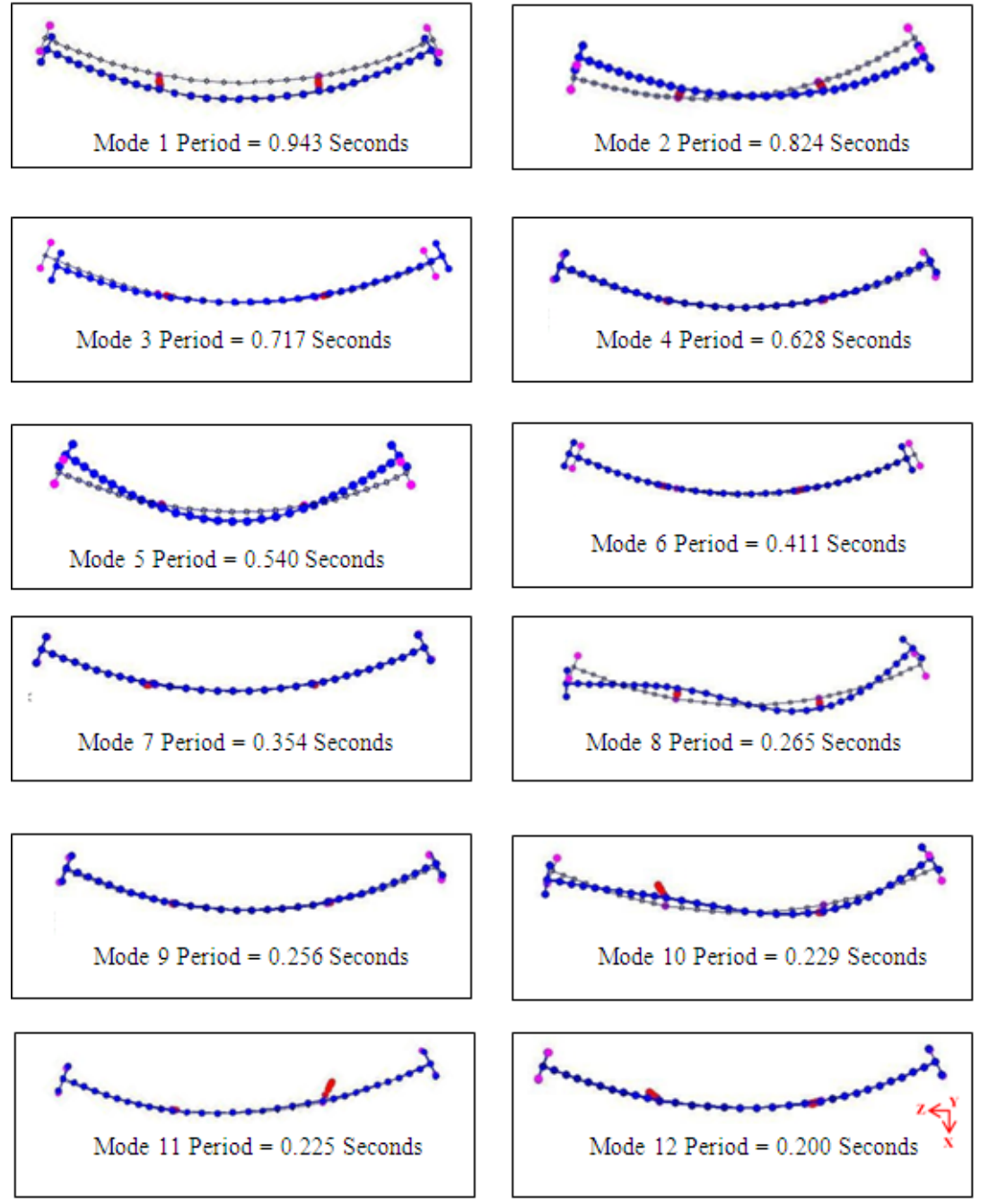

Figure B.35 - Mode Shapes and Period summary for $\theta=53^{\circ}$ (Longitudinal Abutment Stiffness $=0.55 \mathrm{~K}_{\text {eff }}$ )
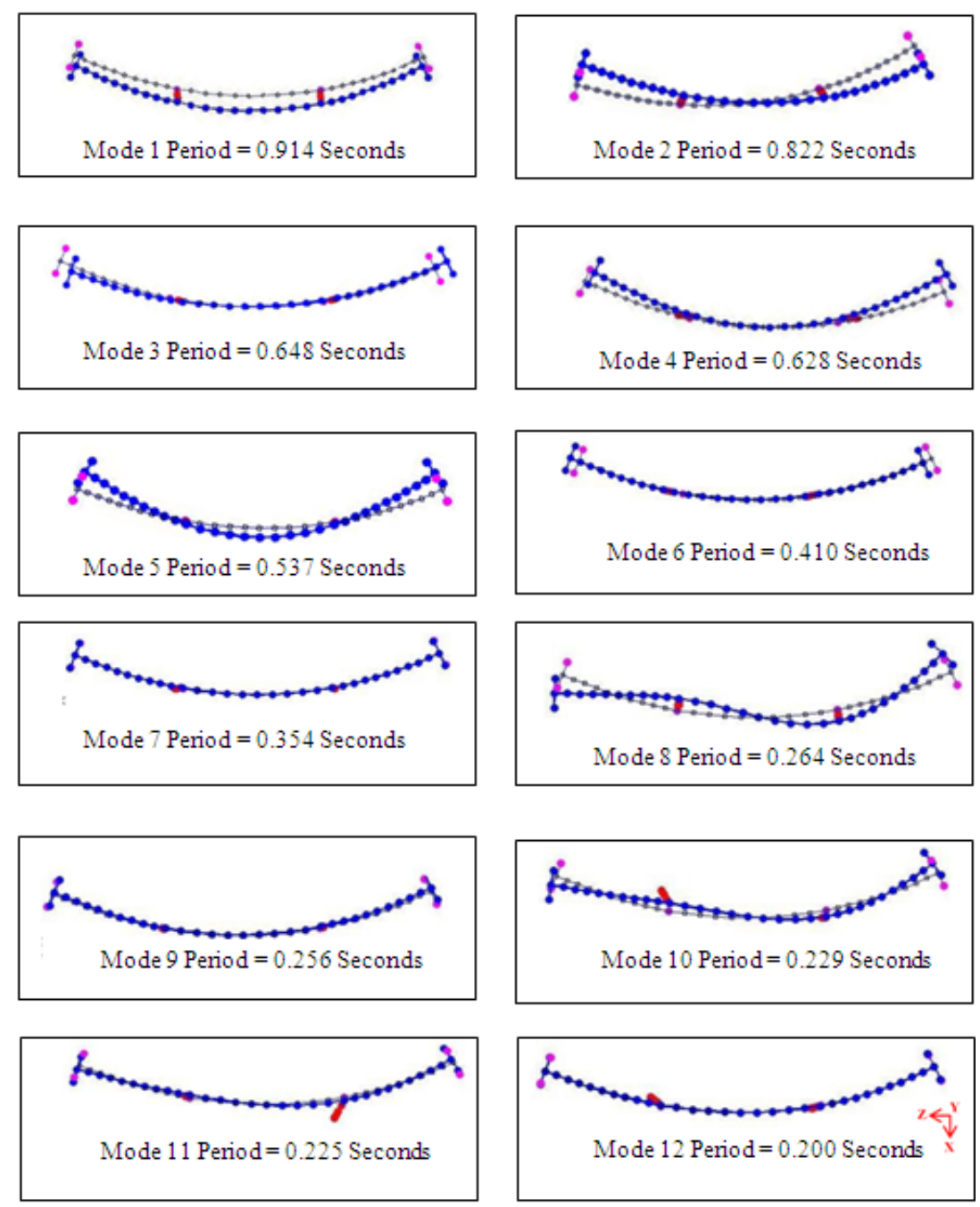

Figure B.36 - Mode Shapes and Period summary for $\theta=53^{\circ}$ (Longitudinal Abutment Stiffness $\left.=1.00 \mathrm{~K}_{\text {eff }}\right)$ 
Appendix C : Complete Result Comparisons of Bridge 55-0837S 


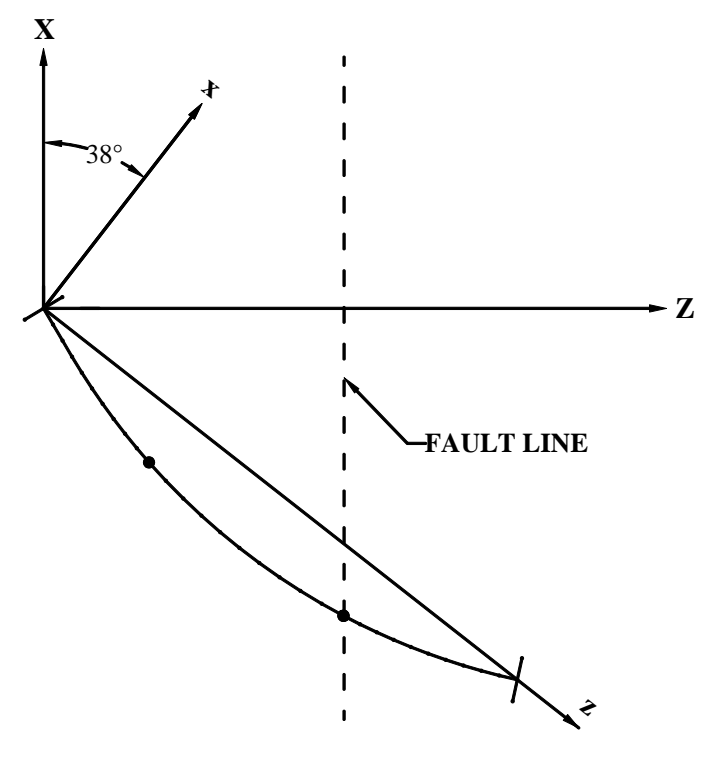

Figure C.1 - Sketch of Bridge 55-0837S when $\theta=-38^{\circ}$
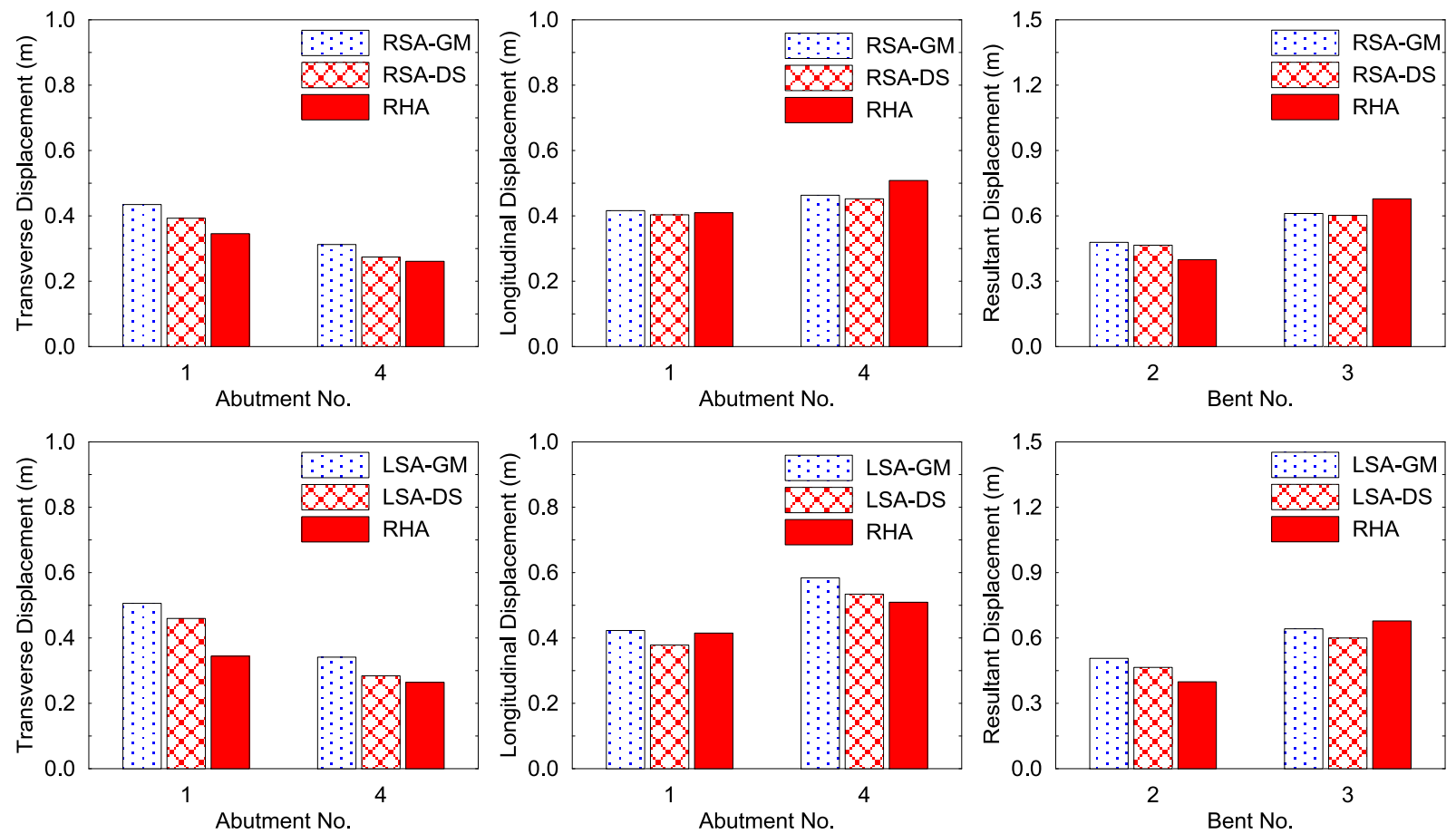

Figure C. 2 - Results of Bridge 55-0837S when $\theta=-38^{\circ}$ and longitudinal abutment stiffness $=0.10 K_{\text {eff }}$. 

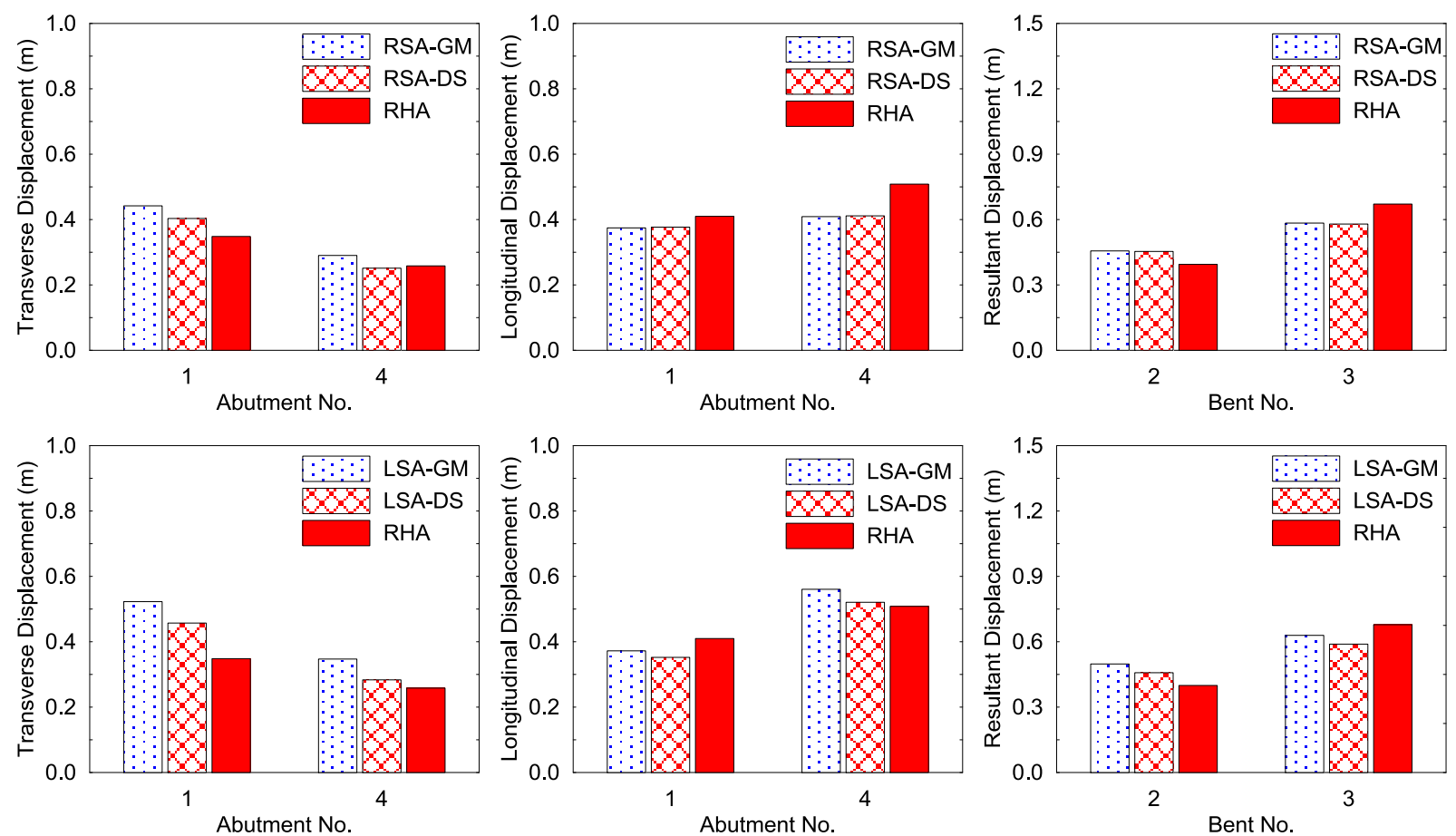

Figure C. 3 - Results of Bridge 55-0837S when $\theta=-38^{\circ}$ and longitudinal abutment stiffness $=0.55 K_{\text {eff }}$
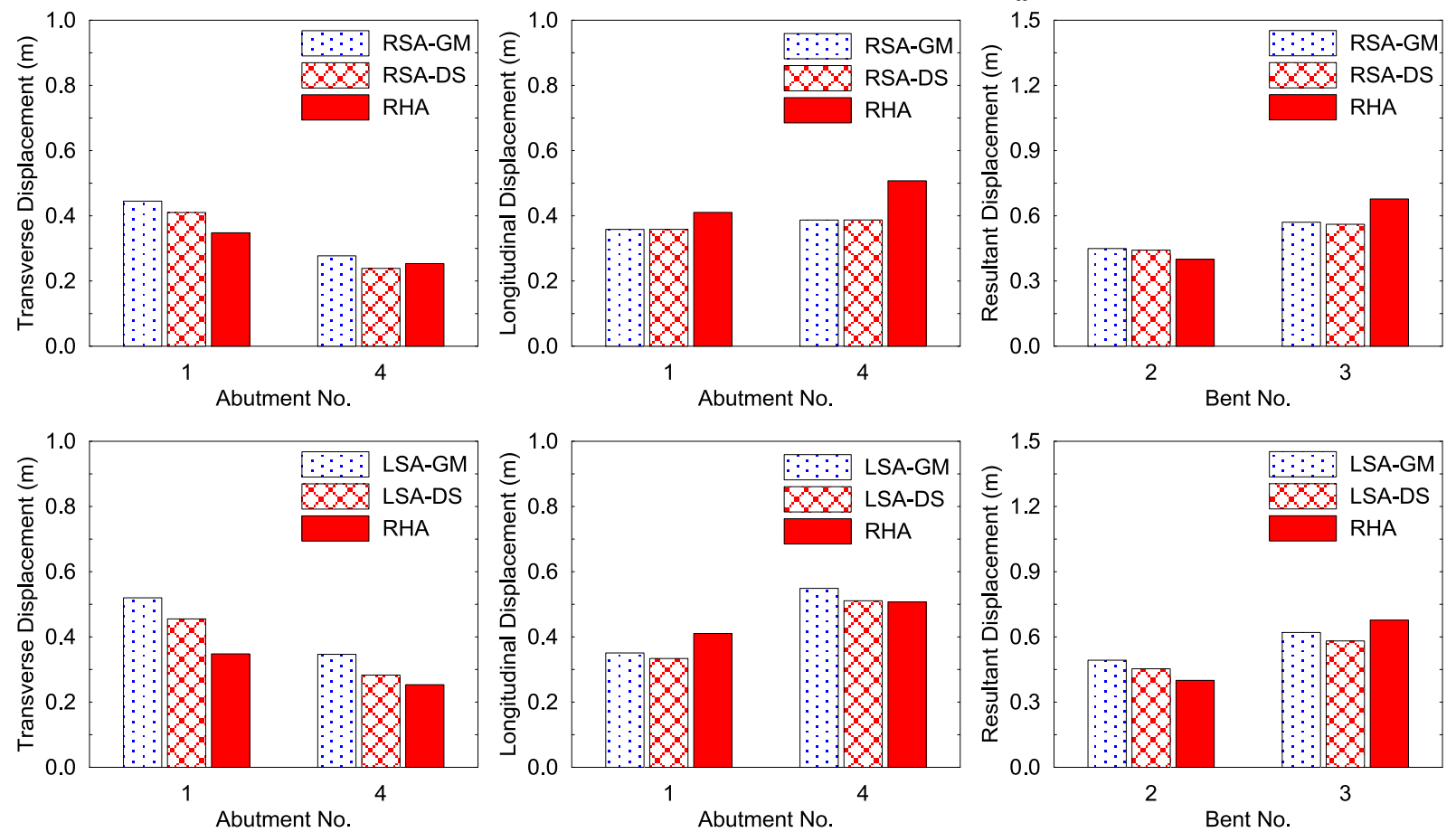

Figure C. 4 - Results of Bridge 55-0837S when $\theta=-38^{\circ}$ and longitudinal abutment stiffness $=1.00 K_{\text {eff }}$. 


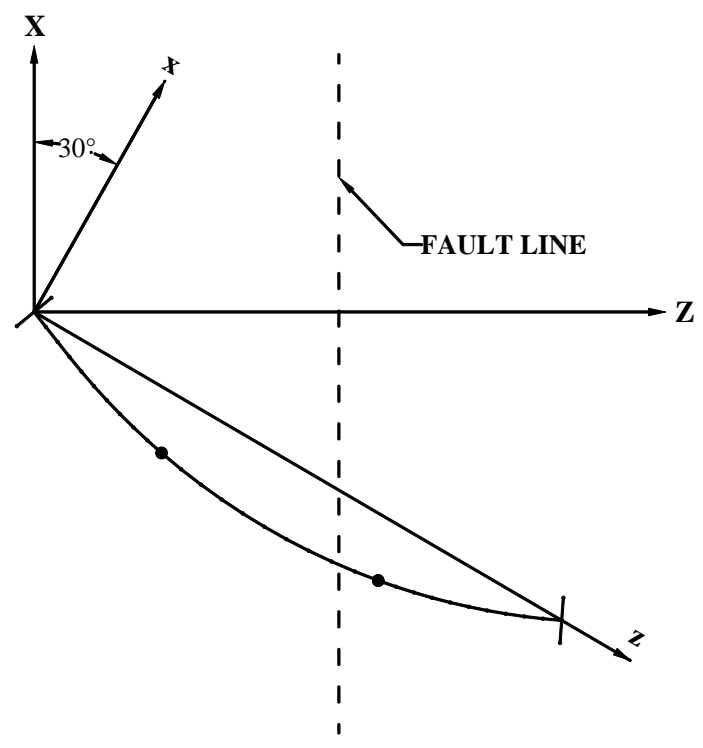

Figure C.5 - Sketch of Bridge 55-0837S when $\theta=-30^{\circ}$
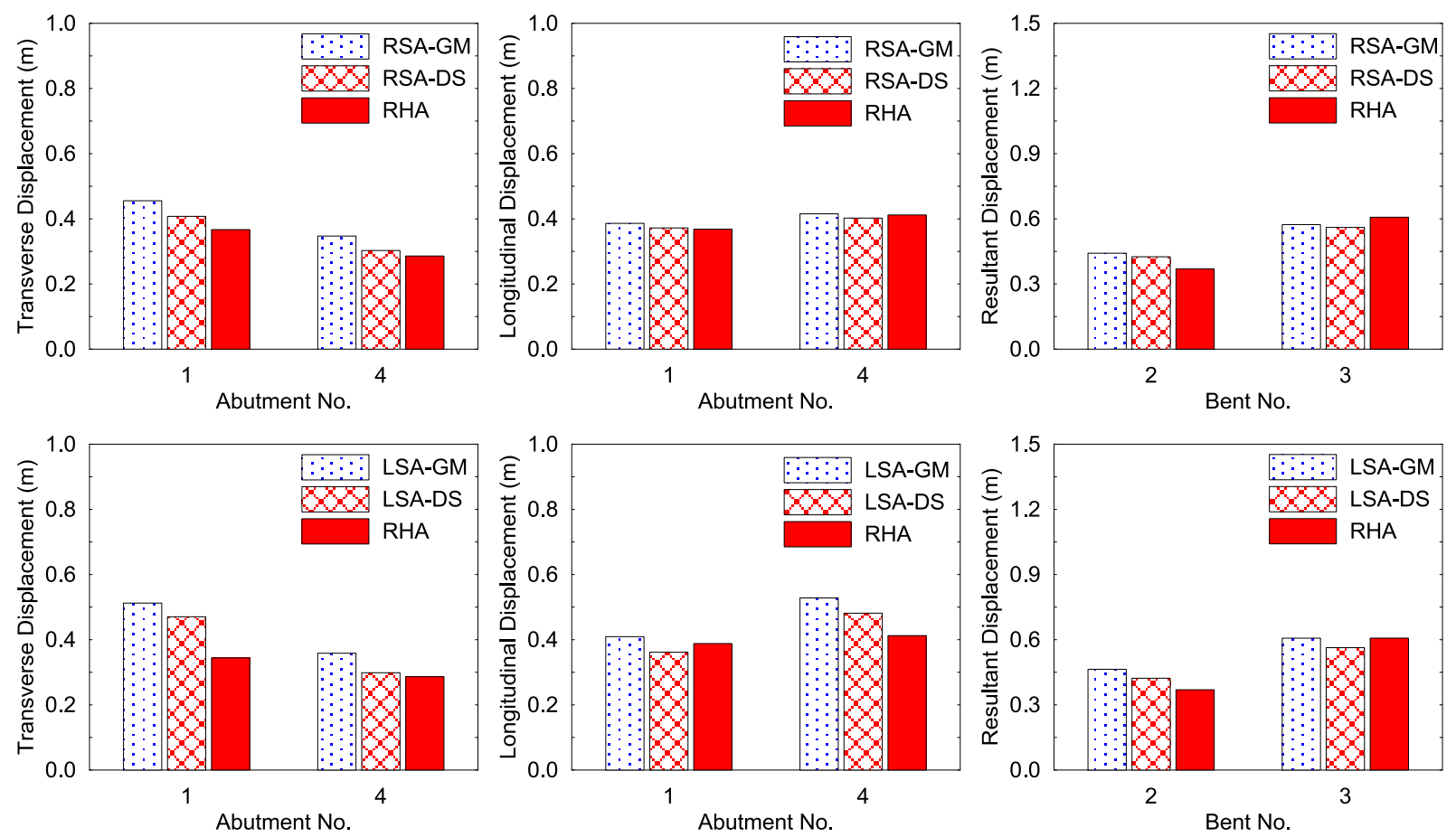

Figure C.6 - Results of Bridge 55-0837S when $\theta=-30^{\circ}$ and longitudinal abutment stiffness $=0.10 K_{\text {eff }}$ 

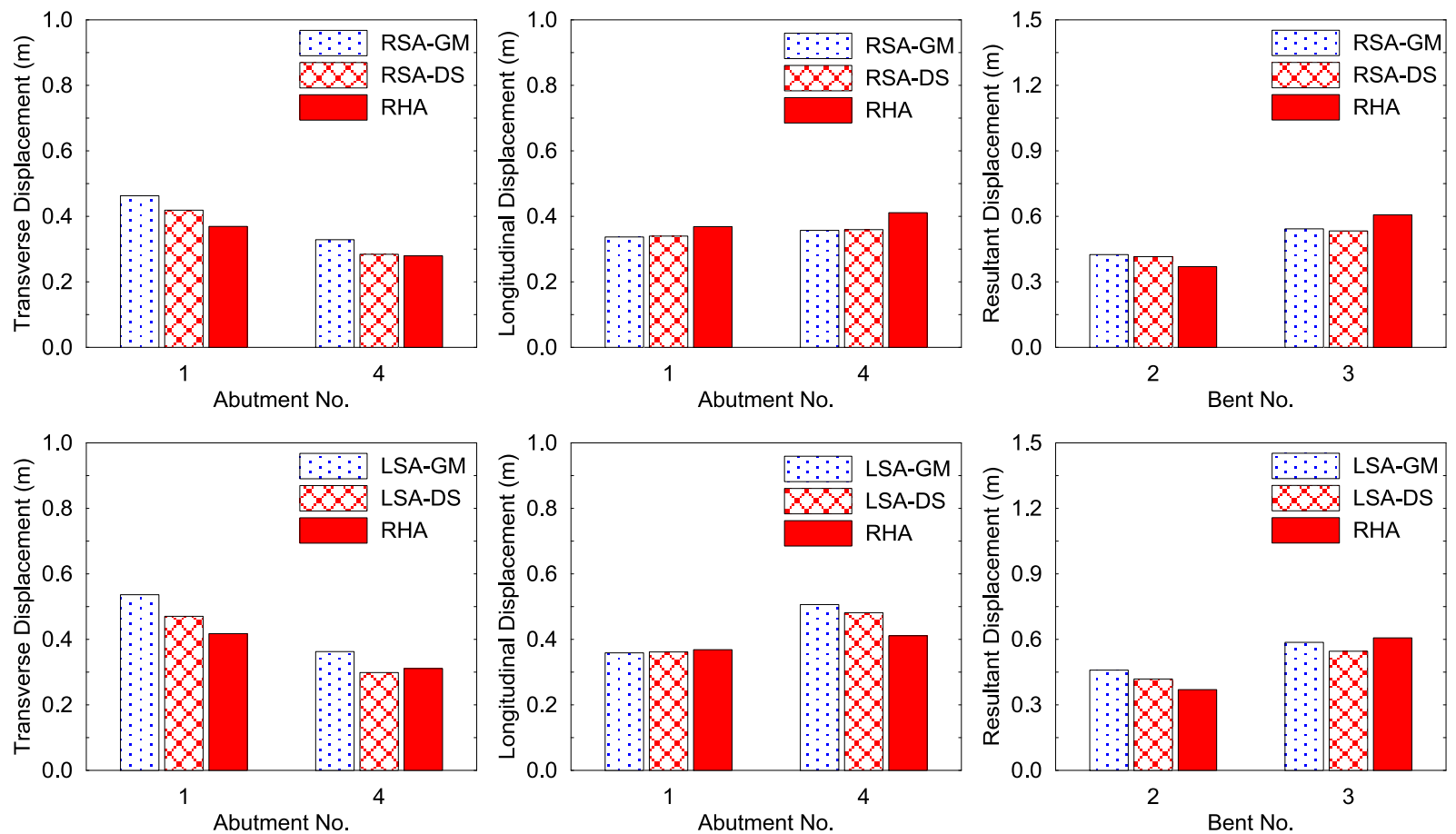

Figure C.7 - Results of Bridge 55-0837S when $\theta=-30^{\circ}$ and longitudinal abutment stiffness $=0.55 K_{\text {eff }}$
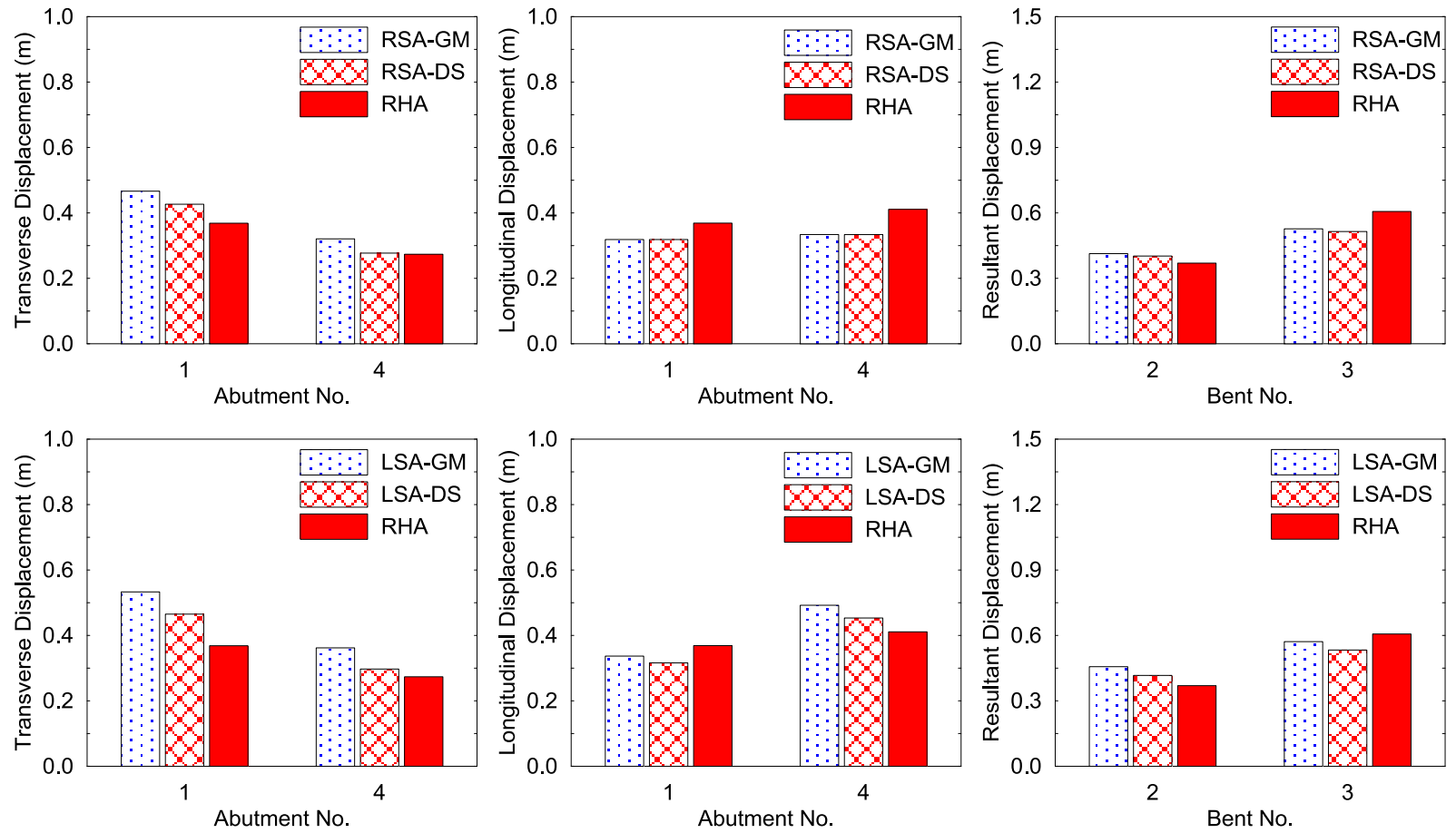

Figure C. 8 - Results of Bridge 55-0837S when $\theta=-30^{\circ}$ and longitudinal abutment stiffness $=1.00 K_{\text {eff }}$ 


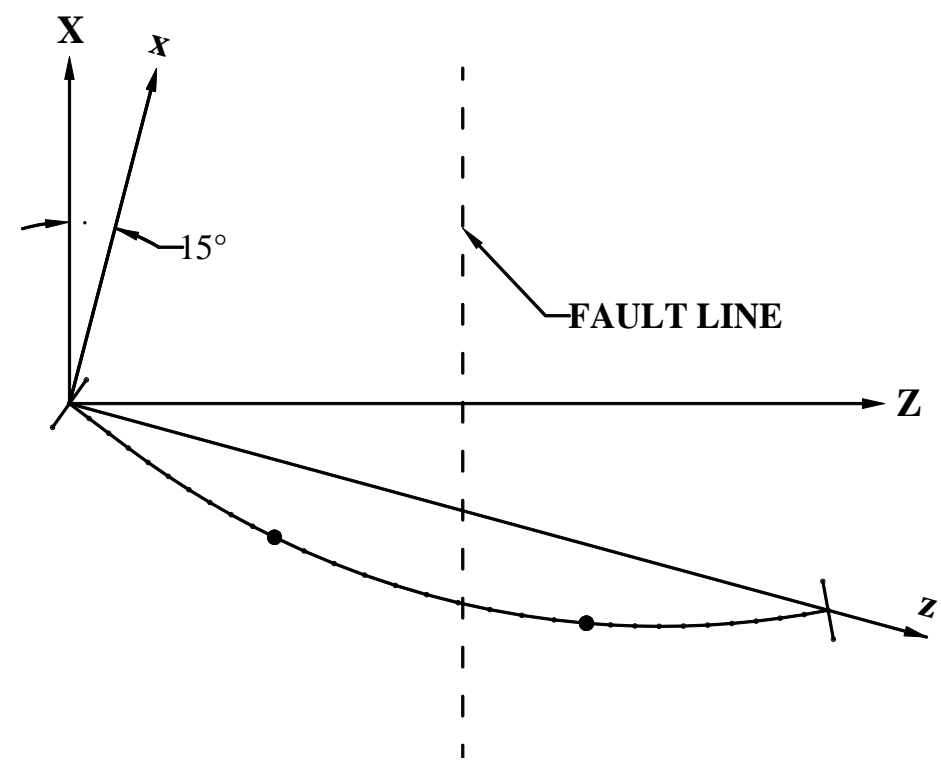

Figure C.9 - Sketch of Bridge 55-0837S when $\theta=-15^{\circ}$
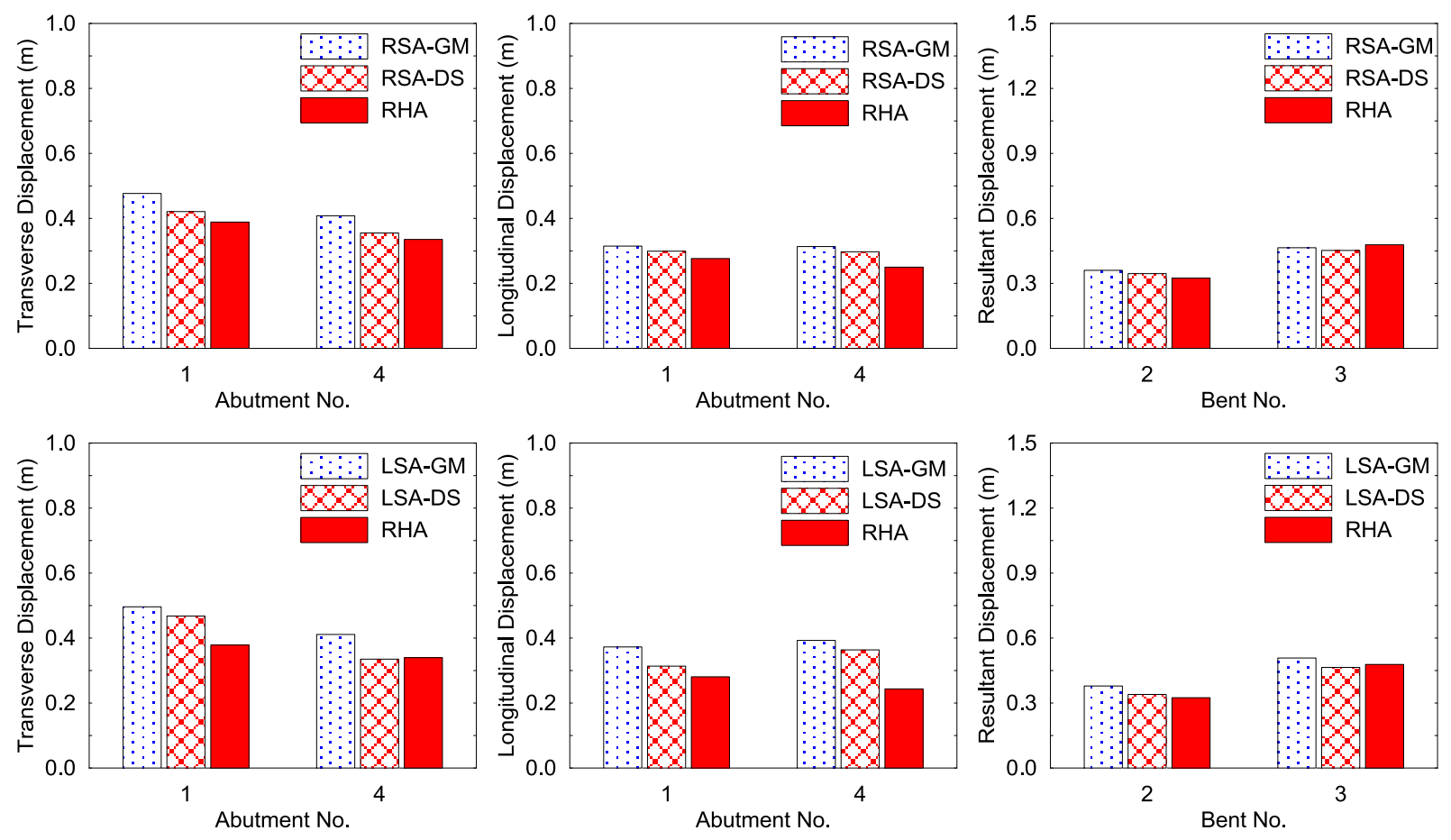

Figure C.10 - Results of Bridge 55-0837S when $\theta=-15^{\circ}$ and longitudinal abutment stiffness $=0.10 K_{\text {eff }}$ 

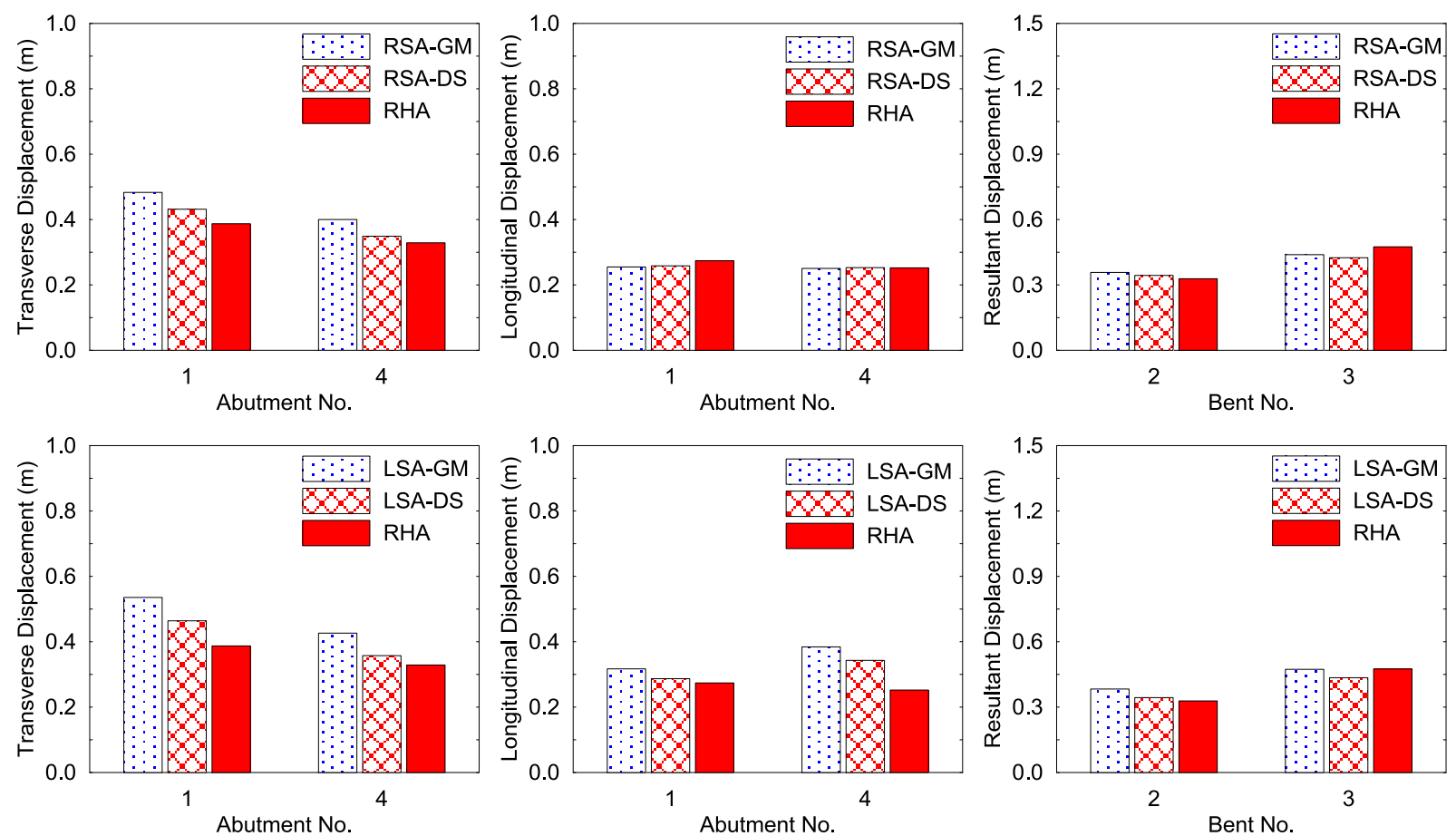

Figure C.11 - Results of Bridge 55-0837S when $\theta=-15^{\circ}$ and longitudinal abutment stiffness $=0.55 K_{\text {eff }}$
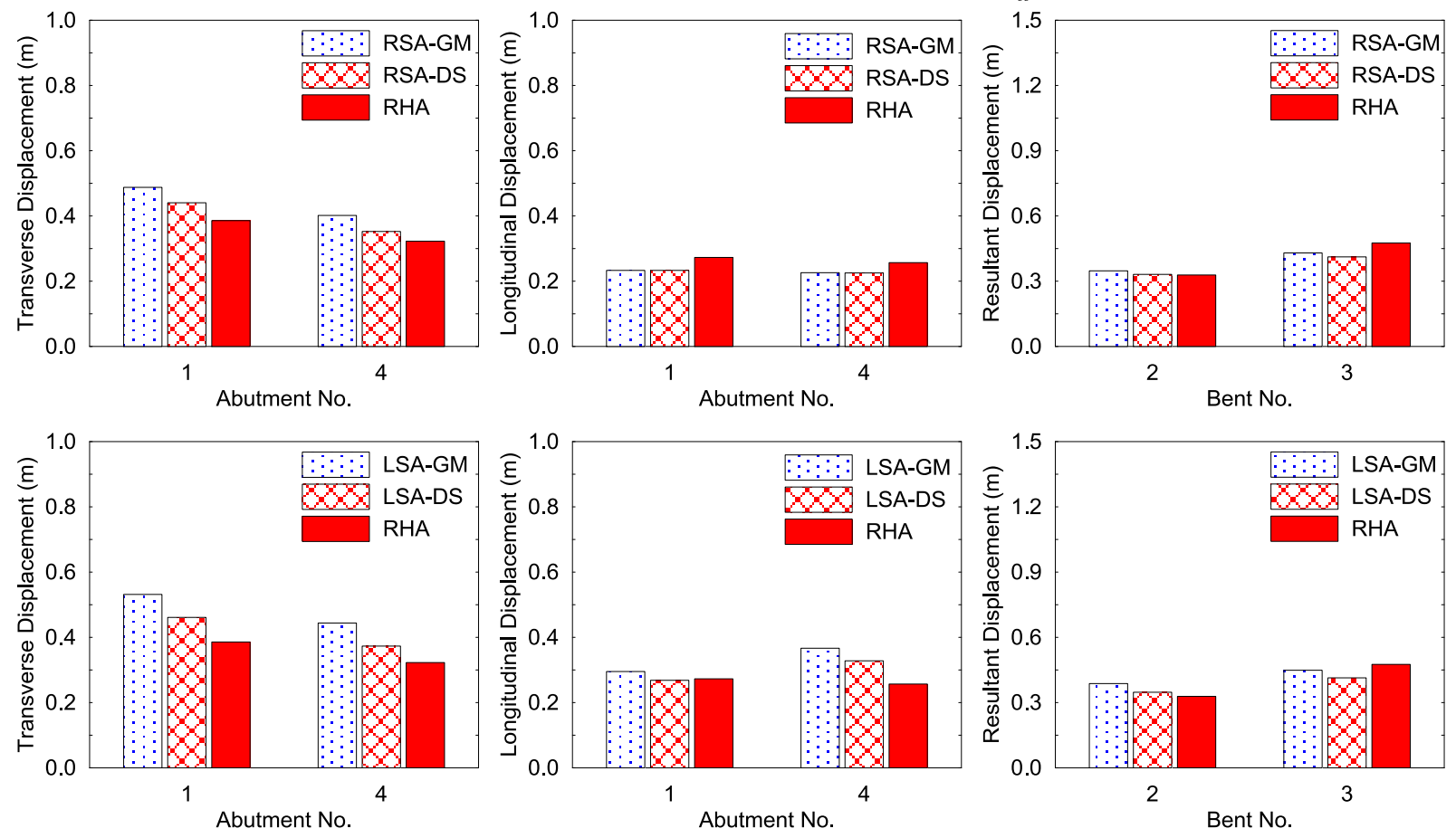

Figure C.12 - Results of Bridge 55-0837S when $\theta=-15^{\circ}$ and longitudinal abutment stiffness $=1.00 K_{\text {eff }}$ 


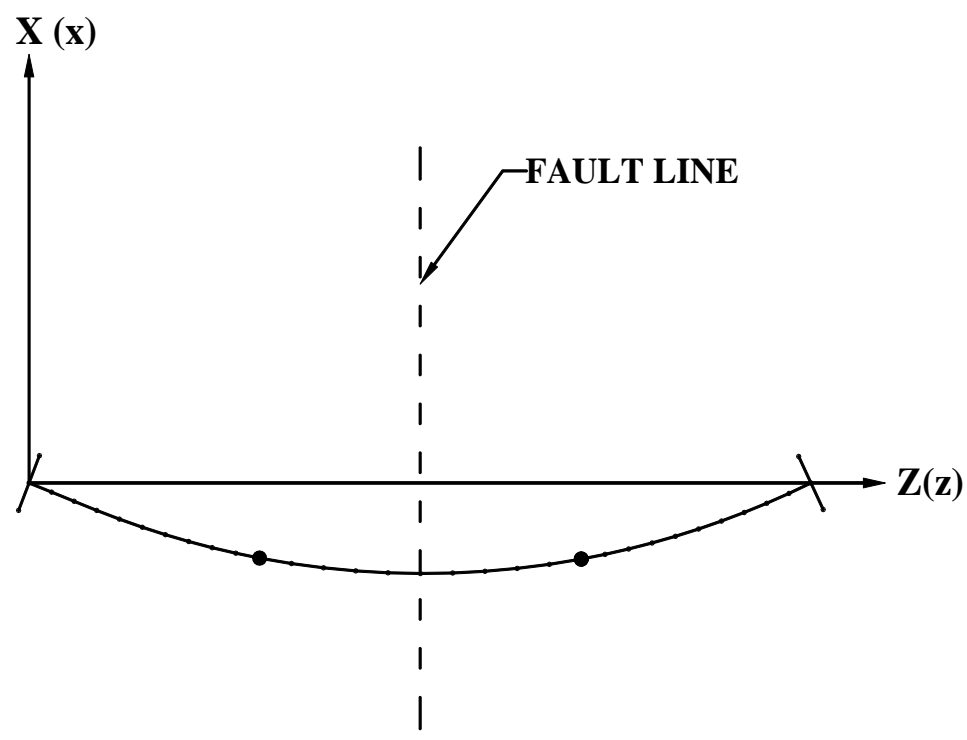

Figure C.13 - Sketch of Bridge 55-0837S when $\theta=0^{\circ}$
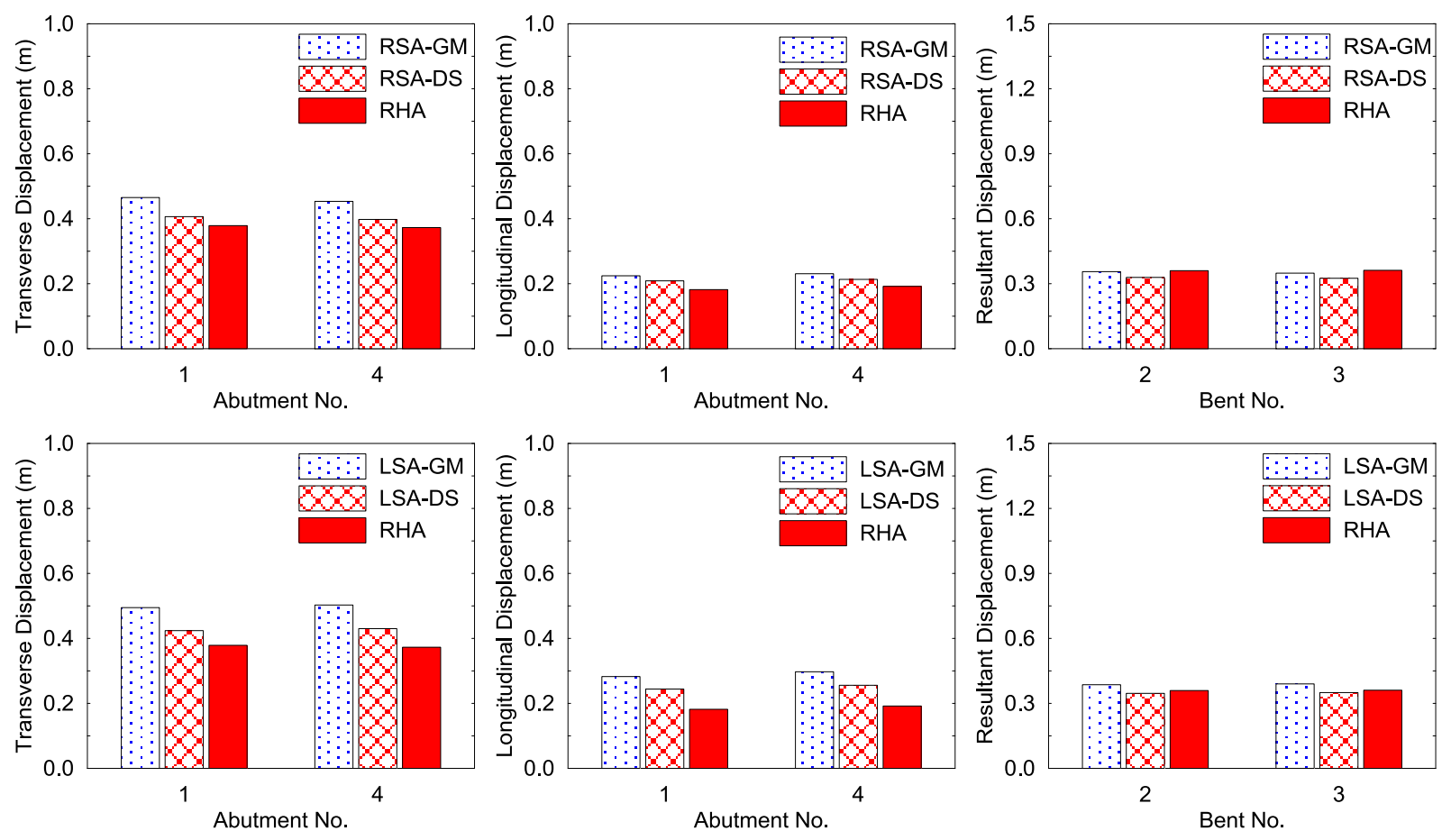

Figure C.14 - Results of Bridge 55-0837S when $\theta=0^{\circ}$ and longitudinal abutment stiffness $=0.10 K_{\text {eff }}$ 

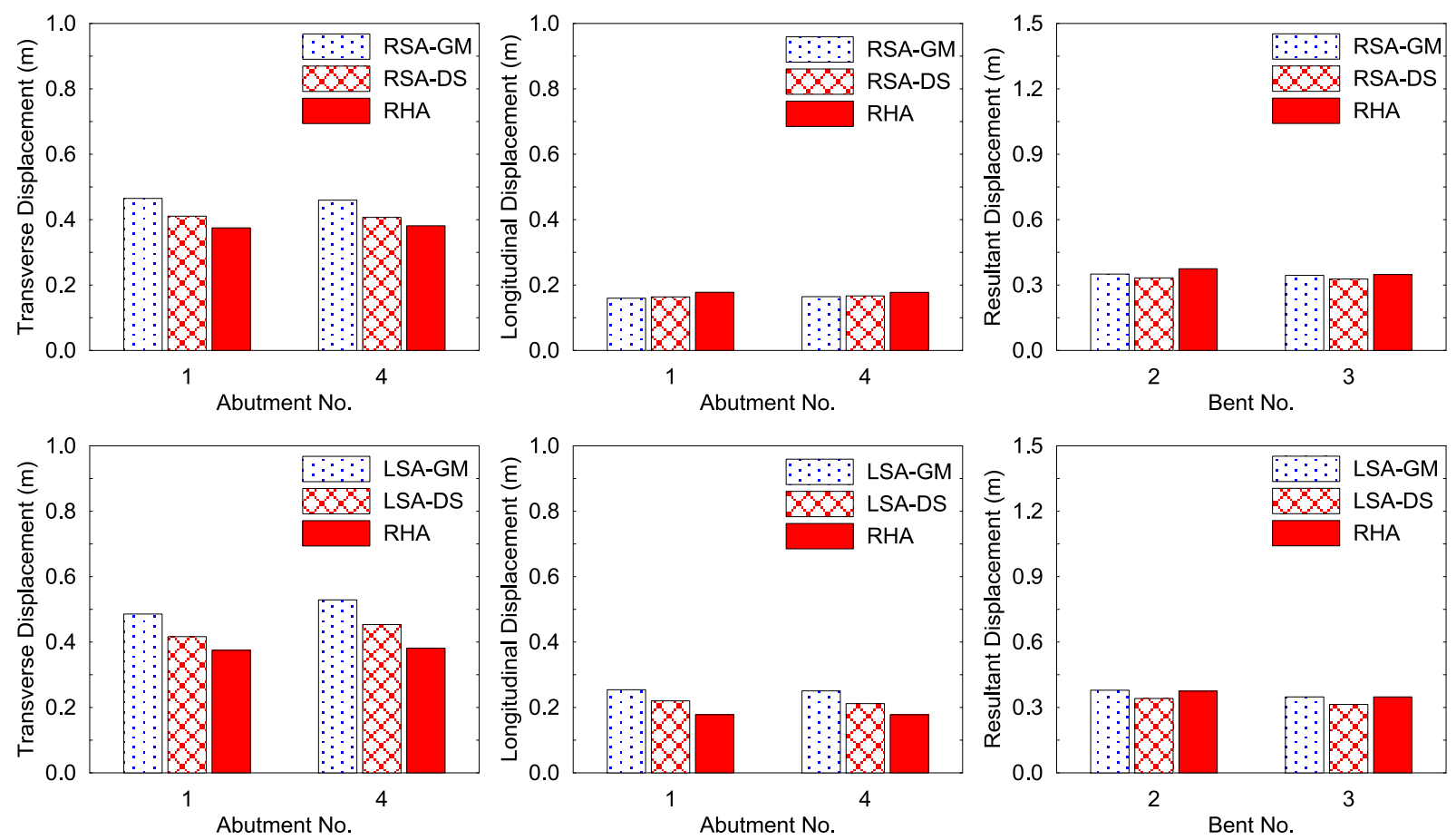

Figure C.15 - Results of Bridge 55-0837S when $\theta=0^{\circ}$ and longitudinal abutment stiffness $=0.55 K_{\text {eff }}$
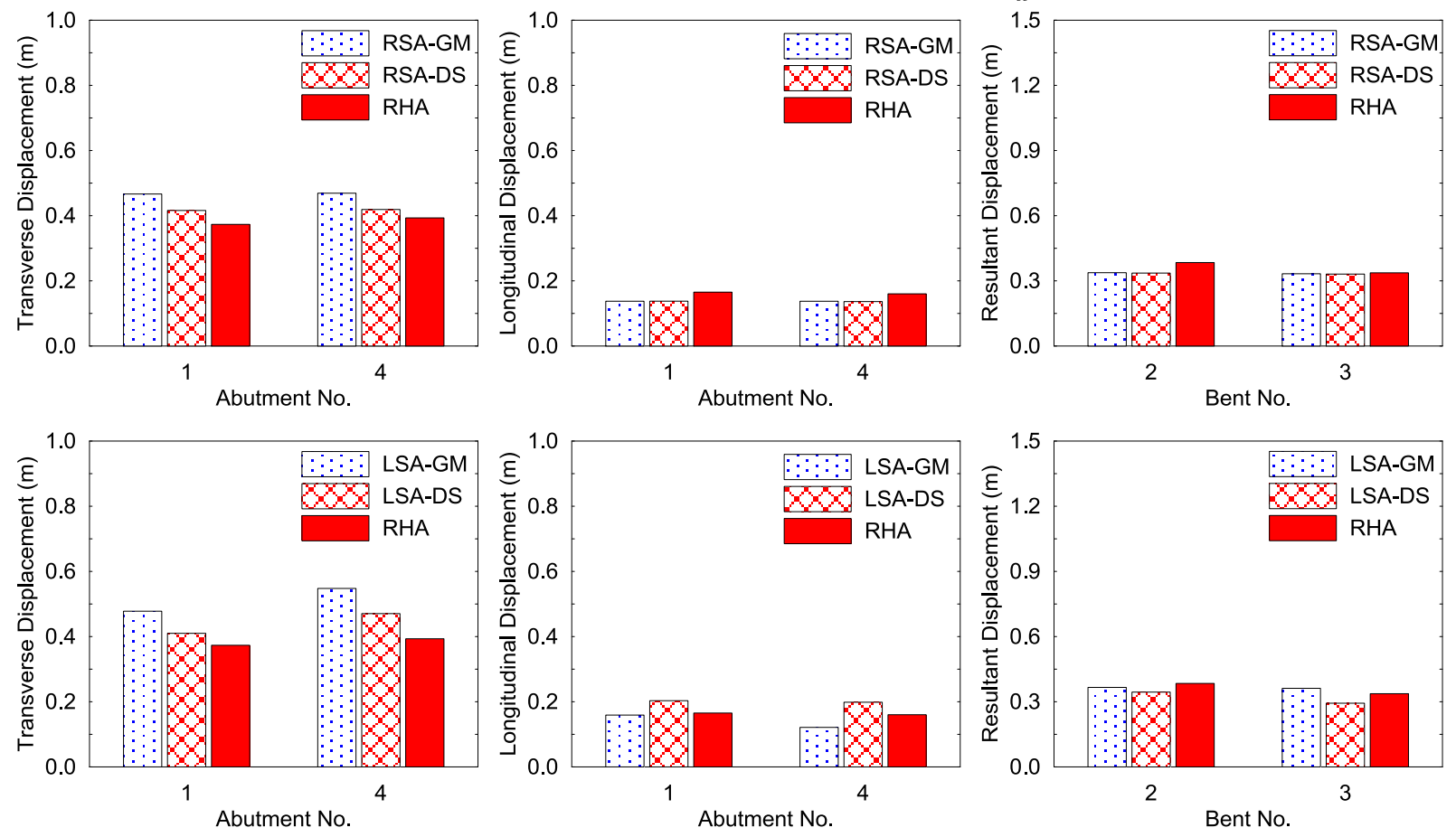

Figure C.16 - Results of Bridge 55-0837S when $\theta=0^{\circ}$ and longitudinal abutment stiffness $=1.00 K_{\text {eff }}$ 


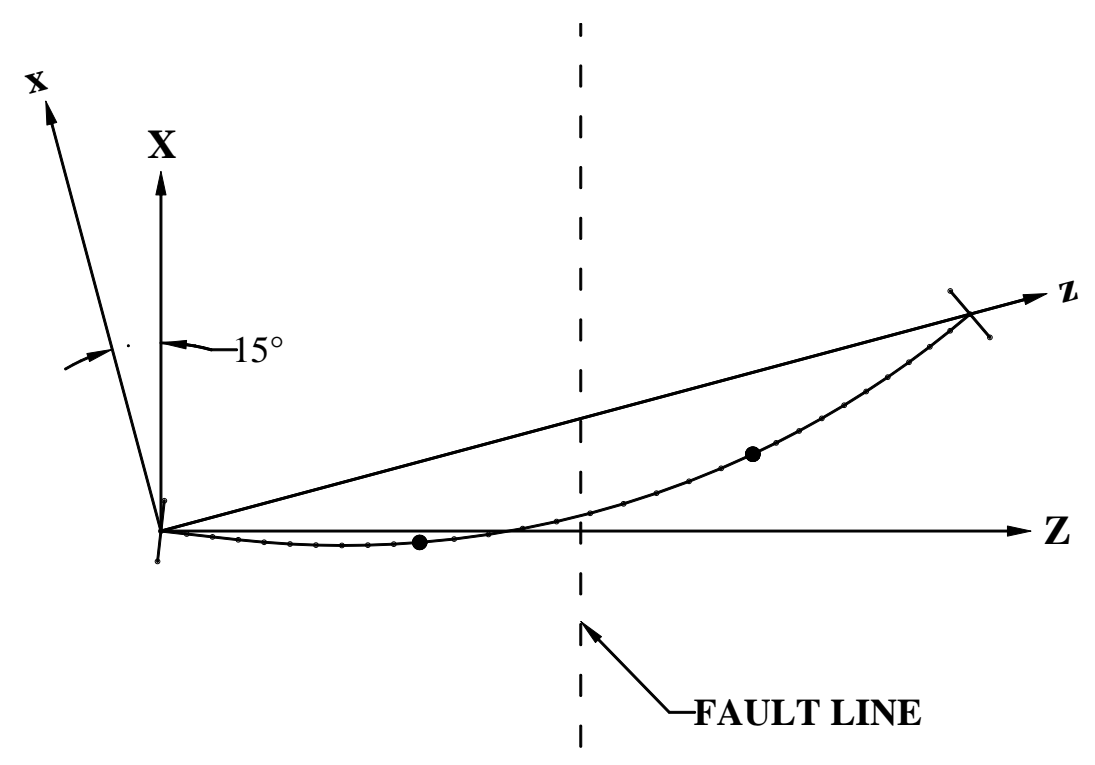

Figure C. 17 - Sketch of Bridge 55-0837S when $\theta=15^{\circ}$
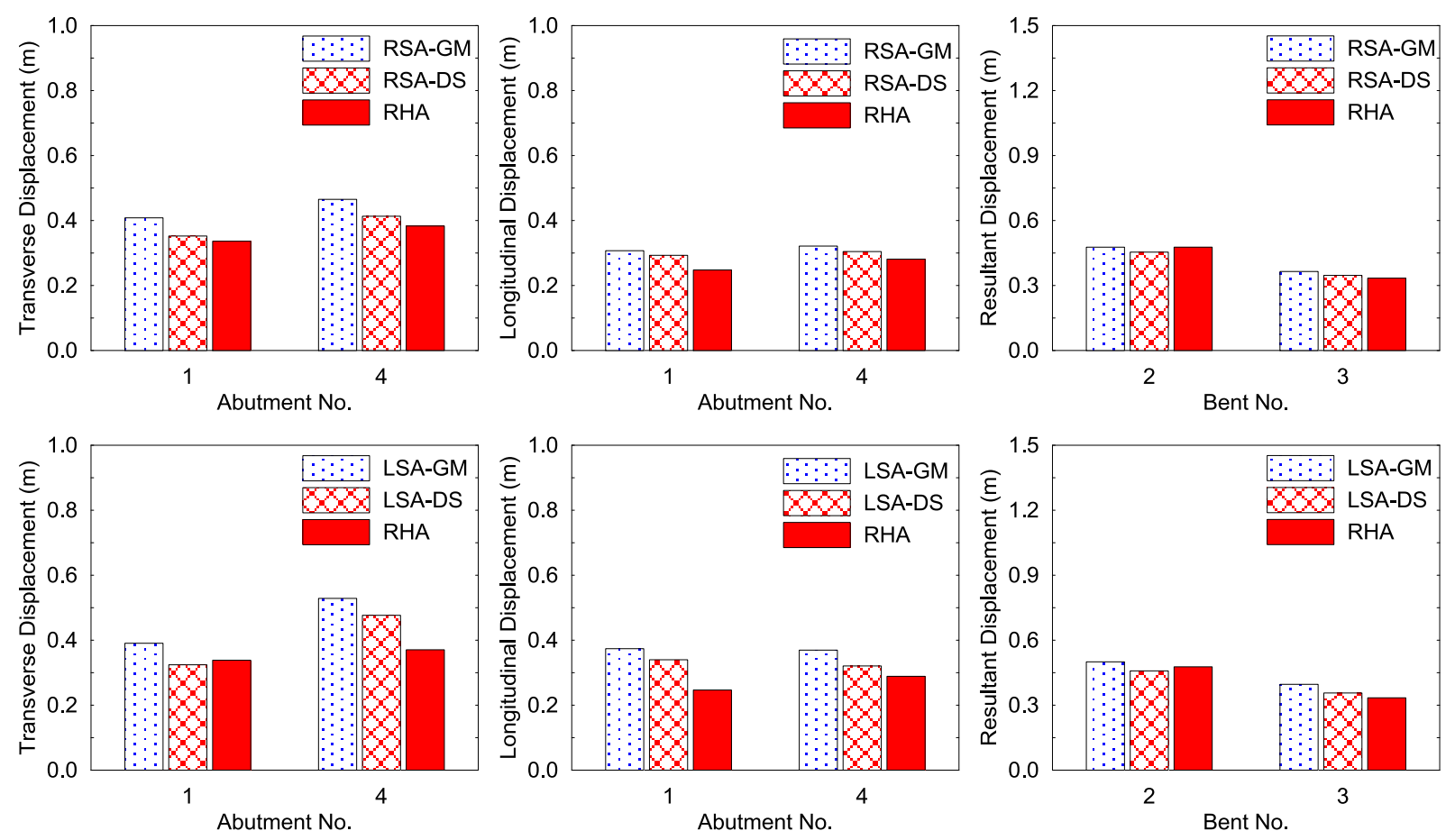

Figure C.18 - Results of Bridge 55-0837S when $\theta=15^{\circ}$ and longitudinal abutment stiffness $=0.10 K_{\text {eff }}$ 

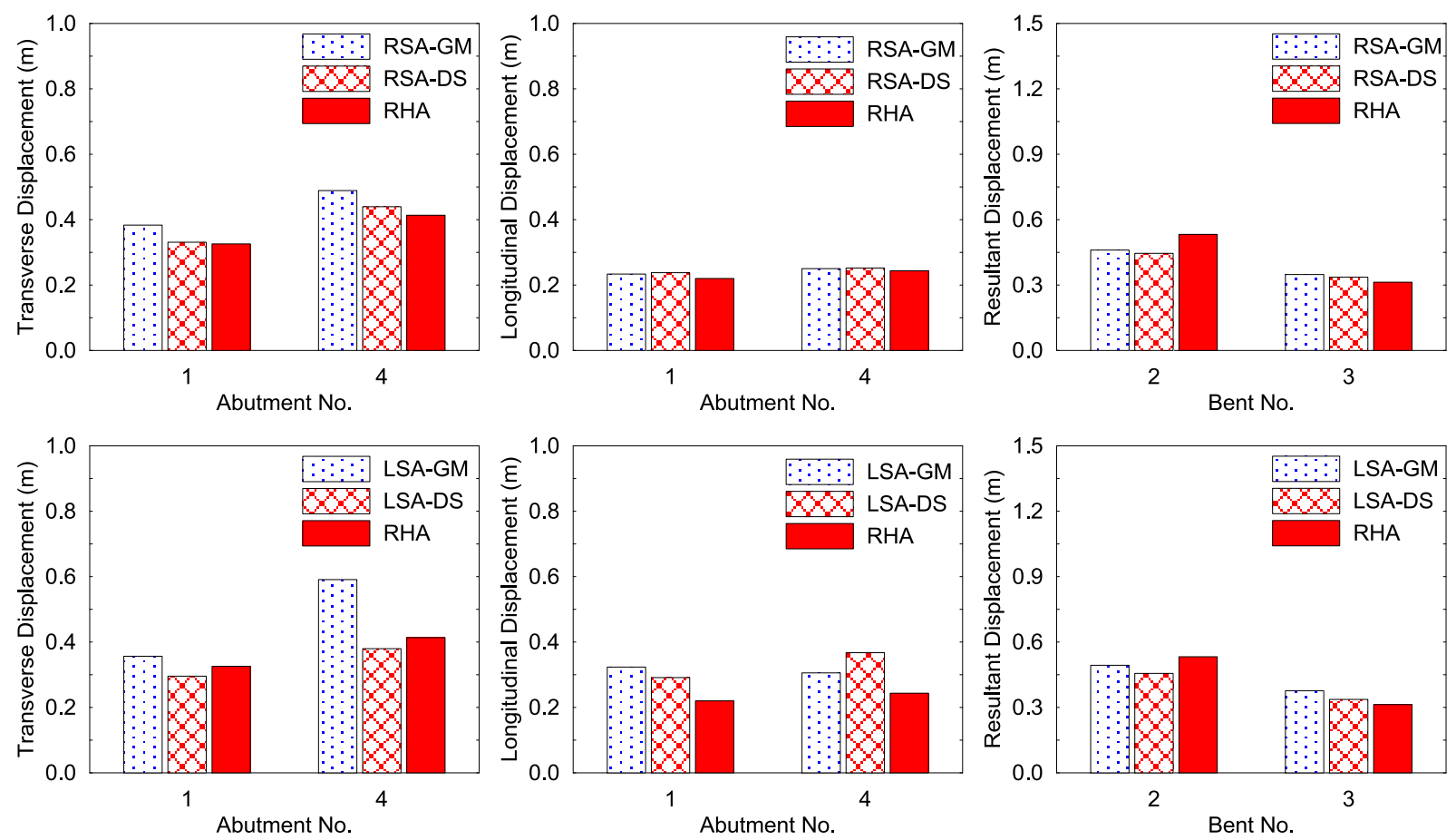

Figure C.19 - Results of Bridge 55-0837S when $\theta=15^{\circ}$ and longitudinal abutment stiffness $=0.55 K_{\text {eff }}$
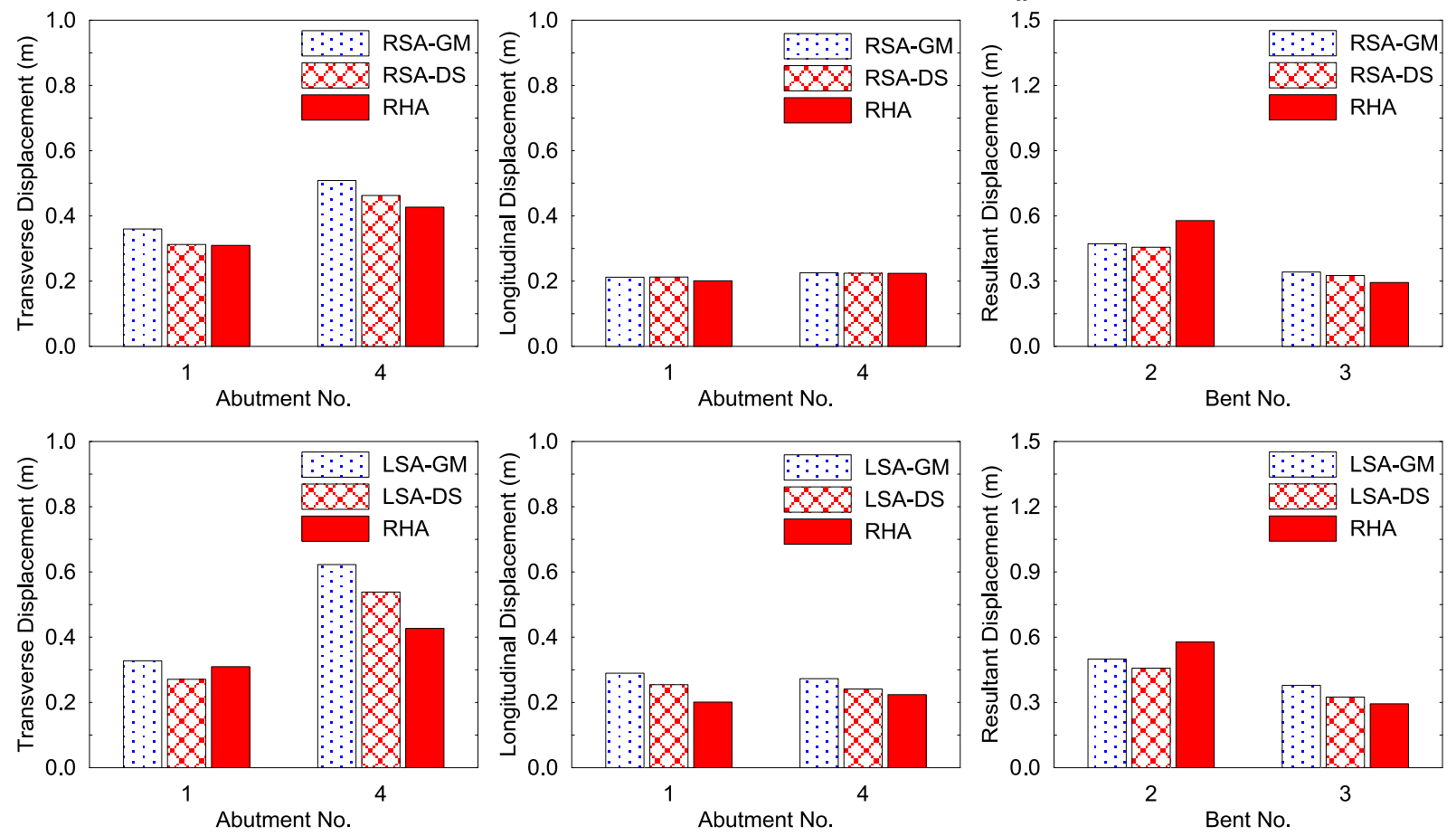

Figure C.20 - Results of Bridge 55-0837S when $\theta=15^{\circ}$ and longitudinal abutment stiffness $=1.00 K_{\text {eff }}$ 


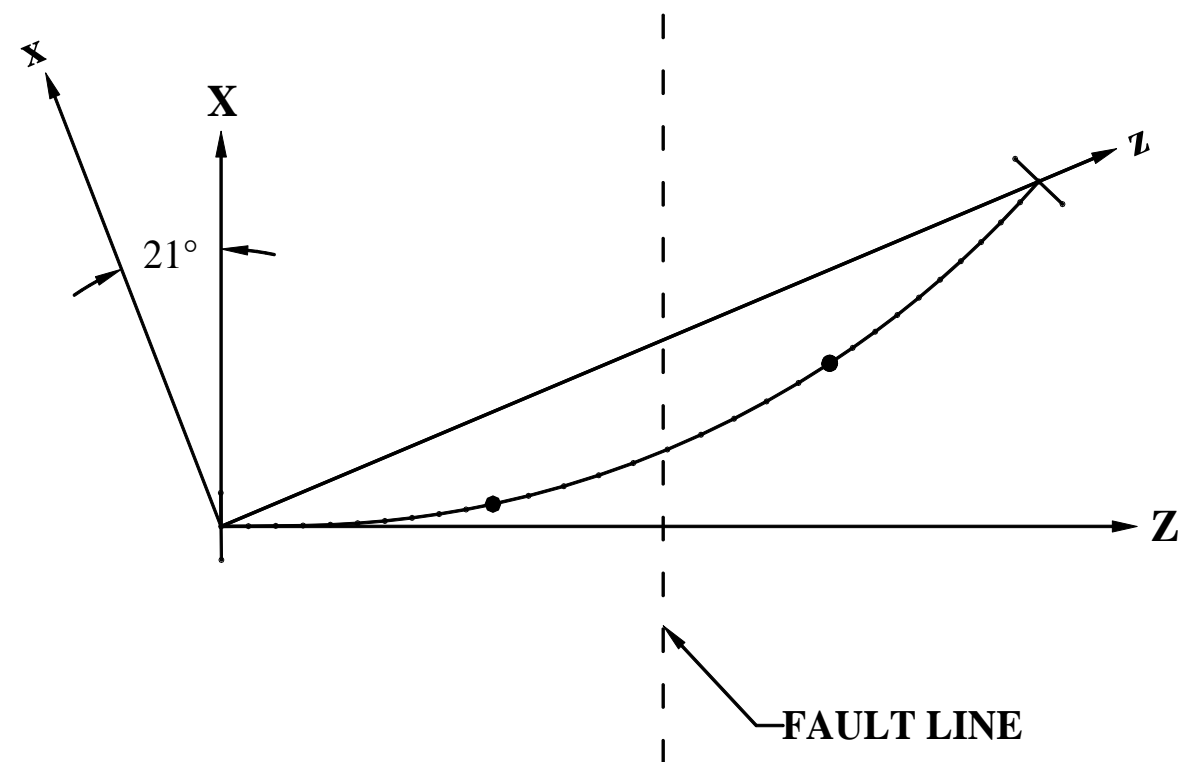

Figure C.21 - Sketch of Bridge 55-0837S when $\theta=21^{\circ}$
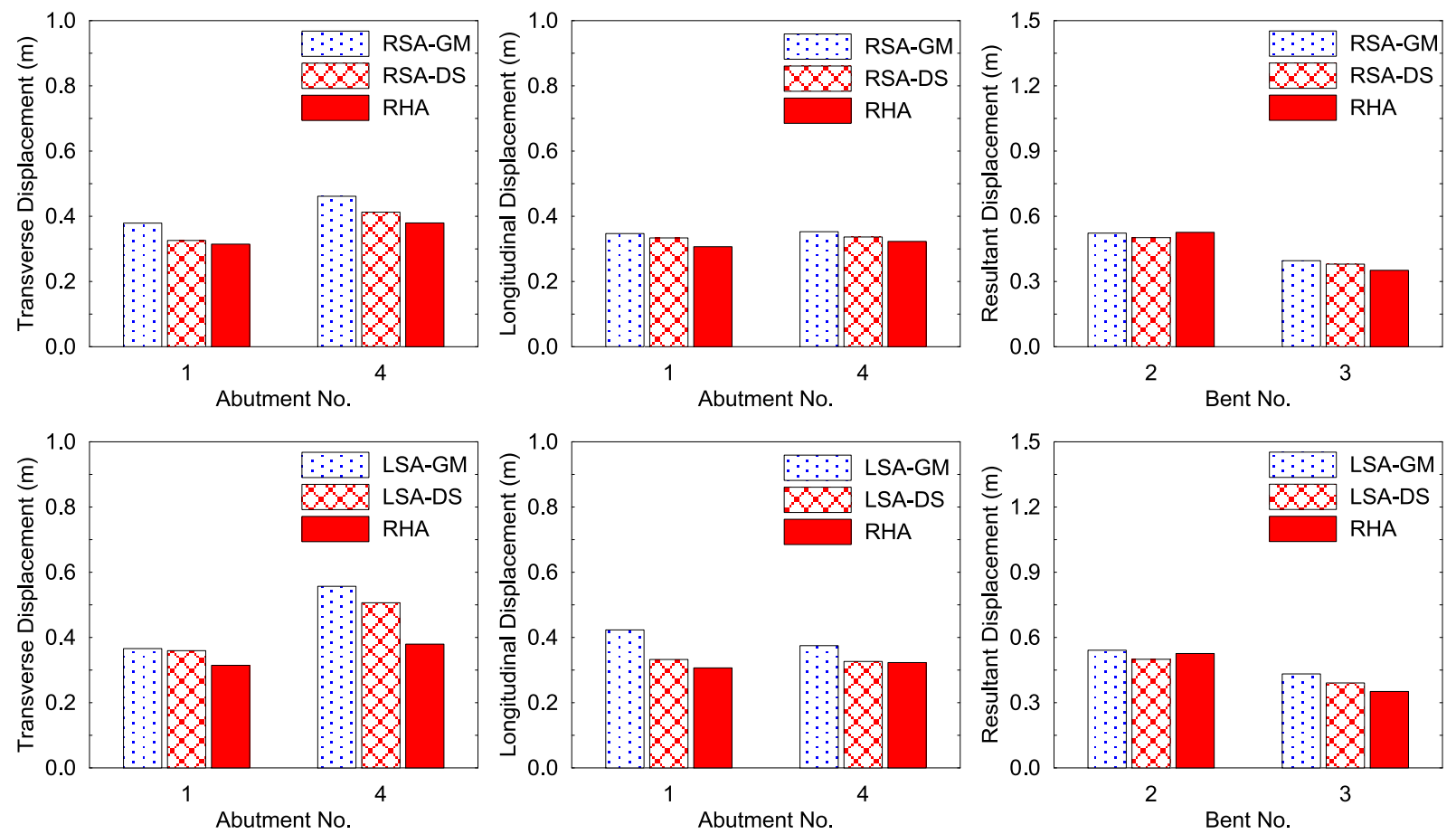

Figure C.22 - Results of Bridge 55-0837S when $\theta=21^{\circ}$ and longitudinal abutment stiffness $=0.10 K_{\text {eff }}$. 

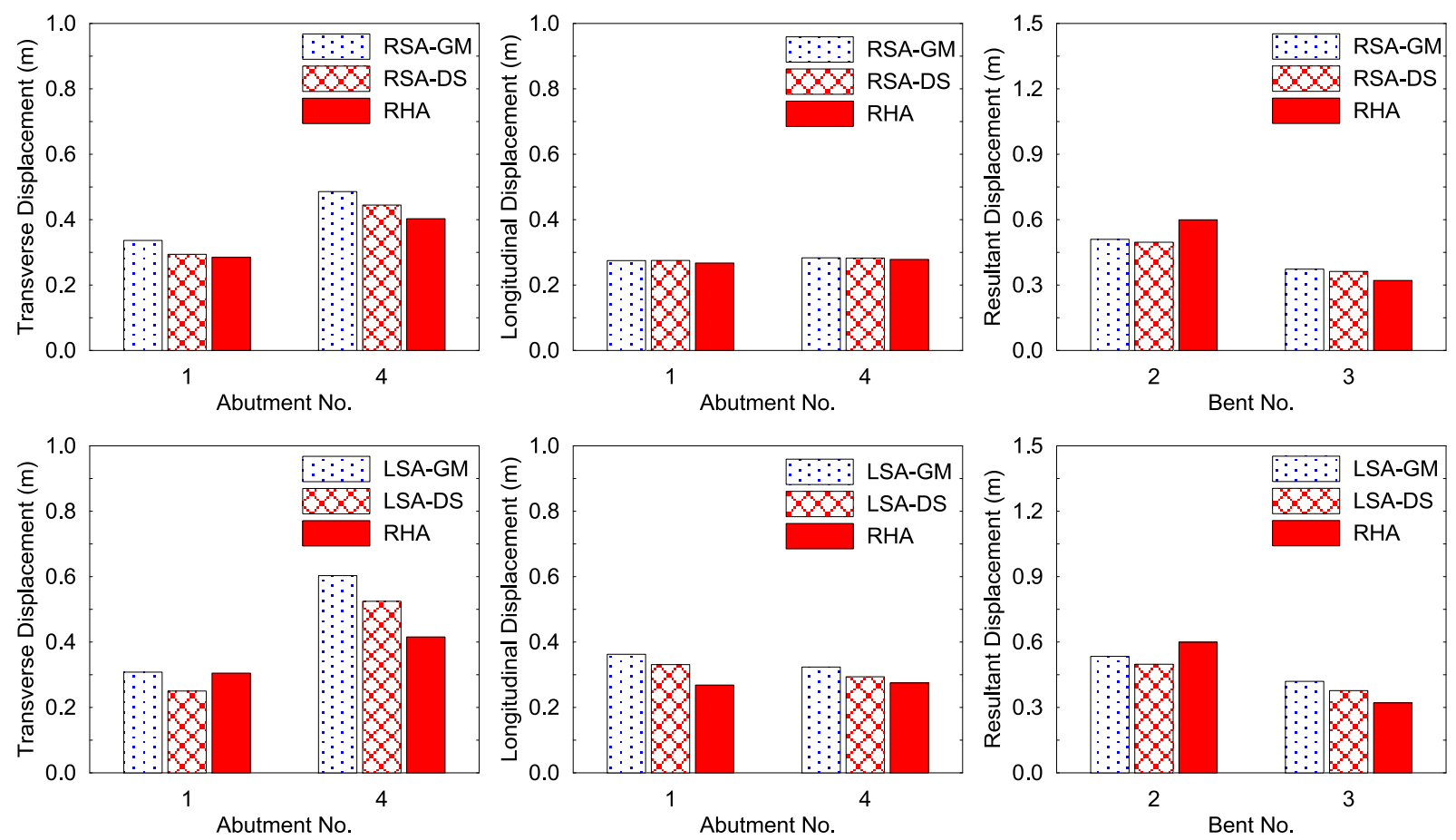

Figure C.23 - Results of Bridge 55-0837S when $\theta=21^{\circ}$ and longitudinal abutment stiffness $=0.55 K_{\text {eff }}$
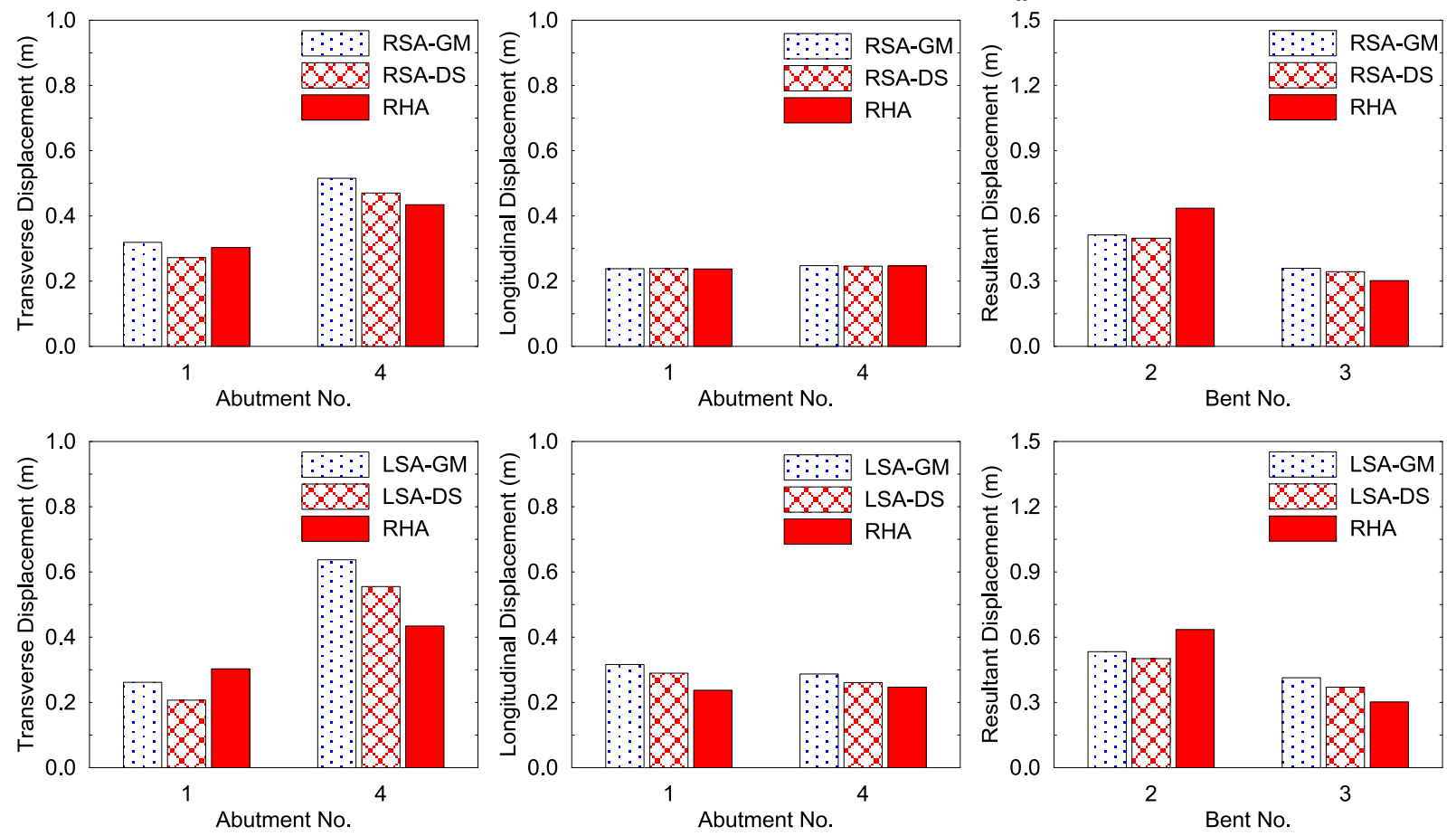

Figure C.24 - Results of Bridge 55-0837S when $\theta=21^{\circ}$ and longitudinal abutment stiffness $=1.00 K_{\text {eff }}$ 


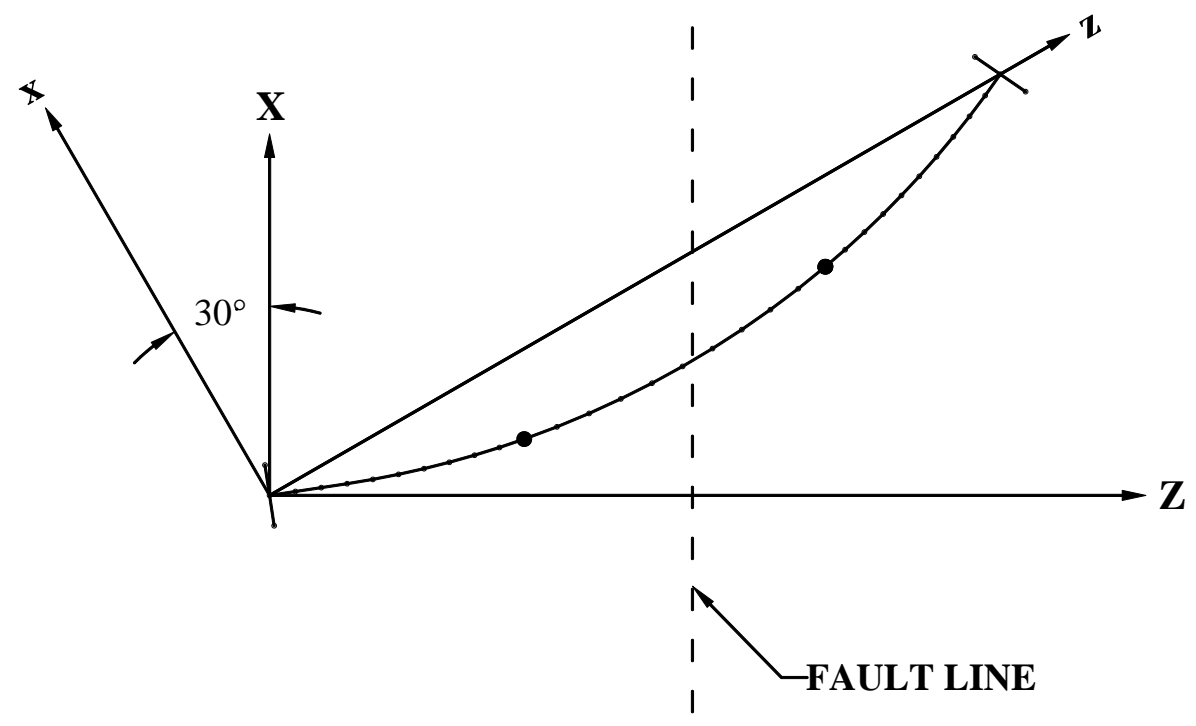

Figure C.25 - Sketch of Bridge 55-0837S when $\theta=30^{\circ}$
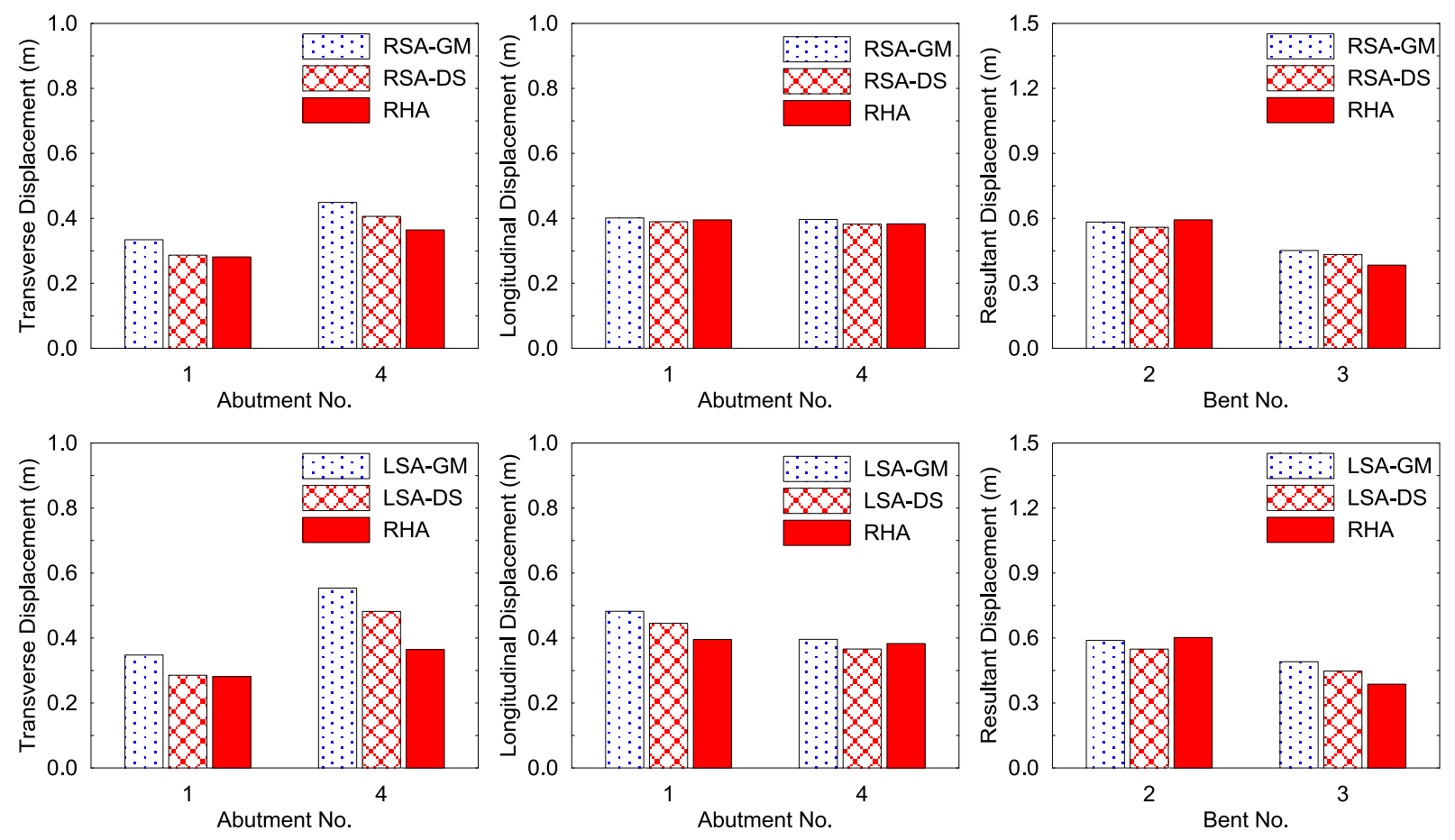

Figure C.26 - Results of Bridge 55-0837S when $\theta=30^{\circ}$ and longitudinal abutment stiffness $=0.10 K_{\text {eff }}$ 

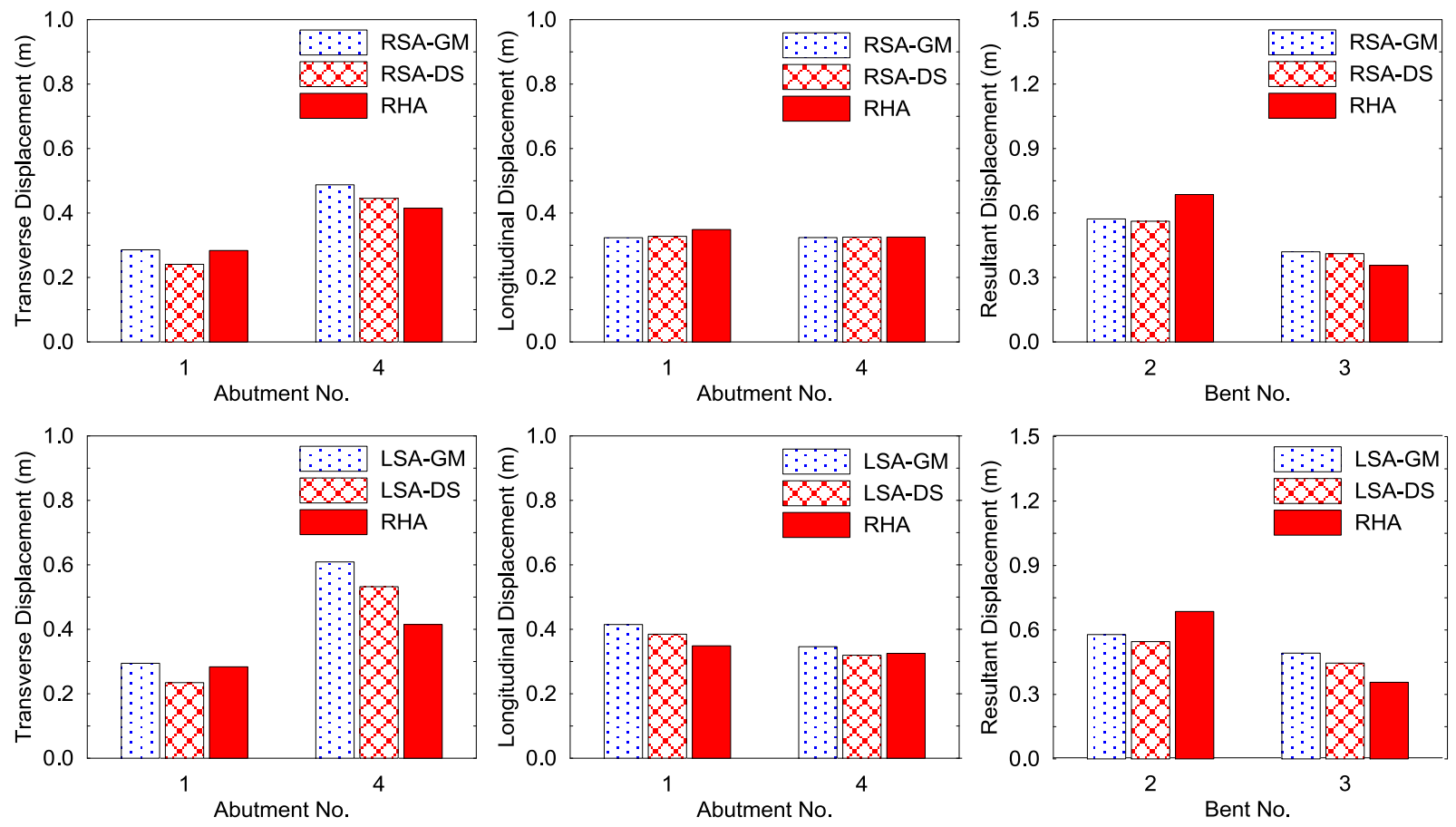

Figure C.27 - Results of Bridge 55-0837S when $\theta=30^{\circ}$ and longitudinal abutment stiffness $=0.55 K_{\text {eff }}$
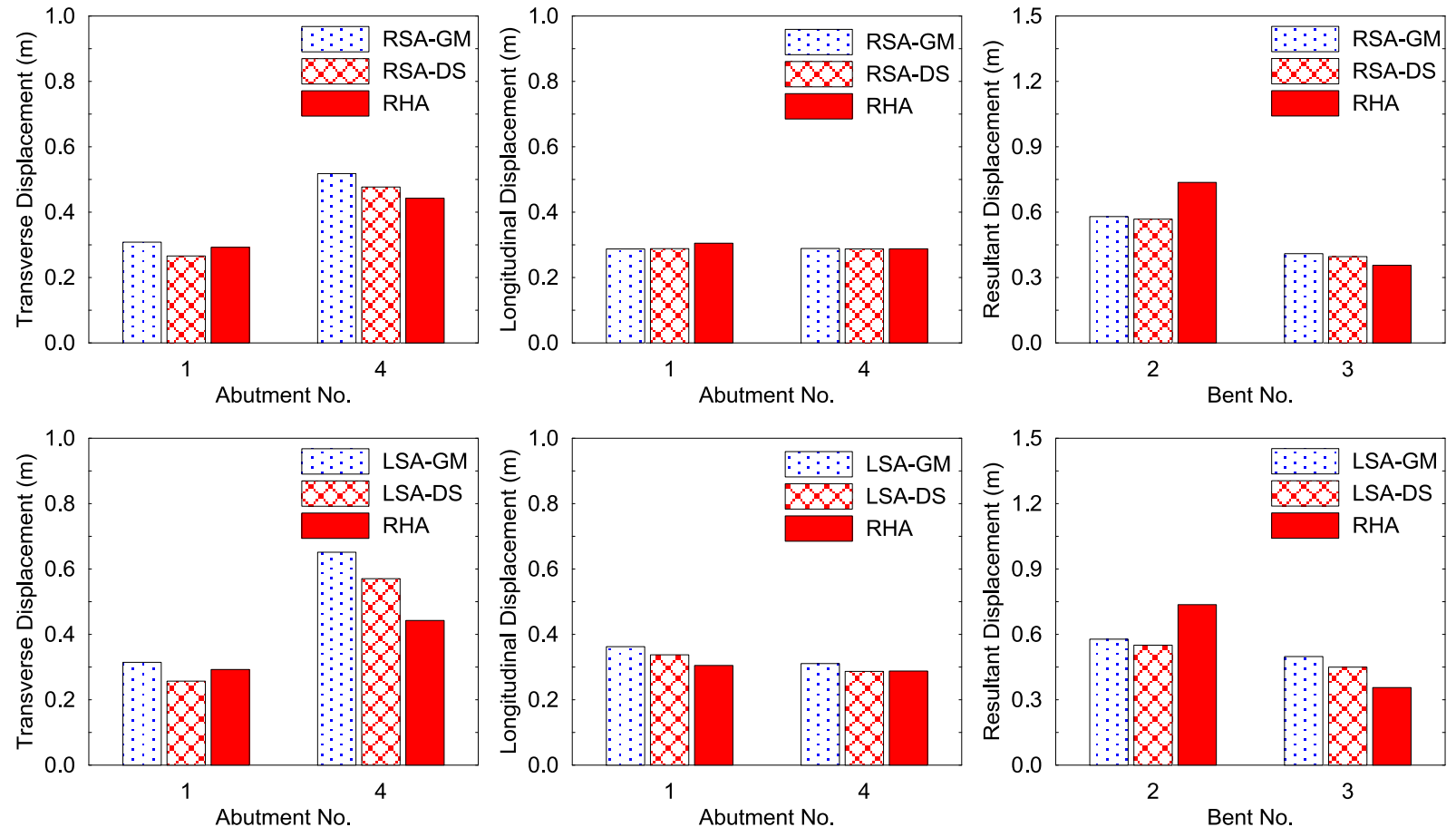

Figure C.28 - Results of Bridge 55-0837S when $\theta=30^{\circ}$ and longitudinal abutment stiffness $=1.00 K_{\text {eff }}$ 


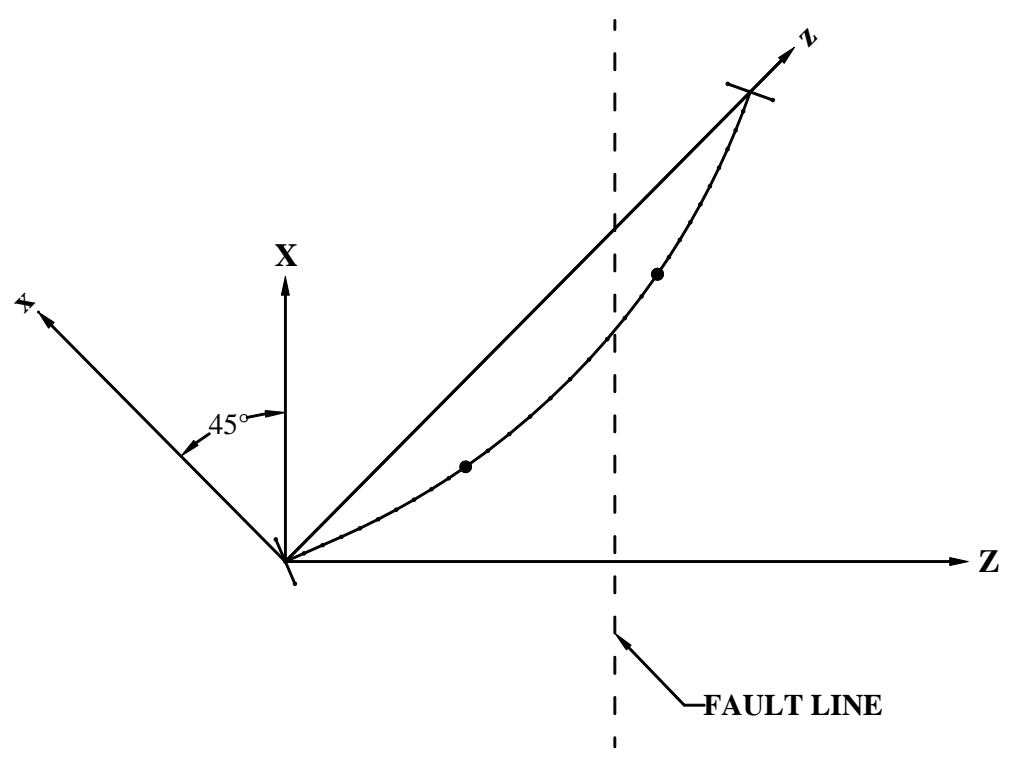

Figure C.29 - Sketch of Bridge 55-0837S when $\theta=45^{\circ}$
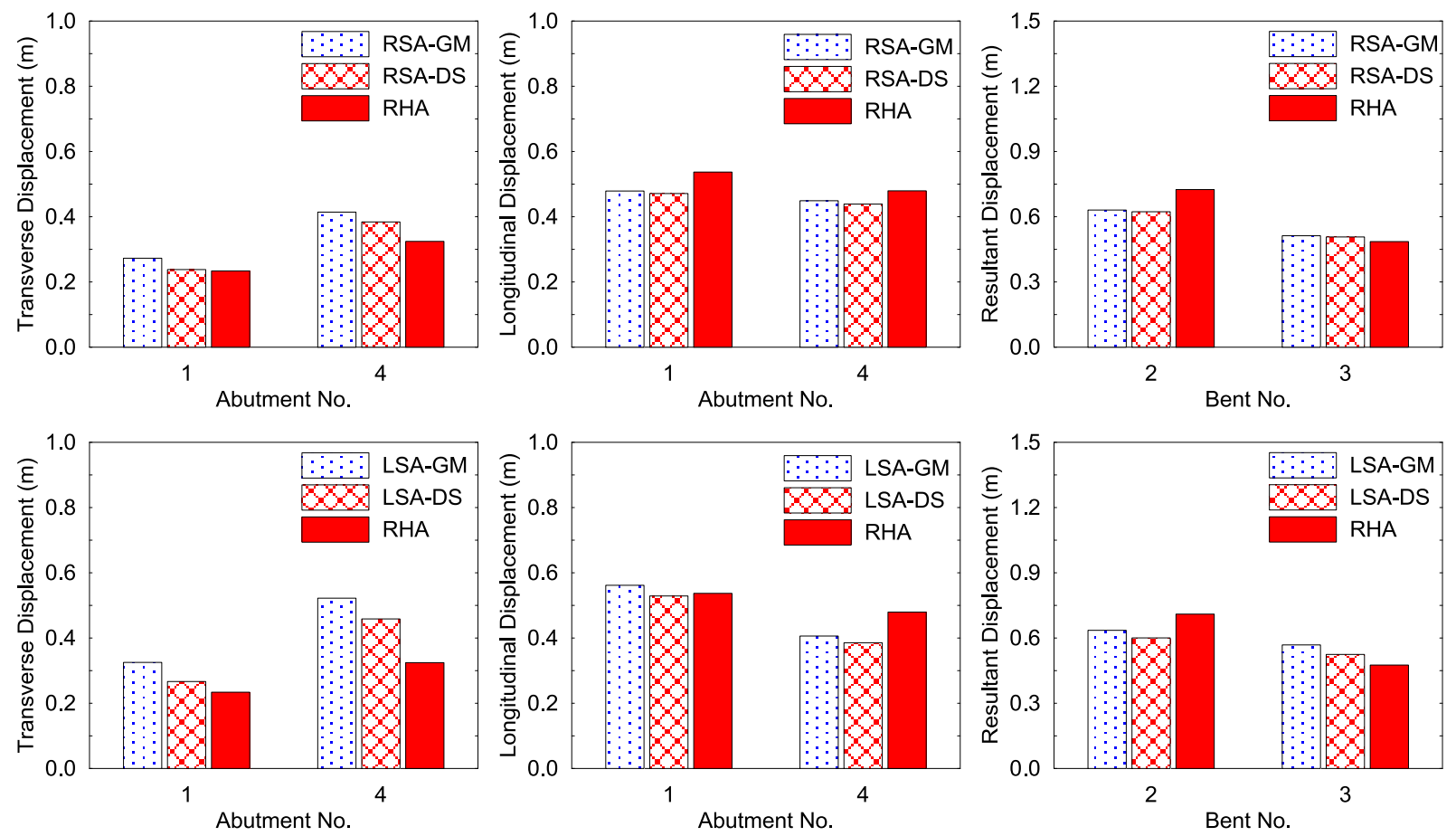

Figure C.30 - Results of Bridge 55-0837S when $\theta=30^{\circ}$ and longitudinal abutment stiffness $=0.10 K_{\text {eff }}$ 

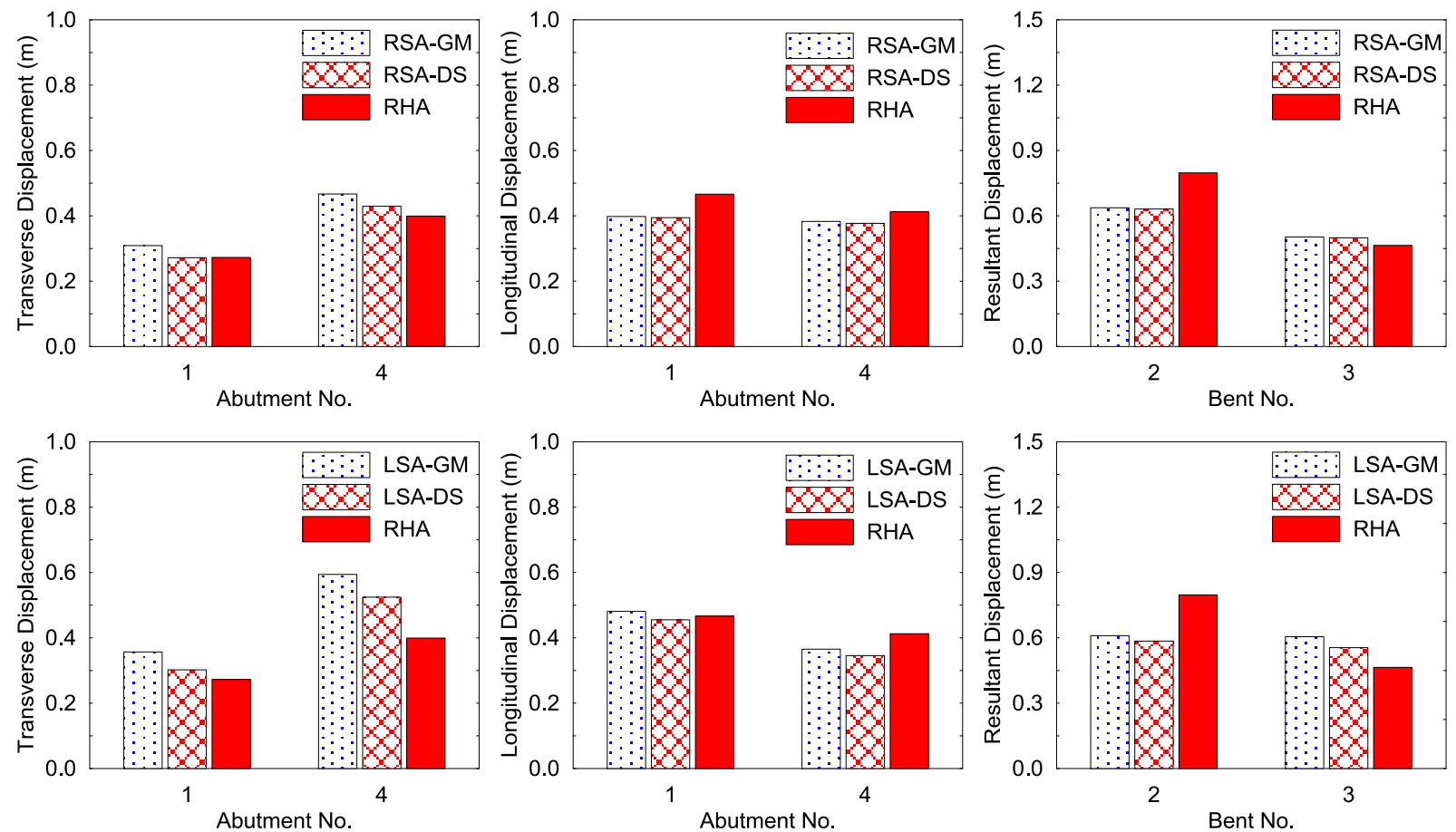

Figure C. 31 - Results of Bridge 55-0837S when $\theta=45^{\circ}$ and longitudinal abutment stiffness $=0.55 K_{\text {eff }}$
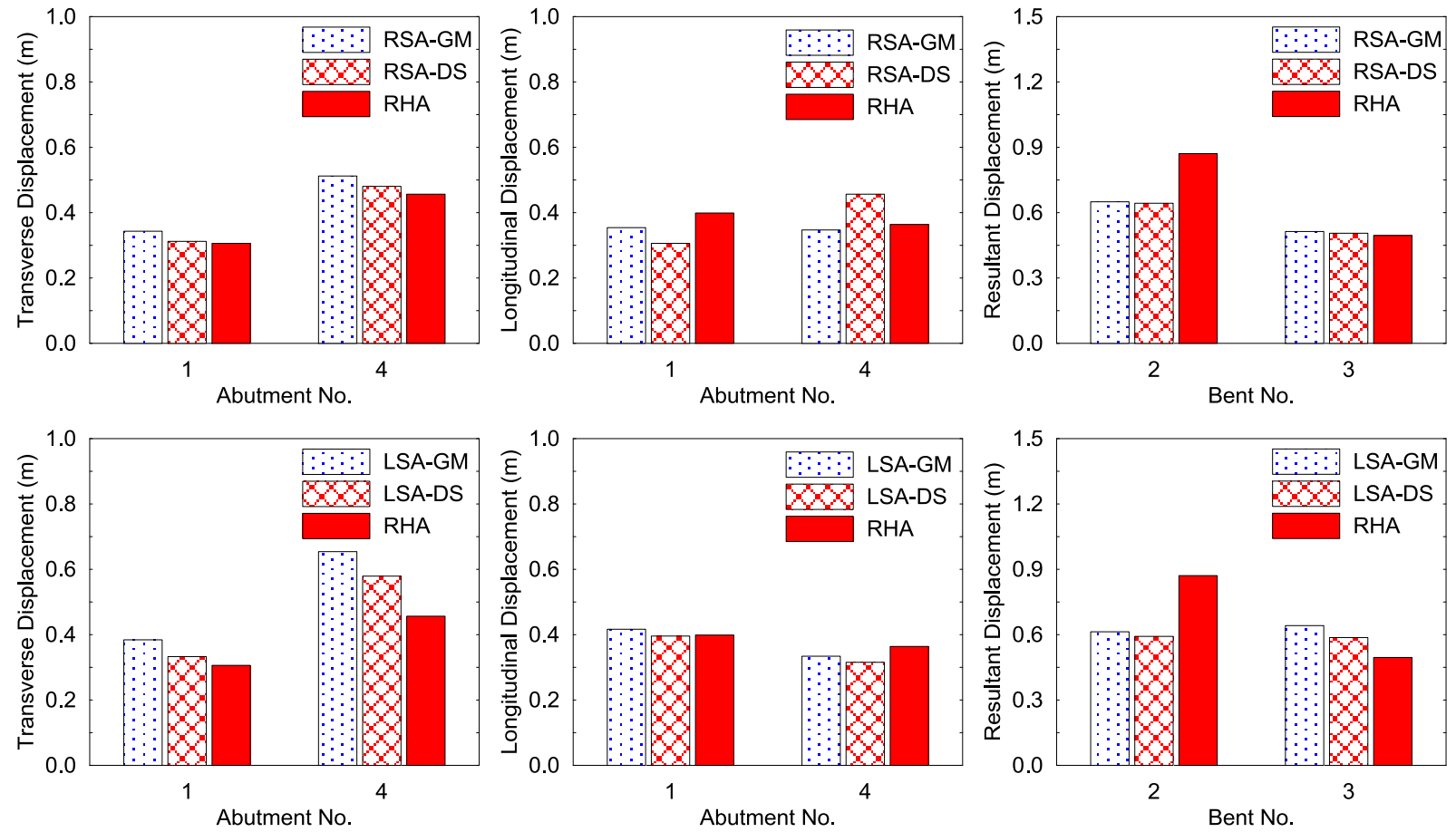

Figure C.32 - Results of Bridge 55-0837S when $\theta=45^{\circ}$ and longitudinal abutment stiffness $=1.00 K_{\text {eff }}$ 


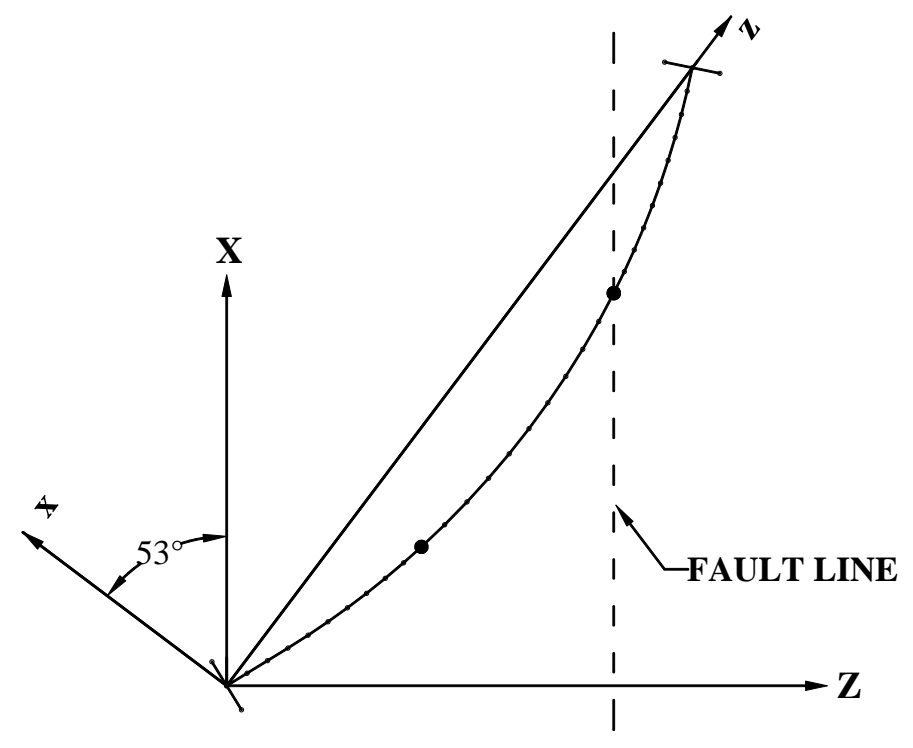

Figure C.33 - Sketch of Bridge 55-0837S when $\theta=53^{\circ}$
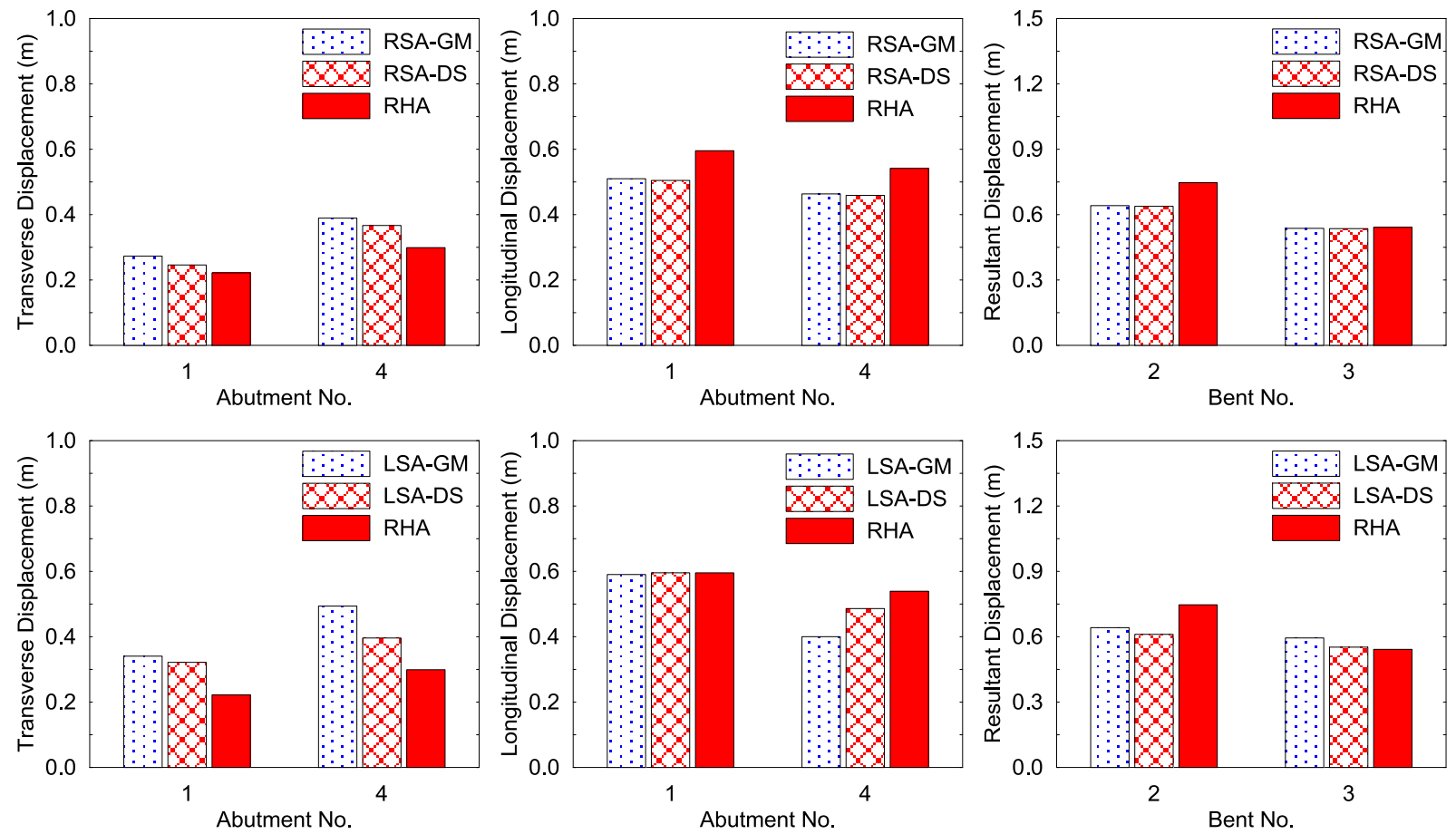

Figure C.34 - Results of Bridge 55-0837S when $\theta=53^{\circ}$ and longitudinal abutment stiffness $=0.10 K_{\text {eff }}$ 

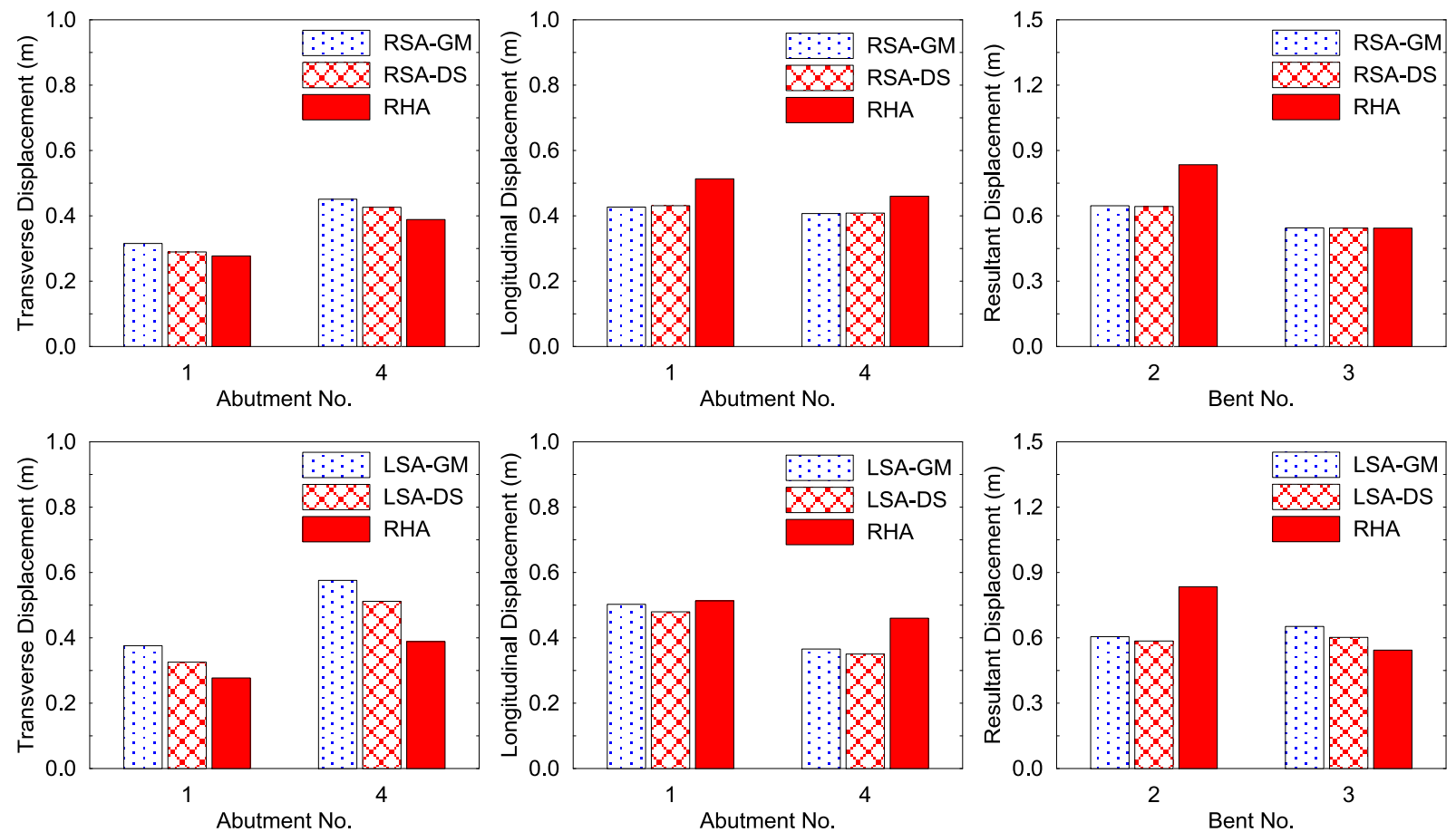

Figure C.35 - Results of Bridge 55-0837S when $\theta=53^{\circ}$ and longitudinal abutment stiffness $=0.55 K_{\text {eff }}$
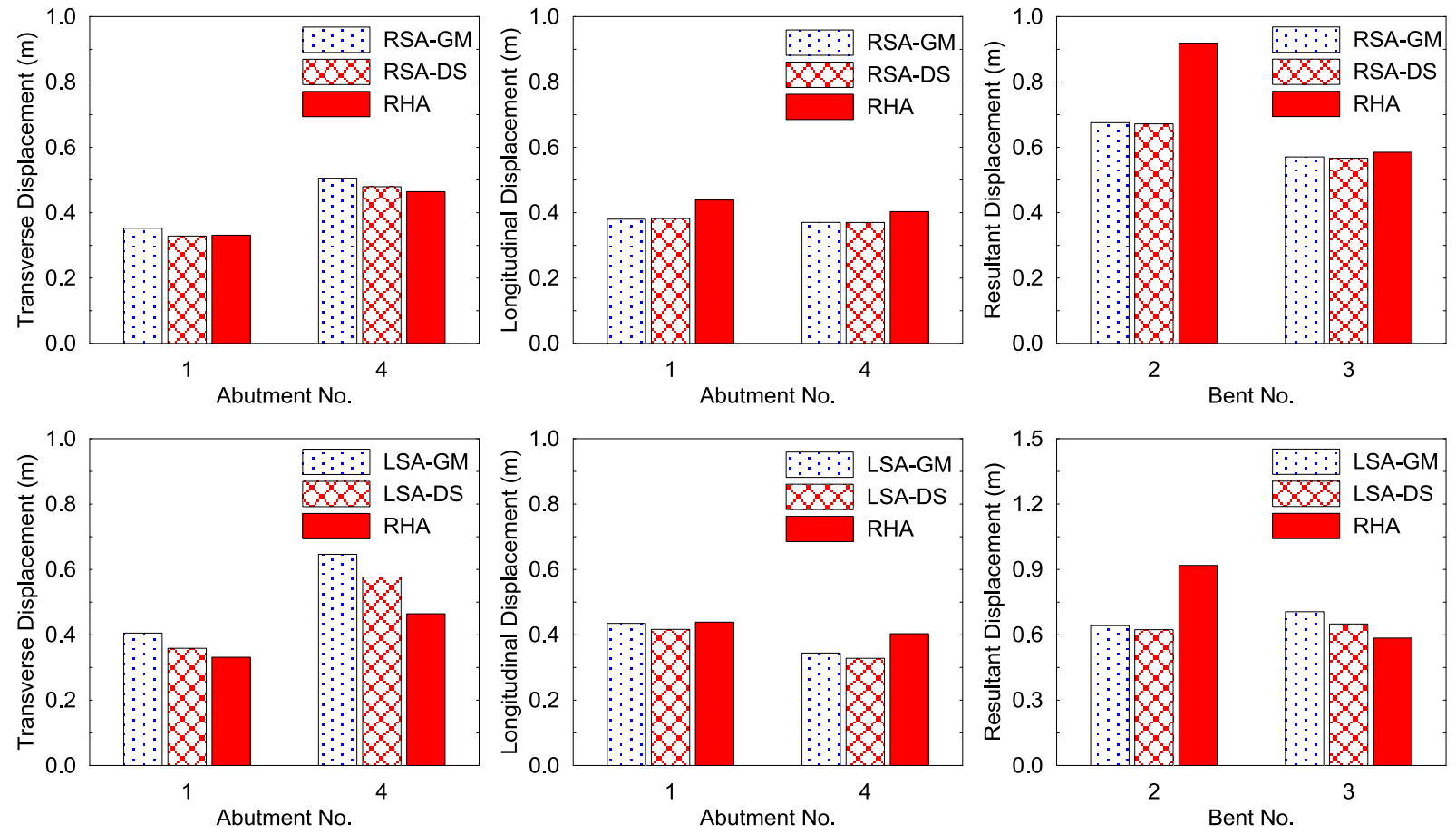

Figure C.36 - Results of Bridge 55-0837S when $\theta=53^{\circ}$ and longitudinal abutment stiffness $=1.00 K_{\text {eff }}$ 